\title{
Time Reversal of Acoustoelastic Lamb Waves
}

\section{Alan Conci Kubrusly}

Tese (Doutorado em Engenharia Mecânica). Pontifícia Universidade Católica do Rio de Janeiro. Rio de Janeiro, 2016. 


\section{Alan Conci Kubrusly}

\section{Time Reversal of Acoustoelastic Lamb Waves}

\section{TESE DE DOUTORADO}

DEPARTAMENTO DE ENGENHARIA MECÂNICA

Programa de Pós-Graduação em Engenharia

Mecânica 


\section{Alan Conci Kubrusly}

\section{Time Reversal of Acoustoelastic Lamb Waves}

Thesis presented to the Programa de Pós-Graduação em Engenharia Mecânica of the Departamento de Engenharia Mecânica, PUC-Rio as partial fulfillment of the requirements for the degree of Doutor em Engenharia Mecânica

Adviser : Prof. Arthur Martins Barbosa Braga

Co-Adviser: $\quad$ Prof. Jean Pierre von der Weid 


\section{Alan Conci Kubrusly}

\section{Time Reversal of Acoustoelastic Lamb Waves}

Thesis presented to the Postgraduate Program in Mechanical Engineering of the Departamento de Engenharia Mecânica, Centro Técnico Científico da PUC-Rio as partial fulfillment of the requirements for the degree of Doutor.

Prof. Arthur Martins Barbosa Braga

Advisor

Departamento de Engenharia Mecânica — PUC-Rio

Prof. Jean Pierre von der Weid

Co-Advisor

Centro de Estudos em Telecomunicações - PUC-Rio

Prof. Nicolás Leonardo Pérez Alvarez

Universidad de la Republica Uruguay

Prof. Ricardo Leiderman

Instituto de Computação - UFF

Prof. Miguel de Andrade Freitas

Centro de Estudos em Telecomunicações - PUC-Rio

Dr. Sergio Ricardo Kokay Morikawa

Petrobras

Prof. Márcio da Silveira Carvalho Coordinator of the Centro Técnico Científico - PUC-Rio 
All rights reserved.

\section{Alan Conci Kubrusly}

Alan Conci Kubrusly received a B.S degree in electronics and telecommunications engineering in 2009 and a M.S. degree in electrical engineering in 2012, both from the Pontifical Catholic University of Rio de Janeiro (PUC-Rio).

Bibliographic data

Kubrusly, Alan Conci

Time Reversal of Acoustoelastic Lamb Waves / Alan Conci Kubrusly; advisor: Arthur Martins Barbosa Braga; coadvisor: Jean Pierre von der Weid. - 2016.

176 f: il. color. ; $30 \mathrm{~cm}$

Tese (doutorado) - Pontifícia Universidade Católica do Rio de Janeiro, Departamento de Engenharia Mecânica, 2015.

Inclui bibliografia.

1. Engenharia Mecânica - Tese. 2. Ultrassom. 3. Ondas guiada. 4. Ondas de Lamb. 5. Acoustoelasticidade. 6. Inversão Temporal. 7. M.E.F. I. Braga, Arthur Martins Barbosa. II. von der Weid, Jean Pierre. III. Pontifícia Universidade Católica do Rio de Janeiro. Departamento de Engenharia Mecânica. IV. Título. 


\section{Acknowledgments}

To my advisers, Prof. Arthur Martins Barbosa Braga and Prof. Jean Pierre von der Weid, my deep and sincere gratitude for guidance, incentive and opportunity.

To CNPq for financial support.

To all members of the examination board for agreeing to participate, their time and effort in evaluating this thesis and valuable comments.

To Prof. Nicolás Leonardo Pérez Alvarez for continuous advices, priceless discussions and fruitful partnership.

To Prof. Julio Cezar Adamowski for his time and hospitality during the visits at the Ultrasound Laboratory of Polytechnic School of the University of São Paulo, and also for his suggestions.

To Prof. Marco Aurélio Brizzotti Andrade and Tiago dos Santos Ramos for their help with Ansys ${ }^{\circledR}$.

To Timóteo Francisco de Oliveira for manufacturing the piezocomposite transducers.

To Profs. Marcelo Roberto Baptista Pereira Luis Jimenez and Miguel de Andrade Freitas for their constant encouragement.

To colleagues Bruno César Cayres Andrade and Abel Arrieta Castro for their help to study for the qualify exam.

To Aura Conci for valuable discussions, Carlos Kubrusly for careful revision and Jessica Kubrusly for the help with the data.

To all the colleagues of CPTI for companionship.

To Chewbacca and Wolverine for faithful friendship and company during the writing of the thesis.

And foremost to my wife, Anna, my constant support, companion and love. Whose smile never let me give up. 


\section{Abstract}

Kubrusly, Alan Conci; Braga, Arthur Martins Barbosa; von der Weid, Jean Pierre. Time Reversal of Acoustoelastic Lamb Waves. Rio de Janeiro, 2016. 176p. PhD Thesis — Departamento de Engenharia Mecânica, Pontifícia Universidade Católica do Rio de Janeiro.

The acoustoelasticity studies the variation of the elastic waves velocity in bodies subject to an initial stress state. The acoustoelastic theory consists of non-linear relationship between stress and strain that rules the dynamic response superimposed to the initial pre-deformation. The acoustoelastic theory applied to guided waves is a very new branch of study. The theoretical development of this theory for Lamb waves was completed in 2012, which enabled the calculation of velocity variation as a function of stress. Lamb waves are elastic waves that propagate in plates obeying the boundary conditions on the surface. These waves are a priori dispersive. Finite Element Method (FEM) is an useful tool for ultrasonic waves propagation analysis. In order to address the acoustoelastic effect employing FEM one can use the effective elastic constants. By these effective constants it is possible to assemble an equivalent anisotropic stiffness tensor, which can be the material stiffness input in commercial software. The time reversal process is a well-known method for obtaining focused acoustic waves in both time and space. In the case of Lamb waves, the use of time-reversed signals compensates the dispersion of each propagation mode, recompressing the wave and producing a focused signal at the reception position. In this thesis the new branch of acoustoelasticity for Lamb waves is thoroughly analyzed and its state of the art is reviewed. The possibility of using effective elastic constants for determination of the velocity dependence on stress and the errors in this approximation are investigated. The use of FEM in Ansys ${ }^{\circledR}$ is validated for single mode under uniaxial tensile stress. Then, the simulations of wideband multi-mode acoustoelastic Lamb waves is carried out in the numerical software, the time-reversal focusing ability is verified and the effect of uniaxial load on the focus is investigated. Finally, experiments were performed in an aluminum plate longitudinally loaded. The feasibility of using the time reversal focus characteristics in order to measure the strain is concluded and its limitations are pointed out.

\section{Keywords}

Ultrasonic; Guided Waves; Lamb waves; Acoustoelasticity; TimeReversal; F.E.M.; 


\section{Resumo}

Kubrusly, Alan Conci; Braga, Arthur Martins Barbosa; von der Weid, Jean Pierre. Inversão temporal de ondas de Lamb acoustoelásticas. Rio de Janeiro, 2016. 176p. Tese de Doutorado - Departamento de Engenharia Mecânica, Pontifícia Universidade Católica do Rio de Janeiro.

A acoustoelasticidade estuda a variação da velocidade de ondas elásticas em corpos sujeitos a um estado de tensão inicial. A teoria da acoustoelasticidade consiste-se de um relação não-linear entre tensão e deformação que rege a resposta dinâmica de uma onda elástica sobreposta à pré deformação inicial. A teoria da acoustoelasticidade aplicada a ondas guiadas é um ramo de estudo extremamente novo. Seu desenvolvimento teórico para ondas de Lamb foi concluída em 2012, o que permitiu o cálculo da variação da velocidade em função da tensão. Ondas de Lamb são ondas elástica que propagam-se em placas de faces paralelas obedecendo às condições de contorno nas superfícies. Essas ondas são a princípio dispersivas. Modelagem por elementos finitos (MEF) é uma ferramenta útil para a análise das ondas ultrassônicas. A fim de abordar o efeito acoustoelástico utilizando método de elementos finitos pode-se utilizar as constantes elásticas eficazes. Por meio dessas é possível montar um tensor de rigidez anisotrópico equivalente, que por sua vez pode ser utilizado como a entrada de rigidez do material em softwares comerciais. O processo de inversão temporal é um método bem conhecido para a obtenção de ondas acústicas focalizadas no tempo e no espaço. No caso de ondas de Lamb, o uso de sinais de inversão temporal compensa a dispersão de cada modo de propagação, recomprime e focaliza a onda na posição de leitura. Nesta tese, o novo ramo da acoustoelasticidade em ondas de Lamb foi analisado e seu estado da arte revisado. A possibilidade de utilizar constantes elásticas eficazes para determinação da dependência da velocidade com a tensão e os erros nesta aproximação foram investigados. O uso do programa de elementos finitos Ansys ${ }^{\circledR}$ foi validado para propagação de ondas de Lamb monomodo em uma placa submetida a tração uniaxial. Em seguida, simulações de propagação de ondas de Lamb multimodais acoustoelásticas foram realizadas através do software e o efeito da carregamento na focalização foi analisado numericamente. Finalmente, experimentos foram realizadas em uma placa de alumínio carregada longitudinalmente. A viabilidade e limitações da utilização das características de focalização por inversão temporal a fim de medir a tensão são apontadas.

\section{Palavras-chave}

Ultrassom; Ondas guiada; Ondas de Lamb; Acoustoelasticidade; Inversão Temporal; M.E.F; 


\section{Contents}

$\begin{array}{ll}\text { Summary of notations } & 13\end{array}$

1 Introduction $\quad 15$

2 Guided waves in plates $\quad \mathbf{2 0}$

2.1 Wave equation for elastic media 20

2.2 Lamb waves 22

2.3 Signal processing for guided waves analysis 25

2.3.1 Extracting dispersion from sensed signal 25

2.3.1.1 Two-dimension Fourier transform 26

$\begin{array}{lll}\text { 2.3.1.2 Phase spectral analysis } & 27\end{array}$

2.3.2 Dispersion compensation and mode separation 28

3 Acoustoelastic equations of motion 30

3.1 Finite deformation elasticity 31

3.1.1 Equilibrium condition and motion equation 34

3.1.2 Incremental constitutive and wave equations 36

3.2 Particular case of null rotational on strain 40

3.3 Tensor symmetries 41

3.3.1 Numerical example 43

3.4 Relationship between velocity at initial and natural frames for bulk waves 45

$4 \quad$ Acoustoelastic Lamb waves $\quad 46$

4.1 Partial wave technique for acoustoelastic Lamb wave propagation 47

4.1.1 Note on symmetry assumptions $\quad 52$

4.2 Computational routine 53

4.3 Particular case of longitudinal applied load in naturally isotropic medium 54

4.4 Partial wave technique for anisotropic medium 56

4.4.1 Approximation for acoustoelastic case using Effective Elastic Constants 57

5 Acoustoelastic Lamb waves dispersion curves $\quad 60$

5.1 Natural reference frame 60

$\begin{array}{lll}5.1 .1 & \text { A0 mode } & 61\end{array}$

5.1.2 S0 mode 66

$\begin{array}{ll}\text { 5.1.3 Higher order modes } & 68\end{array}$

$\begin{array}{lll}5.2 & \text { Initial reference frame } & 69\end{array}$

5.3 Relationship between velocity at initial and natural frames 71

5.4 Effective Elastic Constants approximation 74

5.4.1 Approximation at natural reference frame $\quad 74$

$\begin{array}{ll}\text { 5.4.2 Approximation at initial reference frame } & 79\end{array}$

5.5 Review of literature results $\quad 80$

6 Lamb waves simulation $\quad 83$

$\begin{array}{ll}6.1 \text { Validation for unstressed medium } & 87\end{array}$

$\begin{array}{lll}6.1 .1 & \text { A0 mode } & 87\end{array}$ 
$\begin{array}{ll}\text { 6.1.2 S0 mode } & 91\end{array}$

6.2 Simulation for natural reference frame $\quad 94$

$\begin{array}{ll}\text { 6.3 Simulation for initial reference frame } & 97\end{array}$

$7 \quad$ Time reversal of acoustic waves $\quad 99$

$\begin{array}{lll}7.1 & \text { Time reversal in plates } & 100\end{array}$

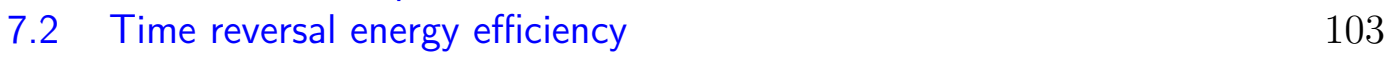

$\begin{array}{lll}7.3 & \text { Time reversal spatial focusing } & 105\end{array}$

$\begin{array}{lll}\text { 7.4 Effect of longitudinal strain on time reversal signal } & 105\end{array}$

$\begin{array}{lll}7.5 & \text { One-bit time reversal in plates } & 108\end{array}$

8 Simulation of wideband time reversal of Lamb waves under longitudinal stress $\quad \mathbf{1 1 0}$

8.1 Infinitely long plate 111

8.1.1 Effect of longitudinal stress on time reversal 114

$\begin{array}{ll}\text { 8.1.1.1 Analytical model } & 116\end{array}$

$\begin{array}{ll}\text { 8.1.1.2 Influence of sensing position } & 117\end{array}$

8.1.1.3 Band limited propagation 119

8.1.1.4 One-Bit Time Reversal 122

$\begin{array}{ll}\text { 8.2 Finite length plate } & 124\end{array}$

$\begin{array}{ll}\text { 8.2.1 Effect of longitudinal stress on time reversal } & 127\end{array}$

$\begin{array}{ll}\text { 8.2.1.1 Influence of sensing position } & 128\end{array}$

8.2.1.2 Band limited propagation 130

8.2.1.3 One-bit time reversal 132

9 Experiments $\quad 134$

$\begin{array}{lll}9.1 & \text { Piezocomposite transducers } & 135\end{array}$

$\begin{array}{ll}\text { 9.2 Time reversal in plates } & 136\end{array}$

$\begin{array}{ll}9.2 .1 \text { Strain sensing } & 138\end{array}$

9.2.2 Transducer combination 140

9.3 Strain sensing by one-bit time reversal in plates 141

9.4 Strain sensing by correlation implementation of time reversal in plates 143

9.5 Comparison between experiments and simulation 145

10 Conclusion $\quad 148$

10.1 Future works 150

A Verification of stiffness and acoustoelastic tensors symmetries 152

A.1 Stiffness tensors 152

A.1.1 Second order stiffness tensor symmetries 152

A.1.2 Third order stiffness tensor symmetries 153

$\begin{array}{lll}\text { A.2 Symmetries on acoustoelastic tensors } & 155\end{array}$

$\begin{array}{lll}\text { A.2.1 Acoustoelastic tensors for naturally isotropic medium } & 157\end{array}$

$\begin{array}{lll}\text { B Publications } & 159\end{array}$

$\begin{array}{lr}\text { Bibliography } & 160\end{array}$ 


\section{List of Figures}

2.1 Plate geometry and Lamb waves. 23

2.2 Modes shape. 23

2.3 Dispersion curves for an aluminum plate. 25

3.1 Three states configuration. 32

5.1 Acoustoelastic phase velocity dispersion curves for $A 0$ mode at natural frame of reference.

5.2 Acoustoelastic group velocity dispersion curves for $A 0$ mode at natural frame of reference.

5.3 Acoustoelastic velocity sensitivity for $A 0$ mode at natural frame of reference.

5.4 Acoustoelastic phase velocity dispersion curves for $S 0$ mode at natural frame of reference.

5.5 Acoustoelastic group velocity dispersion curves for $S 0$ mode at natural frame of reference.

5.6 Acoustoelastic velocity sensitivity for $S 0$ mode at natural frame of reference.

5.7 Acoustoelastic phase and group velocity sensitivity for up to second order modes at natural frame of reference.

5.8 Acoustoelastic phase velocity dispersion curves for $A 0$ mode at initial frame of reference.

5.9 Acoustoelastic group velocity dispersion curves for $A 0$ mode at initial frame of reference.

5.10 Acoustoelastic phase and group velocity sensitivity for up to second order modes at initial frame of reference.

5.11 Acoustoelastic phase and group velocity dispersion curves for $S 0$ mode at initial frame of reference.

5.12 Acoustoelastic velocity difference from initial to natural frames for A0.

5.13 Acoustoelastic velocity difference from initial to natural frames for S0.

5.14 Acoustoelastic sensitivity difference from initial to natural frames for $A 0$.

5.15 Acoustoelastic sensitivity difference from initial to natural frames for $S 0$.

5.16 Acoustoelastic phase velocity dispersion curves for $A 0$ mode at natural frame of reference, EEC1 approximation.

5.17 Acoustoelastic group velocity dispersion curves for $A 0$ mode at natural frame of reference, EEC1 approximation.

5.18 Acoustoelastic phase and group velocity dispersion curves for $S 0$ mode at natural frame of reference, EEC1 approximation.

5.19 EEC velocity error for $A 0$ mode at natural frame of reference. $\quad 77$

5.20 EEC velocity error for $S 0$ mode at natural frame of reference. $\quad 78$

5.21 Sensitivity error for EEC at natural frame of reference. 
5.22 Sensitivity error for EEC at initial frame of reference.

6.1 Modeled plate.

6.2 MLK-NRB absorber. $\quad 86$

6.3 A0 excitation signal. $\quad 88$

6.4 A0 propagation. $\quad 89$

6.5 A0 2DFT.

6.6 A0 dispersion extraction. 90

6.7 S0 excitation signal. $\quad 91$

$\begin{array}{ll}6.8 \text { SO FEM propagation. } & 92\end{array}$

6.9 S0 2DFT.

6.10 S0 dispersion extraction. $\quad 94$

6.11 A0 FEM response for stressed medium. $\quad 95$

6.12 A0 dispersion extraction for stressed medium. $\quad 96$

6.13 S0 dispersion extraction for stressed medium. $\quad 97$

7.1 Time reversal process, steps 1 and $2 . \quad 101$

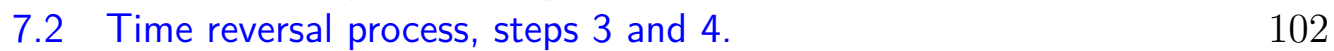

7.3 Example signal for time reversal energy efficiency. 104

$\begin{array}{lll}7.4 & \text { Example of typical spatial focus pattern. } & 105\end{array}$

7.5 Time reversal process under longitudinal strain, steps 3 and $4 . \quad 106$

7.6 Ideal variations of the time reversal focus under strain. 108

8.1 Wideband excitation signal. 110

8.2 Dispersion curves for a $3 \mathrm{~mm}$ thick aluminum plate. 111

8.3 Wideband Lamb waves propagation. 112

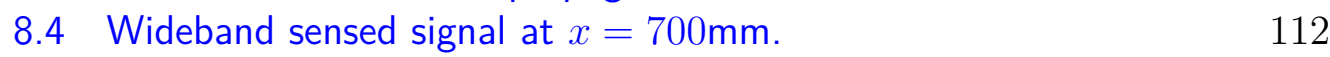

8.5 Dispersion compensation. 113

8.6 Wideband time reversal Lamb waves propagation. 113

8.7 Wideband time reversal signal sensed at $x=700 \mathrm{~mm}$. 114

8.8 Wideband time reversal focus sensed at $x=700 \mathrm{~mm}$ for several longitudinal stress levels. $\quad 115$

8.9 Time reversal focus behavior under stress at $x=700 \mathrm{~mm}$. $\quad 115$

8.10 Wideband time reversal focus at $x=700 \mathrm{~mm}$ for several longitudinal stress levels calculated from analytical model.

8.11 Wideband Lamb waves time reversal for several sensed position propagation.

8.12 Wideband time reversal sensitivity of Lamb waves for several sensed position.

8.13 Acoustoelastic phase and group velocity sensitivity at natural frame of reference for a $3 \mathrm{~mm}$ thick plate.

8.14 Filtered signals.

8.15 Filtered time reversal signals. 121

8.16 Time reversal focus behavior for filtered signals. 122

8.17 Wideband one-bit time-reversed signal. 123

8.18 Wideband one-Bit time reversal signal. 123

8.19 Filtered one-bit time reversal signals. 124

8.20 One-bit time reversal focus behavior for filtered signals. 124

8.21 Wideband Lamb waves propagation in finite plate. 125 
8.22 Wideband sensed signal at $x=700 \mathrm{~mm}$ for finite length plate.

8.23 Wideband time reversal Lamb waves propagation for finite length plate.

8.24 Wideband time reversal signal sensed at $x=700 \mathrm{~mm}$ for finite length plate.

8.25 Wideband time reversal focus sensed at $x=700 \mathrm{~mm}$ for several longitudinal stress levels in a finite plate.

8.26 Time reversal focus behavior under stress at $x=700 \mathrm{~mm}$ in a finite plate.

8.27 Wideband Lamb waves time reversal for several sensed position propagation in finite plate.

8.28 Wideband Lamb waves time reversal sensitivity for several sensed position propagation in finite plate.

8.29 Filtered signals for finite plate.

8.30 Filtered time-reversal signals for finite length plate.

8.31 Time reversal focus behavior for filtered signals in finite length plate. 132

8.32 Wideband One-Bit Time Reversal signal for finite length plate. 132

8.33 Filtered one-bit time reversal signals for finite length plate.

8.34 One-bit time reversal focus behavior for filtered signals in finite length plate.

9.1 Experimental setup.

9.2 Received forward signal at each transducer. 137

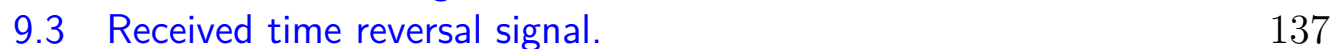

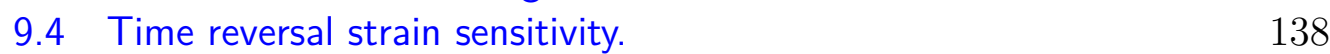

9.5 Received forward signal for transducer combination. $\quad 140$

9.6 Received time reversal signal for transducer combination. 141

9.7 Received one-bit time reversal signal. 142

9.8 One-bit time reversal strain sensitivity. 143

9.9 Correlation implementation of time reversal signal. 144

9.10 Correlation implementation of one-bit time reversal signal. 145 


\section{List of Tables}

3.1 Extended Voigt notation. 43

3.2 Aluminum constants. 43

$\begin{array}{ll}4.1 & \text { Voigt notation. } \\ \end{array}$

5.1 Stress and strain states used at calculations. $\quad 60$

6.1 Ansys notation for stiffness matrix. 84

6.2 FEM A0 results. $\quad 91$

6.3 FEM S0 results. $\quad 94$

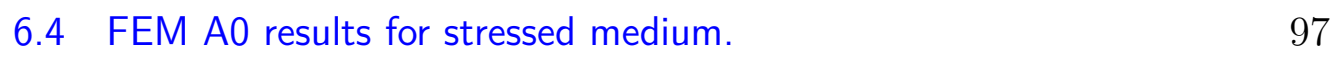

6.5 FEM S0 results for stressed medium. $\quad 97$

6.6 FEM A0 results for stressed medium at initial frame of reference. 98

6.7 FEM S0 results for stressed medium at initial frame of reference. 98

8.1 Wideband time reversal focus characteristics. 114

8.2 Filter parameters. 119

8.3 Filtered time reversal focus and sensitivity characteristics, conventional and one-bit. 122

8.4 Wideband time reversal focus characteristics for finite length plate. 126

8.5 Filtered time reversal focus and sensitivity characteristics, conventional and one-bit, for finite length plate. 131

9.1 Piezocomposites constructive characteristics. 135

9.2 Sensitivity Results. 139

9.3 Sensitivity Results for One-bit time reversal. 142

9.4 Sensitivity results for correlation implementation. 144 


\section{Summary of notations}

$\boldsymbol{\sigma}$ Stress tensor. Unity: $\mathrm{Pa}$

$\rho$ Mass density. Unity: $\mathrm{kg} / \mathrm{m}^{3}$

$\boldsymbol{u}$ Displacement vector. Unity: $\mathrm{m}$

t. Time. Unity: s

$\varepsilon$ Strain tensor. Unity: Dimensionless

$\boldsymbol{C}$ or $C_{i j k l}$ Fourth order stiffness tensor. Unity: $\mathrm{Pa}$

$\delta_{i j}$ Kroenecker delta tensor.

$I_{i j k l}$ Auxiliary tensor expressed as a function of the Kroenecker tensor.

$\omega$. Angular frequency. Unity: $\mathrm{rad} / \mathrm{s}$

$f$ Ordinary frequency. Unity: $\mathrm{Hz}$

$\boldsymbol{\kappa}$ Wave vector. Unity: $1 / \mathrm{m}$

$\lambda$ First Lamé constant. Unity: Pa

$\mu$ Second Lamé constant. Unity: Pa

$c_{L}$ Longitudinal bulk wave velocity. Unity: $\mathrm{m} / \mathrm{s}$

$c_{T}$ Transverse bulk wave velocity. Unity: $\mathrm{m} / \mathrm{s}$

$\kappa$ wavenumber. Unity: $1 / \mathrm{m}$

$d$ Plate thickness. Unity: $\mathrm{m}$

$h$ Plate half-thickness. Unity: $\mathrm{m}$

$c_{g r}$ Group velocity. Unity: $\mathrm{m} / \mathrm{s}$

$c_{p h}$ or $c$ Phase velocity. Unity: $\mathrm{m} / \mathrm{s}$

$\ell$ Propagation distance. Unity: $\mathrm{m}$

$(\hat{\bullet})$ Fourier transform of $(\cdot)$.

$|(\cdot)|$ Modulus of $(\cdot)$.

$\boldsymbol{\xi}$ or $\xi_{\alpha}$ coordinate in the natural state of reference. Unity: $\mathrm{m}$

$\boldsymbol{X}$ or $X_{I}$ coordinate in the initial state of reference. Unity: $\mathrm{m}$

$\boldsymbol{x}^{\prime}$ or $x_{i}^{\prime}$ coordinate in the final state of reference. Unity: $\mathrm{m}$

$\boldsymbol{x}$ or $x_{i}$ coordinate in unspecified state of reference. Unity: $\mathrm{m}$

$(\cdot)^{n}$ Entity referenced to the natural state of reference.

$(\cdot)^{i}$ Entity referenced to the initial state of reference.

$(\cdot)^{f}$ Entity referenced to the final state of reference.

$\boldsymbol{C}$ or $C_{i j k l m n}$ Sixth order stiffness tensor. Unity: $\mathrm{Pa}$

$l, m$ and $n$ Murnaghan Constants. Unity: Pa

$W$ strain energy function. Unity: $\mathrm{J} / \mathrm{m}^{3}$ or $\mathrm{Pa}$ 
$s$ Second Piola-Kirchhoff stress tensor. Unity: Pa

$\boldsymbol{t}$ Cauchy stress tensor. Unity: Pa

$\boldsymbol{T}$ Incremental stress tensor. Unity: $\mathrm{Pa}$

$\boldsymbol{F}$ Gradient of Deformation. Unity: Dimensionless

$\boldsymbol{E}$ Lagrangian strain tensor. Unity: Dimensionless

$\boldsymbol{B}$ Acoustoelastic incremental constitutive tensor. Unity: Pa

$\boldsymbol{A}$ Acoustoelastic tensor. Unity: Pa

$\boldsymbol{K}$ Christoffel equation in matricial form. Unity: $\mathrm{Pa}$

$s^{\sigma}$ Velocity sensitivity to stress. Unity: $1 / \mathrm{Pa}$

$s^{\varepsilon}$ Velocity sensitivity to strain. Unity: Dimensionless

$E$ Young modulus. Unity: Pa

$\lambda$ Wavelength. Unity: $\mathrm{m}$

FEM Finite element method.

$l_{e}$ Finite element length: Unity: $\mathrm{m}$

$f_{s}$ Sampling frequency. Unity: $\mathrm{Hz}$

$\Delta T$ Duration of reception time window. Unity: s

$t_{0}$ Reception initial time. Unity: $\mathrm{s}$

$T_{e}$ Transmitter transducer.

$T_{r}$ Receiver transducer.

$H$ System transfer function.

$x_{0}$ Initial transmitted signal.

$y_{0}$ Received signal.

$x_{1}$ Time-reversed transmitted signal.

$y_{1}$ Time-reversal received signal.

* Convolution.

TREF Time-reversal energy efficiency. Unity: Dimensionless

$E_{p k}$ Time-reversal signal energy inside the focused interval. Unity: $\mathrm{V}^{2} \mathrm{~S}$

$E_{\text {tot }}$ Total signal energy. Unity: $\mathrm{V}^{2} \mathrm{~S}$

$\operatorname{sign}\{(\cdot)\}$ Sign of $(\cdot)$.

$x_{1 b i t}$ One-bit time-reversed transmitted signal.

$y_{1 b i t}$ One-bit time-reversal received signal.

$L$ Plate length. Unity: $\mathrm{m}$

$W$ Plate width. Unity: $\mathrm{m}$

TOF Time-of-flight. Unity: s

SNR Signal-to-noise ratio. Unity: Dimensionless

$R^{2}$ Coefficient of Determination. Unity: Dimensionless

$\mathcal{I}_{i j k l m n}$ Auxiliary tensor expressed as a function of the Kroenecker tensor.

$\mathbb{I}_{i j k l m n}$ Auxiliary tensor expressed as a function of the Kroenecker tensor. 


\section{1 \\ Introduction}

Monitoring and measuring the state of deformation of a mechanical structure is an important branch of study in mechanical engineering. The introduction of sensor networks to monitor the mechanical strain and stress state of structures as subsea risers [1-5], wellbores [6-8], airplane wings [9-11], bridges [12] and metal cranes [13], are becoming a common practice reducing risk and saving money.

Ultrasonic methods [14] are widely used in nondestructive evaluation (NDE), specially aiming to identify some kind of damage [15]. It offers advantages as low-cost, robustness and easiness to install, allowing the design of sensor networks distributed along the structure [16-18].

The interpretation of the ultrasonic signals is complex when the propagation occurs in mechanical structures that behave as waveguides, as the case of a simple metallic plate due to the appearance of various guided waves modes [19]. Depending on the wavelength to plate thickness ratio, these waves are called Rayleigh or Lamb waves [20]. Moreover, Lamb waves may be dispersive, hindering the use of conventional signal processing techniques.

When a body is subject to stress, the velocity of ultrasonic waves propagating inside of it is altered. This is the acoustoelastic effect [21]. The most immediate manifestation of this phenomenon is time-of-flight change. The acoustoelastic effect can be used in order to determine the initial stress in a body by time-of-flight measuring [22,23] or using more sophisticated principles such as its applications to guided waves [10,24], and the correlation of the speckle images [25]. This principle can be very useful when concerns monitoring both strain and some kind of damage, as cracks [26]

The acoustoelastic effect can be addressed in the deformed or undeformed frames of reference. Which are commonly named initial and natural frames, respectively [27]. In the natural frame, the geometric variation due to the initial strain is already included in the velocity, whereas in the initial frame the velocity is related to the deformed geometry. The choice relies on the chosen problem. Addressing a problem in natural frame is more convenient when one has access to its natural configuration, as when the ultrasonic actuators are bonded on the body that goes through subsequent deformation. The initial 
frame is convenient when the deformed body is accessed as for analyzing residual stresses or when non-contact transducer are used.

The use of numerical approaches as finite element method (FEM) is a useful tool for ultrasonic waves propagation analysis [28]. Commercial FEM programs cannot straightly simulate the acoustoelastic effect. An alternative is to use an equivalent anisotropic medium by means of effective elastic constants (EEC) [29]. Addressing the acoustoelastic effect by means of EEC allows assembling an equivalent anisotropic stiffness tensor, which can be the input in commercial FEM simulation software. This approximation however introduces an inherent imprecision. There are few works simulating the acoustoelastic effect [30], specially on Lamb waves. [31-33].

The acoustoelastic theory applied to guided waves is a very new branch of study. Measurement of load by means of time-of-flight shift of Lamb modes is possible since Gandhi developed the theory of acoustoelasticity for Lamb waves in 2012 [34] and enabled the theoretical calculation of velocity variation as a function of stress [24]. In order to analytically obtain the velocity dependence on stress, the whole dispersion relationship has to be calculated for each mode. The velocity variation is complex, depends on the frequency and mode. The use of this theory in order to absolutely measure the initial stress requires using correct materials constants in order to precisely obtain the velocity change. Not only the elastic constants, as Young modulus and Poisson coefficient, or equivalently the Lamé constants, need to be known, but the third order elastic constants, as the Murnaghan constants [35] are also need to be known. There is however great divergence on the literature regarding the values of this constants for the same material [36-42].

If the exactly constants are not available the alternative is to use relative methods. For instance, time-of-flight shifts relative to a reference stress state. In this approach the use of cross-correlation for determining the time-of-flight shift is convenient and disseminated [43-45]. The time-reversal technique is also feasible for this usage $[46,47]$.

The time reversal process is a well-known method for obtaining focused acoustic waves in both time and space [48]. In the case of Lamb waves, the use of time reversed signals compensates the dispersion of each propagation mode, recompressing the signal and producing a focused signal at the reception point $[49,50]$. The time-reversal technique can be viewed as a physical crosscorrelation and introduces the advantage of high gain.

If acoustoelasticity of Lamb modes is used for stress measuring it is natural to look for exciting and sensing a single Lamb mode $[10,24,34]$. The use of many modes, that can be excited by a broadband pulse, can render the time- 
shift measurement quite a puzzling task to analyze due to mode overlapping and dispersion of different kinds for each mode. If one uses the time-reversal approach this is not necessarily true, as the technique recompress the signal regardless the number of modes, their dispersion characteristics, and even possible reflections [51]. Moreover, if many modes propagates with different velocity sensitivity to stress, then the focus amplitude decrease may occur in addition to time shift.

In an early work these effects on the time-reversal signal were verified [52, 53]. In that work the phenomenon was evaluated using a pair of conventional piezoelectric transducers, showing low gain and low sensitivity in the amplitude changes, and no theoretical approach by the acoustoelastic theory was adopted. Such a preliminary result encouraged the author to continue the research on this topic.

The main limitation in the preliminary implementation was the quasimonochromatic transfer function introduced by using conventional transducers. Thus, it has been initially investigated the use of wider band transducers. For this purpose 2-2 piezocomposite transducers were experimented, these transducers present wider frequency band than single ceramic transducers. It has been observed that wider band setups presented a higher amplitude sensitivity, showing correlation with the focusing energy efficiency of the time-reversal process, as presented in chapter 9. These results were published in $[54-56]$.

Then it has been investigated the application of signal processing algorithms in order to synthesize a more sensitive reference signal to perform the time-reversal process. This allows to obtain higher sensitivity in the timereversal signal for strain variations. Preliminary result on this research were published in [57].

The sequel of the research focused however on better understanding the acoutoelastic phenomenon in guided waves specially by addressing it in a formal mathematical background. This path has been chosen due to its nontrivial and motivating theory, presented in chapters 3 to 5, and also aiming to build up the necessary framework to simulate the phenomenon in commercial finite element software, assessed in chapter 6 . This is a contribution by itself and allow to enhance and evaluate the applicability of the time-reversal method through computational designing, as analyzed in chapter 8.

This thesis has then two main objectives. First, to investigate the acoustoelastic theory applied to Lamb waves, and then to understand its effect on the time reversal process, specially related to the system complexity (spectral content and amount of modes). Finally, to analyze the feasibility and 
limitations of using the time reversal focus characteristics in order to measure the strain.

In order to achieve them, the acoustoelasticity has been studied. The new branch of acoustoelasticity for Lamb waves has been thoroughly analyzed and its state of the art reviewed. The possibility of using effective elastic constants for determination of the velocity dependence on stress and the errors in this approximation has been investigated in both natural and initial frames of reference. The use of FEM in Ansys ${ }^{\circledR}$ has been validated for single mode under uniaxial tensile stress. Then the simulations of wideband multimode acoustoelastic Lamb waves was carried out in Ansys ${ }^{\circledR}$, the time reversal focusing ability has been verified; the effect of uniaxial load on the focuses has been investigated in some different scenarios. Finally, the numerical results were compared to the experiments performed in an aluminum plate where three pair of 2-2 piezocomposite transducers are bonded at its extremities.

This thesis is organized as follows:

- Chapter 1 introduces the problem and objectives.

- Chapter 2 briefly reviews the most significant aspects of Lamb Waves and present the signal processing techniques useful for dispersion analysis, as dispersion compensation and extraction, and for Lamb waves, as mode separation.

- Chapter 3 reviews the theory of acoustoelasticity and derive the wave equation in both the natural and initial frames of reference.

- Chapter 4 reviews the theory of acoustoelasticity applied to Lamb waves and the theoretical procedure for obtaining the velocity dispersion curves in a loaded medium by means of the partial wave technique. The simplification for axial loading is developed as well as the approximation for effective anisotropic medium by means of the effective elastic constants.

- Chapter 5 calculates the velocity dispersion curves for longitudinal loaded medium based on the theory of the preceding chapter. The calculations are performed for both natural and initial frame of reference. The validity of the EEC approximation is investigated.

- Chapter 6 simulates the fundamental Lamb wave modes in a loaded plate and compares the extracted dispersion curves in order to validate the simulation of acoustoelasticity by means of EEC.

- Chapter 7 reviews the Time Reversal theory and develops the theoretical effect of strain on the time reversal signal. 
- Chapter 8 simulates the time reversal of wideband multi-mode Lamb wave in a longitudinally loaded body. The effect of the initial stress on the time reversal signal is investigated in several scenarios.

- Chapter 9 presents the experiments performed with the piezocomposite transducers in an aluminum plate longitudinally loaded.

- Chapter 10 concludes the work.

- Appendix A demonstrates the symmetries on the second and third order stiffness tensors and on accoustoelastic tensors.

- Appendix B lists the published papers related to the thesis.

The main contributions of this thesis are (i) to clearly determine the validity of the EEC approximation for Lamb waves; (ii) to demonstrate the feasibility of using commercial FEM programs in order to simulate the load effect on Lamb wave propagation and point out its precision limits; (iii) to investigate and understand the effect of initial stress on the time reversal signal by means of simulation and experiments; and (iv) to analyze the feasibility of using this signal in order to monitor the stress state.

This work also points out an imprecision, and suggest the correction, on the literature reference for Lamb waves acoustoelasticity that introduces errors in the calculation of Lamb wave velocity at different conditions, for instance in other frame of reference. 


\section{2}

\section{Guided waves in plates}

Bulk waves propagate in a material volume where dimensions are big enough to ensure that surface reflections do not occur or can be neglected. In an uniform plate a wave front that propagates inside of it can hit the surfaces and then be reflected. The superposition of the many wave fronts, due to the many reflections, assembles a wave guide packet, called guided wave mode.

From a mathematical point of view, guided waves are ruled by the same differential equations as bulk waves. The difference lies on the boundary conditions. Guided waves must satisfy the boundary conditions at the surface, while bulk waves are free to propagate in an unbounded medium.

There are several kinds of guided waves. Between them, the Lamb waves are those that propagate in a plate, that when immersed in vacuum must obey null stress boundary conditions at the surfaces. The Lamb wave modes are composed by longitudinal and transverse shear waves that inter-convert among themselves. There are a countably infinite number of Lamb waves modes along the frequency spectrum. A very strong characteristic of this guided waves is to be dispersive. Dispersive waves are those such that the velocity changes as a function of frequency. Thus a pulse shape (that contains a non-unitary frequency component) changes its shape as it propagates.

In this section the most important equations and developments for Lamb wave and for the work sequel are exposed. The complete development can be found in well-known textbooks as Rose [14,58] and Achenbach [59]. At the end of the chapter some useful signal processing methods for guided waves analysis are presented.

\section{1}

\section{Wave equation for elastic media}

This section derives the equation of motion for elastic media, this is done under the assumption of infinitesimal strain. This development is based on three equations, the Newton's second law, the strain definition and the constitutive equation. 
Newton's second law is expressed in index notation by

$$
\frac{\partial \sigma_{i j}}{\partial x_{j}}+\rho f_{i}=\rho \frac{\partial^{2} u_{i}}{\partial t^{2}}
$$

Where $\boldsymbol{\sigma}$ represents the stress tensor, $\rho$ represents the material density, $\boldsymbol{u}$ is the displacement, $\boldsymbol{x}$ is the position vector, and $\boldsymbol{f}$ is the body force per mass unity applied to the material.

The second equation relates strain with displacement. The strain tensor, $\varepsilon$, is a second order tensor defined by the symmetrical part of the displacement gradient, as shown in $(2.2)$

$$
\varepsilon_{i j}=\frac{1}{2}\left(\frac{\partial u_{i}}{\partial x_{j}}+\frac{\partial u_{j}}{\partial x_{i}}\right) \quad .
$$

The third expression is the generalized Hooke's law that relates stress to strain through the stiffness tensor $\boldsymbol{C}$. This is expressed by

$$
\sigma_{i j}=C_{i j k l} \varepsilon_{k l}
$$

As fourth order tensor, the elastic tensor may have, a priori, $3^{4}=81$ independent components. However considering that the stress and strain tensors are symmetric, it can be concluded that the elastic tensor has a maximum of 21 independent components. Thus presenting the following symmetries.

$$
\begin{aligned}
C_{i j k l} & =C_{j i k l}, \\
C_{i j k l} & =C_{i j l k}, \\
C_{i j k l} & =C_{k l i j}
\end{aligned}
$$

More on the symmetries of the stiffness tensors is found in Appendix A.

For an isotropic medium the stiffness tensors are represented by [14]

$$
C_{i j k l}=\lambda \delta_{i j} \delta_{k l}+2 \mu I_{i j k l}
$$

where $\lambda$ and $\mu$ are, respectively, the first and second Lamé constants, $\delta$ is the Kroenecker delta function given by

$$
\delta_{i j}= \begin{cases}1 & , i=j \\ 0 & , i \neq j\end{cases}
$$

and the tensor I defined by

$$
I_{i j k l}=\frac{\delta_{i k} \delta_{j l}+\delta_{i l} \delta_{j k}}{2} .
$$

Applying (2.3) in (2.2) and then in (2.1) leads to 


$$
\begin{aligned}
\frac{\partial C_{i j k l} \frac{1}{2}\left(\frac{\partial u_{k}}{\partial x_{l}}+\frac{\partial u_{l}}{\partial x_{k}}\right)}{\partial x_{j}} & =\rho \frac{\partial^{2} u_{i}}{\partial t^{2}} \Rightarrow \\
\frac{1}{2}\left(C_{i j k l} \frac{\partial^{2} u_{k}}{\partial x_{l} \partial x_{j}}+C_{i j k l} \frac{\partial^{2} u_{l}}{\partial x_{k} \partial x_{j}}\right) & =\rho \frac{\partial^{2} u_{i}}{\partial t^{2}},
\end{aligned}
$$

where the body forces are considered null. The symmetry (2.4b) allows permutation of indexes $k$ and $l$, thus

$$
C_{i j k l} \frac{\partial^{2} u_{k}}{\partial x_{l} \partial x_{j}}=\rho \frac{\partial^{2} u_{i}}{\partial t^{2}} .
$$

This is the wave equation that admits solution as

$$
u_{i}=U_{i} e^{i\left(\kappa_{m} x_{m}-\omega t\right)},
$$

where $\boldsymbol{\kappa}$ is the wavevector and $\omega$ the angular frequency, which is related to the ordinary frequency by

$$
\omega=2 \pi f
$$

Considering the direction of propagation as $x_{1}$, the wave in the component $u_{1}$ is classified as a primary, or longitudinal, wave, because it is polarized in the direction of propagation, and called $\mathrm{P}$ wave. A wave in $u_{2}$ is a secondary, or transverse, horizontal wave; called SH. Finally, a wave in which the displacement vector is $u_{3}$ is a secondary, or transverse, vertical wave; called SV.

For isotropic medium the wave velocity is independent on the propagation direction. There are however different velocities depending on the polarization. The velocity $c_{L}$ is the veloctiy of $\mathrm{P}$ waves and $c_{T}$ is the velocity of $\mathrm{SH}$ and $\mathrm{SV}$ waves. These are given by

$$
\begin{aligned}
c_{L} & =\sqrt{\frac{\lambda+2 \mu}{\rho}}, \\
c_{T} & =\sqrt{\frac{\mu}{\rho}} .
\end{aligned}
$$

\section{2}

\section{Lamb waves}

In this section it is studied the particular case of a parallel face plate arranged as shown in Fig. 2.1.a. The plate thickness is $d=2 h$, the surfaces of the plate lie in the planes $x_{3}=h$ and $x_{3}=-h$. Lamb waves are guided by multiple reflection on the top and bottom surface of the plate, propagating longitudinally, i.e. in $x_{1}$ direction, as illustrated in Fig. 2.1.b.

This kind of wave respect the boundary condition of free stress on the 


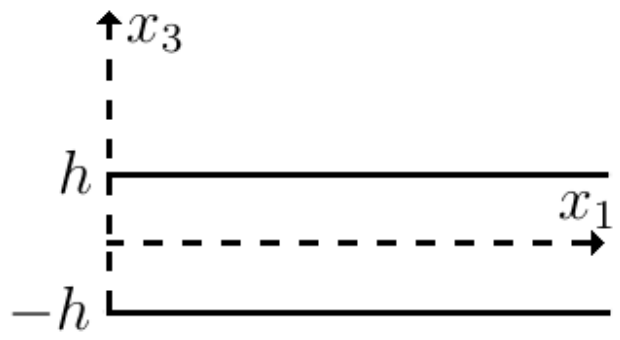

(a)

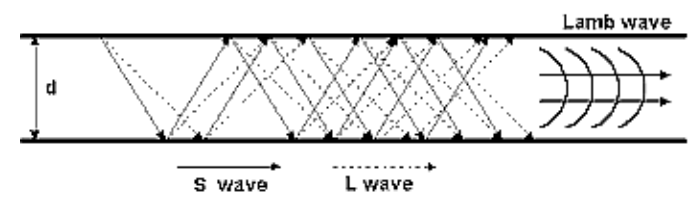

(b)

Figure 2.1: Plate geometry (a), adapted from [14], and Lamb waves generation (b), adapted from [60].

plate surfaces, i.e.,

$$
\left.\sigma_{i 3}\right|_{x_{3}= \pm h}=0
$$

There are some methods for solving this problem. For an isotropic medium the most common is the method of displacement potentials [59]. For anisotropic medium the partial wave technique or the propagation matrix are more adequate. The partial wave technique is further explained in chapter 4; a detailed explanation on the propagation matrix method can be found in Braga and Hermann [61].

For isotropic medium the SH waves decouple from $\mathrm{P}$ and $\mathrm{SV}$ waves. It is then possible to occur the mode conversion between $\mathrm{P}$ and $\mathrm{SV}$, which does not occur between $\mathrm{P}$ and $\mathrm{SH}$ or $\mathrm{SV}$ and $\mathrm{SH}$. The Lamb waves are commonly restricted to P-SV waves guided inside the plate.

There are two kinds of Lamb waves modes, symmetric and antisymmetric. This classification stands for $u_{1}$ displacement related to the plane $x_{3}=0$. Fig. 2.2.a shows the modes shapes.

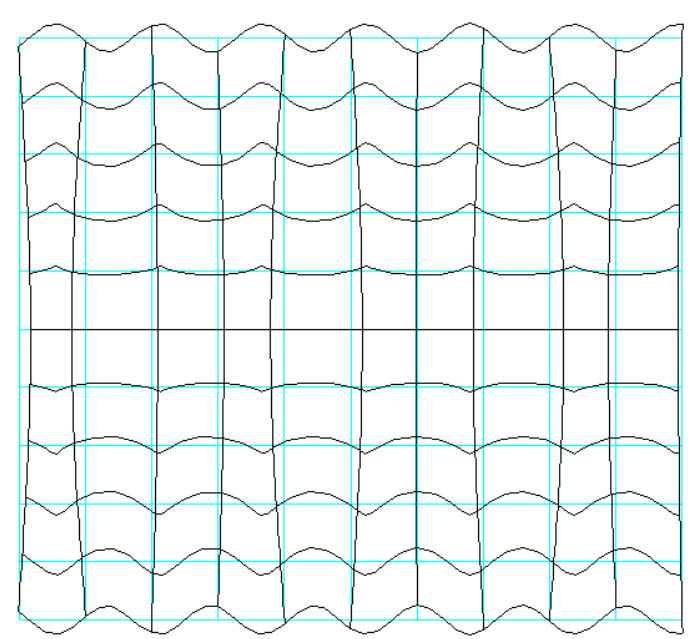

(a)

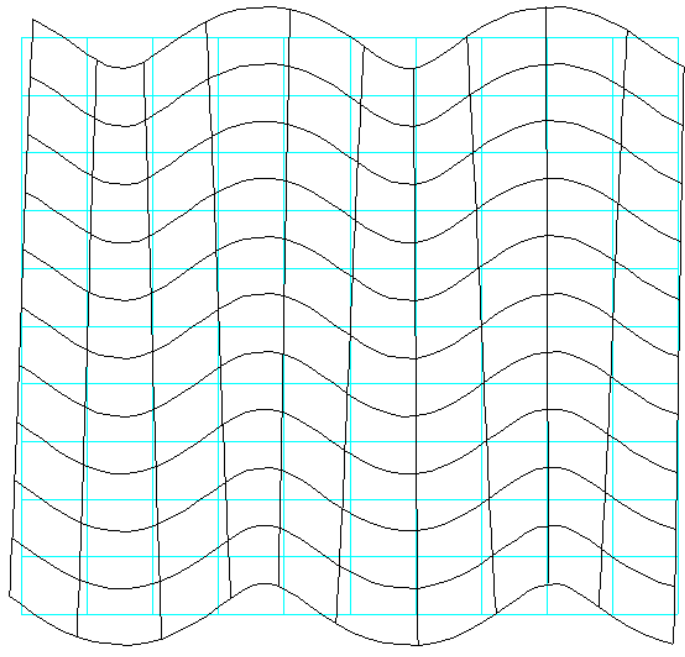

(b)

Figure 2.2: First order modes shape. (a) symmetric (b) antisymmetric. Adapted from Disperse software [62] 
For isotropic medium the solutions are given by the well-known RayleighLamb equations, (2.14) for symmetric modes, and (2.15) for antisymmetric modes. Each solution consists of a pair of wave number $(\kappa)$ and angular frequency $(\omega)$ that represents a possible propagation condition $[19,58]$. The equations assume the following expression

$$
\begin{aligned}
\frac{\tan (q h)}{\tan (p h)} & =-\frac{4 \kappa^{2} p q}{\left(q^{2}-\kappa^{2}\right)^{2}} \quad \text { and } \\
\frac{\tan (q h)}{\tan (p h)} & =-\frac{\left(q^{2}-\kappa^{2}\right)^{2}}{4 \kappa^{2} p q}
\end{aligned}
$$

where $p^{2}=\omega^{2} / c_{L}^{2}-\kappa^{2}, q^{2}=\omega^{2} / c_{T}^{2}-\kappa^{2}$. There is propagation only for the pairs, $\omega$ and $\kappa$, that solves the equations above.

At a given angular frequency $\omega$ (or frequency, $f$ ), there can be several solutions for the wave number $\kappa$. Related to the different modes. There is no analytical solution for (2.14) and (2.15). Solving them is only possible numerically.

The wave is said to be dispersive if either the group or phase velocity depend on frequency. This happens for Lamb waves. The phase velocity represents the velocity of each individual phase, whereas the group velocity is the velocity of the wave packet, it is also the velocity of energy transportation. Those velocities are given, respectively, by

$$
\begin{gathered}
c_{p h}(f)=\frac{\omega}{\kappa}=\frac{2 \pi f}{\kappa} \quad \text { and } \\
c_{g r}(f)=\frac{d \omega}{d \kappa}=2 \pi \frac{d f}{d \kappa} .
\end{gathered}
$$

The group velocity can be expressed in term of the phase velocity by

$$
c_{g r}=\frac{c_{p h}^{2}}{c_{p h}-\omega \frac{\partial c_{p h}}{\partial \omega}}=\frac{c_{p h}^{2}}{c_{p h}-f \frac{\partial c_{p h}}{\partial f}} .
$$

The modes distribution along the frequency range can be observed in the dispersion curves. These are commonly expressed as a function of the frequency times the plate thickness. Fig. 2.3 shows the dispersion curve for group and phase velocity for an aluminum plate. Only the fundamental symmetrical and antisymmetrical modes, S0 and A0, respectively, are present for all frequencies. Higher order modes are not present for low frequencies, they can only propagate from their respective initial cut-off frequencies. As the frequency increases, more modes arise, and there are a countably infinite number of them.

The velocity behavior for each mode can vary widely with the frequency. Regions where the velocity is very dependent on the frequency are more dispersive, as for example from 0 to $0.6 \mathrm{MHz} \times \mathrm{mm}$ for the A0 mode, from 1.5 to $3 \mathrm{MHz} \times \mathrm{mm}$ for the S0 mode, and for higher order modes around their 


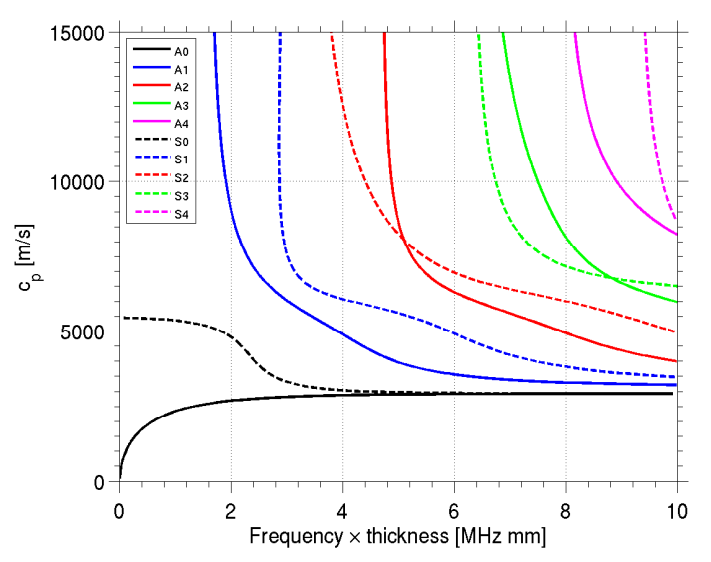

(a)

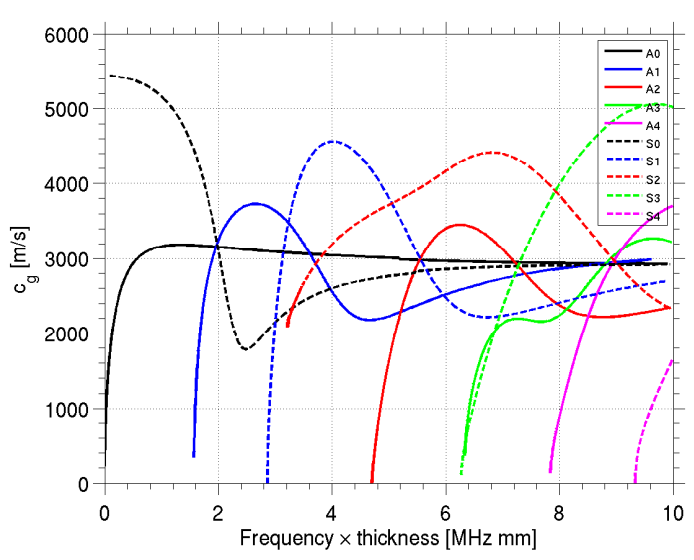

(b)

Figure 2.3: Phase (a) and group (b) velocity dispersion curves for an aluminum plate. Solid lines stands for antissymetrical modes and dashed lines for symmetrical. Black lines are fundamental modes, blue first order modes, red second order, green third order and pink forth order modes.

cut-off frequencies, among other regions observable in Fig. 2.3. For these, a nonmonochromatic traveling pulse has its shape distorted during the propagation. Due to a broadband excitation, many modes can propagate in the plate, each of them arriving at the measuring position at different times, with a different relative phase; their pulses may overlap in time domain, depending on the receiver position, rendering signal interpretation complicated.

\section{3}

\section{Signal processing for guided waves analysis}

In this section useful signal processing methods for guided waves analysis are presented, such as dispersion characterization and compensation as well as mode identification and separation. Only those methods which are used in this work are explained.

\subsection{1}

\section{Extracting dispersion from sensed signal}

The are several techniques dealing with extracting dispersion parameters, such as phase and group velocities or wavenumber, from sensed wave signals. These can be based on two-dimension Fourier transform [63-65], phase spectral analysis [66], time-frequency decompositions such as short-time Fourier transform [67], wavelet transform [67-72], S transform [73,74] and pseudo WignerVille distribution $[67,75]$. The first two, i.e. two-dimension Fourier transform and phase spectral analysis are explained below. 


\subsubsection{1}

\section{Two-dimension Fourier transform}

The most straight technique is basically to perform a two-dimension Fourier transform (2DFT) of a a time-space dependent signal, say $s(x, t)$, where $t$ represents the time variable and $x$ the space one. This signal $s(x, t)$ may represent any possible dynamical response (e.g., strain, stress or displacement) which are easy obtained in simulation, or, in real cases, electric signals sensed by transducers. In the latter case many sensor should be distributed on the specimen in order to obtain an adequate spatial sampling (respecting Nyquist criterion [76]).

The one-dimension Fourier Transform (FT) of a time dependent signal, $s(t)$, is defined as [76]

$$
\hat{s}(\omega)=\int_{-\infty}^{\infty} s(t) e^{-i \omega t} d t
$$

The transform of $s(t)$, i.e $\hat{s}(\omega)$ or $\hat{s}(f)$, represents the signal in the frequency domain, and roughly speaking, shows the contribution of each exponential oscillation $\left(e^{i \omega t}\right)$ in the signal.

The 2DFT of $s(x, t)$ is the extension for FT to the $\mathbb{R}^{2}$, defined as [77]

$$
\hat{s}(\omega, k)=\int_{-\infty}^{\infty} \int_{-\infty}^{\infty} s(t) e^{-i(\omega t+k x)} d t d x
$$

where $k$ is the angular spatial frequency. The 2DFT of a signal represent it in the $\omega \times k$ plane, and thus shows the contribution of each double exponential in the signal. If one sets $\kappa=-k$ then the double exponential becomes the wave function, $e^{i(\kappa x-\omega t)}$, where $\kappa$ is the wavenumber. Ideally $\hat{s}(\omega, \kappa)$ of a wave signal is non-zero only for those $(\omega, \kappa)$ pairs that correspond to a wave solution contained in the signal. Thus, if a signal arising from wave propagation is available as a function of both, time and space, one can obtain its wavenumber $\times$ frequency dispersion characteristics by performing the 2DFT and simply flipping the spatial frequency variable then, i.e by setting $\kappa=-k$.

The dispersion curve can be associated with the maxima on amplitude spectra as done in $[63,64]$. The major disadvantage of this technique is the necessity of capturing the signal as a function of position, in the adequate sampling rate. 


\subsubsection{2}

\section{Phase spectral analysis}

This technique was proposed by Sachse and Pao [66]. It operates with sensed signals from two different position along the propagation path, say $s_{1}(t)$ and $s_{2}(t)$, sensed at $x_{1}$ and $x_{2}$ respectively, where $x_{1}<x_{2}$; let $\ell=x_{2}-x_{1}$ be the distance between position 1 and 2 . It basically consists of assuming that the Fourier spectrum of the signal at $x_{2}$ is a phase shifted and attenuated version of the one in $x_{1}$, thus

$$
\hat{s}_{2}(\omega)=\hat{s}_{1}(\omega) e^{-i \kappa(\omega) \ell} e^{-\alpha(\omega) \ell},
$$

where $\kappa(\omega)$ is the frequency dependent wavenumber and $\alpha(\omega)$ the frequency dependent attenuation.

Observe that, if neither $\kappa$ nor $\alpha$ are frequency dependent in (2.21), then this is equivalent, in the time domain, to a simple time shift, i.e.,

$$
s_{2}(t)=s_{1}(t-\ell / c) e^{-\alpha \ell},
$$

where $c$ is the phase velocity defined in (2.16). This a direct consequence of the time-shift property of the Fourier transform [76].

Writing the spectra as the product of the amplitude and phase, (2.21) becomes

$$
\left|\hat{s}_{2}(\omega)\right| e^{-i \phi_{2}(\omega)}=\left|\hat{s}_{1}(\omega)\right| e^{-i \phi_{1}(\omega)} e^{-i \kappa(\omega) \ell} e^{-\alpha(\omega) \ell},
$$

where $\phi_{1}(\omega)$ and $\phi_{2}(\omega)$ are the phases of $\hat{s}_{1}(\omega)$ and $\hat{s}_{2}(\omega)$, respectively. As both the amplitude and phase equalities must hold in (2.23), then this can be split

$$
\begin{aligned}
\left|\hat{s}_{2}(\omega)\right| & =\left|\hat{s}_{1}(\omega)\right| e^{-\alpha(\omega) \ell}, \\
\phi_{2}(\omega) & =\phi_{1}(\omega)+\kappa(\omega) \ell
\end{aligned}
$$

The wavenumber can be found by rearranging (2.24b) as

$$
\kappa(\omega)=\frac{\phi_{2}(\omega)-\phi_{1}(\omega)}{\ell},
$$

or, equivalent, by using (2.16) one can calculate the phase velocity as

$$
c_{p h}(\omega)=\frac{\omega \ell}{\phi_{2}(\omega)-\phi_{1}(\omega)} .
$$

This last equation states that by knowing the Fourier transform of two sensed signals and the distance between them, the dispersion relationship can be obtained by the phase different of their Fourier transform.

One important remark concerning this technique is that it is supposed that the signals are composed of just a single mode with no reflection. If the signal time-trace present more than one mode or any reflection one can use 
this only if they are resolved in time, by setting a time window that keeps only the echo of interest. Otherwise, if echoes overlap in time, one should not use this, because (2.21) do not hold any more.

\subsection{2}

\section{Dispersion compensation and mode separation}

Compensating the dispersion effect is possible if one knows the theoretical dispersion curve. It basically consists of multiplying the Fourier transform of a signal received at a certain distance $\left(\hat{s}_{1}(\omega)\right)$ from the source by a phase factor that correspond to the back propagation operator, i.e.,

$$
\hat{s}(x, \omega)=\hat{s}_{1}(\omega) e^{+i \kappa(\omega) x},
$$

where $\hat{s}_{1}(\omega)$ is the Fourier transform of the signal sensed, and $\hat{s}(x, \omega)$ is the artificially back propagated spectrum of the signal for a distance $x$. Computing the inverse Fourier transform of (2.27) yields to

$$
s(x, t)=\frac{1}{2 \pi} \int_{-\infty}^{\infty} \hat{s}_{1}(\omega) e^{i \kappa(\omega) x} e^{i \omega t} d \omega .
$$

If the distance $x$ is set as a variable then one has a function of both time and space; evaluating it at the instant $t=0$ the resulting space dependent function is

$$
s(x)=\frac{1}{2 \pi} \int_{-\infty}^{\infty} \hat{s}_{1}(\omega) e^{i \kappa(\omega) x} d \omega .
$$

This last space dependent signal is a compressed pulse in the position corresponding to the source, as the original signal was applied in that position. By this process one transforms a time dependent disperse signal in a space dependent signal in which dispersion is compensated.

This technique was proposed by Sicard et al. [78] and complemented by Wilcox [79] who minutely explained the numerical implementation. Imprecision in the theoretical dispersion curves leads to failure in the compensation. Wilcox [79] showed that error in the phase velocity, as what happens if incorrect material properties are used, implies predominantly in spatial shift; whereas error in the frequency $\times$ thickness axis, due to adopting incorrect thickness for instance, can imply in no compensation at all.

The technique exposed above consider that the signal is composed by a single mode. Xu et al. [80] proposed a similar technique for multimodal signals in order to identify and separate them.

If (2.28) is evaluated at the origin position, say $\ell$, then a time dependent signal is kept, as 


$$
s(t)=\frac{1}{2 \pi} \int_{-\infty}^{\infty} \hat{s}_{1}(\omega) e^{i \kappa(\omega) \ell} e^{i \omega t} d \omega .
$$

In the case where the original excitation was sharp in time, as a spike, this compensation, based in time dependent signal, recompress the signal into a sharp one.

Xu's idea is to apply (2.30) to a signal that can contain several modes, but using the wavenumber function, $\kappa(\omega)$, of just a single mode of interest. If this mode is contained in the signal, then a sharp pulse appears at $t=0$. The remainder of the signal, composed by the other modes, when passes through (2.30) results in low amplitude and long duration signal around the sharp pulse, because the compensation simply does not match to those other modes.

Identification can be done by processing (2.30) to all modes that can possibly compose the signal; acute peak at the origin occurs only for the modes that are contained in the signal. This technique relies on knowing the exact material properties (correct dispersion curve), propagated length and plate thickness.

Mode separation is also feasible. When compression is complete, a time window around the origin (with time length of about the original pulse) is used to nullify the content outside of it. Forward propagation is then done with the windowed signal, resulting in a signal composed of this specific mode only.

A variation of this technique is to artificially calculate a signal that is the result of spike that is back propagated for a certain distance, i.e., a spike with reversal dispersion. This signal is used to excite the actuator. The signal will naturally propagate and disperse the exactly amount in order to recover the original spike. This idea was first used by Yamasaki [81] in steel wire and formally proposed in wideband formulation for plates by $\mathrm{Xu}$ et al. [82]. This is similar to the time-reversal principle, however the signal used for excitation is calculated by means of a known dispersion curve, instead of measured. 


\section{3}

\section{Acoustoelastic equations of motion}

The acoustoelasticity studies the variation of the elastic waves velocity in bodies subject to an initial stress state [83]. The acoustoelastic theory consists of non-linear relationship between stress and strain that rules the dynamic response superimposed to the initial pre-deformation [84].

The modern theory of acoustoelasticity was completed by the general theory of finite deformation presented by Murnaghan [35], in which the third order elastic constant was introduced. One of the first works to calculate the velocity of ultrasonic bulk waves as a function of the stress was done by Hughes and Kelly [21].

The effect of pre-strees on the propagation of acoustic waves can be treated by the theory of exact non-linear elasticity, which can be found in well-known literature [84,85]. It relies on the proper strain energy density function that describes the constitutive relationship of a given material [86]. A comprehensive list of the most relevant strain energy functions is available in chapter two of De Pascalis thesis [87]. This approach is general and allows one to consider large deformation [88] and is often used to describe soft solids [89].

On the other hand, the theory of weakly non-linear elasticity considers the strain energy function expanded, typically, up to third order terms. This approach is commonly treated in a more straightforward, but less general, development which is as well consolidated [83,90-92]. It is employed to describe the acoustoelastic effect in ordinary stiff solids, as metals, with small pre-stress, and experimentally used to measure applied and residual stresses through ultrasonic techniques [92]. There are also some other variations, as for instance, the development of the acoustoelasticity in non-homogenous media [93, 94].

The approach adopted here is the one develop by Pao and Gamer [91], within the eakly non-linear elasticity, whose work derives the equations of motion and, by some approximations, linearizes them for the initial stress state. In other words, an equivalent stiffness and constitutive tensor arises as a function of the initial stress, which act as a stiffener for a new equivalent medium. The main points are reviewed; the most important derivations are analyzed in detail here because in that work some important equations are not thoroughly developed. 
The main objective of this chapter is to derive the acoustoelastic tensors used in the wave equation and in the incremental constitutive equation; these are necessary to obtain the dispersion curves for Lamb wave propagating in a pre-stressed plate, presented in the next chapter. The derivations are done in both the natural and initial frame of reference in order to consolidate the theoretic framework of elastic wave under initial stress.

\section{1}

\section{Finite deformation elasticity}

Three states of reference are required in order to develop the acoustoelastic theory of motion. A body is deformed from its natural (undeformed or unstressed) state to the statically deformed state, called the initial state. Wave motion is superimposed to the initial state leading to the final state. The following notation is used for representing the coordinates of a material point at each of the states. Greek letters refer to the natural state $\left(\xi_{\alpha}, \alpha=1,2,3\right)$; Capital roman letters to the initial state $\left(X_{I}, I=1,2,3\right)$; and primed lower case roman letters to the final state $\left(x_{i}^{\prime}, i=1,2,3\right)$. Deformations between the states are classified as the initial deformation from natural to initial states, final deformation from natural to final state, and the incremental deformation from initial to final. The corresponding displacements are given respectively by

$$
\begin{aligned}
\mathbf{u}^{i} & =\mathbf{X}-\xi, \\
\mathbf{u}^{f} & =\mathbf{x}^{\prime}-\xi, \\
\mathbf{u} & =\mathbf{x}^{\prime}-\mathbf{X}=\mathbf{u}^{f}-\mathbf{u}^{i},
\end{aligned}
$$

the initial deformation is the only static deformation, both final and incremental are dynamic as the final state is time varying. Fig. 3.1 illustrates the states and deformations.

The equations of motion can be derived in both natural or initial frames of reference. The initial frame is the one where the body actually resides. That is, the velocity related to the initial frame takes into account the actual distance per propagated time. On the other hand, the problem treated in the natural frame considers the natural length per propagated time, and thus the calculated velocity is not the physical wave velocity. One may see the latter as an artificial velocity. Nevertheless, this can be very handy depending on problem. The propagation phenomenon is separated from the statical deformation (or stress) when addressed at the initial frame, whereas when addressed at the natural frame the static deformation is already embedded in 


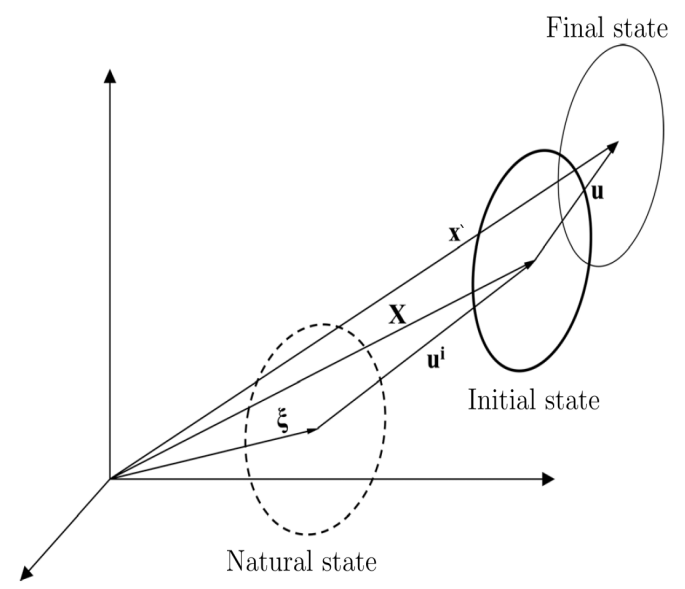

Figure 3.1: Three states configuration. Natural $\xi$, initial $\mathbf{X}$, and final states $\mathbf{x}^{\prime}$. Adapted from [34]

the formulation. The choice of which frame of reference is more convenient depends on the problem. The natural frame is more convenient when one has access to its natural configuration, as in experiments where the wave actuators are fixed on the body that goes through successive deformation. The initial frame is better suited when it is not possible to access the natural state of a material, due to residual stresses, or when non-contact transducers are used with fixed distance between emitter and receiver, for instance.

The theory of acoustoelasticity assumes that the body is hyperelastic at all the three states. It also assumes that the strain energy function, $W$, is well approximated by two terms polynomial expansion in strain, as

$$
W=\frac{1}{2} C_{\alpha \beta \gamma \delta} E_{\alpha \beta} E_{\gamma \delta}+\frac{1}{6} C_{\alpha \beta \gamma \delta \zeta \eta} E_{\alpha \beta} E_{\gamma \delta} E_{\zeta \eta}
$$

where $E$ is the Lagrangian strain at initial $\left(E^{i}\right)$ or final $\left(E^{f}\right)$ states, $C_{i j k l}$ and $C_{i j k l m n}$ are the forth and sixth order stiffness tensor, respectively.

For an isotropic medium the forth order stiffness tensors is given by (2.5), and the sixth order is [35]

$$
\begin{aligned}
C_{i j k l m n} & =(2 l-2 m+n) \delta_{i j} \delta_{k l} \delta_{m n} \\
& +(2 m-n)\left(\delta_{i j} I_{k l m n}+\delta_{k l} I_{m n i j}+\delta_{m n} I_{i j k l}\right) \\
& +\frac{n}{2}\left(\delta_{i k} I_{j l m n}+\delta_{i l} I_{j k m n}+\delta_{j k} I_{i l m n}+\delta_{j l} I_{i k m n}\right)
\end{aligned}
$$

where $l, m$ and $n$ are the Murnaghan constants, $\delta$ is defined in (2.6) and $\mathbf{I}$ in (2.7).

The second Piola-Kirchhoff stress is given by the energy derivative,

$$
s_{\alpha \beta}^{i \text { or } f}=\frac{\partial W}{\partial E_{\alpha \beta}^{i o r f}},
$$


and thus the stress-strain relation in an hyperelastic medium is given by

$$
s_{\alpha \beta}^{i \text { orf }}=C_{\alpha \beta \gamma \delta} E_{\gamma \delta}^{i \text { or } f}+\frac{1}{2} C_{\alpha \beta \gamma \delta \zeta \eta} E_{\gamma \delta}^{i \text { orf } f} E_{\zeta \eta}^{i \text { or } f} .
$$

The second Piola-Kirchhoff stress tensor describes the stresses relative to a original reference; in this notation, the natural. Roughly speaking, the implicit area is taken from the natural coordinates. These tensors can be at the initial $\left(s_{\alpha \beta}^{i}\right)$ or final states $\left(s_{\alpha \beta}^{f}\right)$. The initial one relates the stresses involved in the initial deformation; the one necessary from taking the body from the natural to the initial state. The final one relates the stresses involved in bringing the body from natural to final state. It is also possible to define the second Piola-Kirchhoff stress related to the initial state. Thus $s_{I J}^{f}$ is the stress used to take the body from the natural to the final state, per initial area.

The Cauchy stress tensor is also useful, here symbolized by $\mathbf{t}$. It describes the stresses relative to the actual reference, either initial or final here. Hence, $t_{I J}^{i}$ relates the stresses involved to take the body from the natural to the initial state, related (per area) to the initial states, whereas $t_{i j}^{f}$ relates the stresses involved to take the body from the natural to the final state, related to the final state.

These tensors are related through a coordinate transformation by

$$
t_{i j}=\frac{1}{\operatorname{det}(\mathbf{F})} F_{i l} F_{j k} s_{l k}
$$

where $\mathbf{F}$ is the Gradient of Deformation that is defined as the derivative of the actual coordinate by the original. Using the notation adopted here it is

$$
F_{I \alpha}=\frac{\partial X_{I}}{\partial \xi_{\alpha}}
$$

Its determinant can be showed to be the ratio of the volume in the actual to the original states [95], or if the mass is constant, the ratio of the density in the original to the actual states. Thus the following relationship can be stated for the configuration of interest here:

$$
\begin{aligned}
t_{I J}^{i} & =\frac{\rho^{i}}{\rho^{0}} \frac{\partial X_{I}}{\partial \xi_{\alpha}} \frac{\partial X_{J}}{\partial \xi_{\beta}} s_{\alpha \beta}^{i}, \\
t_{i j}^{f} & =\frac{\rho^{f}}{\rho^{i}} \frac{\partial x_{i}^{\prime}}{\partial X_{K}} \frac{\partial x_{j}^{\prime}}{\partial X_{L}} s_{K L}^{f} \\
t_{i j}^{f} & =\frac{\rho^{f}}{\rho^{0}} \frac{\partial x_{i}^{\prime}}{\partial \xi_{\alpha}} \frac{\partial x_{j}^{\prime}}{\partial \xi_{\beta}} s_{\alpha \beta}^{f}, \\
s_{K L}^{f} & =\frac{\rho^{i}}{\rho^{0}} \frac{\partial X_{K}}{\partial \xi_{\alpha}} \frac{\partial X_{L}}{\partial \xi_{\beta}} s_{\alpha \beta}^{f},
\end{aligned}
$$

where $\rho^{0}, \rho^{i}, \rho^{f}$ are the density in the natural, initial and final frames, 
respectively. Equation (3.8d) is a consequence of (3.8b) and (3.8c). Note that none of the above stresses are incremental (take forces from initial to final). The incremental stresses are obtained by subtraction,

$$
T_{\alpha \beta}=s_{\alpha \beta}^{f}-s_{\alpha \beta}^{i}
$$

is the stress to take the body from initial to final states related to the natural state, whereas

$$
T_{I J}=s_{I J}^{f}-t_{I J}^{i}
$$

is the stress to take the body from initial to final states related to the initial state.

\subsection{1}

\section{Equilibrium condition and motion equation}

The equilibrium condition for the initial displacement in the natural and initial frame respectively are

$$
\begin{aligned}
\frac{\partial}{\partial \xi_{\beta}}\left(s_{\alpha \beta}^{i} \frac{\partial X_{\gamma}}{\partial \xi_{\alpha}}\right) & =0 \\
\frac{\partial t_{J L}^{i}}{\partial X_{L}} & =0
\end{aligned}
$$

The equation of motion for the final displacement in the natural and initial frame respectively are

$$
\begin{aligned}
\frac{\partial}{\partial \xi_{\beta}}\left(s_{\alpha \beta}^{f} \frac{\partial x_{\gamma}^{\prime}}{\partial \xi_{\alpha}}\right) & =\rho^{0} \frac{\partial^{2} x_{\gamma}^{\prime}}{\partial t^{2}}, \\
\frac{\partial}{\partial X_{L}}\left(s_{K L}^{f} \frac{\partial x_{I}^{\prime}}{\partial X_{K}}\right) & =\rho^{i} \frac{\partial^{2} x_{I}^{\prime}}{\partial t^{2}} .
\end{aligned}
$$

It is worth to highlight that the equations of motion must consider the final stress, and not the incremental one. Using just the incremental stress would mean neglecting the influence of the initial stress on the motion condition.

Applying the (3.1a) into (3.11a)

$$
\begin{aligned}
\frac{\partial}{\partial \xi_{\beta}}\left(s_{\alpha \beta}^{i} \frac{\partial\left(u_{\gamma}^{i}+\xi_{\gamma}\right)}{\partial \xi_{\alpha}}\right) & = \\
\frac{\partial}{\partial \xi_{\beta}}\left[s_{\alpha \beta}^{i}\left(\frac{\partial u_{\gamma}^{i}}{\partial \xi_{\alpha}}+\delta_{\gamma \alpha}\right)\right] & = \\
\frac{\partial}{\partial \xi_{\beta}}\left(s_{\alpha \beta}^{i} \frac{\partial u_{\gamma}^{i}}{\partial \xi_{\alpha}}+s_{\gamma \beta}^{i}\right) & =0 ;
\end{aligned}
$$

applying (3.1b), (3.1c) and (3.9) into (3.12a) 


$$
\begin{aligned}
\frac{\partial}{\partial \xi_{\beta}}\left(s_{\alpha \beta}^{f} \frac{\partial\left(u_{\gamma}^{f}+\xi_{\gamma}\right)}{\partial \xi_{\alpha}}\right) & =\rho^{0} \frac{\partial^{2} x_{\gamma}^{\prime}}{\partial t^{2}} \Rightarrow \\
\frac{\partial}{\partial \xi_{\beta}}\left[s_{\alpha \beta}^{f}\left(\frac{\partial u_{\gamma}^{f}}{\partial \xi_{\alpha}}+\delta_{\gamma \alpha}\right)\right] & =\rho^{0} \frac{\partial^{2} u_{\gamma}}{\partial t^{2}} \Rightarrow \\
\frac{\partial}{\partial \xi_{\beta}}\left(s_{\alpha \beta}^{f} \frac{\partial u_{\gamma}^{f}}{\partial \xi_{\alpha}}+s_{\gamma \alpha}^{f}\right) & =\rho^{0} \frac{\partial^{2} u_{\gamma}}{\partial t^{2}} \Rightarrow \\
\frac{\partial}{\partial \xi_{\beta}}\left(s_{\alpha \beta}^{f} \frac{\partial\left(u_{\gamma}^{i}+u_{\gamma}\right)}{\partial \xi_{\alpha}}+s_{\gamma \alpha}^{f}\right) & =\rho^{0} \frac{\partial^{2} u_{\gamma}}{\partial t^{2}} \Rightarrow \\
\frac{\partial}{\partial \xi_{\beta}}\left(s_{\alpha \beta}^{f} \frac{\partial u_{\gamma}^{i}}{\partial \xi_{\alpha}}+s_{\alpha \beta}^{f} \frac{\partial u_{\gamma}}{\partial \xi_{\alpha}}+s_{\gamma \alpha}^{f}\right) & =\rho^{0} \frac{\partial^{2} u_{\gamma}}{\partial t^{2}} \Rightarrow \\
\left.\frac{\partial}{\partial \xi_{\beta}}\left(T_{\alpha \beta}+s_{\alpha \beta}^{i}\right) \frac{\partial u_{\gamma}^{i}}{\partial \xi_{\alpha}}+\left(T_{\alpha \beta}+s_{\alpha \beta}^{i}\right) \frac{\partial u_{\gamma}}{\partial \xi_{\alpha}}+T_{\gamma \beta}+s_{\gamma \beta}^{i}\right) & =\rho^{0} \frac{\partial^{2} u_{\gamma}}{\partial t^{2}} \Rightarrow \\
\frac{\partial}{\partial \xi_{\alpha}}\left(s_{\alpha \beta}^{i} \frac{\partial u_{\gamma}^{i}}{\partial \xi_{\alpha}}+T_{\alpha \beta} \frac{\partial u_{\gamma}}{\partial \xi_{\alpha}}+s_{\alpha \beta}^{i} \frac{\partial u_{\gamma}}{\partial \xi_{\alpha}}+T_{\gamma \beta}+s_{\gamma \beta}^{i}\right) & \left.=\rho^{0} \frac{\partial^{2} u_{\gamma}}{\partial \xi_{\alpha}}+s_{\alpha \beta}^{i} \frac{\partial u_{\gamma}^{i}}{\partial \xi_{\alpha}}+s_{\alpha \beta}^{i} \frac{\partial u_{\gamma}}{\partial \xi_{\alpha}}+T_{\gamma \beta}+s_{\gamma \beta}^{i}\right)
\end{aligned}
$$

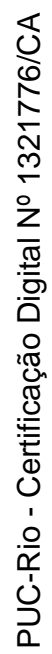

where the term containing the incremental stress times the incremental displacement is neglected because involves the product of two incremental entities and thus a small term of higher order. Subtracting (3.13) from (3.14) results in the equation of motion for the incremental displacement in natural frame of reference :

$$
\frac{\partial}{\partial \xi_{\beta}}\left(s_{\alpha \beta}^{i} \frac{\partial u_{\gamma}}{\partial \xi_{\alpha}}+T_{\alpha \beta} \frac{\partial u_{\gamma}^{i}}{\partial \xi_{\alpha}}+T_{\gamma \beta}\right)=\rho^{0} \frac{\partial^{2} u_{\gamma}}{\partial t^{2}} .
$$

The same is done for the initial frame. Applying (3.1c) and (3.10) into (3.12b) leads to

$$
\begin{aligned}
\frac{\partial}{\partial X_{L}}\left(s_{K L}^{f} \frac{\partial\left(u_{I}+X_{I}\right)}{\partial X_{K}}\right) & =\rho^{i} \frac{\partial^{2} x_{I}^{\prime}}{\partial t^{2}} \Rightarrow \\
\frac{\partial}{\partial X_{L}}\left[s_{K L}^{f}\left(\frac{\partial u_{I}}{\partial X_{K}}+\delta_{I K}\right)\right] & =\rho^{i} \frac{\partial^{2} u_{I}}{\partial t^{2}} \Rightarrow \\
\frac{\partial}{\partial X_{L}}\left(s_{K L}^{f} \frac{\partial u_{I}}{\partial X_{K}}+s_{I L}^{f}\right) & =\rho^{i} \frac{\partial^{2} u_{I}}{\partial t^{2}} \Rightarrow \\
\frac{\partial}{\partial X_{L}}\left(\left(T_{K L}+t_{K L}^{i}\right) \frac{\partial u_{I}}{\partial X_{K}}+T_{I L}+t_{I L}^{i}\right) & =\rho^{i} \frac{\partial^{2} u_{I}}{\partial t^{2}} \Rightarrow \\
\frac{\partial}{\partial X_{L}}\left(T_{K L} \frac{\partial u_{I}}{\partial X_{K}}+t_{K L}^{i} \frac{\partial u_{I}}{\partial X_{K}}+T_{I L}+t_{I L}^{i}\right) & =\rho^{i} \frac{\partial^{2} u_{I}}{\partial t^{2}} \Rightarrow \\
\frac{\partial}{\partial X_{L}}\left(t_{K L}^{i} \frac{\partial u_{I}}{\partial X_{K}}+T_{I L}+t_{I L}^{i}\right) & =\rho^{i} \frac{\partial^{2} u_{I}}{\partial t^{2}},
\end{aligned}
$$

where the term containing the incremental stress times the incremental displacement is neglected again. Subtracting (3.11b) from (3.16) results in the 
equation of motion for the incremental displacement in initial frame of reference, as follows

$$
t_{K L}^{i} \frac{\partial^{2} u_{I}}{\partial X_{L} \partial X_{K}}+\frac{\partial T_{I L}}{\partial X_{L}}=\rho^{i} \frac{\partial^{2} u_{I}}{\partial t^{2}}
$$

The initial stress goes out of the derivative because (3.11b) claims that it is constant relative to the initial coordinate.

Equations (3.15) and (3.17) rules the motion of an incremental displacement in a pre-stressed body. In order to arrive to wave-like equations one needs to use the constitutive relationship. This is done below.

\subsection{2}

\section{Incremental constitutive and wave equations}

The Lagrangian strain is related to the Lagrangian referential; in this notation, the natural one. There are thus the Initial and Final Lagrangian strains, both related to the natural states. They are defined as [95] :

$$
\begin{aligned}
& E_{\alpha \beta}^{i}=\frac{1}{2}\left(\frac{\partial u_{\alpha}^{i}}{\partial \xi_{\beta}}+\frac{\partial u_{\beta}^{i}}{\partial \xi_{\alpha}}+\frac{\partial u_{\lambda}^{i}}{\partial \xi_{\alpha}} \frac{\partial u_{\lambda}^{i}}{\partial \xi_{\beta}}\right) \\
& E_{\alpha \beta}^{f}=\frac{1}{2}\left(\frac{\partial u_{\alpha}^{f}}{\partial \xi_{\beta}}+\frac{\partial u_{\beta}^{f}}{\partial \xi_{\alpha}}+\frac{\partial u_{\lambda}^{f}}{\partial \xi_{\alpha}} \frac{\partial u_{\lambda}^{f}}{\partial \xi_{\beta}}\right)
\end{aligned}
$$

The difference between the final and initial Lagrangian strains is the incremental strain. Considering (3.1c), it can be written as

$$
\begin{aligned}
E_{\alpha \beta} & =E_{\alpha \beta}^{f}-E_{\alpha \beta}^{i} \\
& =\frac{1}{2}\left[\frac{\partial u_{\alpha}^{f}}{\partial \xi_{\beta}}+\frac{\partial u_{\beta}^{f}}{\partial \xi_{\alpha}}+\frac{\partial u_{\lambda}^{f}}{\partial \xi_{\alpha}} \frac{\partial u_{\lambda}^{f}}{\partial \xi_{\beta}}-\left(\frac{\partial u_{\alpha}^{i}}{\partial \xi_{\beta}}+\frac{\partial u_{\beta}^{i}}{\partial \xi_{\alpha}}+\frac{\partial u_{\lambda}^{i}}{\partial \xi_{\alpha}} \frac{\partial u_{\lambda}^{i}}{\partial \xi_{\beta}}\right)\right] \\
& =\frac{1}{2}\left[\frac{\partial u_{\alpha}^{f}}{\partial \xi_{\beta}}+\frac{\partial u_{\beta}^{f}}{\partial \xi_{\alpha}}+\frac{\partial u_{\lambda}^{f}}{\partial \xi_{\alpha}} \frac{\partial u_{\lambda}^{f}}{\partial \xi_{\beta}}-\frac{\partial u_{\alpha}^{i}}{\partial \xi_{\beta}}-\frac{\partial u_{\beta}^{i}}{\partial \xi_{\alpha}}-\frac{\partial u_{\lambda}^{i}}{\partial \xi_{\alpha}} \frac{\partial u_{\lambda}^{i}}{\partial \xi_{\beta}}\right] \\
& =\frac{1}{2}\left[\frac{\partial\left(u_{\alpha}^{f}-u_{\alpha}^{i}\right)}{\partial \xi_{\beta}}+\frac{\partial\left(u_{\beta}^{f}-u_{\beta}^{i}\right)}{\partial \xi_{\alpha}}+\frac{\partial u_{\lambda}^{f}}{\partial \xi_{\alpha}} \frac{\partial u_{\lambda}^{f}}{\partial \xi_{\beta}}-\frac{\partial u_{\lambda}^{i}}{\partial \xi_{\alpha}} \frac{\partial u_{\lambda}^{i}}{\partial \xi_{\beta}}\right] \\
& =\frac{1}{2}\left[\frac{\partial u_{\alpha}}{\partial \xi_{\beta}}+\frac{\partial u_{\beta}}{\partial \xi_{\alpha}}+\frac{\partial\left(u_{\lambda}^{i}+u_{\lambda}\right)}{\partial \xi_{\alpha}} \frac{\partial\left(u_{\lambda}^{i}+u_{\lambda}\right)}{\partial \xi_{\beta}}-\frac{\partial u_{\lambda}^{i}}{\partial \xi_{\alpha}} \frac{\partial u_{\lambda}^{i}}{\partial \xi_{\beta}}\right] \\
& =\frac{1}{2}\left[\frac{\partial u_{\alpha}}{\partial \xi_{\beta}}+\frac{\partial u_{\beta}}{\partial \xi_{\alpha}}+\left(\frac{\partial u_{\lambda}^{i}}{\partial \xi_{\alpha}}+\frac{\partial u_{\lambda}}{\partial \xi_{\alpha}}\right)\left(\frac{\partial u_{\lambda}^{i}}{\partial \xi_{\beta}}+\frac{\partial u_{\lambda}}{\partial \xi_{\beta}}\right)-\frac{\partial u_{\lambda}^{i}}{\partial \xi_{\alpha}} \frac{\partial u_{\lambda}^{i}}{\partial \xi_{\beta}}\right] \\
& =\frac{1}{2}\left[\frac{\partial u_{\alpha}}{\partial \xi_{\beta}}+\frac{\partial u_{\beta}}{\partial \xi_{\alpha}}+\frac{\partial u_{\lambda}^{i}}{\partial \xi_{\alpha}} \frac{\partial u_{\lambda}^{i}}{\partial \xi_{\beta}}+\frac{\partial u_{\lambda}^{i}}{\partial \xi_{\alpha}} \frac{\partial u_{\lambda}}{\partial \xi_{\beta}}+\frac{\partial u_{\lambda}}{\partial \xi_{\alpha}} \frac{\partial u_{\lambda}^{i}}{\partial \xi_{\beta}}+\frac{\partial u_{\lambda}}{\partial \xi_{\alpha}} \frac{\partial u_{\lambda}}{\partial \xi_{\beta}}-\frac{\partial u_{\lambda}^{i}}{\partial \xi_{\alpha}} \frac{\partial u_{\lambda}^{i}}{\partial \xi_{\beta}}\right] \\
& =\frac{1}{2}\left[\frac{\partial u_{\alpha}}{\partial \xi_{\beta}}+\frac{\partial u_{\beta}}{\partial \xi_{\alpha}}+\frac{\partial u_{\lambda}^{i}}{\partial \xi_{\alpha}} \frac{\partial u_{\lambda}}{\partial \xi_{\beta}}+\frac{\partial u_{\lambda}}{\partial \xi_{\alpha}} \frac{\partial u_{\lambda}^{i}}{\partial \xi_{\beta}}+\frac{\partial u_{\lambda}}{\partial \xi_{\alpha}} \frac{\partial u_{\lambda}}{\partial \xi_{\beta}}\right] \\
& \approx \frac{1}{2}\left[\frac{\partial u_{\alpha}}{\partial \xi_{\beta}}+\frac{\partial u_{\beta}}{\partial \xi_{\alpha}}+\frac{\partial u_{\lambda}^{i}}{\partial \xi_{\alpha}} \frac{\partial u_{\lambda}}{\partial \xi_{\beta}}+\frac{\partial u_{\lambda}}{\partial \xi_{\alpha}} \frac{\partial u_{\lambda}^{i}}{\partial \xi_{\beta}}\right] \quad,
\end{aligned}
$$


where the double incremental derivative (the last term of the last but one equality) is neglected because it is supposed the wave motion produces a small perturbation.

The second Piola-Kirchhoff stress tensors are given by (3.5). The incremental stress is defined in (3.9) to be the difference of the final stress to the initial one; using these equations and (3.19) leads to the following expression for the incremental stress.

$$
\begin{aligned}
T_{\alpha \beta} & =s_{\alpha \beta}^{f}-s_{\alpha \beta}^{i}=\left[C_{\alpha \beta \gamma \delta} E_{\gamma \delta}^{f}+\frac{1}{2} C_{\alpha \beta \gamma \delta \zeta \eta} E_{\gamma \delta}^{f} E_{\zeta \eta}^{f}\right]-\left[C_{\alpha \beta \gamma \delta} E_{\gamma \delta}^{i}+\frac{1}{2} C_{\alpha \beta \gamma \delta \zeta \eta} E_{\gamma \delta}^{i} E_{\zeta \eta}^{i}\right] \\
& =C_{\alpha \beta \gamma \delta}\left(E_{\gamma \delta}^{f}-E_{\gamma \delta}^{i}\right)+\frac{1}{2} C_{\alpha \beta \gamma \delta \zeta \eta}\left(E_{\gamma \delta}^{f} E_{\zeta \eta}^{f}-E_{\gamma \delta}^{i} E_{\zeta \eta}^{i}\right) \\
& =C_{\alpha \beta \gamma \delta} E_{\gamma \delta}+\frac{1}{2} C_{\alpha \beta \gamma \delta \zeta \eta}\left[\left(E_{\gamma \delta}^{i}+E_{\gamma \delta}\right)\left(E_{\zeta \eta}^{i}+E_{\zeta \eta}\right)-E_{\gamma \delta}^{i} E_{\zeta \eta}^{i}\right] \\
& =C_{\alpha \beta \gamma \delta} E_{\gamma \delta}+\frac{1}{2} C_{\alpha \beta \gamma \delta \zeta \eta}\left[E_{\gamma \delta}^{i} E_{\zeta \eta}^{i}+E_{\gamma \delta} E_{\zeta \eta}^{i}+E_{\gamma \delta}^{i} E_{\zeta \eta}+E_{\gamma \delta} E_{\zeta \eta}-E_{\gamma \delta}^{i} E_{\zeta \eta}^{i}\right] \\
& =C_{\alpha \beta \gamma \delta} E_{\gamma \delta}+\frac{1}{2} C_{\alpha \beta \gamma \delta \zeta \eta}\left[E_{\gamma \delta} E_{\zeta \eta}^{i}+E_{\gamma \delta}^{i} E_{\zeta \eta}+E_{\gamma \delta} E_{\zeta \eta}\right] \\
& =C_{\alpha \beta \gamma \delta} E_{\gamma \delta}+C_{\alpha \beta \gamma \delta \zeta \eta}\left[E_{\gamma \delta}^{i} E_{\zeta \eta}+\frac{1}{2} E_{\gamma \delta} E_{\zeta \eta}\right]
\end{aligned}
$$

where in the last row the symmetry of the sixth-order stiffness tensor $C_{\alpha \beta \gamma \delta \zeta \eta}=$ $C_{\alpha \beta \zeta \eta \gamma \delta}$ is used (the symmetries on stiffness tensor are demonstrated in Appendix A.1.2, this particular one is found in (A.14b)). The last term can be neglected because contains the product of two incremental entities, resulting in the simpler form:

$$
T_{\alpha \beta}=C_{\alpha \beta \gamma \delta} E_{\gamma \delta}+C_{\alpha \beta \gamma \delta \zeta \eta} E_{\gamma \delta}^{i} E_{\zeta \eta}
$$

Further considering that the product of initial strain and incremental strain can be approximated by their linear parts, the relationship below is finally obtained:

$$
T_{\alpha \beta}=C_{\alpha \beta \gamma \delta} E_{\gamma \delta}+C_{\alpha \beta \gamma \delta \zeta \eta} \varepsilon_{\gamma \delta} e_{\zeta \eta}
$$

in which $\varepsilon$ and $\mathbf{e}$ are the linear part of the initial and incremental strains, respectively, and given by

$$
\begin{aligned}
& \varepsilon_{\alpha \beta}=\frac{1}{2}\left(\frac{\partial u_{\alpha}^{i}}{\partial \xi_{\beta}}+\frac{\partial u_{\beta}^{i}}{\partial \xi_{\alpha}}\right), \\
& e_{\alpha \beta}=\frac{1}{2}\left(\frac{\partial u_{\alpha}}{\partial \xi_{\beta}}+\frac{\partial u_{\beta}}{\partial \xi_{\alpha}}\right) .
\end{aligned}
$$

The constitutive relationship between the initial stress and initial strain is the linear usual one given by the infinitesimal elasticity, and stated in (2.3). In the current notation this is written as

$$
s_{\alpha \beta}^{i}=C_{\alpha \beta \gamma \delta} \varepsilon_{\gamma \delta}
$$


Developing (3.22) by substituting (3.19) and (3.23b) in it we get

$$
\begin{aligned}
T_{\alpha \beta} & =C_{\alpha \beta \gamma \delta} \frac{1}{2}\left[\frac{\partial u_{\gamma}}{\partial \xi_{\delta}}+\frac{\partial u_{\delta}}{\partial \xi \gamma}+\frac{\partial u_{\lambda}^{i}}{\partial \xi_{\gamma}} \frac{\partial u_{\lambda}}{\partial \xi_{\delta}}+\frac{\partial u_{\lambda}}{\partial \xi_{\gamma}} \frac{\partial u_{\lambda}^{i}}{\partial \xi_{\delta}}\right] \\
& +C_{\alpha \beta \gamma \delta \zeta \eta} \varepsilon_{\gamma \delta} \frac{1}{2}\left(\frac{\partial u_{\zeta}}{\partial \xi_{\eta}}+\frac{\partial u_{\eta}}{\partial \xi_{\zeta}}\right),
\end{aligned}
$$

considering the symmetries $C_{\alpha \beta \gamma \delta}=C_{\alpha \beta \delta \gamma}$ and $C_{\alpha \beta \gamma \delta \zeta \eta}=C_{\alpha \beta \gamma \delta \eta \zeta}$, (3.25) simplifies to

$$
T_{\alpha \beta}=C_{\alpha \beta \gamma \delta}\left(\frac{\partial u_{\gamma}}{\partial \xi_{\delta}}+\frac{\partial u_{\lambda}^{i}}{\partial \xi_{\gamma}} \frac{\partial u_{\lambda}}{\partial \xi_{\delta}}\right)+C_{\alpha \beta \gamma \delta \zeta \eta} \varepsilon_{\gamma \delta} \frac{\partial u_{\zeta}}{\partial \xi_{\eta}}
$$

further considering the symmetry $C_{\alpha \beta \gamma \delta \zeta \eta}=C_{\alpha \beta \zeta \eta \gamma \delta}$, (3.26) results in the incremental stress-strain relationship in the natural frame of reference, which is given by

where the tensor $\mathbf{B}^{n}$ is

$$
T_{\alpha \beta}=B_{\alpha \beta \gamma \delta}^{n} \frac{\partial u_{\gamma}}{\partial \xi_{\delta}}
$$

$$
B_{\alpha \beta \gamma \delta}^{n}=C_{\alpha \beta \gamma \delta}+C_{\alpha \beta \lambda \delta} \frac{\partial u_{\gamma}^{i}}{\partial \xi_{\lambda}}+C_{\alpha \beta \lambda \delta \zeta \eta} \varepsilon_{\zeta \eta}
$$

Applying the constitutive relationship (3.27) into the equation of motion (3.15), where the original index $\gamma$ was replaced by $\mu$, imply in

$$
\begin{aligned}
\frac{\partial}{\partial \xi_{\beta}}\left[s_{\alpha \beta}^{i} \frac{\partial u_{\mu}}{\partial \xi_{\alpha}}+B_{\alpha \beta \gamma \delta}^{n} \frac{\partial u_{\gamma}}{\partial \xi_{\delta}} \frac{\partial u_{\mu}^{i}}{\partial \xi_{\alpha}}+B_{\mu \beta \gamma \delta}^{n} \frac{\partial u_{\gamma}}{\partial \xi_{\delta}}\right] & =\rho^{0} \frac{\partial^{2} u_{\mu}}{\partial t^{2}} \Rightarrow \\
\frac{\partial}{\partial \xi_{\beta}}\left[s_{\alpha \beta}^{i} \frac{\partial u_{\mu}}{\partial \xi_{\alpha}}+\left(B_{\alpha \beta \gamma \delta}^{n} \frac{\partial u_{\mu}^{i}}{\partial \xi_{\alpha}}+B_{\mu \beta \gamma \delta}^{n}\right) \frac{\partial u_{\gamma}}{\partial \xi_{\delta}}\right] & =\rho^{0} \frac{\partial^{2} u_{\mu}}{\partial t^{2}},
\end{aligned}
$$

which introduces a new tensor, namely,

$$
\Gamma_{\mu \beta \gamma \delta}=B_{\alpha \beta \gamma \delta}^{n} \frac{\partial u_{\mu}^{i}}{\partial \xi_{\alpha}}+B_{\mu \beta \gamma \delta}^{n} .
$$

Developing the tensor $\mathbf{B}^{n}$ and neglecting second order term of the initial strain, results in

$$
\Gamma_{\alpha \beta \gamma \delta}=C_{\alpha \beta \gamma \delta}+C_{\alpha \beta \lambda \delta} \frac{\partial u_{\gamma}^{i}}{\partial \xi_{\lambda}}+C_{\lambda \beta \gamma \delta} \frac{\partial u_{\alpha}^{i}}{\partial \xi_{\lambda}}+C_{\alpha \beta \lambda \delta \zeta \eta} \varepsilon_{\zeta \eta}
$$

Noticing that in (3.29) the index $\alpha$ in the first term is isolated from the second term and then can be replaced, (3.29) becomes

$$
\frac{\partial}{\partial \xi_{\beta}}\left[s_{\delta \beta}^{i} \frac{\partial u_{\mu}}{\partial \xi_{\delta}}+\Gamma_{\mu \beta \gamma \delta} \frac{\partial u_{\gamma}}{\partial \xi_{\delta}}\right]=\rho^{0} \frac{\partial^{2} u_{\mu}}{\partial t^{2}}
$$

as the tensor $s_{\delta \beta}^{i}$ is symmetric 


$$
\begin{aligned}
\frac{\partial}{\partial \xi_{\beta}}\left[s_{\beta \delta}^{i} \frac{\partial u_{\mu}}{\partial \xi_{\delta}}+\Gamma_{\mu \beta \gamma \delta} \frac{\partial u_{\gamma}}{\partial \xi_{\delta}}\right] & =\rho^{0} \frac{\partial^{2} u_{\mu}}{\partial t^{2}} \Rightarrow \\
\frac{\partial}{\partial \xi_{\beta}}\left[s_{\beta \delta}^{i} \delta_{\mu \gamma} \frac{\partial u_{\gamma}}{\partial \xi_{\delta}}+\Gamma_{\mu \beta \gamma \delta} \frac{\partial u_{\gamma}}{\partial \xi_{\delta}}\right] & =\rho^{0} \frac{\partial^{2} u_{\mu}}{\partial t^{2}} \Rightarrow \\
\frac{\partial}{\partial \xi_{\beta}}\left[\left(s_{\beta \delta}^{i} \delta_{\mu \gamma}+\Gamma_{\mu \beta \gamma \delta}\right) \frac{\partial u_{\gamma}}{\partial \xi_{\delta}}\right] & =\rho^{0} \frac{\partial^{2} u_{\mu}}{\partial t^{2}} .
\end{aligned}
$$

The parenthesis in the equation above is defined as the tensor

$$
A_{\mu \beta \gamma \delta}^{n}=s_{\beta \delta}^{i} \delta_{\mu \gamma}+\Gamma_{\mu \beta \gamma \delta}
$$

adding the constitutive equation for the initial stress $s_{\alpha \beta}^{i}$ declared in (3.24) into $(3.34)$

$$
A_{\mu \beta \gamma \delta}^{n}=C_{\beta \delta \zeta \eta} \varepsilon_{\zeta \eta} \delta_{\mu \gamma}+\Gamma_{\mu \beta \gamma \delta}
$$

or, renaming indexes,

$$
A_{\alpha \beta \gamma \delta}^{n}=C_{\beta \delta \zeta \eta} \varepsilon_{\zeta \eta} \delta_{\alpha \gamma}+\Gamma_{\alpha \beta \gamma \delta}
$$

For an homogenous medium where either the initial stress and the first initial displacement derivative $\left(\frac{\partial u_{\alpha}^{i}}{\partial \xi_{\beta}}\right)$ are constant throughout the medium, a typical wave equation is reached

$$
A_{\alpha \beta \gamma \delta}^{n} \frac{\partial^{2} u_{\gamma}}{\partial \xi_{\beta} \partial \xi_{\delta}}=\rho^{0} \frac{\partial^{2} u_{\alpha}}{\partial t^{2}}
$$

This last equation is the wave equation for a pre-stressed body in the natural frame of reference. The tensor $\mathbf{A}^{n}$ plays the role of the fourth order stiffness tensor in an unstressed medium. It is worth to note that differently from the unstressed medium the constitutive equation and the wave equation do not share the same tensor. Incremental stress-strain are ruled by the tensor $B^{n}$ by means of equation (3.27) whereas the wave relation by the tensor $A^{n}$.

Similar development leads to the constitutive equation and wave equation in the initial frame of reference. The incremental stress is obtained calculating (3.10) by means (3.8d) and (3.8a), then it is used in (3.17) in order to obtain the wave equation. The incremental stress-strain relationship in the initial frame of reference is given by

$$
T_{I J}=B_{I J K L}^{i} \frac{\partial u_{K}}{\partial X_{L}}
$$

where the tensor $\mathbf{B}^{i}$ is

$$
\begin{aligned}
B_{I J K L}^{i} & =C_{I J K L}+C_{M J K L} \frac{\partial u_{I}^{i}}{\partial X_{M}}+C_{I M K L} \frac{\partial u_{J}^{i}}{\partial X_{M}}+C_{I J M L} \frac{\partial u_{K}^{i}}{\partial X_{M}}+C_{I J K M} \frac{\partial u_{L}^{i}}{\partial X_{M}} \\
& -C_{I J K L} \varepsilon_{N N}+C_{I J K L M N} \varepsilon_{M N} .
\end{aligned}
$$

With the same homogeneity condition, the wave equation for the initial frame of reference is reached: 


$$
A_{I J K L}^{i} \frac{\partial^{2} u_{K}}{\partial X_{J} \partial X_{L}}=\rho^{0} \frac{\partial^{2} u_{I}}{\partial t^{2}}
$$

where the tensor $\mathbf{A}^{i}$ is

$$
A_{I J K L}^{i}=C_{J L M N} \varepsilon_{M N} \delta_{I K}+C_{I J K L} \varepsilon_{N N}+B_{I J K L}^{i}
$$

and the following relationship between the density in natural and initial frames was used:

$$
\frac{\rho^{i}}{\rho^{0}}=1-\varepsilon_{i i}
$$

\section{2}

\section{Particular case of null rotational on strain}

As done by Gandhi et al. [34,96] it will be assumed that the rotation term on the strain is null, i.e.,

$$
r_{\alpha \beta}=\frac{1}{2}\left(\frac{\partial u_{\alpha}^{i}}{\partial \xi_{\beta}}-\frac{\partial u_{\beta}^{i}}{\partial \xi_{\alpha}}\right)=0
$$

implying that

$$
\varepsilon_{\alpha \beta}=\frac{\partial u_{\alpha}^{i}}{\partial \xi_{\beta}}
$$

and thus the tensors can be simplified from (3.36) to

$$
A_{\alpha \beta \gamma \delta}^{n}=C_{\beta \delta \zeta \eta} \varepsilon_{\zeta \eta} \delta_{\alpha \gamma}+C_{\alpha \beta \gamma \delta}+C_{\alpha \beta \lambda \delta} \varepsilon_{\gamma \lambda}+C_{\lambda \beta \gamma \delta} \varepsilon_{\alpha \lambda}+C_{\alpha \beta \lambda \delta \zeta \eta} \varepsilon_{\zeta \eta}
$$

and from (3.28) to

$$
B_{\alpha \beta \gamma \delta}^{n}=C_{\alpha \beta \gamma \delta}+C_{\alpha \beta \lambda \delta} \varepsilon_{\gamma \lambda}+C_{\alpha \beta \lambda \delta \zeta \eta} \varepsilon_{\zeta \eta}
$$

In order to simplify the tensor at the initial frame one needs to relate $\frac{\partial u_{I}^{i}}{\partial X_{J}}$ to $\varepsilon_{I J}$. It is convenient to use the Gradient of Deformation defined in (3.7)). Developing this by applying (3.1a) into it leads to

$$
F_{I J}=\frac{\partial\left(u_{I}^{i}+\xi_{I}\right)}{\partial \xi_{J}}=\frac{\partial u_{I}^{i}}{\partial \xi_{J}}+\frac{\partial \xi_{I}}{\partial \xi_{J}}=\frac{\partial u_{I}^{i}}{\partial \xi_{J}}+\delta_{I J},
$$

and in the particular case of (3.44),

$$
F_{I J}=\varepsilon_{I J}+\delta_{I J}
$$

Expanding now $\frac{\partial u_{I}^{i}}{\partial X_{J}}$ with (3.1a) one gets

$$
\frac{\partial u_{I}^{i}}{\partial X_{J}}=\frac{\partial\left(X_{I}-\xi_{I}\right)}{\partial X_{J}}=\delta_{I J}-\frac{\partial \xi_{I}}{\partial X_{J}}
$$

where by definition (3.7) the last term is the inverse of the Gradient of Deformation, and thus

$$
\frac{\partial u_{I}^{i}}{\partial X_{J}}=\delta_{I J}-F_{I J}^{-1},
$$

in this special case one reach the relationship 


$$
\frac{\partial u_{I}^{i}}{\partial X_{J}}=\delta_{I J}-\left(\varepsilon_{I J}+\delta_{I J}\right)^{-1}
$$

This last equation means that the terms necessary for calculating the acoustoelastic tensor $B^{i}$, and then $A^{i}$, at the initial frame of reference can be obtained from the conventional initial infinitesimal Lagrangian strain.

\section{3}

\section{Tensor symmetries}

The acoustoelastic tensors shown above do not present a priori the same full symmetry condition as the second order stiffness tensor, given by (2.4). For a naturally anisotropic medium the symmetries for the acoustoelastic tensors at the natural frame of reference are

$$
\begin{aligned}
& A_{\alpha \beta \gamma \delta}^{n}=A_{\gamma \delta \alpha \beta}^{n} \quad, \text { and in general } \\
& A_{\alpha \beta \gamma \delta}^{n} \neq A_{\beta \alpha \gamma \delta}^{n} \neq A_{\alpha \beta \delta \gamma}^{n}, \\
& B_{\alpha \beta \gamma \delta}^{n}=B_{\beta \alpha \gamma \delta}^{n}, \text {, and in general } \\
& B_{\alpha \beta \gamma \delta}^{n} \neq B_{\alpha \beta \delta \gamma}^{n} \neq B_{\gamma \delta \alpha \beta}^{n},
\end{aligned}
$$

at the initial frame

$$
\begin{aligned}
& A_{I J K L}^{i}=A_{K L I J}^{i} \quad, \text { and in general } \\
& A_{I J K L}^{i} \neq A_{J I K L}^{i} \neq A_{I J L K}^{i}, \\
& B_{I J K L}^{i}=B_{J I K L}^{i}=B_{I J L K}^{i}=B_{K L I J}^{i} .
\end{aligned}
$$

As it can be seen, just the tensor $\mathbf{B}^{i}$ presents the full symmetry; the symmetry condition for the $\mathbf{A}^{n}$ and $\mathbf{A}^{i}$ are the same.

In order to understand the physical meaning of this lack of symmetry it is convenient to first investigate the initial frame of reference. The initial stress has two effects that can be a source of asymmetry, namely constitutive and motion effects.

The constitutive effect is the change in the incremental stress-strain relationship. This if manifested by the $\mathbf{B}^{i}$ tensor that becomes different from the usual stiffness tensor $\mathbf{C}$, which holds for the unloaded medium, see (3.39). Since by addressing the problem at the initial frame, the motion and constitutive phenomena take place at the same state as the reference does, i.e. the initial one, there is no reason for this tensor to present any lack of symmetry; $\mathbf{B}^{i}$ present the full symmetry condition (3.53b). In other words, the initial stress just acts as an additional stiffener, it can even causes anisotropy to the material, but this tensor still has the physical meaning of an constitutive 
tensor for the incremental stress-strain related to this very state; it thus does not lose any of the symmetries that any material must present.

The motion effect comes from the motion equation (3.12b), which must be expressed in terms of the final stress, due to the Newton's second law (i.e. the net force must be used in the motion equation). This equation was developed up to (3.17), where it is clear that the initial stress is present (first term of the left side), as well as the incremental one (last term of the left side). The incremental stress is replaced by the constitutive incremental equation (3.38), in order to express the equation by means of the incremental displacement. Then, in order to reach a wave-like equation, the initial stress term (first term in 3.17) is added to the tensor $\mathbf{B}^{i}$ resulting in a new tensor $\mathbf{A}^{i}$. This tensor, however, has no physical meaning of an stiffness tensor. It is just the sum of the new stiffness tensor with the initial stress. All the lack of symmetry in $\mathbf{A}^{i}$ comes from the motion effect; the symmetries presented in $\mathbf{A}^{i}$ are the same as those encountered in the initial stress term of 3.17.

At the natural frame, the interpretation is less straightforward. The motion effect is still a reason for the lack of symmetry. The first term in (3.15) plays the role of the first term in (3.17) for the initial case. However there is additionally the second term (3.15) that is not present in (3.17). Moreover, the constitutive effect at this frame results in additional lack of symmetries in $\mathbf{B}^{n}$. These asymmetries are consequences of relating the physical phenomena of wave motion and incremental constitutive relationship to a state different from the reference one (the phenomena take place at the initial frame, whereas the reference frame is the natural). In other words, in order to embed the statical initial deformation in the resulting velocity, additional terms arise which force the tensors, both $\mathbf{B}^{n}$ and $\mathbf{A}^{n}$, to not comply with the usual symmetries.

There is a particular case in which the tensor $\mathbf{A}^{n}$ presents the following additional symmetry:

$$
\begin{aligned}
& A_{\alpha \beta \gamma \delta}^{n}=A_{\gamma \delta \alpha \beta}^{n} \quad, \\
& A_{\alpha \beta \gamma \delta}^{n}=A_{\beta \alpha \delta \gamma}^{n} \quad, \text { but in general } \\
& A_{\alpha \beta \gamma \delta}^{n} \neq A_{\beta \alpha \gamma \delta}^{n} \neq A_{\alpha \beta \delta \gamma}^{n} .
\end{aligned}
$$

This can be understood as if the motion and the constitutive effects mutually compensate each other, ensuring less loss of symmetry. This only happens at the natural frame because at the initial frame the unique source of asymmetry is the motion effect (first term in 3.17). Thus, there is no other term that could possibly help in compensating the lack of symmetry of $\mathbf{A}^{i}$. This can be an advantage as noted Duquennoy et al [29]. All the symmetries stated in this section are prooved in Appendix A. 
A comprehensive analysis of anisotropy induced by the load is carried out in Fuck and Tsvankin [97]. This study is however restricted to the assumption in which the tensor $\mathbf{A}$ is composed just by the sum of the fourth and sixth order stiffness tensors, i.e. the first and last terms of (3.31) or (3.39) only. In this case the tensor must present the full symmetries. In other words, under this assumption load can cause anisotropy but does not cause loss of the basic symmetries.

\subsection{1}

\section{Numerical example}

In order to allow visualization of the above symmetries it is convenient to report a numerical example. These are fourth order tensor, thus writing then in two dimension paper sheet is not possible, it is necessary some artifice. It is used here the extended Voigt notation that consists of writing a fourth order tensor, with a total of $81(=3 \times 3 \times 3 \times 3)$ entries in a $9 \times 9$ matrix. In this notation $m$ represent the two indexes $i j$ together and $n$ represents $k l$, by the following rule in Table 3.1.

Table 3.1: Extended Voigt notation.

\begin{tabular}{c|c}
$i j$ or $k l$ & $m$ or $n$ \\
\hline 11 & 1 \\
22 & 2 \\
33 & 3 \\
23 & 4 \\
13 & 5 \\
12 & 6 \\
32 & 7 \\
31 & 8 \\
21 & 9
\end{tabular}

The numerical example presented here stands for a naturally isotropic aluminum material subjected to a tensile stress of $\sigma_{11}=120 \mathrm{MPa}$ with the constants elastic and acoustoelastic constants presented in table 3.2.

Table 3.2: Aluminum constants [40].

\begin{tabular}{cccccc}
\hline$\rho$ & $\lambda$ & $\mu$ & $l$ & $m$ & $n$ \\
$\mathrm{~kg} / \mathrm{m}^{3}$ & $\mathrm{GPa}$ & $\mathrm{GPa}$ & $\mathrm{GPa}$ & $\mathrm{GPa}$ & $\mathrm{GPa}$ \\
\hline 2700 & 54.9 & 26.5 & -252.2 & -324.9 & -351.2 \\
\hline
\end{tabular}

The tensors $\mathbf{A}^{\mathbf{n}}$ and $\mathbf{A}^{\mathbf{i}}$ assume the values in (3.55) and (3.56), respectively, where color was used to highlight symmetry breaks, and the row and column header refer to the $i j$ and $k l$ indexes, respectively. 


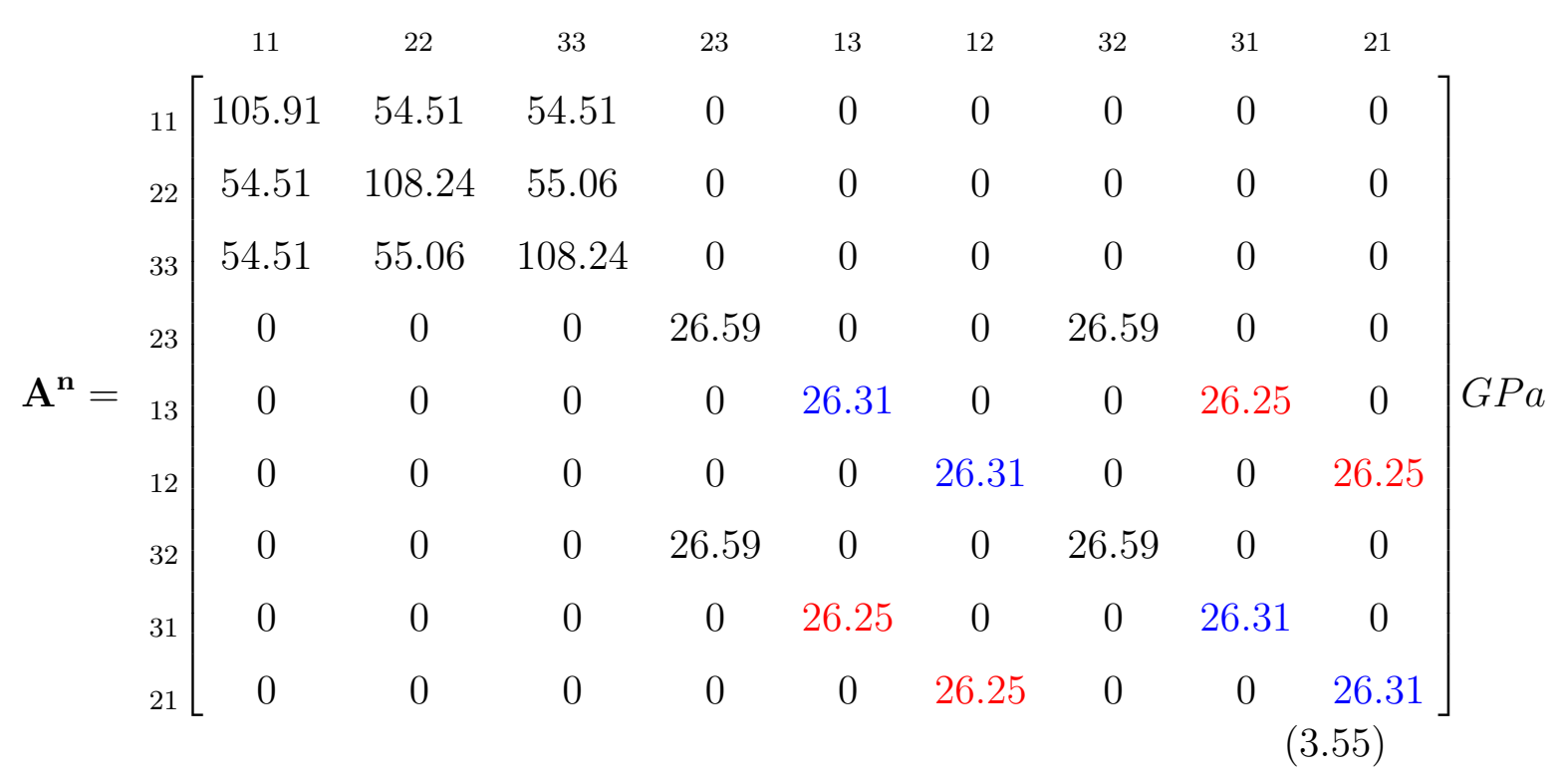

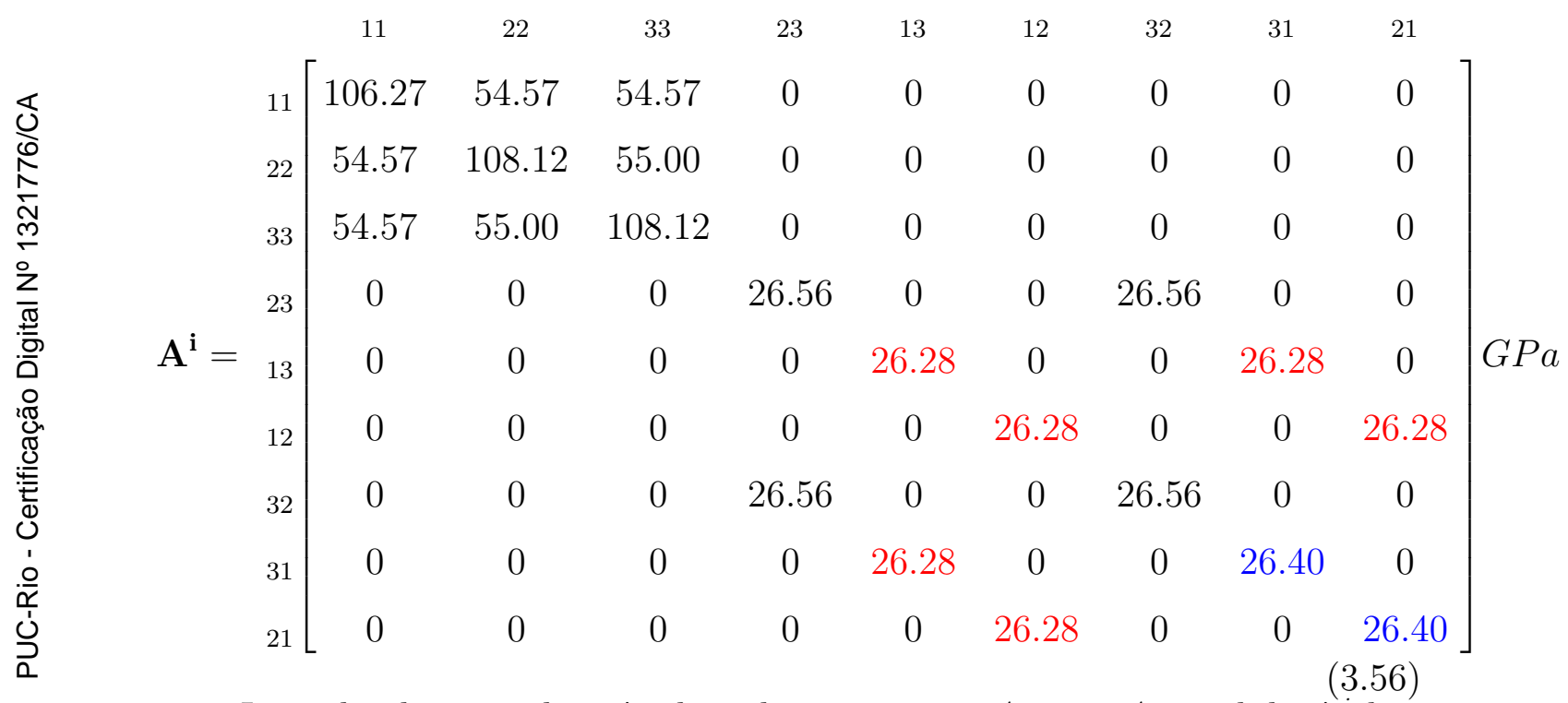

It is clearly seen that $\mathbf{A}^{n}$ has the symmetry $A_{i j k l}^{n}=A_{j i l k}^{n}$ while $\mathbf{A}^{i}$ has not.

This example also illustrates that load makes an isotropic medium to behave anisotropically. However, wave propagation in loaded body is still distinct from wave in anisotropic media by two important facts. First, the tensor $\mathbf{A}$, in both references, still miss the full set of symmetries present in anisotropic media. Second, the constitutive equation uses other tensor, B. In the infinitesimal elastic theory both wave motion and stress-strain relationship share the same stiffness tensor, regardless the natural anisotropy it may present. 


\section{4}

\section{Relationship between velocity at initial and natural frames for bulk waves}

The velocities at the natural and initial frame of reference for a bulk wave can be related by $[27,91,98]$

$$
c^{i}=c^{n}+c^{0} \varepsilon_{i}
$$

where $c^{n}$ is the bulk wave velocity of interest (with the specified polarization) in the natural frame of reference, $c^{0}$ is the corresponding velocity for the null stress condition, $c^{i}$ is the bulk wave velocity of interest in the initial frame of reference, and $\varepsilon_{i}$ is the principal initial infinitesimal strain in the direction of the wave propagation. The difference between the velocities is then

$$
c^{i}-c^{n}=c^{0} \varepsilon_{i}
$$

For a wave propagating in $\xi_{1}$ direction (3.58) becomes

$$
c^{i}-c^{n}=c^{0} \varepsilon_{11}
$$

and propagating along the $\xi_{3}$ direction is written as

$$
c^{i}-c^{n}=c^{0} \varepsilon_{33} .
$$

These relationships are used in the next chapters as an approximation for the Lamb wave velocity; its validity is also analyzed. 


\section{4}

\section{Acoustoelastic Lamb waves}

The acoustoelastic theory applied to guided waves is a relatively new branch of study. An initial attempt of addressing the acoustoelastic effect for guided waves was done by Chen and Wilcox [33] and Loveday et al. [32,99,100], where the acoustoelastic formulation was not complete because finite strain was considered but the third order elastic constants were not. The acoustoelastic effect on Lamb waves in plates has been completely developed firstly by Gandhi et al. [34], where the theoretical framework has been developed, and experimentally evaluated, for a general biaxially applied stress. That work allowed one to analytically obtain the velocity dependence on stress, by calculating the dispersion curves for each mode. Some very recent work also developed the acoustoelastic theory for Lamb waves as done by Pau and Lanza di Scalea [101] for longitudinal and transverse applied stress, and by Mohabuth et al. [102] for longitudinal applied stress only. Where in both works the acoustoelastic approach is different from Gandhi et al. but the results are coherent.

There are few experimental works specifically on measuring stress on plates using acoustoelasticity of Lamb waves. Murayama et al [103-105], used non-contact EMAT transducer in order to measure the acustoelastic effect of a tensile load, independent of geometry change, in a metallic plate for the S0 Lamb wave mode propagated at $500 \mathrm{kHz} \times \mathrm{mm}$; the acoustoelastic sensitivity were experimentally obtained and then used in order to measure stress by time-of-flight observation. Zangrai et al. [106] measured the acoustoelastic effect for longitudinal and perpendicular stress for an aluminum plate for A0 and S0 modes at frequencies from $300 \mathrm{kHz} \times \mathrm{mm}$ up to $500 \mathrm{kHz} \times \mathrm{mm}$, and also investigated the tightness assessment of bolted joints by means of acoustoelastic effect. Amjad et al. [107] determined the time-of-flight shift for A0 and S0 modes at several frequencies concluding high dependence on frequency. Shi et al. [24] used the Gandhi's theory in order to absolutely estimate biaxial stress applied in an aluminum plate using the S0 mode around $1.3 \mathrm{kHz} \times \mathrm{mm}$. In all these works the system was excited by a narrow band tone burst in a predefined central frequency, in order to allow propagation of a single Lamb mode and strain measurement was performed by time-shift observation, 
either by zero crossing or cross correlation.

It is worth to point out that Gandhi et al. [34] work is considered pioneer for the traditional acoutoelasticity within the weakly non-linear theory. Nevertheless, under the exact non-linear elasticity the propagation of waves in prestressed plates has been widely studied over the last decades, a comprehensive reference collection can be found in Kayestha et al. [108]. Great attention has been drawn to incompressible [109] and compressible [110] bodies, specially for soft materials, through many different constitutive theories. Stability and asymptotic characteristics have also been relevant concerns [111,112].

In this chapter the theory of Lamb wave propagation in a material under initial deformation is developed by applying the equations of motion developed in chapter 3 into the theory of Lamb wave propagation in anisotropic plate. It is reviewed here the work of Gandhi et al. [34], where it has been used the partial wave technique approach for the acoustoelastic Lamb wave propagation under biaxial initial stress. Then simplifications are made for the case of longitudinal stress. Special attention is given to some generalizations discovered on that work that preclude the use of this procedure for obtaining the dispersion curves in difference conditions as the ones originally used, for instance, at the initial frame of reference. These were corrected here. In the chapter's last section, the partial wave technique for anisotropic media is presented and shown to be a particular case of the general procedure for acoustoelastic waves. Then, the use of Effective Elastic Constants for an equivalent anisotropic medium is analyzed as an approximation for the full procedure. This theoretical basis is relevant in the sequel.

\section{1}

\section{Partial wave technique for acoustoelastic Lamb wave propagation}

In order to obtain the dispersion curves, it is used the partial wave technique. The curves can be referred to the natural or initial frame of reference. All the following development can be expressed in both frames of reference, defined in the last chapter. It is used lower case roman letters for the coordinates, i.e., $\mathbf{x}$ may be equal to $\xi$ or $\mathbf{X}$; the tensors are not specified in any frame of reference, thus $\mathbf{A}$ and $\mathbf{B}$ may be $\mathbf{A}^{n}$ and $\mathbf{B}^{n}$ or $\mathbf{A}^{i}$ and $\mathbf{B}^{i}$, respectively.

The partial wave technique assumes solution for the displacement of the form

$$
u_{m}=U_{m} e^{i \kappa\left(x_{1}+\alpha x_{3}-c t\right)},
$$

where $\kappa$ is the wavenumber in $x_{1}$ direction, $\alpha \cdot \kappa$ is the wavenumber in the $x_{3}$, and $c$ is the phase velocity. The displacement $u_{m}$ represents a partial wave, and 
its amplitude is $U_{m}$. In order to reach a Christoffel equation, (4.1) is applied into the wave equation, either (3.37) or (3.40) and rewritten here as

$$
A_{i j k l} \frac{\partial^{2}}{\partial x_{j} \partial x_{l}} u_{k}=\rho^{0} \frac{\partial^{2}}{\partial t^{2}} u_{i} .
$$

Then it can be arranged in matrix form as

$$
\begin{aligned}
\mathbf{K U} & =0 \quad \text { or } \\
K_{i k} U_{k} & =0 \quad .
\end{aligned}
$$

The derivation of this equation is carried out below. Initially, it is necessary to calculate the spatial and temporal derivatives of $u_{k}$ as follows.

$$
\begin{aligned}
\frac{\partial^{2} u_{m}}{\partial x_{j} \partial x_{l}} & =-\kappa^{2} u_{m} \delta_{1 j} \delta_{1 l}-\alpha \kappa^{2} u_{m}\left(\delta_{1 j} \delta_{3 l}+\delta_{3 j} \delta_{1 l}\right)-\alpha^{2} \kappa^{2} u_{m} \delta_{3 j} \delta_{3 l} \\
& =-\kappa^{2} u_{m}\left[\delta_{1 j} \delta_{1 l}+\alpha\left(\delta_{1 j} \delta_{3 l}+\delta_{3 j} \delta_{1 l}\right)+\alpha^{2} \delta_{3 j} \delta_{3 l}\right]
\end{aligned}
$$

and the time derivative is simply

$$
\frac{\partial^{2} u_{m}}{\partial t^{2}}=-c^{2} \kappa^{2} u_{m}
$$

Applying (4.4) and (4.5) into (4.2) we get

$$
-A_{i j k l} \kappa^{2} u_{k}\left[\delta_{1 j} \delta_{1 l}+\alpha\left(\delta_{1 j} \delta_{3 l}+\delta_{3 j} \delta_{1 l}\right)+\alpha^{2} \delta_{3 j} \delta_{3 l}\right]=-\rho c^{2} \kappa^{2} u_{i}
$$

and rearranging,

$$
\begin{aligned}
\rho c^{2} \kappa^{2} u_{i}-A_{i j k l} \kappa^{2} u_{k}\left[\delta_{1 j} \delta_{1 l}+\alpha\left(\delta_{1 j} \delta_{3 l}+\delta_{3 j} \delta_{1 l}\right)+\alpha^{2} \delta_{3 j} \delta_{3 l}\right] & =0 \Rightarrow \\
\rho c^{2} \kappa^{2} u_{k} \delta_{i k}-\kappa^{2} u_{k}\left[A_{i 1 k 1}+\alpha\left(A_{i 1 k 3}+A_{i 3 k 1}\right)+\alpha^{2} A_{i 3 k 3}\right] & =0 \Rightarrow \\
{\left[\rho c^{2} \kappa^{2} \delta_{i k}-\kappa^{2}\left[A_{i 1 k 1}+\alpha\left(A_{i 1 k 3}+A_{i 3 k 1}\right)+\alpha^{2} A_{i 3 k 3}\right]\right] u_{k} } & =0
\end{aligned} .
$$

Thus applying (4.1) into (4.7),

$$
\left[\rho c^{2} \kappa^{2} \delta_{i k}-\kappa^{2}\left[A_{i 1 k 1}+\alpha\left(A_{i 1 k 3}+A_{i 3 k 1}\right)+\alpha^{2} A_{i 3 k 3}\right]\right] U_{k} e^{i \kappa\left(x_{1}+\alpha x_{3}-c t\right)}=0,
$$

where the squared wavenumber and exponential oscillation can be eliminated, leading to (4.3) in which the coefficients of $\mathbf{K}$ are

$$
K_{i k}=\rho c^{2} \delta_{i k}-A_{i 1 k 1}-\alpha\left(A_{i 1 k 3}+A_{i 3 k 1}\right)+\alpha^{2} A_{i 3 k 3}
$$

Because $\mathbf{A}$ presents the symmetry condition $A_{i j k l}=A_{k l i j}$ in any frame of reference, stated in (3.52a) and (3.53a), it can be verified that $\mathbf{K}$ is symmetric, that is,

$$
\begin{aligned}
K_{k i} & =\rho c^{2} \delta_{k i}-A_{k 1 i 1}-\alpha\left(A_{k 1 i 3}+A_{k 3 i 1}\right)-\alpha^{2} A_{k 3 i 3} \\
& =\rho c^{2} \delta_{i k}-A_{i 1 k 1}-\alpha\left(A_{i 3 k 1}+A_{i 1 k 3}\right)-\alpha^{2} A_{i 3 k 3}=K_{i k} .
\end{aligned}
$$


Each entry is presented below

$$
\begin{aligned}
K_{11} & =\rho c^{2}-A_{1111}-\alpha\left(A_{1113}+A_{1311}\right)-\alpha^{2} A_{1313} \\
& =\rho c^{2}-A_{1111}-2 \alpha A_{1113}-\alpha^{2} A_{1313} \\
K_{22} & =\rho c^{2}-A_{2121}-\alpha\left(A_{2123}+A_{2321}\right)-\alpha^{2} A_{2323} \\
& =\rho c^{2}-A_{2121}-2 \alpha A_{2123}-\alpha^{2} A_{2323} \\
K_{33} & =\rho c^{2}-A_{3131}-\alpha\left(A_{3133}+A_{3331}\right)-\alpha^{2} A_{3333} \\
& =\rho c^{2}-A_{3131}-2 \alpha A_{3133}-\alpha^{2} A_{3333} \\
K_{12}=K_{21} & =-A_{1121}-\alpha\left(A_{1123}+A_{1321}\right)-\alpha^{2} A_{1323} \\
K_{13}=K_{31} & =-A_{1131}-\alpha\left(A_{1133}+A_{1331}\right)-\alpha^{2} A_{1333} \\
K_{23}=K_{32} & =-A_{2131}-\alpha\left(A_{2133}+A_{2331}\right)-\alpha^{2} A_{2333} .
\end{aligned}
$$

For the particular case of a naturally isotropic material submitted to biaxially stress on the plane $x_{3}=$ constant (or plane 1-2), the material shows this plane as a plane of symmetry, behaving at least monoclinically, nullifying the stiffness entries that couple shear in $x_{3}$ direction to those at $x_{1}$ and $x_{2}$ directions. A more detailed discussion about the types of anisotropy and their manifestation in the stiffness tensor is found in Jones [113]. Using this fact $\mathbf{K}$ is simplified to

$$
\begin{aligned}
K_{11}= & \rho c^{2}-A_{1111}-\alpha^{2} A_{1313} \\
K_{22}= & \rho c^{2}-A_{2121}-\alpha^{2} A_{2323} \\
K_{33}= & \rho c^{2}-A_{3131}-\alpha^{2} A_{3333} \\
K_{12}= & K_{21}=-A_{1121}-\alpha^{2} A_{1323} \\
K_{13}= & K_{31}=-\alpha\left(A_{1133}+A_{1331}\right) \\
K_{23}= & K_{32}=-\alpha\left(A_{2133}+A_{2331}\right) .
\end{aligned}
$$

The non trivial solutions are given by the pairs $c$ and $\alpha$ that make the determinant of $\mathbf{K}$ null. Each solution to this polynomial represents a partial wave which combine together in order to form the guided wave packet. For a fixed $c$ the determinant is, a priori, a 6 th order polynomial in $\alpha$. Actually the polynomial is bi-cubic because $\alpha$ is squared in the equations (4.9), (4.11) and (4.12), thus roots present the following properties

$$
\begin{aligned}
& \alpha_{1}=-\alpha_{2}, \\
& \alpha_{3}=-\alpha_{4} \quad \text { and } \\
& \alpha_{5}=-\alpha_{6} \quad .
\end{aligned}
$$

This means that there are six partial wave, one for each root of the $\mathbf{K}$ determinant. This partial waves propagates in the 1-3 plane with different wavenumber ratio, i.e., $\alpha$. The resultant displacement is them the sum of each partial wave field. 


$$
u_{m}=\sum_{q} U_{m_{q}} e^{i \kappa\left(x_{1}+\alpha_{q} x_{3}-c t\right)}
$$

For each partial wave $q$, one can define the displacement ratios

$$
V_{q}=\frac{U_{2}}{U_{1}}
$$

and

$$
W_{q}=\frac{U_{3}}{U_{1}}
$$

Inverting (4.3), expressions for $V_{q}$ and $W_{q}$ are found to be

$$
V_{q}=\frac{K_{11} K_{23}-K_{13} K_{12}}{K_{13} K_{22}-K_{12} K_{23}}
$$

and

$$
W_{q}=\frac{K_{11} K_{23}-K_{13} K_{12}}{K_{12} K_{33}-K_{23} K_{13}}
$$

Using the displacement ratios in (4.15) and (4.16) in the resultant wave equation (4.14), it becomes

$$
\left(\begin{array}{l}
u_{1} \\
u_{2} \\
u_{3}
\end{array}\right)=\sum_{q}\left(\begin{array}{c}
1 \\
V_{q} \\
W_{q}
\end{array}\right) U_{1_{q}} e^{i \kappa\left(x_{1}+\alpha_{q} x_{3}-c t\right)} .
$$

The incremental stress-strain relationship is given by (3.27) or (3.38). By applying these equations in (4.1) one can obtains the incremental stress field. For the boundary conditions of null stresses at the surfaces, one needs to obtain the stress components $T_{33}, T_{13}$ and $T_{23}$. These are given by

$$
T_{i 3}=B_{i 3 k l} \frac{\partial u_{k}}{\partial x_{l}}=B_{i 3 k l} i \kappa\left(\delta_{1 l}+\alpha_{q} \delta_{3 l}\right) u_{k}=i \kappa\left(B_{i 3 k 1}+\alpha_{q} B_{i 3 k 3}\right) u_{k} .
$$

Introducing (4.14) in (4.20) the stress assume the expression:

$$
T_{i 3}=\sum_{q} i \kappa\left(B_{i 3 k 1}+\alpha_{q} B_{i 3 k 3}\right) U_{k_{q}} e^{i \kappa\left(x_{1}+\alpha_{q} x_{3}-c t\right)},
$$

By using the displacement ratios in (4.15) and (4.16) and expanding the implicit sum on $k$ we get

$$
\begin{aligned}
T_{i 3}= & \sum_{q} i \kappa\left[\left(B_{i 311}+\alpha_{q} B_{i 313}\right) U_{1_{q}}+\left(B_{i 321}+\alpha_{q} B_{i 323}\right) U_{2_{q}}\right. \\
& \left.+\left(B_{i 331}+\alpha_{q} B_{i 333}\right) U_{3_{q}}\right] e^{i \kappa\left(x_{1}+\alpha_{q} x_{3}-c t\right)} \\
= & \sum_{q} i \kappa\left[\left(B_{i 311}+\alpha_{q} B_{i 313}\right)+\left(B_{i 321}+\alpha_{q} B_{i 323}\right) V_{q}\right. \\
& \left.+\left(B_{i 331}+\alpha_{q} B_{i 333}\right) W_{q}\right] U_{1_{q}} e^{i \kappa\left(x_{1}+\alpha_{q} x_{3}-c t\right)} .
\end{aligned}
$$

Arranging these three surface stress in a vector leads to

$$
\left(\begin{array}{c}
T_{33} \\
T_{13} \\
T_{23}
\end{array}\right)=\sum_{q} i \kappa\left(\begin{array}{c}
D_{1_{q}} \\
D_{2_{q}} \\
D_{3_{q}}
\end{array}\right) U_{1_{q}} e^{i \kappa\left(x_{1}+\alpha_{q} x_{3}-c t\right)},
$$


where

$$
\begin{aligned}
& D_{1_{q}}=\left(B_{3311}+\alpha_{q} B_{3313}\right)+\left(B_{3321}+\alpha_{q} B_{3323}\right) V_{q}+\left(B_{3331}+\alpha_{q} B_{3333}\right) W_{q} \\
& D_{2_{q}}=\left(B_{1311}+\alpha_{q} B_{1313}\right)+\left(B_{1321}+\alpha_{q} B_{1323}\right) V_{q}+\left(B_{1331}+\alpha_{q} B_{1333}\right) W_{q} \\
& D_{3_{q}}=\left(B_{2311}+\alpha_{q} B_{2313}\right)+\left(B_{2321}+\alpha_{q} B_{2323}\right) V_{q}+\left(B_{2331}+\alpha_{q} B_{2333}\right) W_{q}
\end{aligned}, .
$$

The same argument used for A can be applied here to nullify some entries; (4.24) simplifies to

$$
\begin{aligned}
& D_{1_{q}}=B_{3311}+B_{3321} V_{q}+\alpha_{q} B_{3333} W_{q}, \\
& D_{2_{q}}=\alpha_{q} B_{1313}+\alpha_{q} B_{1323} V_{q}+B_{1331} W_{q}=\alpha_{q}\left(B_{1313}+B_{1323} V_{q}\right)+B_{1331} W_{q} \\
& D_{3_{q}}=\alpha_{q} B_{2313}+\alpha_{q} B_{2323} V_{q}+B_{2331} W_{q}=\alpha_{q}\left(B_{2313}+B_{2323} V_{q}\right)+B_{2331} W_{q}
\end{aligned} .
$$

Applying the free-stress boundary condition at the surface $x_{3}= \pm h=$ $\pm d / 2$, i.e., evaluating (4.23) at $x_{3}= \pm h= \pm d / 2$ and setting it to zero, it results in

$$
\begin{aligned}
\left(\begin{array}{l}
0 \\
0 \\
0
\end{array}\right) & =\sum_{q} i \kappa\left(\begin{array}{c}
D_{1_{q}} \\
D_{2_{q}} \\
D_{3_{q}}
\end{array}\right) U_{1_{q}} e^{i \kappa\left(x_{1} \pm \alpha_{q} h-c t\right)} \\
& =i \kappa e^{i \kappa\left(x_{1}-c t\right)} \sum_{q}\left(\begin{array}{c}
D_{1_{q}} \\
D_{2_{q}} \\
D_{3_{q}}
\end{array}\right) U_{1_{q}} e^{ \pm i \kappa \alpha_{q} h} \\
& =\sum_{q}\left(\begin{array}{c}
D_{1_{q}} \\
D_{2_{q}} \\
D_{3_{q}}
\end{array}\right) U_{1_{q}} e^{ \pm i \kappa \alpha_{q} h} .
\end{aligned}
$$

Note that this equation can be arranged as matrix multiplication

$$
\left[\begin{array}{cccccc}
D_{1_{1}} E_{1} & D_{1_{2}} E_{2} & D_{1_{3}} E_{3} & D_{1_{4}} E_{4} & D_{1_{5}} E_{5} & D_{1_{6}} E_{6} \\
D_{2_{1}} E_{1} & D_{2_{2}} E_{2} & D_{2_{3}} E_{3} & D_{2_{4}} E_{4} & D_{2_{5}} E_{5} & D_{2_{6}} E_{6} \\
D_{3_{1}} E_{1} & D_{3_{2}} E_{2} & D_{3_{3}} E_{3} & D_{3_{4}} E_{4} & D_{3_{5}} E_{5} & D_{3_{6}} E_{6} \\
D_{1_{1}} \tilde{E}_{1} & D_{1_{2}} \tilde{E}_{2} & D_{1_{3}} \tilde{E}_{3} & D_{1_{4}} \tilde{E}_{4} & D_{1_{5}} \tilde{E}_{5} & D_{1_{6}} \tilde{E}_{6} \\
D_{2_{1}} \tilde{E}_{1} & D_{2_{2}} \tilde{E}_{2} & D_{2_{3}} \tilde{E}_{3} & D_{2_{4}} \tilde{E}_{4} & D_{2_{5}} \tilde{E}_{5} & D_{2_{6}} \tilde{E}_{6} \\
D_{3_{1}} \tilde{E}_{1} & D_{3_{2}} \tilde{E}_{2} & D_{3_{3}} \tilde{E}_{3} & D_{3_{4}} \tilde{E}_{4} & D_{35} \tilde{E}_{5} & D_{3_{6}} \tilde{E}_{6}
\end{array}\right]\left(\begin{array}{l}
U_{1_{1}} \\
U_{1_{2}} \\
U_{1_{3}} \\
U_{1_{4}} \\
U_{1_{5}} \\
U_{1_{6}}
\end{array}\right)=\left(\begin{array}{c}
0 \\
0 \\
0 \\
0 \\
0 \\
0
\end{array}\right)
$$

where $E_{q}=e^{+i \kappa \alpha_{q} h}$ and $\tilde{E}_{q}=e^{-i \kappa \alpha_{q} h}$. The non-trivial solutions (where the partial wave displacement amplitudes, $U_{1_{q}}$, are not all null) are found by setting the determinant of the matrix equal to zero.

Evaluating the determinant, and by some algebraic manipulation, the following equations are found. 


$$
\begin{aligned}
& D_{1_{1}}\left(D_{2_{3}} D_{3_{5}}-D_{3_{3}} D_{2_{5}}\right) \cot \left(\frac{\omega d}{2 c} \alpha_{1}\right)+D_{1_{3}}\left(D_{3_{1}} D_{2_{5}}-D_{2_{1}} D_{3_{5}}\right) \cot \left(\frac{\omega d}{2 c} \alpha_{3}\right) \\
& +D_{1_{5}}\left(D_{2_{1}} D_{3_{3}}-D_{3_{1}} D_{2_{3}}\right) \cot \left(\frac{\omega d}{2 c} \alpha_{5}\right)=0 \\
& D_{1_{1}}\left(D_{2_{3}} D_{3_{5}}-D_{3_{3}} D_{2_{5}}\right) \tan \left(\frac{\omega d}{2 c} \alpha_{1}\right)+D_{1_{3}}\left(D_{3_{1}} D_{2_{5}}-D_{2_{1}} D_{3_{5}}\right) \tan \left(\frac{\omega d}{2 c} \alpha_{3}\right) \\
& +D_{1_{5}}\left(D_{2_{1}} D_{3_{3}}-D_{3_{1}} D_{2_{3}}\right) \tan \left(\frac{\omega d}{2 c} \alpha_{5}\right)=0 .
\end{aligned}
$$

The roots of (4.28) and (4.29) are the solutions for the symmetrical and antisymmetrical modes respectively; i.e., the pair $(\omega d, c)$ corresponding to a propagable mode.

\subsection{1}

\section{Note on symmetry assumptions}

In this section it is highlighted some assumptions noticed in Gandhi et al. $[34,96,114]$. Despite of being harmless for their work, it precludes the use of this approach for the initial frame of reference and for non-naturally isotropic medium. It is worth saying that Gandhi et al. work is correct as it is restricted to the natural frame of reference of a naturally isotropic medium, in which all their assumptions are valid.

The first one is found in equation (4.12). Comparing it to the expression presented by Gandhi et al. one can observe that in their equation it has been used $A_{1212}$ instead of $A_{2121}$ at $K_{22}, A_{1313}$ instead of $A_{3131}$ at $K_{33}, A_{1112}$ instead of $A_{1121}$ at $K_{12}$ and $A_{1233}$ instead of $A_{2133}$ at $K_{23}$. As concluded in (3.52a) and $(3.53 \mathrm{a})$ this permutation $\left(A_{i j k l}=A_{j i l k}\right)$ does not hold a priori. However, it does hold in the natural frame for the special case of initially isotropic medium, as stated in (3.54), which is demonstrated in section A.2.1, and further exemplified for uniaxial tensile in section 3.3.1. This means that the above replacement had no effect on the cited work. However, when generalized for the initial reference case, it introduces errors and inconsistencies. In order to correctly obtain the curves at the initial reference one needs to use the precise entries developed in (4.12).

The imprecision is indeed relevant. For instance when using $A_{1212}$ and $A_{1313}$ instead of $A_{2121}$ and $A_{3131}$, the term $s_{j l}^{i} \delta_{i k}$ in (3.34) has its principal components swapped $\left(s_{22}^{i}\right.$ and $s_{33}^{i}$ in the former case and $s_{11}^{i}$ in the correct case), this amount in the stiffness is significant because it is equal to the initial load. The numerical example in (3.55) and (3.56) illustrates it; examples of 
what happens in the dispersion curve if the former expression is used are shown in the next chapter.

The second equation is (4.25). When compared with the work of Gandhi et al. it reveals, again, that some entries on $\mathbf{D}$ are different here from what is there. These are, $B_{3312}$ instead of $B_{3321}$ in $D_{3_{1}}, B_{1323}$ instead of $B_{2313}$ and $B_{1332}$ instead of $B_{2331}$ in $D_{3 q}$. This makes no effect on the initial frame as $\mathbf{B}^{i}$ presents full symmetry. For the natural frame, used by Gandhi et al., this are not permutable a priori. However, at least for the case of naturally isotropic material, one can easily compute these specific entries and conclude that they are equal. Again, this leads to no effect in their work, but it may not allow a generalization for naturally anisotropic medium. If one wants to safely work with anisotropic media in their natural configuration, the expression (4.24) should be used.

\section{2}

\section{Computational routine}

There is no analytical solution for solving (4.28) and (4.29) as a function of $c$ and $\omega d$. Thus a computational routine, as zero-finding [115], should be used. In this section the main procedure is summarized. More details in its implementation are found in $[34,96]$. The procedure can be summarized as follows.

1. Fix a velocity values, say $c_{0}$.

2. Calculate the determinant of $\mathbf{K}$ in (4.11) or (4.12) and find the six values of $\alpha_{q}$ that render it null.

3. Calculate $V_{q}$ and $W_{q}$ for all values of $\alpha_{q}$ by means of (4.17) and (4.18), respectively.

4. Calculate the vector $\mathbf{D}_{q}$ in (4.24) or (4.25) for all the six values of $q$ using the $\alpha_{q}, V_{q}$ and $W_{q}$.

5. Assemble the functions (4.28) and (4.29) using the the entries of $\mathbf{D}_{q}$.

6. Seek for values of $\omega d$ which nullify (4.28) and (4.29). This should be done by a zero finding routine. The possible roots, say $\omega d_{i}$, together with $c_{0}$ form a pair of frequency $\times$ thickness-velocity for mode $i$.

7. Repeat steps 2 to 6 for other fixed velocity.

The procedure schematized above allows one to find all the frequency $\times$ thickness-velocity for the possible modes. 


\section{3}

\section{Particular case of longitudinal applied load in naturally isotropic medium}

In the case where the initial stress is uniaxial in the direction of the wave propagation in an naturally isotropic material, some simplifications can be introduced in the development of section 4.1. This is done in this section because this particular case is of practical interest in the sequel.

This simplifications were checked to match the very recent work of Mohabuth et al. [102] in which Lamb wave propagates in uniaxial stressed plate under the initial frame of reference. In that work the acoustoelastic background is different. It is developed over the large acoustoelastic theory proposed by Ogden $[85,88,116]$. Basically the stiffness tensor is found by other means.

Under longitudinal stress (4.12) can be further simplified because some entries of $\mathbf{A}$ are null, resulting in

$$
\begin{aligned}
K_{11} & =\rho c^{2}-A_{1111}-\alpha^{2} A_{1313}, \\
K_{22} & =\rho c^{2}-A_{2121}-\alpha^{2} A_{2323}, \\
K_{33} & =\rho c^{2}-A_{3131}-\alpha^{2} A_{3333}, \\
K_{12}= & K_{21}=0 \\
K_{13}= & K_{31}=-\alpha\left(A_{1133}+A_{1331}\right), \\
K_{23}= & K_{32}=0 .
\end{aligned}
$$

Consequently, the matrix $K$ naturally decouples as it can be seen below where (4.3) is rewritten as

$$
\left[\begin{array}{ccc}
K_{11} & 0 & K_{13} \\
0 & K_{22} & 0 \\
K_{13} & 0 & K_{13}
\end{array}\right]\left(\begin{array}{l}
U_{1} \\
U_{2} \\
U_{3}
\end{array}\right)=\left(\begin{array}{l}
0 \\
0 \\
0
\end{array}\right)
$$

Decoupling it yields

$$
\begin{aligned}
{\left[\begin{array}{ll}
K_{11} & K_{13} \\
K_{13} & K_{13}
\end{array}\right]\left(\begin{array}{l}
U_{1} \\
U_{3}
\end{array}\right) } & =\left(\begin{array}{l}
0 \\
0
\end{array}\right) \quad \text { and } \\
K_{22} U_{2} & =0
\end{aligned}
$$

Equations (4.32b) implies in the Shear Horizontal (SH) waves, which for this case decouple from the Lamb wave, as in isotropic medium. One may note that defining $V$, as in (4.15), makes no sense for this case. However, $W$ is still defined in same way as in (4.16), but it assumes the expression

$$
W_{q}=-\frac{K_{33}}{K_{13}} .
$$

Developing the determinant of (4.32a) leads to 


$$
\operatorname{det}(\mathbf{K})=K_{11} K_{33}-K_{13}^{2}
$$

which is a forth order polynomial in $\alpha$; not sixth order any more. Actually, it is a bi-quadratic polynomial, i.e.,

$$
\operatorname{det}(\mathbf{K})=K_{11} K_{33}-K_{13}=P_{4} \alpha^{4}+P_{2} \alpha^{2}+P_{0}
$$

Introducing the entries of $\mathbf{K}$ from (4.30) into (4.34) allows one to calculate the polynomial coefficients. The values are

$$
\begin{aligned}
P_{4} & =A_{1313} A_{3333} \\
P_{2} & =-\rho c^{2}\left(A_{1313}+A_{3333}\right)+A_{1313} A_{3131}+A_{1111} A_{3333} \\
& -A_{1133} A_{3113}-A_{1133} A_{3113}-A_{1133} A_{3311} \\
& -A_{1331} A_{3113}-A_{1331} A_{3311}, \\
P_{0} & =\rho^{2} c^{4}-\rho c^{2}\left(A_{1111}+A_{3131}\right)+A_{1111} A_{3131}
\end{aligned}
$$

Because the polynomial is bi-quadratic, the roots are still symmetrical, i.e., the first two lines of (4.13) continue to hold.

One should retain only the first and second entries on the vector $\mathbf{D}$, which become

$$
\begin{aligned}
& D_{1_{q}}=B_{3311}+\alpha_{q} B_{3333} W_{q} \\
& D_{2_{q}}=\alpha_{q} B_{1313}+B_{1331} W_{q} .
\end{aligned} .
$$

and the matrix in (4.27) reduces to

$$
\left[\begin{array}{cccc}
D_{1_{1}} E_{1} & D_{1_{2}} E_{2} & D_{1_{3}} E_{3} & D_{1_{4}} E_{4} \\
D_{2_{1}} E_{1} & D_{2_{2}} E_{2} & D_{2_{3}} E_{3} & D_{2_{4}} E_{4} \\
D_{1_{1}} \tilde{E}_{1} & D_{1_{2}} \tilde{E}_{2} & D_{1_{3}} \tilde{E}_{3} & D_{1_{4}} \tilde{E}_{4} \\
D_{2_{1}} \tilde{E}_{1} & D_{2_{2}} \tilde{E}_{2} & D_{2_{3}} \tilde{E}_{3} & D_{2_{4}} \tilde{E}_{4}
\end{array}\right]\left(\begin{array}{l}
U_{1_{1}} \\
U_{1_{2}} \\
U_{1_{2}} \\
U_{1_{3}}
\end{array}\right)=\left(\begin{array}{l}
0 \\
0 \\
0 \\
0
\end{array}\right) .
$$

Finally the equations for symmetrical and antisymmetrical roots in (4.28) and (4.29) reduce to

$$
\begin{aligned}
& D_{1_{1}} D_{2_{3}} \cot \left(\frac{\omega d}{2 c} \alpha_{1}\right)-D_{1_{3}} D_{2_{1}} \cot \left(\frac{\omega d}{2 c} \alpha_{3}\right)=0 \\
& D_{1_{1}} D_{2_{3}} \tan \left(\frac{\omega d}{2 c} \alpha_{1}\right)-D_{1_{3}} D_{2_{1}} \tan \left(\frac{\omega d}{2 c} \alpha_{3}\right)=0 .
\end{aligned}
$$

The procedure is the same as the one exposed in section 4.2 , by using the equations derived here instead of the ones for the general case. 


\section{4}

\section{Partial wave technique for anisotropic medium}

The above procedure can be used for a general anisotropic medium, without any pre-stress. This is done simply using the normal second order stiffness tensor $\mathbf{C}$ instead of both tensors, $\mathbf{A}$ and $\mathbf{B}$, on the above procedure. As the normal stiffness tensor possesses the full symmetries, several simplifications are possible; it can be expressed by means of the conventional Voigt notation.

The conventional Voigt notations allows one to write a fourth order tensor, $C_{i j k l}$, in a $6 \times 6$ matrix, $C_{m n}$, by means of the tensor symmetries. In this notation $m$ represent the two indexes $i j$ together and $n$ represents $k l$, by the following rule.

Table 4.1: Voigt notation.

\begin{tabular}{c|c}
$i j$ or $k l$ & $m$ or $n$ \\
\hline 11 & 1 \\
22 & 2 \\
33 & 3 \\
23 or 32 & 4 \\
13 or 31 & 5 \\
12 or 21 & 6
\end{tabular}

Applying this simplifications to the partial wave technique developed at section 4.1, i.e., setting $\mathbf{A}=\mathbf{B}=\mathbf{C}$, considering the symmetries and using Voigt notation, the $\mathbf{K}$ matrix and $\mathbf{D}$ vector become equal to

$$
\begin{aligned}
K_{11} & =\rho c^{2}-C_{11}-2 \alpha C_{15}-\alpha^{2} C_{55}, \\
K_{22} & =\rho c^{2}-C_{66}-2 \alpha C_{64}-\alpha^{2} C_{44}, \\
& =\rho c^{2}-C_{66}-2 \alpha C_{46}-\alpha^{2} C_{44}, \\
K_{33} & =\rho c^{2}-C_{55}-2 \alpha C_{53}-\alpha^{2} C_{33}, \\
& =\rho c^{2}-C_{55}-2 \alpha C_{35}-\alpha^{2} C_{33}, \\
K_{12}=K_{21} & =-C_{16}-\alpha\left(C_{14}+C_{56}\right)-\alpha^{2} C_{54} \\
& =-C_{16}-\alpha\left(C_{14}+C_{56}\right)-\alpha^{2} C_{45}, \\
K_{13}=K_{31} & =-C_{15}-\alpha\left(C_{13}+C_{55}\right)-\alpha^{2} C_{53}, \\
& =-C_{15}-\alpha\left(C_{13}+C_{55}\right)-\alpha^{2} C_{35}, \\
K_{23}=K_{32} & =-C_{65}-\alpha\left(C_{63}+C_{45}\right)-\alpha^{2} C_{43}, \\
& =-C_{56}-\alpha\left(C_{36}+C_{45}\right)-\alpha^{2} C_{34},
\end{aligned},
$$

and 


$$
\begin{aligned}
D_{1_{q}} & =\left(C_{31}+\alpha C_{35}\right)+\left(C_{36}+\alpha C_{34}\right) V_{q}+\left(C_{35}+\alpha C_{33}\right) W_{q} \\
& =\left(C_{13}+\alpha C_{35}\right)+\left(C_{36}+\alpha C_{34}\right) V_{q}+\left(C_{35}+\alpha C_{33}\right) W_{q}, \\
D_{2_{q}} & =\left(C_{51}+\alpha C_{55}\right)+\left(C_{56}+\alpha C_{54}\right) V_{q}+\left(C_{55}+\alpha C_{53}\right) W_{q} \\
& =\left(C_{15}+\alpha C_{35}\right)+\left(C_{36}+\alpha C_{34}\right) V_{q}+\left(C_{35}+\alpha C_{33}\right) W_{q}, \\
D_{3_{q}} & =\left(C_{41}+\alpha C_{45}\right)+\left(C_{46}+\alpha C_{45}\right) V_{q}+\left(C_{45}+\alpha C_{43}\right) W_{q} \\
& =\left(C_{14}+\alpha C_{45}\right)+\left(C_{46}+\alpha C_{45}\right) V_{q}+\left(C_{45}+\alpha C_{43}\right) W_{q} .
\end{aligned}
$$

These two last equations are exactly the ones presented in the work of Nayfeh and Chimenti [117] and recently reviewed by Verma [118], where the partial wave technique was applied to obtain the dispersion curves for a general anisotropic medium.

All the remainder procedure presented in in section 4.1 is the same. In other words the case of anisotropic waves in plates can be viewed as particular case of the procedure for acoustoelastic waves in plates where both tensor coincide and possess the full symmetries.

\subsection{1}

\section{Approximation for acoustoelastic case using Effective Elastic Constants}

Applying the concept of Effective Elastic Constants (EEC) one can use the equations (4.41) and (4.42) in order to obtain an approximated procedure for the dispersion relationship. The EEC can be basically summarized by choosing one of the possible entries on $A_{i j k l}$ in order to assemble $C_{m n}$, the stiffness tensor written using Voigt notation [29]. As stated above A do not possesses the necessary full symmetries, implying that the conventional Voigt tensor can never be built (only the extended Voigt notation presented in Table 3.1). The entries where the symmetries fail are said to split. The procedure to obtain an approximate Voigt tensor is to choose between one of the split entries.

The choice must be done consistently. That is, if the loading process brings some structural symmetry (as the case of monoclinic symmetry, due to biaxial loading, treated in section 4.1 or even transverse isotropic symmetry, due to uniaxial longitudinal loading, treated in section 4.3) the EEC tensor has to maintain these symmetries. For example, in the case of longitudinal loading in $x_{1}$ direction, if one chooses $C_{55}=A_{1313}$, then $C_{66}=A_{1212}$, and not $A_{1221}$ or even $A_{2121}$ as this can introduce an artificial stiffness asymmetry, degrading the approximation.

For a naturally isotropic material under longitudinal tensile stress, there are two possible EECs choices. The EEC1 case is defined as the Voigt notation build by choosing the index $m$ and $n$ as 32, 31 or 21, whereas the EEC2 
case is defined as choosing $m$ as 32,31 or 21 and $n$ as 23, 13 or 12 (or viceversa). Continuing the numerical example of an aluminum body under stress of $\sigma_{11}=120 M P a$, presented in section 3.3.1, the two EECs cases assume the following values for the natural frame

$$
\mathbf{A}_{\text {EEC1 }}^{\mathbf{n}}={ }^{2}=33\left[\begin{array}{cccccc}
11 & 22 & 33 & 32 & 31 & 21 \\
32 & 31 \\
105.91 & 54.51 & 54.51 & 0 & 0 & 0 \\
54.51 & 108.24 & 55.06 & 0 & 0 & 0 \\
54.51 & 55.06 & 108.24 & 0 & 0 & 0 \\
0 & 0 & 0 & 26.59 & 0 & 0 \\
0 & 0 & 0 & 0 & 26.31 & 0 \\
0 & 0 & 0 & 0 & 0 & 26.31
\end{array}\right] G P a
$$

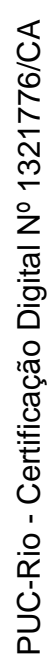

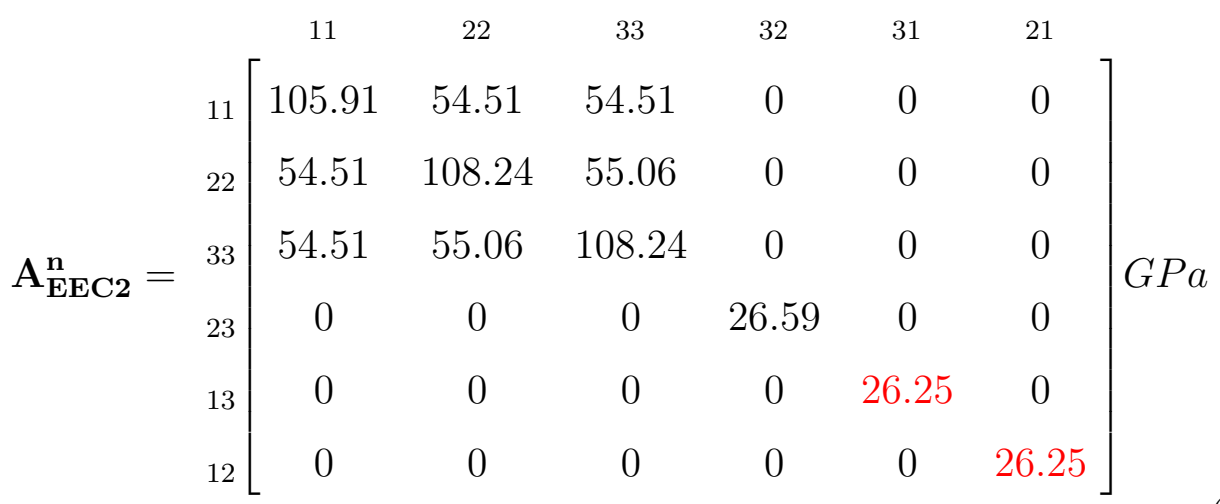

and for the initial frame

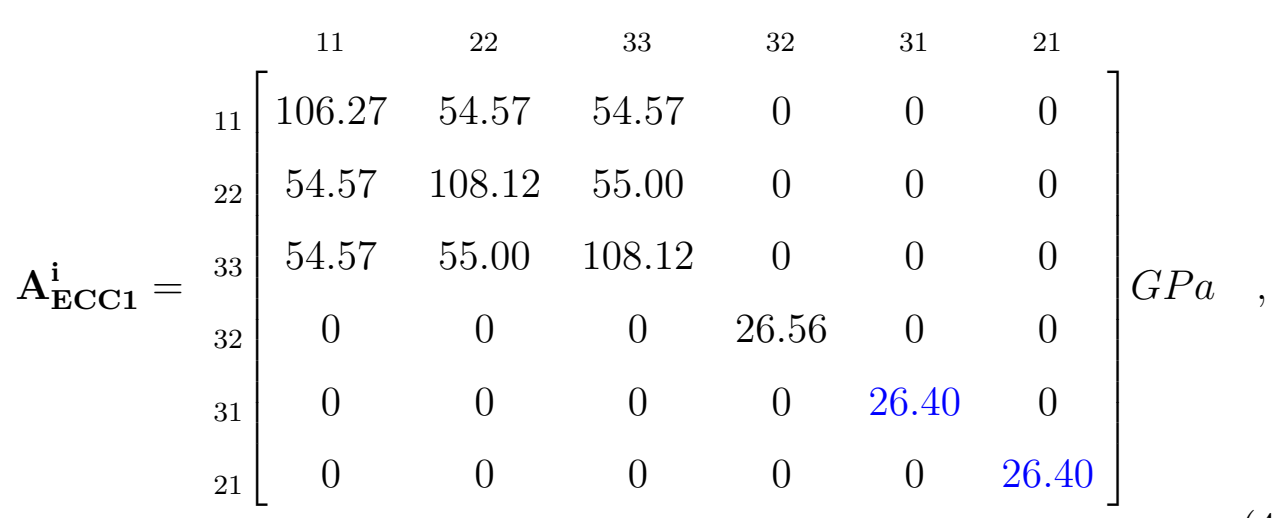




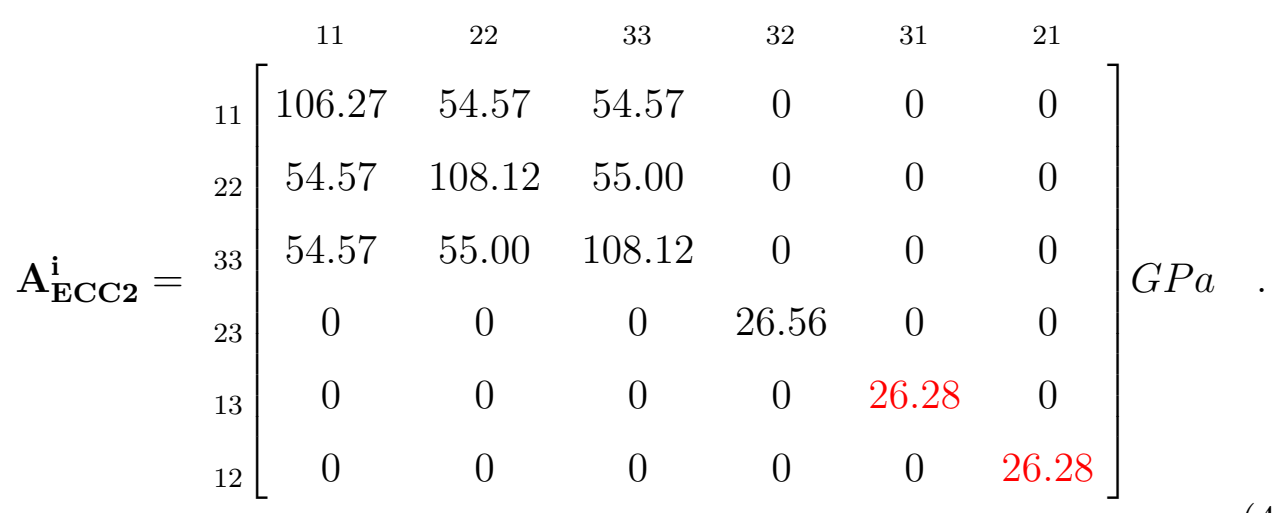

Note that the use of EEC in order to trace a dispersion curve for an equivalent anisotropic medium brings inherent errors due to the loss of information. The asymmetry in the stiffness tensor is a consequence of the load. The correct procedure exposed in section 4.1 makes distinctions between the stiffness entries, as for example in (4.12) where there is $A_{1313}$ and $A_{1331}$. The choice between one of the split entries will never depict the phenomenon as precise as the original approach. Other source of imprecision is the merge of $\mathbf{A}$ and $\mathbf{B}$. In the next chapter dispersion curves obtained by means of EEC approximation are confronted with those by the precise framework in order to illustrate the approximation validity and its errors. The error of the EEC dispersion curves, related to the exact theoretical solution, is used in order to choose between EEC cases.

The use of the EEC in order to calculate the dispersion curves may appears with no purpose as the precise procedure has been presented at the beginning of the chapter. The reason for considering it here is to provide the necessary theoretical background in order to compare the acoustoelastic Finite Element results of chapter 6 . 


\section{5}

\section{Acoustoelastic Lamb waves dispersion curves}

In this chapter it is shown the dispersion curves obtained with the procedure exposed at chapter 4 for both frames of reference. As the main interest here is longitudinal load, only the case of tensile stress in the direction of wave propagation is analyzed. The approximation for the effective elastic case is compared with the exact solution.

The calculations are performed for an aluminum plate with the constants presented in table 3.2. The frequency times thickness interval used for calculation was from 0 up to $10 \mathrm{MHz} \times \mathrm{mm}$ in steps of $1 \mathrm{kHz} \times \mathrm{mm}$ and the velocity interval from 0 up to $10000 \mathrm{~m} / \mathrm{s}$ in steps of $1 \mathrm{~m} / \mathrm{s}$. In order to illustrate dispersion curves behavior under longitudinal stress, several calculations were performed for different stress values. The stress and corresponding strain state used at each calculation are shown in table 5.1. It is presented the longitudinal stress $\left(\sigma_{11}\right)$ and the normal strains $\left(\varepsilon_{11}, \varepsilon_{22}, \varepsilon_{33}\right)$, as all the remaining stresses and strains are null.

Table 5.1: Stress and strain states used at calculations.

\begin{tabular}{c|ccc}
\hline $\begin{array}{c}\sigma_{11} \\
\mathrm{MPa}\end{array}$ & $\begin{array}{c}\varepsilon_{11} \\
\mu \mathrm{m} / \mathrm{m}\end{array}$ & $\begin{array}{c}\varepsilon_{22} \\
\mu \mathrm{m} / \mathrm{m}\end{array}$ & $\begin{array}{c}\varepsilon_{33} \\
\mu \mathrm{m} / \mathrm{m}\end{array}$ \\
\hline 0 & 0 & 0 & 0 \\
30 & 423 & -143 & -143 \\
60 & 847 & -285 & -285 \\
90 & 1270 & -428 & -428 \\
120 & 1693 & -571 & -571 \\
150 & 2117 & -714 & -714 \\
\hline
\end{tabular}

\section{1}

\section{Natural reference frame}

The acoustoelastic dispersion curves, computed at the natural frame of reference, are investigated here. The $A 0$ and $S 0$ modes are investigated in details; at the end of the section higher order modes are studied as well. 


\subsection{1 \\ A0 mode}

The phase velocity curves for the $A 0$ mode is shown in Fig. 5.1.a for the full frequency range, from $D C$ up to $10 \mathrm{MHz}$. As it can be seen, the dispersion change under stress is relatively small compared to the velocity value at any stress condition. The most significant change occurs at low frequencies as detailed in Fig. 5.1.b, where the higher the tensile stress the higher the velocity. Under tensile stress the velocities tend to a limit non-zero value as the frequency approaches to zero. This phenomenum is also present when addressing the problem by the Euler-Bernoulli beam theory [119] imposing an axial traction in the motion equation. This theory is a good approximation for very low frequency as noticed in [33].

This behavior is better noticeable expressing the curves in terms of the velocity difference given by

$$
\Delta c_{p h, g r}(f, \sigma)=c_{p h, g r}(f, \sigma)-c_{p h, g r}(f, 0)
$$

or by the relative difference as

$$
\frac{\Delta c_{p h, g r}(f, \sigma)}{c_{p h, g r}(f, 0)}
$$

where $c_{p h, g r}(f, \sigma)$ is either the phase (subscript ${ }_{p h}$ ) or group velocity (subscript $g r)$ at the current stress state, $\sigma$, and $c(f, 0)_{p h, g r}$ is the velocity at null stress state. Fig. 5.1.d and 5.1.g show the phase velocity differences for low frequencies. It is clear that the difference tends to the velocity $D C$ value, because for null stress the velocity is null at $D C$ frequency. Thus, the relative difference tends to infinity at $D C$.

On the contrary, for high frequencies (Fig. 5.1.c) the behavior is the opposite, i.e., the higher the tensile stress, the lower the velocity, suggesting that a zero-crossing must occur somewhere in the frequency range. This is detailed in Fig. 5.1.e where the zero cross frequency-thickness is about $246 \mathrm{kHz} \times \mathrm{mm}$. The remainder of the frequency-thickness spectrum, excluding the very low frequency range, is exhibited in Fig. 5.1.f. The huge velocity difference that happens in frequencies below the cross frequency (Fig. 5.1.g) is replaced by differences at the order of some tenths of a percent in magnitude, as shown in Fig. 5.1.h, but negative in sign, i.e., the velocity decrease with tensile stress.

The group velocity behavior has also been calculated, by means of expression 2.18, and is shown in Fig. 5.2.a. The $D C$ value of group and phase velocity must be the same (as it can be concluded from (2.18)). This is reported in Fig. 5.2.b, where the velocity increases as the tensile load increases, for a very 


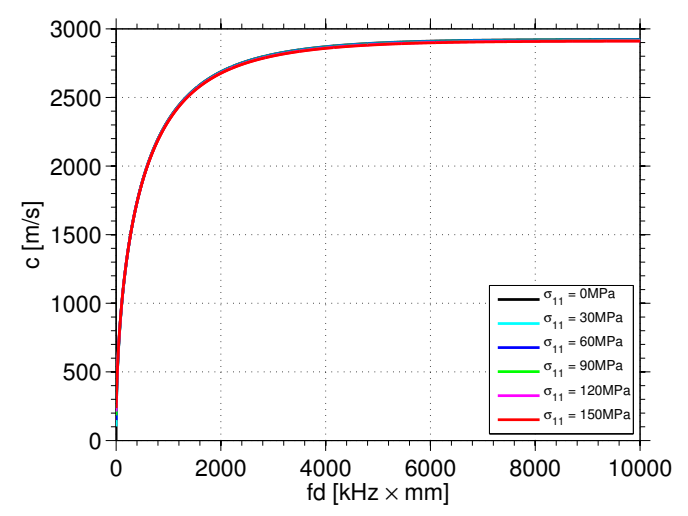

(a)

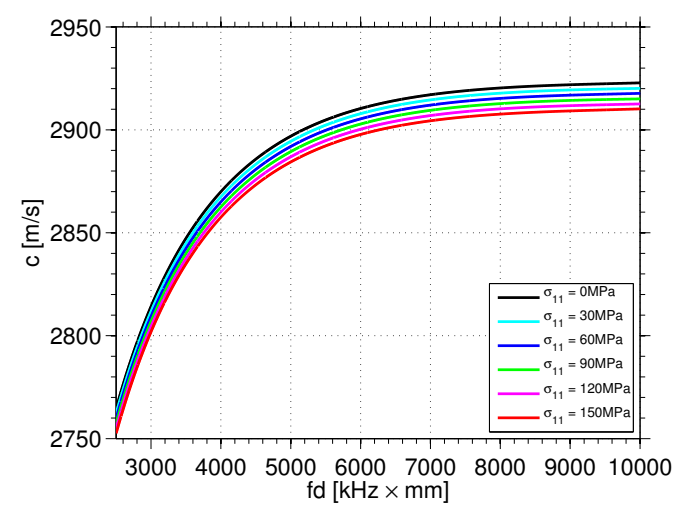

(c)

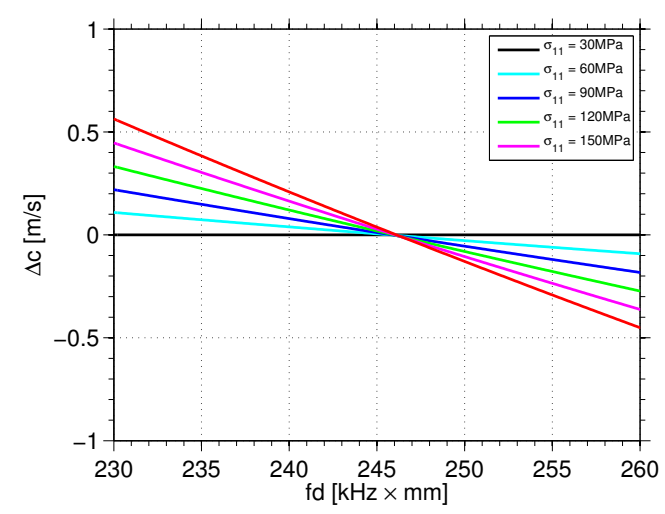

(e)

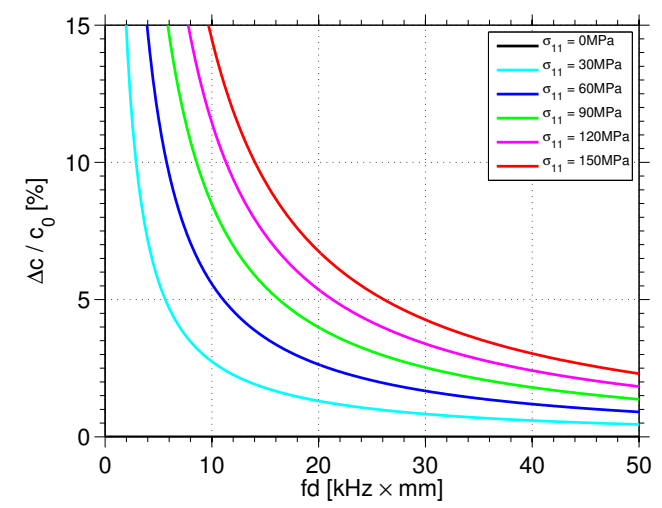

(g)

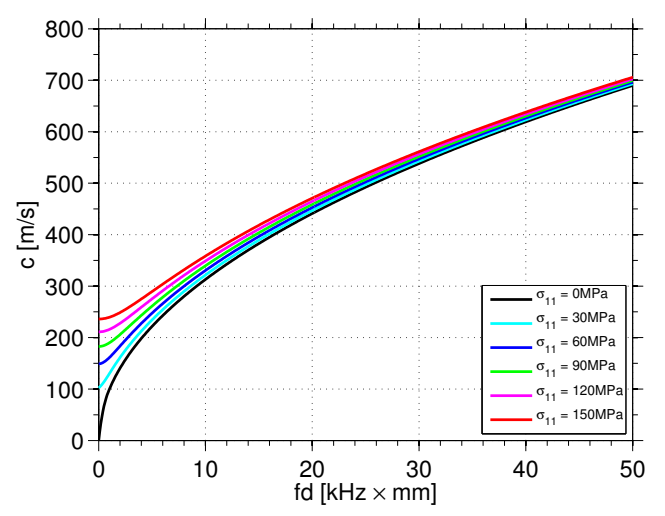

(b)

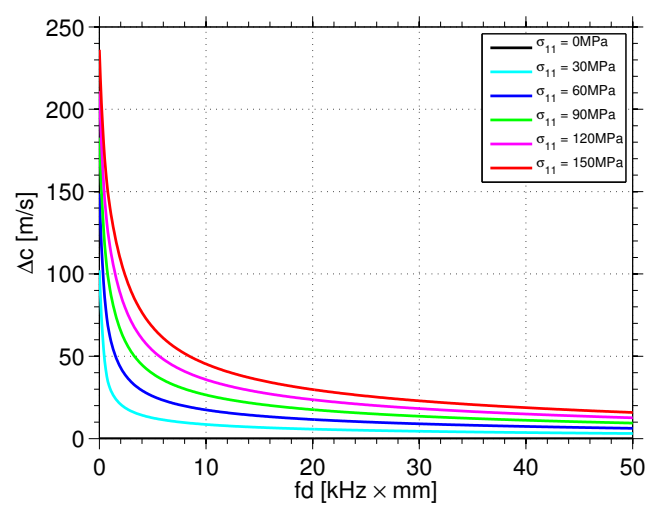

(d)

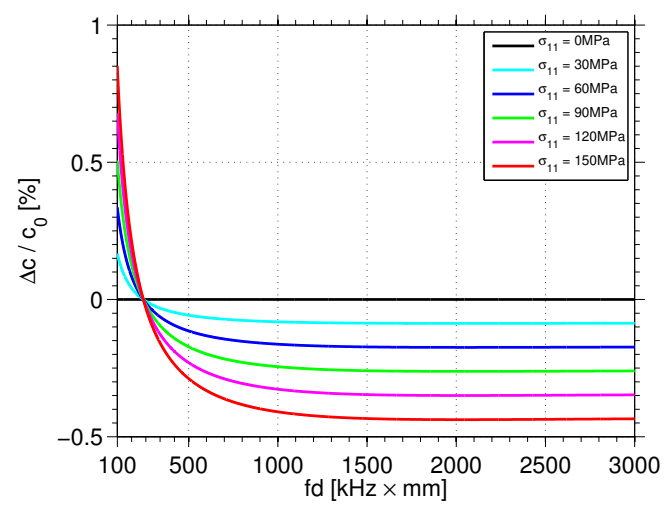

(f)

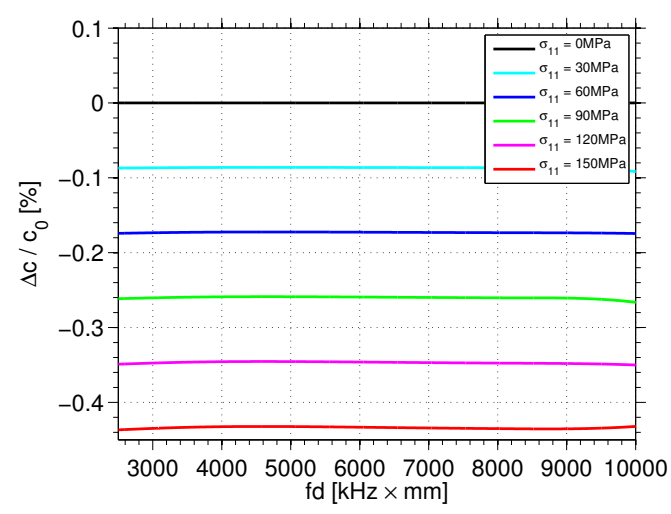

(h)

Figure 5.1: Acoustoelastic phase velocity dispersion curves for $A 0$ at natural frame of reference under tensile stress. (a) Full frequency range. (b) Low frequencies. (c) High frequencies. (d) Velocity difference from null stress condition, low frequencies. (e) Velocity difference, zero crossing region. (f) Velocity difference, mid and high frequencies. (g) Velocity relative difference, low frequencies. (h) Velocity relative difference, high frequencies. 
short and extremely low frequency range. It is then followed by a quick zero crossing around $1.2 \mathrm{kHz} \times \mathrm{mm}$ for the group velocity difference (Fig. 5.2.c and 5.2.e), reaching a maximum negative difference. Then the difference increases, but still negative for the remainder of the frequency spectrum (Fig. 5.2.d). For high frequencies the group velocity difference (Fig. 5.2.f) reaches about the same values as the phase velocity difference, because this is a low dispersive region implying that phase and group velocities tend to be equal.

It is convenient to express the velocities behavior in terms of the its sensitivity to tensile stress or strain, as done by Chen and Wilcox [33]. It is defined by the relative velocity difference per imposed tensile stress or strain. Thus

$$
s_{g r, p h}^{\sigma}(f)=\frac{\Delta c_{g r, p h}\left(f, \sigma_{11}\right) / c_{g r, p h}(f, 0)}{\sigma_{11}},
$$

for the tensile stress sensitivity and

$$
s_{g r, p h}^{\varepsilon}(f)=\frac{\Delta c_{g r, p h}\left(f, \varepsilon_{11}\right) / c_{g r, p h}(f, 0)}{\varepsilon_{11}},
$$

for the tensile strain sensitivity, where the velocity is treated as a function of strain instead of stress. This definition represents the fractional change in velocity to applied stress, or strain. For the special case treated here of uniaxial tensile stress the sensitivities are related by,

$$
s_{g r, p h}^{\varepsilon}(f)=E s_{g r, p h}^{\sigma}(f)
$$

where $E$ is the Young modulus. One can notice that if the sensitivity is constant for a range of stresses (resp. strain), i.e., does not depend on stress (resp. strain), then the relationship between velocity and stress (resp. strain) is linear, otherwise not linear.

It is also used the length normalized time-of-flight sensitivity [107] instead of group velocity sensitivity. It is defined as

$$
s_{t / \ell}^{\sigma}(f)=\frac{\Delta t\left(f, \sigma_{11}\right) / \ell}{\sigma_{11}}
$$

or

$$
s_{t / \ell}^{\varepsilon}(f)=\frac{\Delta t\left(f, \varepsilon_{11}\right) / \ell}{\varepsilon_{11}},
$$

where $\ell$ is the natural distance, $t$ the time-of-flight for this distance and $\Delta t$ the time-of-flight shift. This sensitivity can be related to the group velocity sensitivity. In order to achieve this relationship, it is convenient to develop the variation of group velocity. In the natural frame of reference the elongation is not considered, and so

$$
\Delta c_{g r}=-\ell \frac{\Delta t}{t^{2}}=-\ell \frac{\Delta t}{t^{2}} \frac{\ell^{2}}{\ell^{2}}=-\ell \frac{\Delta t}{\ell^{2}} \frac{\ell^{2}}{t^{2}}=-\frac{\Delta t}{\ell} c_{g r}^{2} .
$$

Applying this last equality into (5.3), we get 


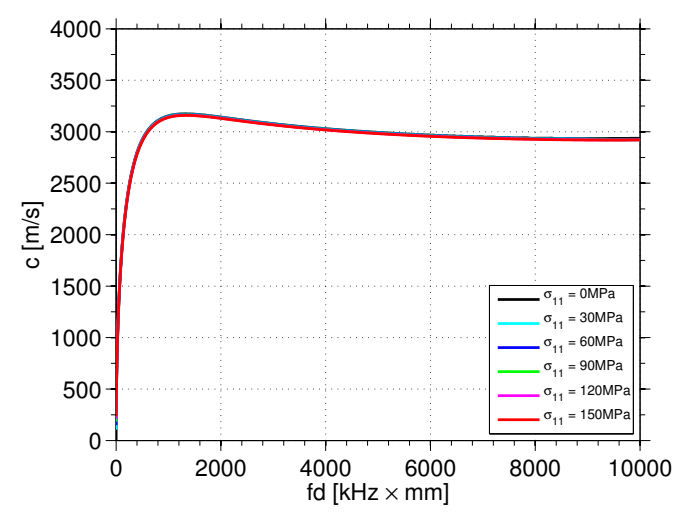

(a)

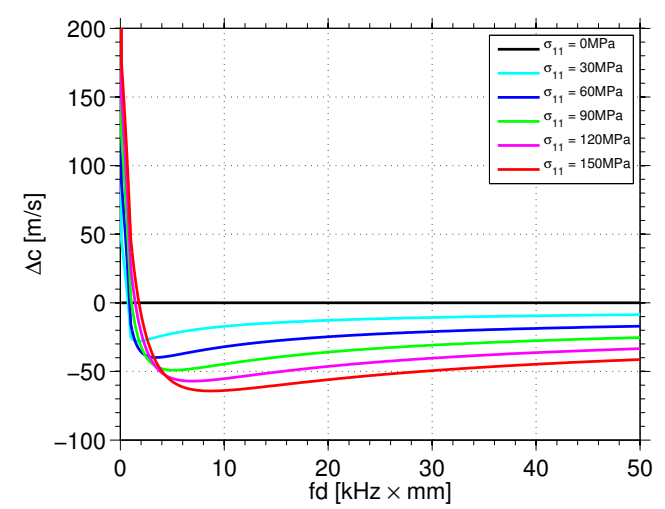

(c)

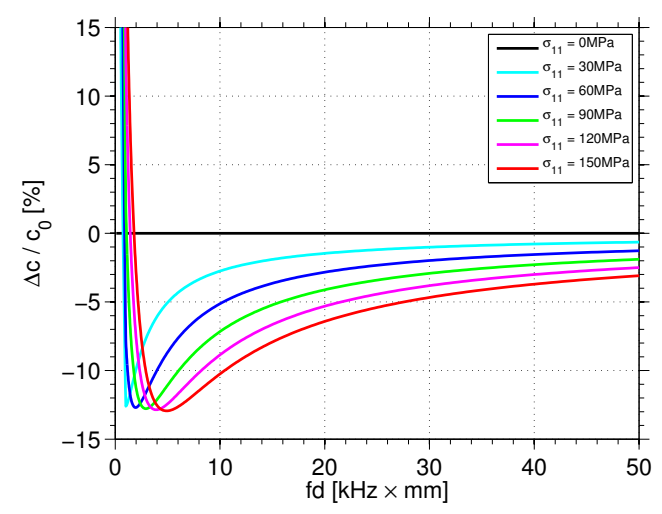

(e)

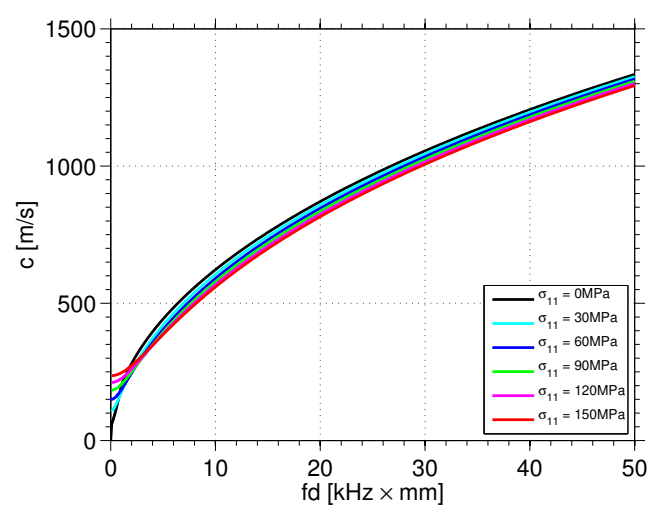

(b)

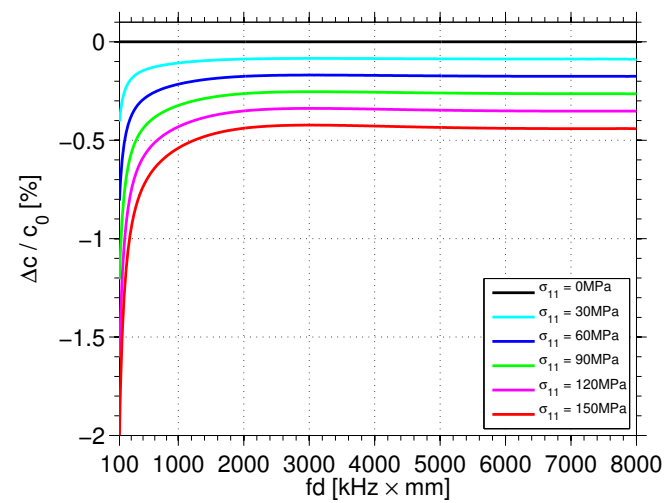

(d)

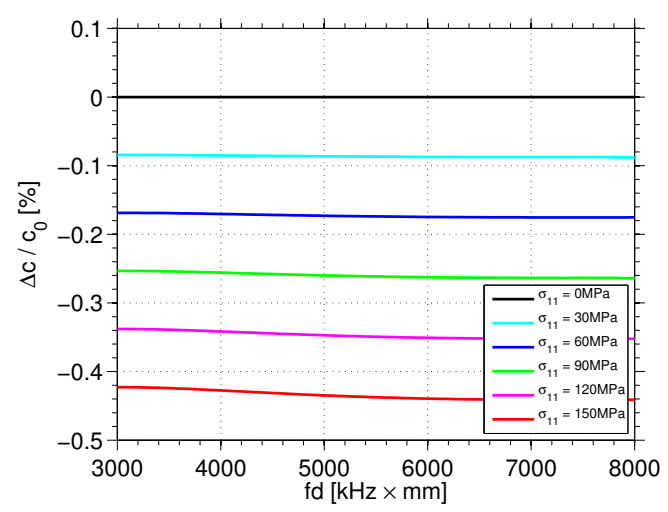

(f)

Figure 5.2: Acoustoelastic group velocity dispersion curves for $A 0$ at natural frame of reference under tensile stress. (a) Full frequency range. (b) Low frequencies. (c) Velocity difference from null stress condition, low frequencies. (d) Velocity relative difference, mid and high frequencies. (e) Velocity relative difference, low frequencies. (f) Velocity relative difference, high frequencies.

$$
\begin{aligned}
s_{g r, p h}^{\sigma}(f) & =\frac{\left(-\Delta t\left(f, \sigma_{11}\right) / \ell\right) c_{g r, p h}(f, 0)}{\sigma_{11}} \\
& =-s_{t / \ell}^{\sigma}(f) \times c_{g r, p h}(f, 0),
\end{aligned}
$$

and equivalently for (5.4) 


$$
s_{g r, p h}^{\varepsilon}(f)=-s_{t / \ell}^{\varepsilon}(f) \times c_{g r, p h}(f, 0) .
$$

The sensitivity curves for the $A 0$ mode are shown in Fig. 5.3.a. and 5.3.c. for phase and group velocity, respectively. It is used a log scale for the frequency in order to ease visualization at very low frequencies, below $1 \mathrm{kHz} \times \mathrm{mm}$. It is visible that the sensitivity for each loading condition differ at low frequencies, meaning that the velocity-stress relationship is nonlinear for frequencies below $1 \mathrm{kHz} \times \mathrm{mm}$. At this range the sensitivity is huge, reaching up to 12000 per strain (or $0.012 /(\mu \mathrm{m} / \mathrm{m})$ ) or $0.17 / M P a$. For higher frequencies the curves do overlap, implying a linear relationship, this is even more clear in Fig. 5.3.b. and 5.3.d. where attention is given to low sensitivity at middle to high frequency range, in a linear frequency scale.

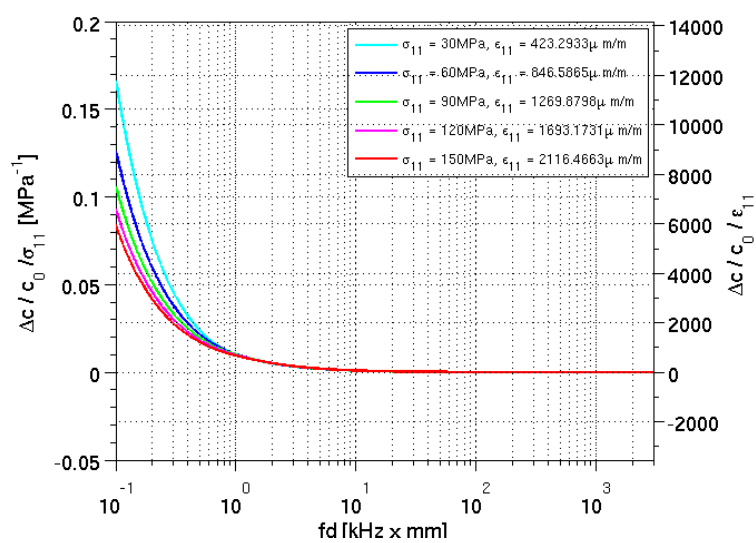

(a)

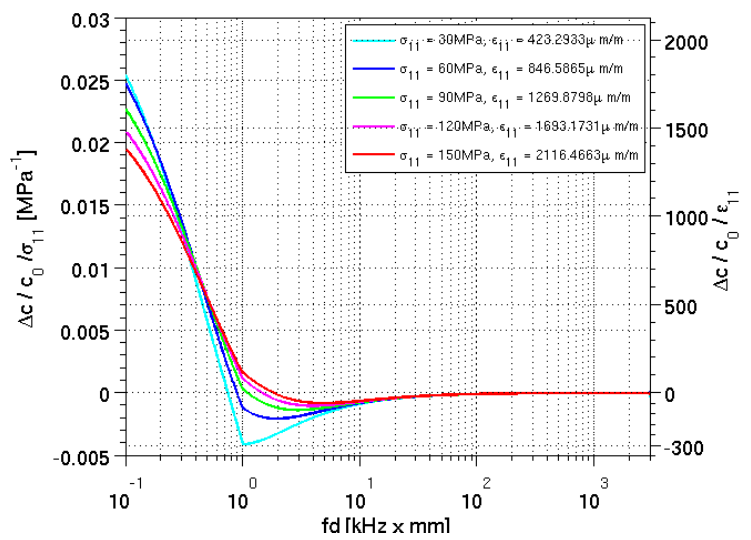

(c)

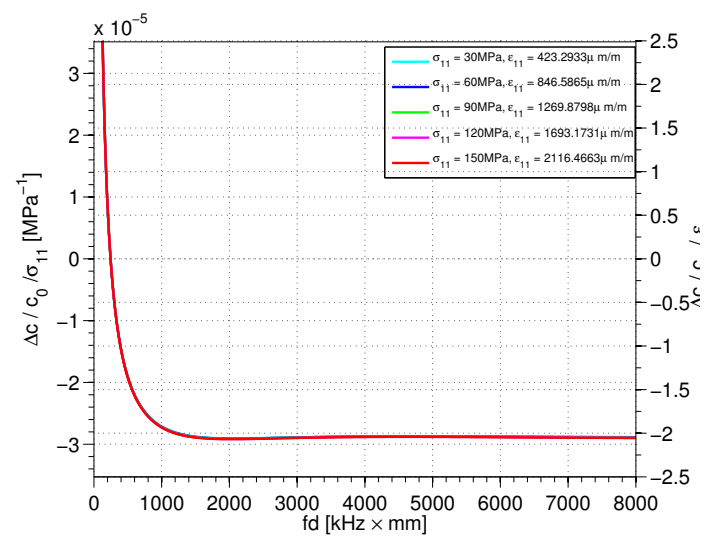

(b)

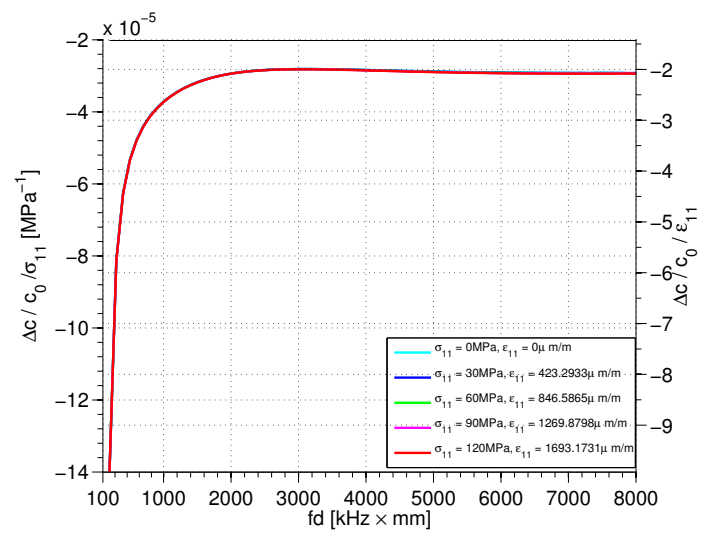

(d)

Figure 5.3: Acoustoelastic velocity sensitivity for $A 0$ mode at natural frame of reference under tensile stress. (a) Phase velocity sensitivity in logarithmic frequency scale. (b) Phase velocity sensitivity in linear frequency scale. (c) Group velocity sensitivity in logarithmic frequency scale. (d) Group velocity sensitivity in linear frequency scale. 


\section{1 .2}

\section{S0 mode}

The $S 0$ mode velocity behavior under stress is simpler. Fig. 5.4.a shows the velocity curves for each tensile load. The velocity difference and relative velocity difference are presented in Fig. 5.4.b and 5.4.c. This mode does not present the huge difference at low frequency, as $A 0$, there is no zero crossing, and the difference is always negative; i.e., for tensile loading, phase velocity decreases as load increases. For this mode, the low frequency regions is almost non-dispersive and the velocity relative difference is almost constant up to $2000 \mathrm{kHz} \times \mathrm{mm}$ (see Fig. 5.4.c). Comparing the modes behavior at the frequency range above the $A 0$ zero crossing, $S 0$ mode present higher difference than $A 0$ mode (see Fig. 5.1.f and 5.4.c). At the high frequency region, above $3000 \mathrm{kHz} \times \mathrm{mm}$ both differences, $A 0$ and $S 0$, tend to the same values (and differences) as both modes velocity converge to the Rayleigh surface wave velocity [14].

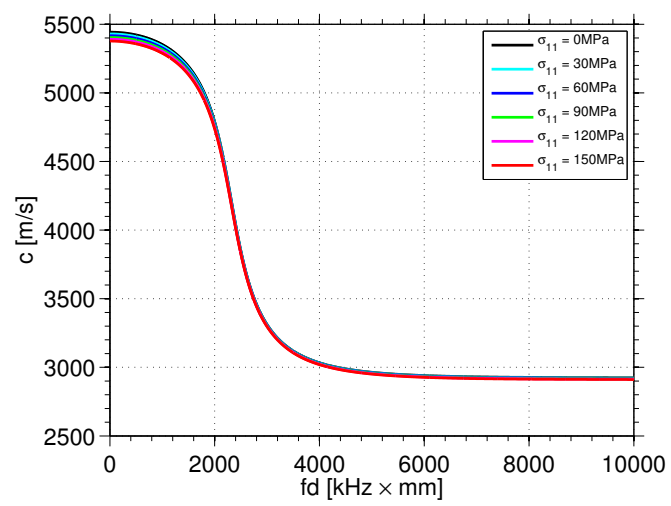

(a)

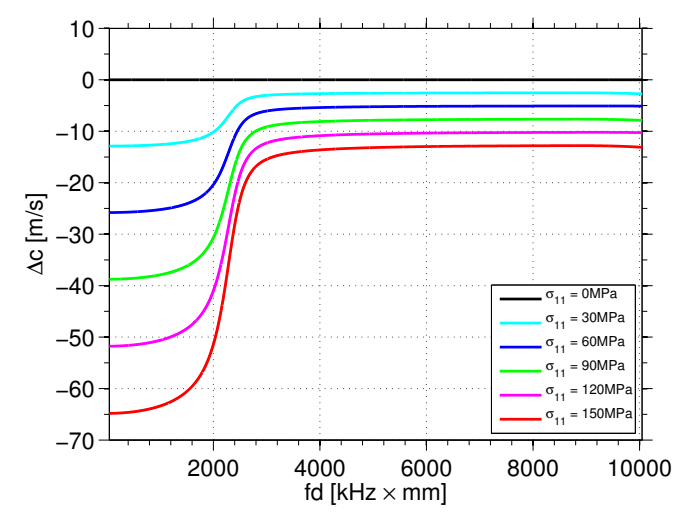

(b)

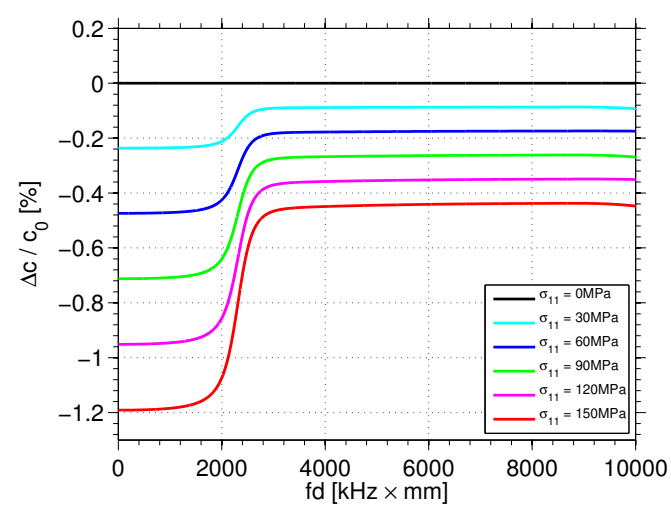

(c)

Figure 5.4: Acoustoelastic phase velocity dispersion curves for $S 0$ at natural frame of reference under tensile stress. (a) Phase velocity dispersion curves. (b) Velocity difference. (c) Velocity relative difference.

The group velocity change is depicted in Fig. 5.5.a, as well as its difference 
and relative difference, Fig.5.5.b and 5.5.c, respectively. For low frequencies the group and phase differences exhibit the same differences, as this are a low dispersive region for this mode and thus the velocities should coincide. At the most dispersive region, around $2500 \mathrm{kHz} \times \mathrm{mm}$, the group velocity difference changes its sign.

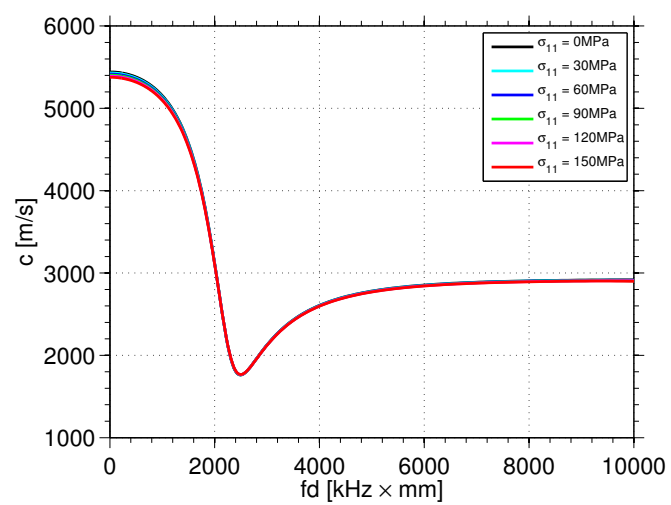

(a)

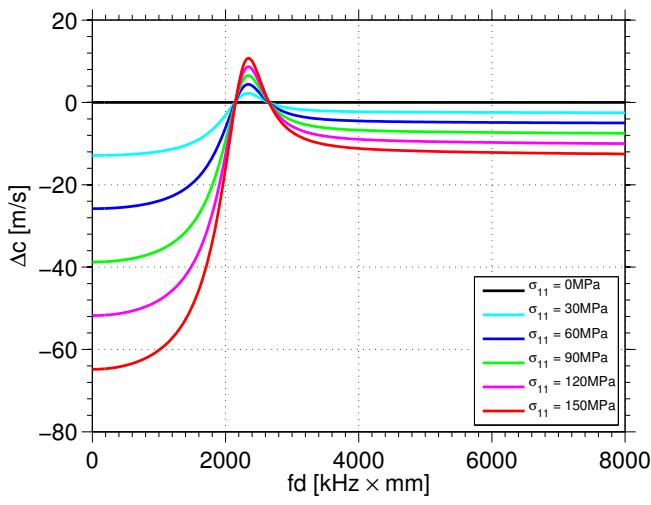

(b)

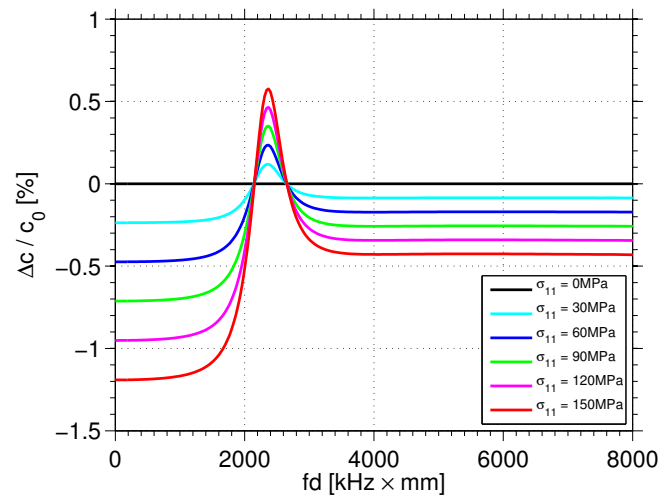

(c)

Figure 5.5: Acoustoelastic group velocity dispersion curves for $S 0$ at natural frame of reference under tensile stress. (a) Group velocity dispersion curves. (b) Velocity difference. (c) Velocity relative difference.

The sensitivity is shown in Fig. 5.6.a for phase velocity and 5.6.b for group velocity. Is is clear the linear behavior between velocities and stress as the curves essentially coincide for all the loads. By comparing Fig. 5.6.a to 5.3.b it is again clear that for the middle to high frequency range the phase velocity sensitivity of the $S 0$ mode is higher, in absolute value, than that for the $A 0$ mode. At high frequencies they converge to the same value of about $-3 \times 10^{-5} / \mathrm{MPa}$.

The group velocity sensitivity has the same value as the phase velocity for the low dispersion regions; at low and high frequencies, as expected. For the middle frequency, where dispersion is relevant, the sensitivities differ. The group velocity sensitivity is positive around $2500 \mathrm{kHz} \times \mathrm{mm}$. 


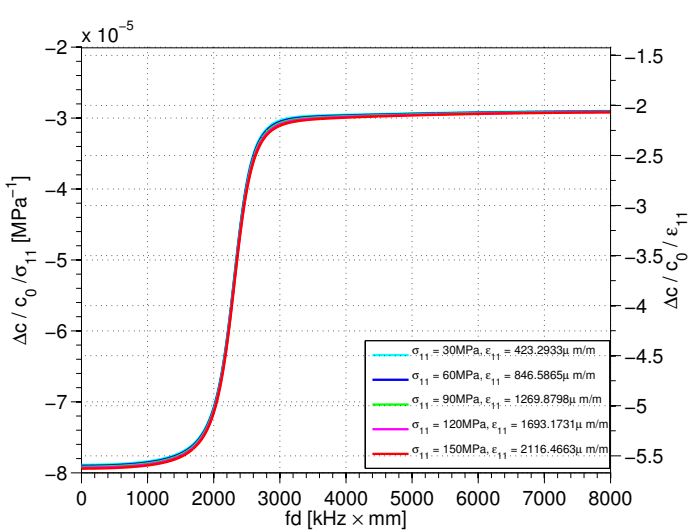

(a)

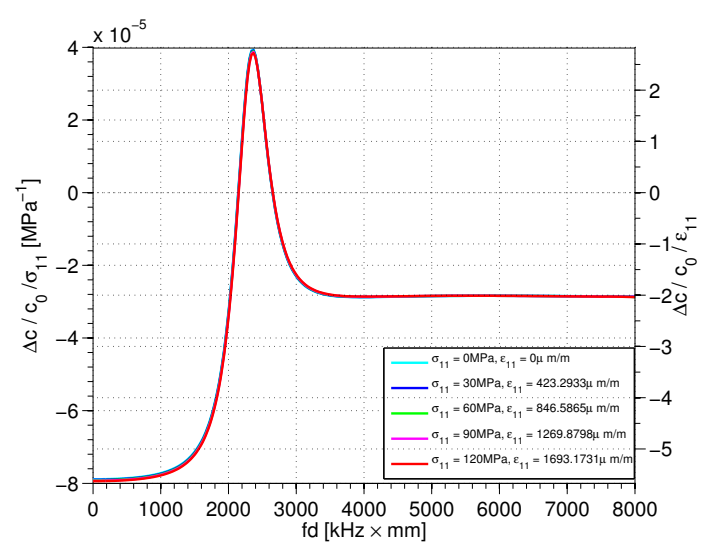

(b)

Figure 5.6: Acoustoelastic velocity sensitivity for $S 0$ mode at natural frame of reference under tensile stress. (a) Phase velocity sensitivity. (b) Group velocity sensitivity.

\subsection{3}

\section{Higher order modes}

The velocity change under stress was analyzed for the modes $A 1, S 1, A 2$ and $S 2$. Its results are briefly reported here. Analyzing the obtained dispersion curves it is concluded that, all these modes present linear relationship between velocity difference and stress. The phase and group velocity sensitivity curves for all the modes are shown in Fig. 5.7.

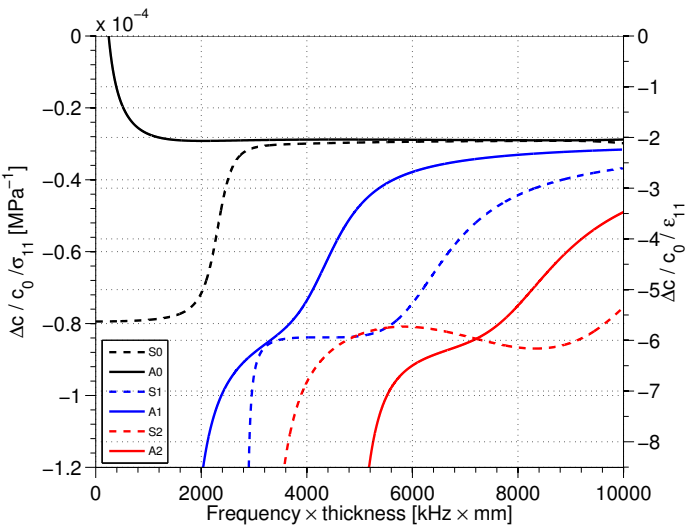

(a)

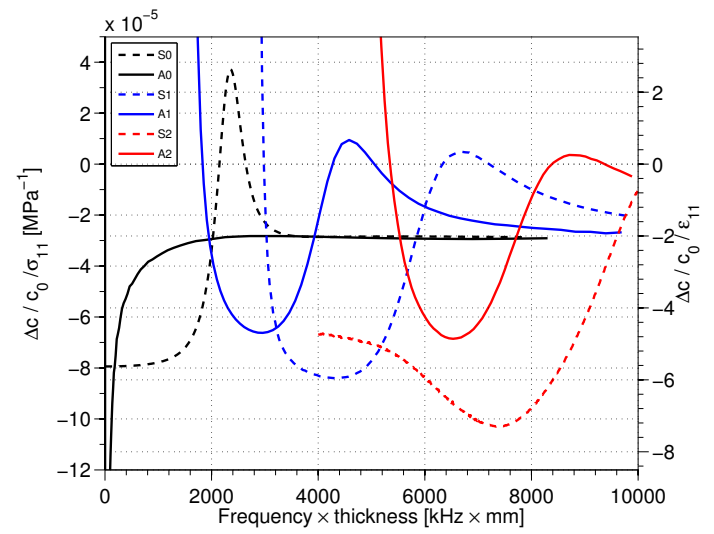

(b)

Figure 5.7: Acoustoelastic phase (a) and group (b) velocity sensitivity for up to second order modes at natural frame of reference.

As it can be seen, higher order modes always present negative sensitivity for phase velocity; the sensitivity is higher, in absolute value, than the fundamental ones, except for the $A 0$ mode at very low frequencies. Close to the cut-off frequency the sensitivity is elevated. Considering group velocity, as it happens for the $S 0$, there is also inversion of sign in the sensitivity for some 
regions. Other interesting characteristics is the highest sensitivity, in absolute value, for the regions where the group velocity has its peak (see Fig. 2.3.b).

\section{2}

\section{Initial reference frame}

The velocity variation under tensile stress was calculated with respect to the initial frame. In general, the curves present the same shape as those related to the natural frame of reference. Fig. 5.8 and 5.9 show the most significant aspects of $A 0$ mode phase and group velocities, respectively. In Fig. 5.8.a one can see that the same non-zero velocity for DC frequency present in the natural frame (Fig. 5.1.b) happens for the initial frame. Differences between them appears in the frequency where the zero crossing occurs, zoomed in Fig. 5.8.c; at the initial frame it is about $420 \mathrm{kHz} \times \mathrm{mm}$ whereas $240 \mathrm{kHz} \times \mathrm{mm}$ at the natural frame. The velocity at high frequency is lower at the initial frame than at the natural frame, as one can see in the Fig. 5.8.d.

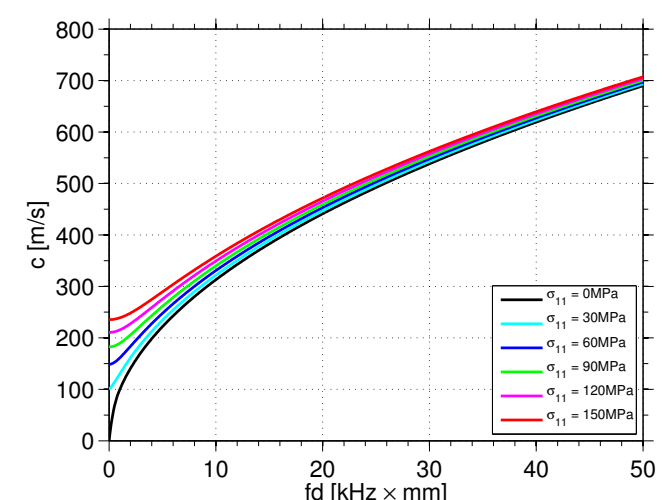

(a)

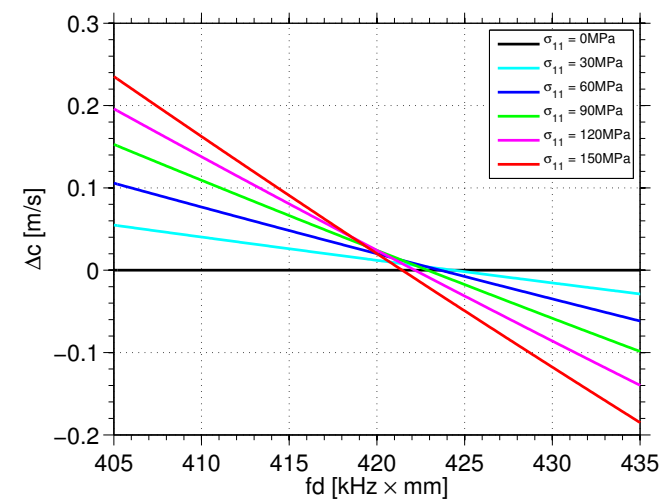

(c)

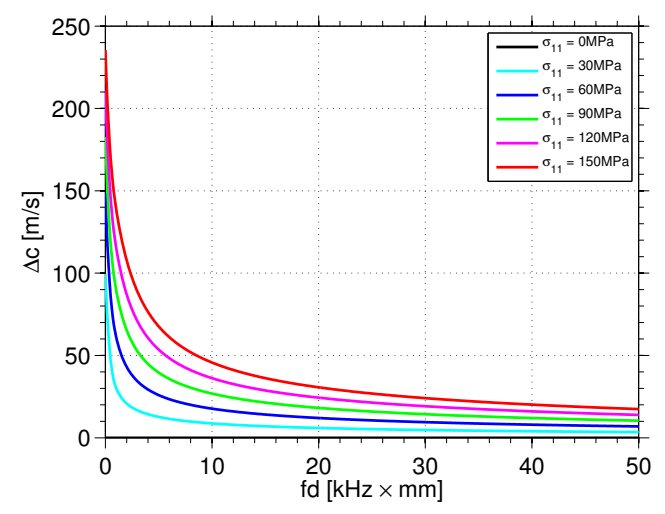

(b)

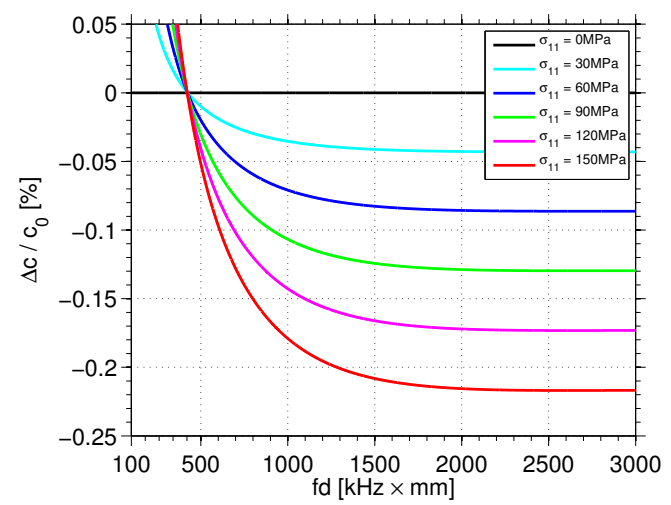

(d)

Figure 5.8: Acoustoelastic phase velocity dispersion curves for $A 0$ at initial frame of reference under tensile stress. (a) Low frequencies. (b) Velocity difference, low frequencies. (c) Velocity difference, zero crossing region. (d) Velocity difference, mid and high frequencies. 


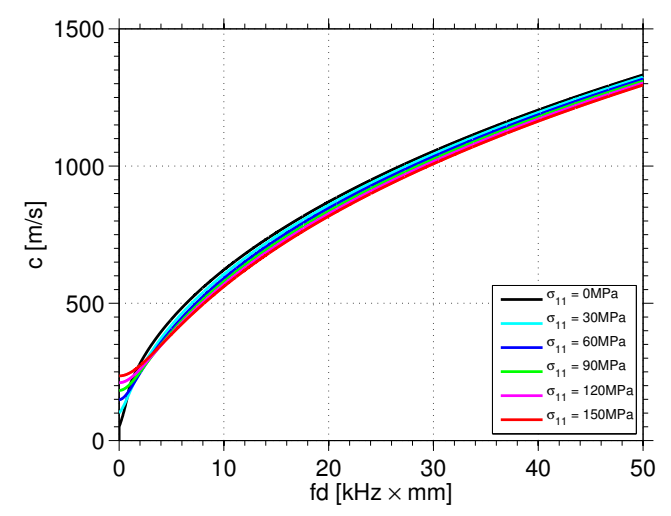

(a)

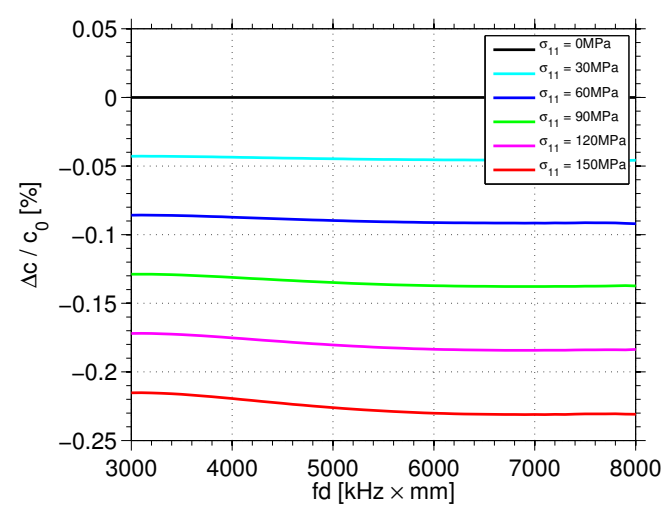

(b)

Figure 5.9: Acoustoelastic group velocity dispersion curves for $A 0$ at initial frame of reference under tensile stress. (a) Low frequencies. (b) Velocity relative difference, high frequencies.

The sensitivity at very low frequencies is as high as in the natural frame as the velocity DC value is the same. For higher frequencies the sensitivity, both in phase and group velocity, are lower compared to the natural frame. The sensitivities are shown in Fig.5.10.

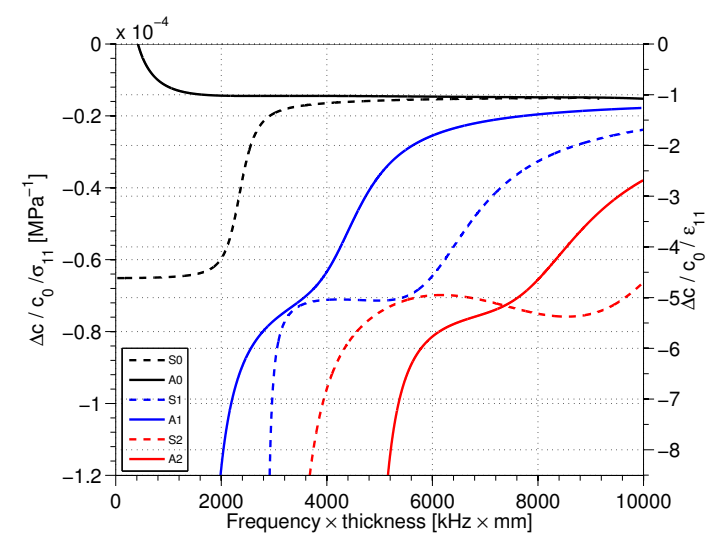

(a)

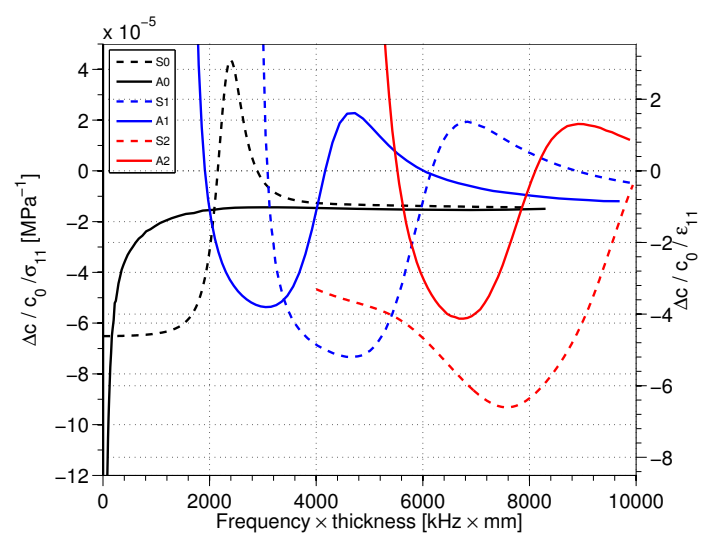

(b)

Figure 5.10: Acoustoelastic phase (a) and group (b) velocity sensitivity for up to second order modes at initial frame of reference.

In section 4.1.1 it was commented that discrepancies would arise if the original procedure proposed by Gandhi et al. [34] was used at the initial frame of reference. The most evident manifestation of this imprecision is at a very low frequency. The non-zero velocity at DC is not achieved if the original expression for the matrix $\mathbf{K}$ proposed by Gandhi is used instead of the correct one stated in (4.12). If the original expression is used, the behavior is like when EEC approximation is used, analyzed in the section 5.4 ahead.

Phase and group velocity relative difference for the $S 0$ mode are shown in Fig. 5.11, the sensitivities are shown in Fig. 5.10. The velocity difference 
and its sensitivity are lower than the natural frame.

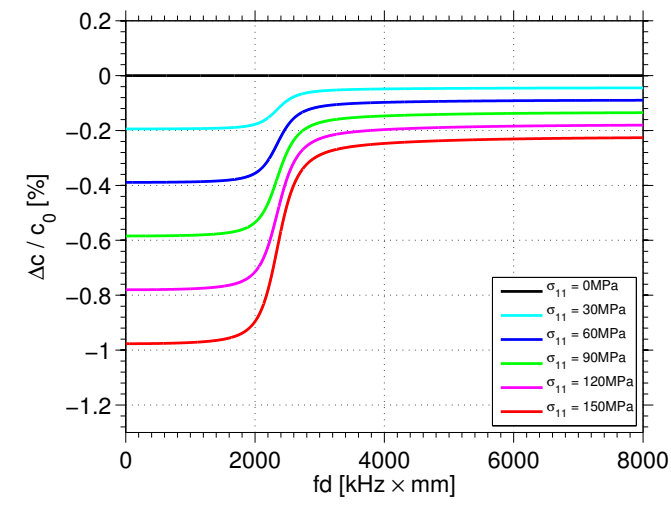

(a)

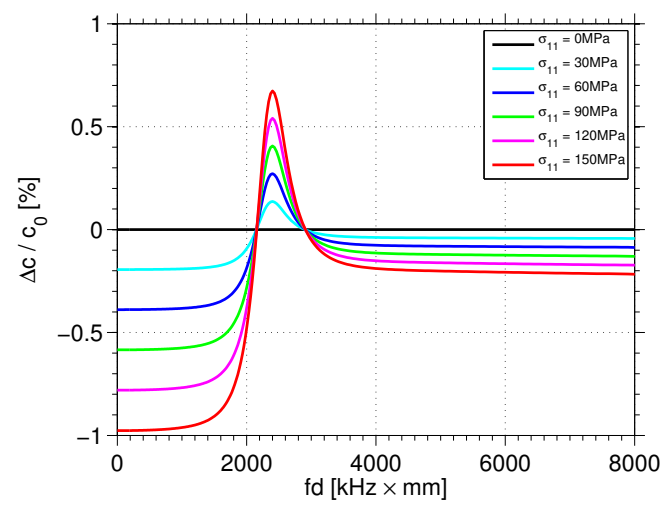

(b)

Figure 5.11: Acoustoelastic phase and group velocity relative difference for $S 0$ at initial frame of reference under tensile stress. (a) Phase velocity. (b) Group Velocity.

For the higher order modes it is analyzed only the sensitivity. The phase and group velocity sensitivity for all modes are shown in Fig. 5.10. As for the fundamental modes, sensitivity is less negative in the initial frame of reference. The regions where the group velocity is positive, is more positive than in the natural frame.

\section{3}

\section{Relationship between velocity at initial and natural frames}

At a first sight it seems that the velocity differences (given by (5.1) or (5.2)) related to the initial frame are a positively offset version of the curves at natural frame. This would make sense because in this particular case of uniaxial stress parallel to propagation direction, a longitudinal distance in the natural coordinate is shorter than the corresponding distance in the initial frame, thus, for the same propagation time, the velocity in the initial frame must be greater than the velocity in the natural frame.

In order to quantitatively analyze how the velocities at initial and natural frames are related, it is convenient to observe the velocities difference from the initial to the natural frame of reference. For the $A 0$ mode, this difference for phase and group velocities is shown in Fig. 5.12. For the $S 0$ mode the differences are shown in Fig. 5.13.

As reported in section 3.4 for bulk waves the difference from initial to natural wave velocity propagating along $x_{1}$ axes is given by (3.59). This relationship is not valid for guided waves because Lamb waves are composed by the contribution of several partial waves that propagate in the $x_{1}-x_{3}$ plane. Thus, in fact, one should use a combination of (3.59) and (3.60) where the 


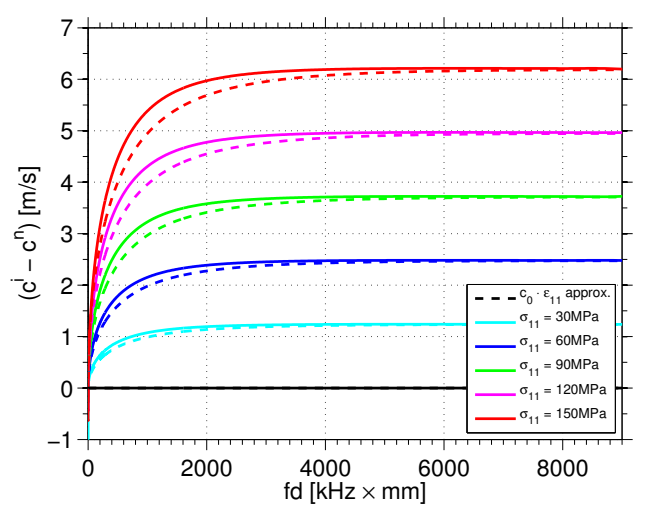

(a)

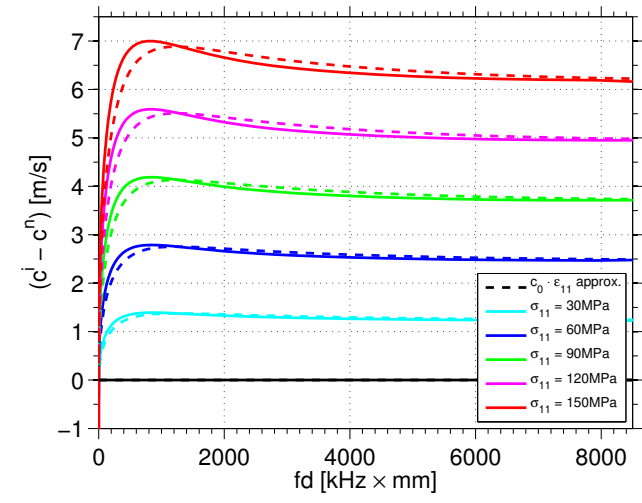

(b)

Figure 5.12: Acoustoelastic velocity difference from initial to natural frames for $A 0$. The curves color stand for the same loading as precedent figures, dashed lines represent the approximation given by (5.11). (a) Phase velocity difference. (b) Group velocity difference.

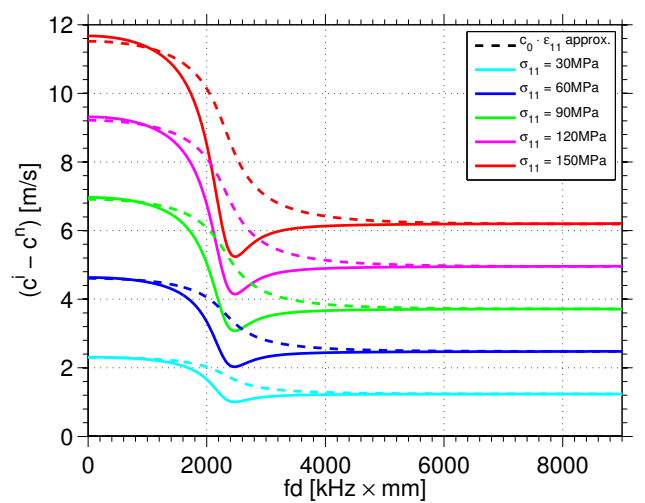

(a)

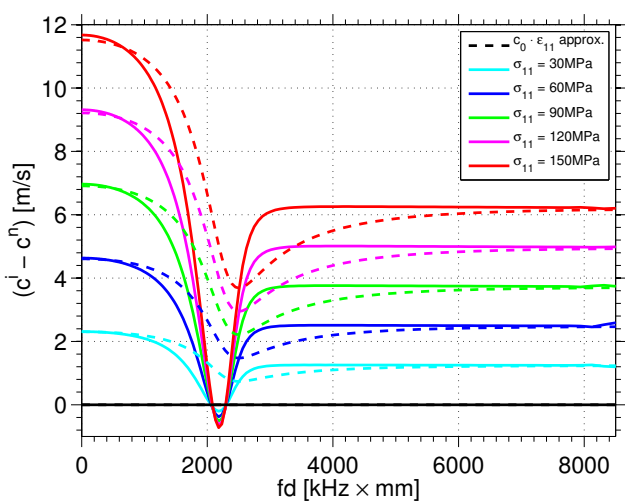

(b)

Figure 5.13: Acoustoelastic velocity difference from initial to natural frames for $S 0$. The curves color stand for the same loading as precedent figures, dashed lines represent the approximation given by (5.11). (a) Phase velocity difference. (b) Group velocity difference.

term related to the null stress velocity should be each partial wave that propagate in either $x_{1}$ or $x_{3}$ direction, respectively. In order to reach this exactly relationship one must know the partial waves propagation direction as a function of frequency. However, one can calculate a version of (3.59) for frequency dependent velocity, as Lamb waves, propagating along $x_{1}$. It would be

$$
c_{g r, p h}^{i}\left(f, \sigma_{11}\right)-c_{g r, p h}^{n}\left(f, \sigma_{11}\right)=c_{g r, p h}(f, 0) \varepsilon_{11},
$$

where the additional superscript $i$ or $n$ means that the velocity is related to the initial or natural frame, respectively. This is, however, an approximation. For non-dispersive regions, where the propagation behavior is close to a bulk wave, (5.11) may present an acceptable value. This comparison is shown in 
Fig.5.12 and 5.13 by the dashed line. It is observable that for low dispersive regions, i.e., high frequency for $A 0$ mode and low frequency for $S 0$ mode, the difference from initial to natural frames are very close to the approximation given by the dashed lines.

If the approximation introduced by (5.11) is applied into the sensitivity equation for initial frame (5.3), then the following is reached.

$$
\begin{aligned}
s_{g r, p h}^{i, \sigma}(f) & =\frac{\left[c_{g r, p h}^{i}\left(f, \sigma_{11}\right)-c_{g r, p h}(f, 0)\right] / c_{g r, p h}(f, 0)}{\sigma_{11}} \\
& \approx \frac{\left[c_{g r, p h}^{n}\left(f, \sigma_{11}\right)+c_{g r, p h}(f, 0) \varepsilon_{11}-c_{g r, p h}(f, 0)\right] / c_{g r, p h}(f, 0)}{\sigma_{11}} \\
& =\frac{\left[c_{g r, p h}^{n}\left(f, \sigma_{11}\right)-c_{g r, p h}(f, 0)\right] / c_{g r, p h}(f, 0)+\varepsilon_{11}}{\sigma_{11}} \\
& =\frac{\left[c_{g r, p h}^{n}\left(f, \sigma_{11}\right)-c_{g r, p h}(f, 0)\right] / c_{g r, p h}(f, 0)}{\sigma_{11}}+\frac{\varepsilon_{11}}{\sigma_{11}} \\
& =s_{g r, p h}^{n, \sigma}(f)+\frac{1}{E},
\end{aligned}
$$

and thus

$$
s_{g r, p h}^{i, \sigma}(f)-s_{g r, p h}^{n, \sigma}(f) \approx \frac{1}{E}
$$

The same derivation can be done for the strain sensitivities leading to

$$
s_{g r, p h}^{i, \varepsilon}(f)-s_{g r, p h}^{n, \varepsilon}(f) \approx 1
$$

The approximation for the sensitivities are plotted in Fig. 5.14 and 5.15 for the $A 0$ and $S 0$ modes respectively. Again, one may see that the approximation is valid for the low dispersion region, whereas the errors increase for the more dispersive parts, as predicted.

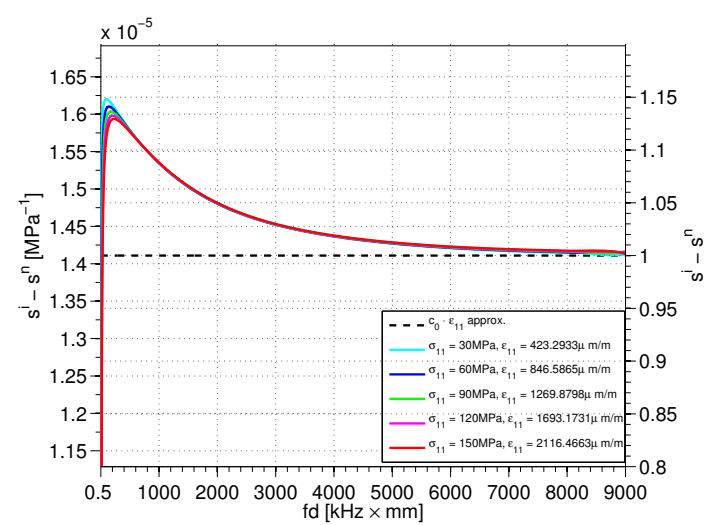

(a)

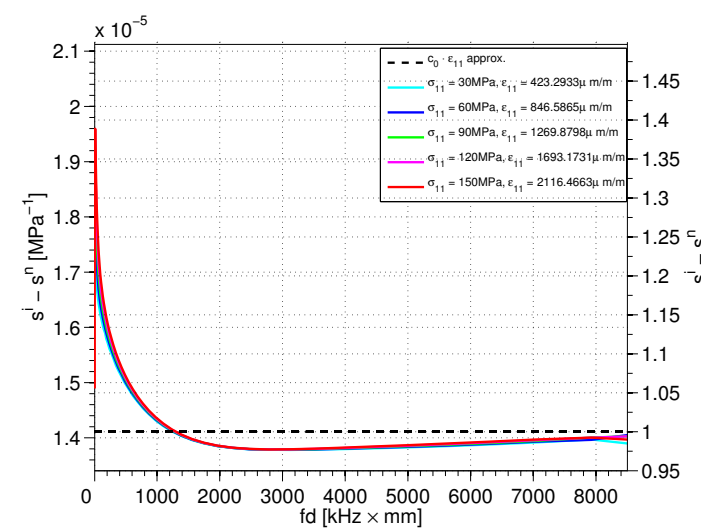

(b)

Figure 5.14: Acoustoelastic sensitivity difference from initial to natural frames for $A 0$. The curves color stand for the same loading as precedent figures, dashed line represent the approximation given by (5.13) or (5.14). (a) Phase velocity sensitivity difference. (b) Group velocity sensitivity difference. 


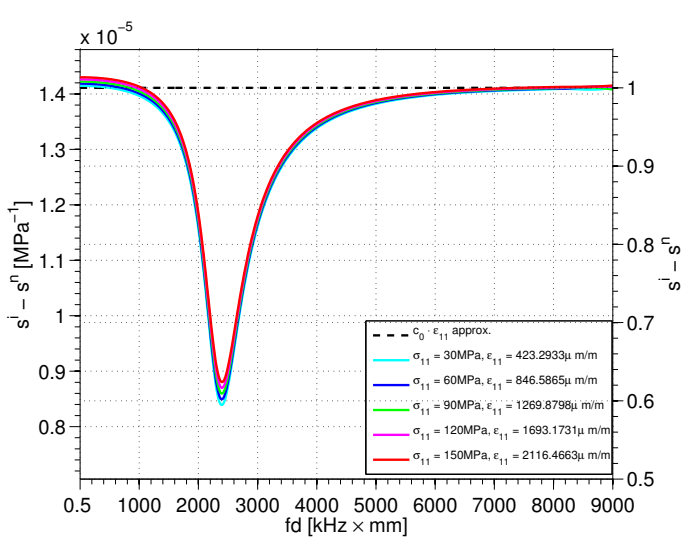

(a)

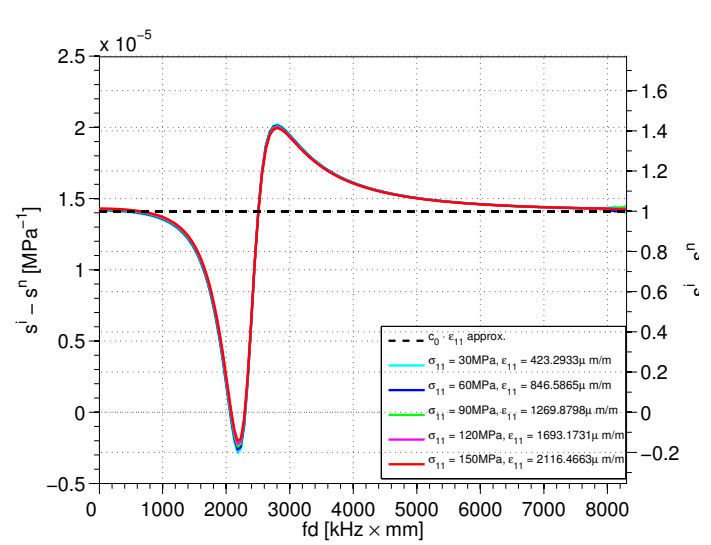

(b)

Figure 5.15: Acoustoelastic sensitivity difference from initial to natural frames for $S 0$. The curves color stand for the same loading as precedent figures, dashed line represent the approximation given by (5.13) or (5.14). (a) Phase velocity difference sensitivity. (b) Group velocity difference sensitivity.

\section{4}

\section{Effective Elastic Constants approximation}

In this section the dispersion curves for the acoustoelastic Lamb wave propagation are calculated using the approximation introduced by adopting the Effective Elastic Constants (EEC) into the partial wave technique procedure, as explained in section 4.4.1. The reason to analyze this approximation is to qualify the FEM simulation to be presented in chapter 6 . The material constants are the same as above. For axial loading the two EEC cases exposed in sections 4.4.1 are analyzed here.

\subsection{1}

\section{Approximation at natural reference frame}

The phase velocity dispersion curve for the $A 0$ mode for the EEC1 case is presented in Fig. 5.16. Comparing this last figure with Fig. 5.1 it can be seen that the use of the EEC cannot reproduce the complex behavior of the A0 mode under stress. Specially, as shown in Fig. 5.16.a and 5.16.b, the DC value for stressed medium is always null; the zero crossing is not present in the curves obtained by means of EEC and the velocity difference is negative for all the frequency range (see Fig. 5.16.c). At high frequency the behavior is close to the exactly solution, where the higher the stress the lower the velocity, suggesting that EEC approximation tend to the exactly value.

The group velocity present the same lack of precision, as shown in Fig. 5.17. There is no zero crossing (Fig. 5.17.a); the velocity difference is always negative (Fig. 5.17.b), and at high frequency the approximation appears to tend to the exactly solution. 


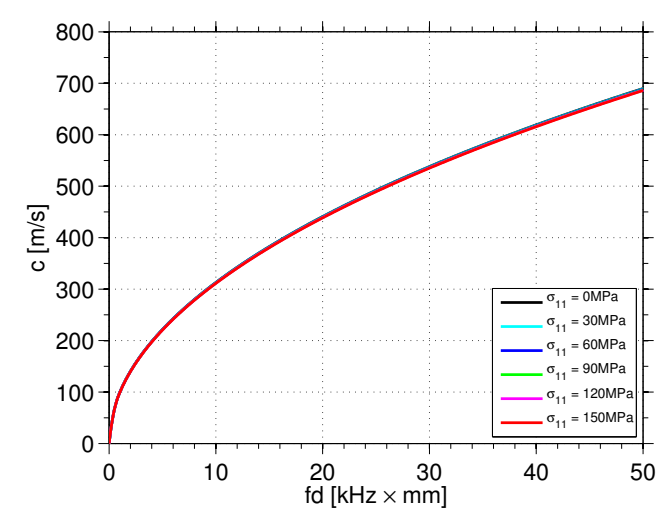

(a)

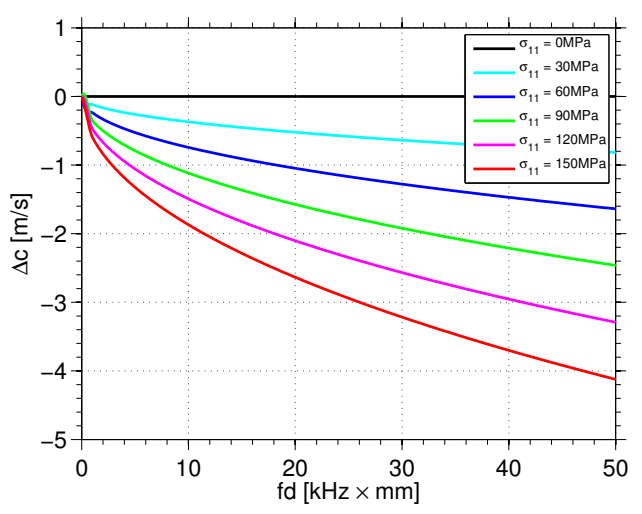

(b)

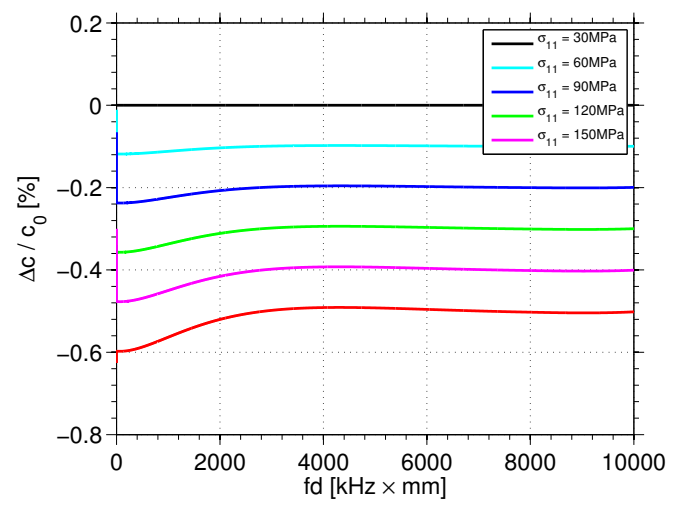

(c)

Figure 5.16: Acoustoelastic phase velocity dispersion curves for $A 0$ at natural frame of reference, EEC1 approximation under tensile stress. (a) Low frequencies. (d) Velocity difference at low frequency. (c) Velocity relative difference.

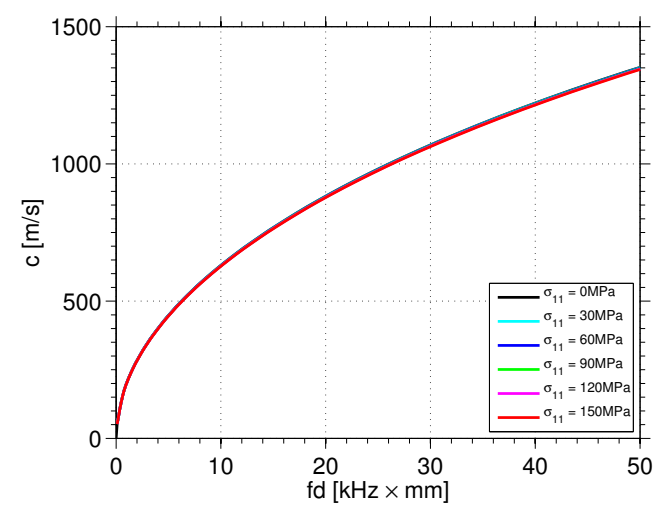

(a)

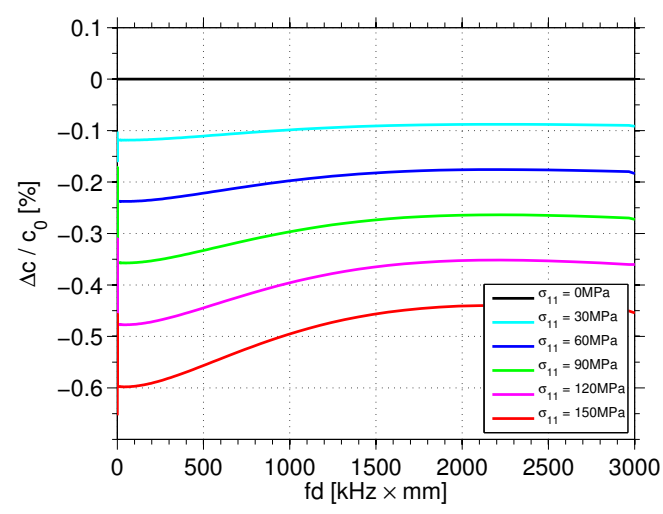

(b)

Figure 5.17: Acoustoelastic group velocity dispersion curves for $A 0$ at natural frame of reference, EEC1 approximation under tensile stress. (a) Low frequencies. (b) Velocity relative difference.

For the $S 0$ the use of EEC is, however, less precarious. Fig. 5.18.a shows the phase velocity relative difference from null stress obtained by EEC1 approximation, as it can be seen, the overall shape is the same as Fig. 5.4. 
For the group velocity (Fig. 5.18.b) a little discrepancy arises at the positives differences around $2500 \mathrm{kHz} \times \mathrm{mm}$, as it can be seen by comparing it with 5.5.c; the former presents lower values.

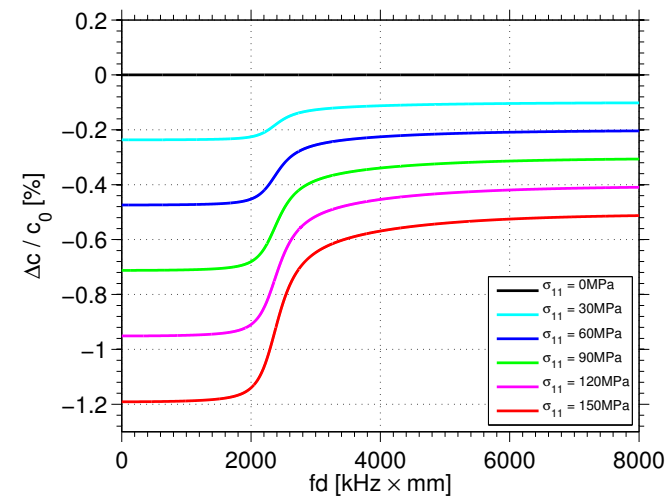

(a)

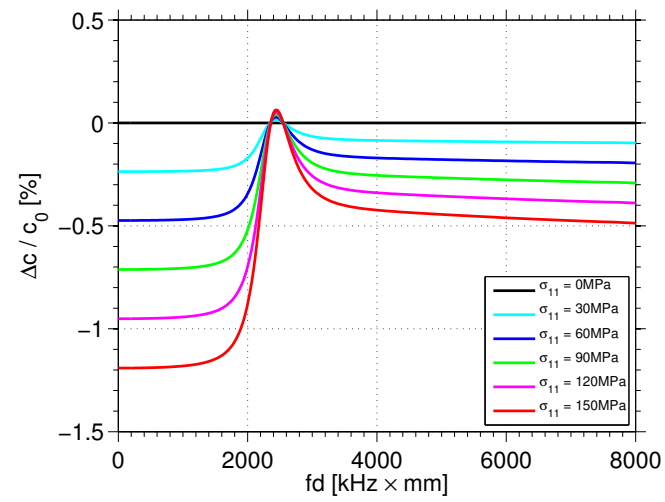

(b)

Figure 5.18: Acoustoelastic phase and group velocities relative difference from null stress for $S 0$ at natural frame of reference, EEC1 approximation. (a) Phase velocity. (b) Group difference.

It is then helpful to inspect the error introduced by the use of EECs. The relative error from the exactly solution and the one obtained by EEC is defined as

$$
\operatorname{error}^{E E C}(f, \sigma)=\frac{c_{p h, g r}(f, \sigma)-c_{p h, g r}^{E E C}(f, \sigma)}{c_{p h, g r}(f, \sigma)},
$$

where $c_{p h, g r}^{E E C}(f, \sigma)$ is the velocity obtained by the use of either EEC1 or EEC2.

The phase and group velocity errors for $A 0$ mode are shown in Fig. 5.19 where full lines stand for EEC1 and dashed lines for EEC2. Because EEC cannot reproduce the non-zero velocity at DC component, the error for low frequency is tremendous, ideally 100\%. Fig. 5.19.a shows the error in a logarithmic frequency axes making visible the values at quasi-DC frequency. The error diminishes as frequency rises. At higher frequency it is possible to distinguish the effect of using EEC1 or EEC2 (Fig. 5.19.b). It is notable that EEC1 is better than EEC2 for $A 0$ mode at natural frame of reference, because the error tend to decrease faster as frequency increases.

For the group velocity the errors are shown in Fig. 5.19.c and Fig. 5.19.d. The advantage of using EEC1 is also evident.

The errors for $S 0$ mode are shown in Fig. 5.20. The zero error frequency range is at low frequency, where there is low dispersion. As for the A0 mode, one can also conclude that for $S 0$ mode the EEC1 approximation is better. Error decrease faster from its peak value, between 2000 and $2500 \mathrm{kHz} \times \mathrm{mm}$ for both phase and group velocities, when using the EEC1 than EEC2. One can 


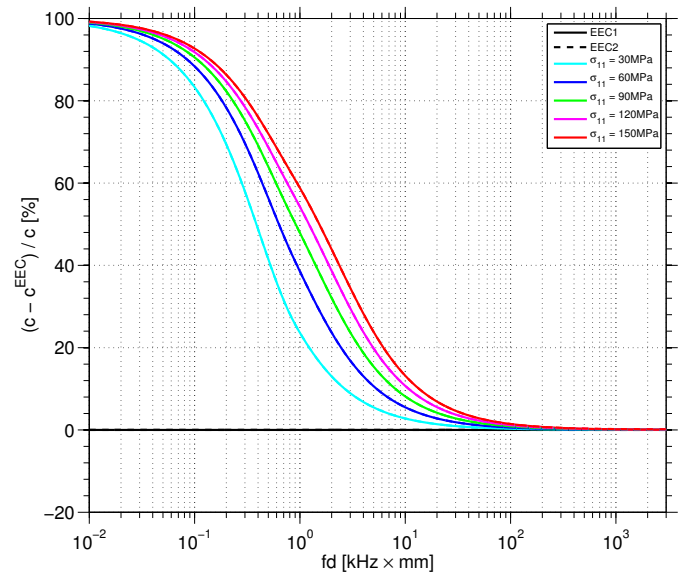

(a)

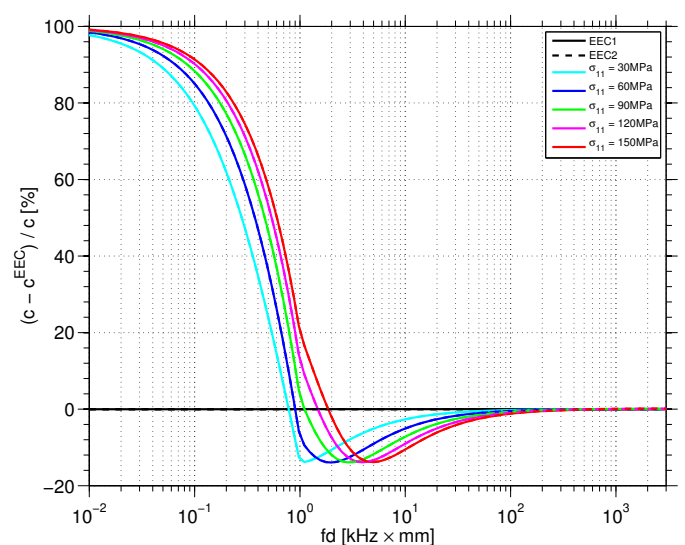

(c)

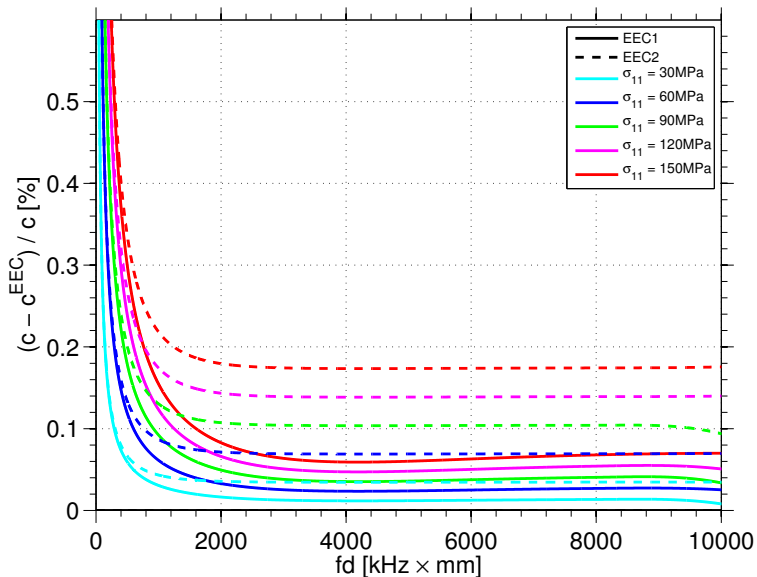

(b)

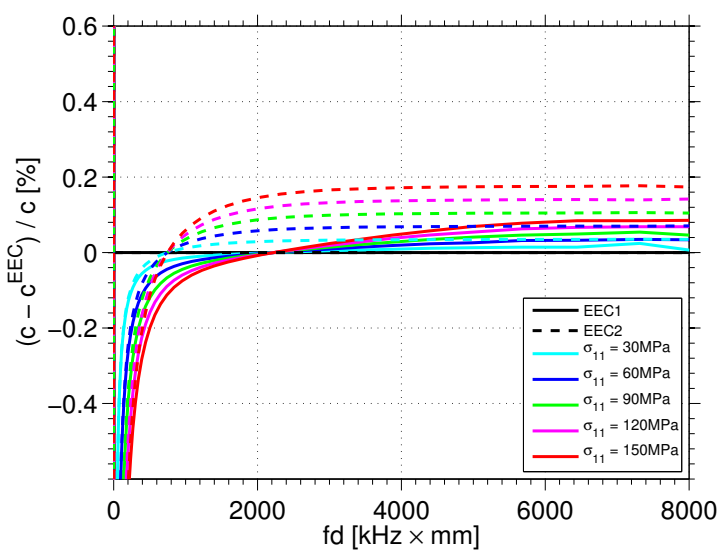

(d)

Figure 5.19: EEC velocity error for $A 0$ mode at natural frame of reference under tensile stress. Full lines stand for EEC1 and dashed lines for EEC2. (a) Phase velocity error in logarithmic frequency scale. (b) Phase velocity error Linear frequency scale. (c) Group velocity error in logarithmic frequency scale. (d) Group velocity error Linear frequency scale.

conclude that the errors are more significant at high dispersion regions; EEC1 case is better.

If one compares the order of magnitude of the errors with the velocity relative difference, in Fig. 5.1.h and 5.4.c, one can see that, despite of being absolutely small, the errors are not negligible compared to the velocity difference due to stress in the exactly solutions. This implies in sensitivity error, which can be quantized. Fig. 5.21 brings the error in the phase velocity sensitivity for fundamental, first and second order modes.

The only zero error region is for $S 0$ mode at low frequencies. For the fundamental modes the error is elevated in high dispersive regions; for low dispersive it tends to a constant value, about $16 \%$ for EEC1 and $40 \%$ for EEC2. The error for higher order modes vary considerably along the frequency 


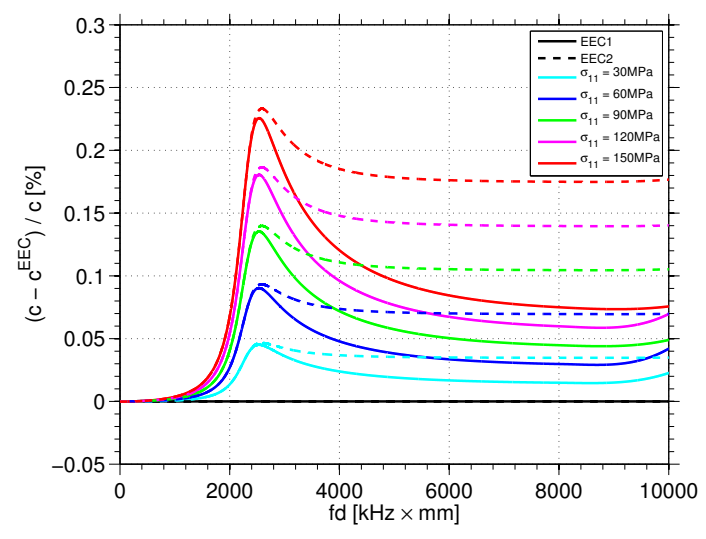

(a)

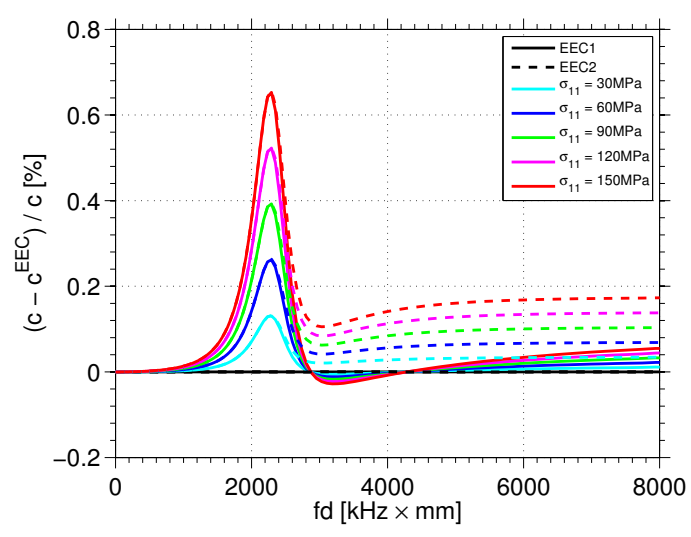

(b)

Figure 5.20: EEC velocity error for $S 0$ mode at natural frame of reference under tensile stress. Full lines stand for EEC1 and dashed lines for EEC2. (a) Phase velocity error. (b) Group velocity error.

range, due to the high dispersion of these modes. The order of magnitude of the EEC1 for these modes is about 10\%. For all modes the error using EEC1 is lower than by EEC2.

If one uses an equivalent anisotropic material to simulate the load effect this imprecision is present. The use of EEC is however quite convenient when using numerical approach as finite element or finite difference, specially for commercial software. This kind of numerical simulation is particular useful for complex structures (as non-regular cross section pieces, material with damages as cracks and notches, for instance) or if a multi-modal scenario is of interest, where there is different energy distribution among the modes and mode conversion. Thus one must be aware of the inherent imprecision in the use of EEC approximation. 


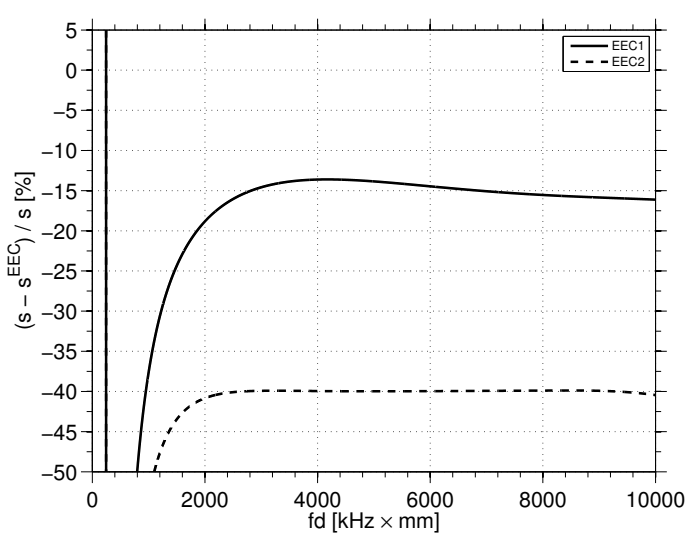

(a)

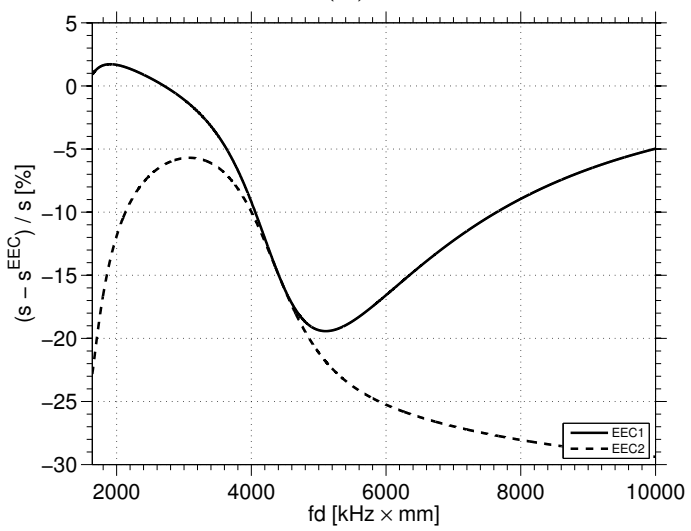

(c)

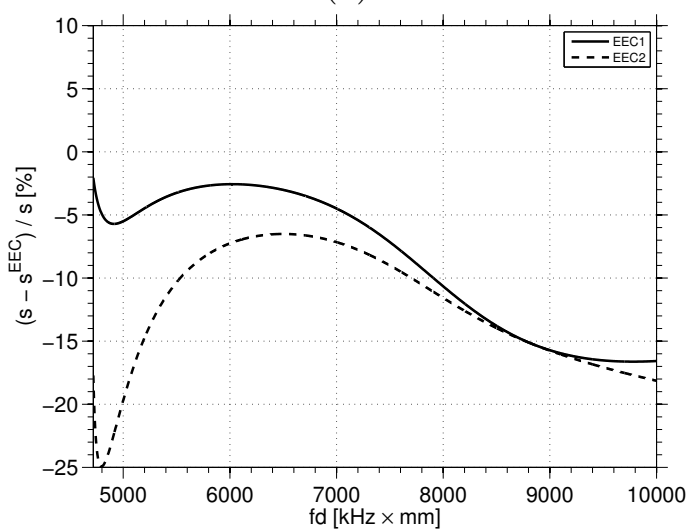

(e)

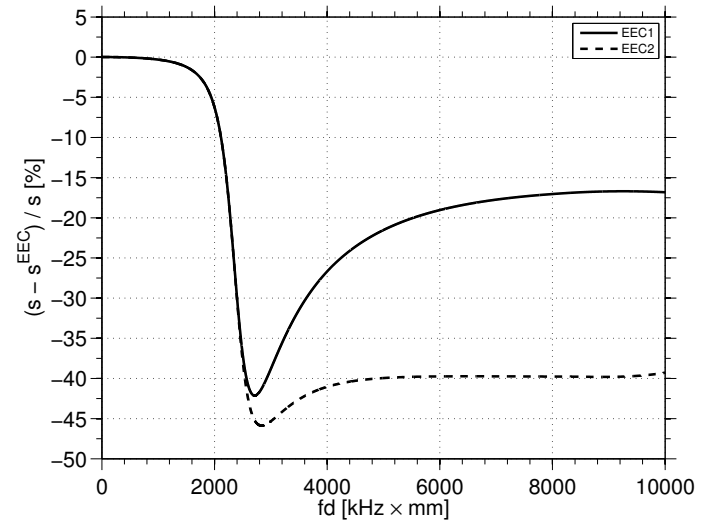

(b)

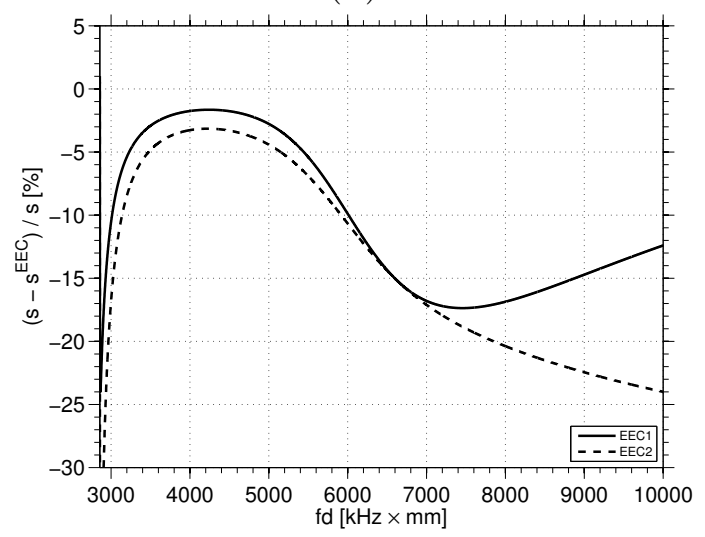

(d)

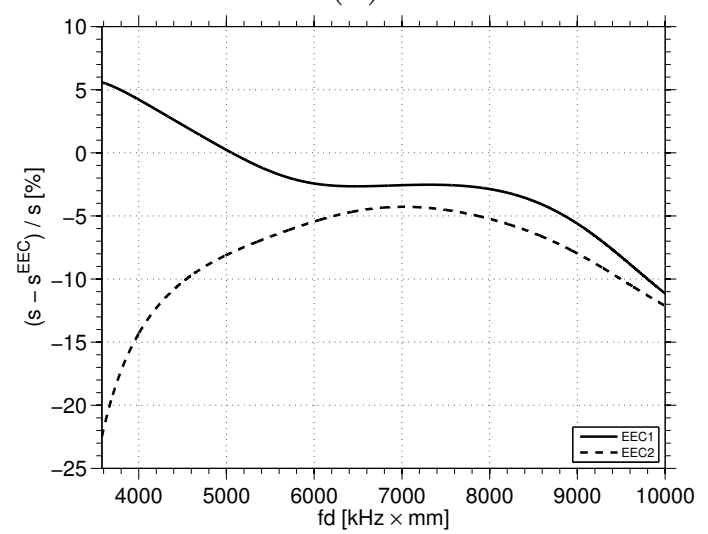

(f)

Figure 5.21: Sensitivity error for EEC1 and 2 at natural frame of reference. (a) A0 mode (b) S0 mode (c) A1 mode (d) S1 mode (e) A2 mode (f) S2 mode

\section{4 .2}

\section{Approximation at initial reference frame}

The same analysis was done for the initial frame of reference. Results are close to the natural case. The phase velocity sensitivity error is shown in Fig. 5.22. The error for the fundamental modes are much higher in either EEC1 and 2. For higher order modes the EEC2 shows in general lower error. 


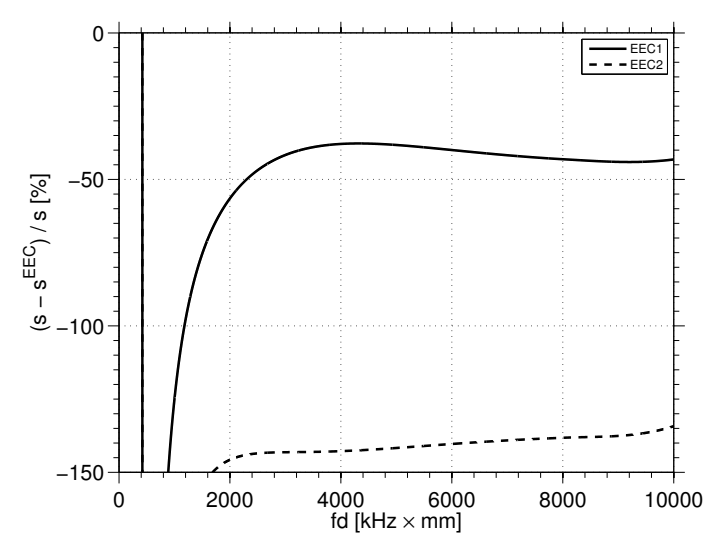

(a)

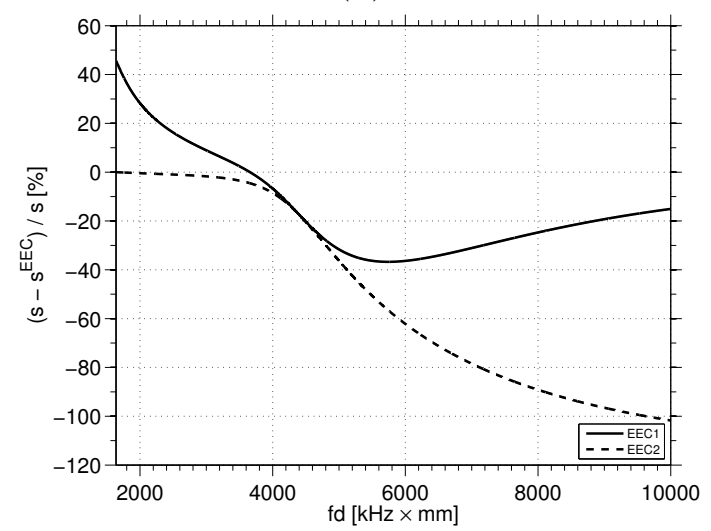

(c)

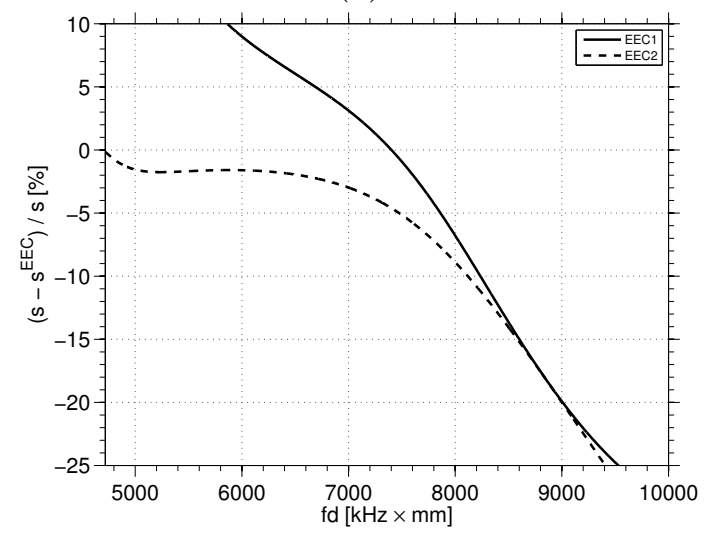

(e)

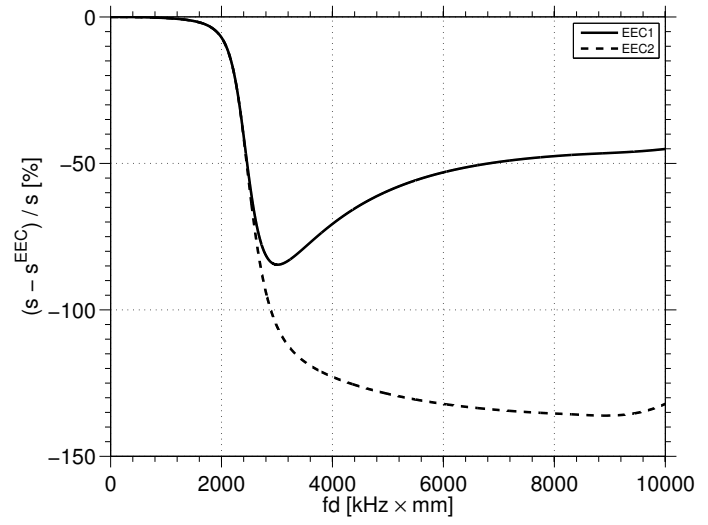

(b)

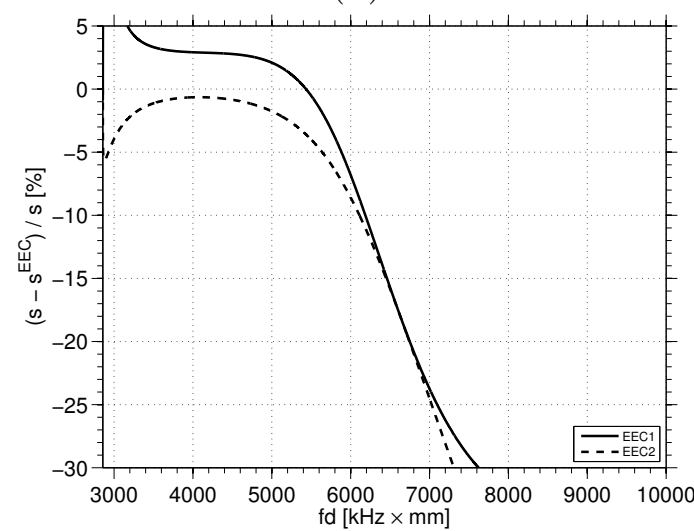

(d)

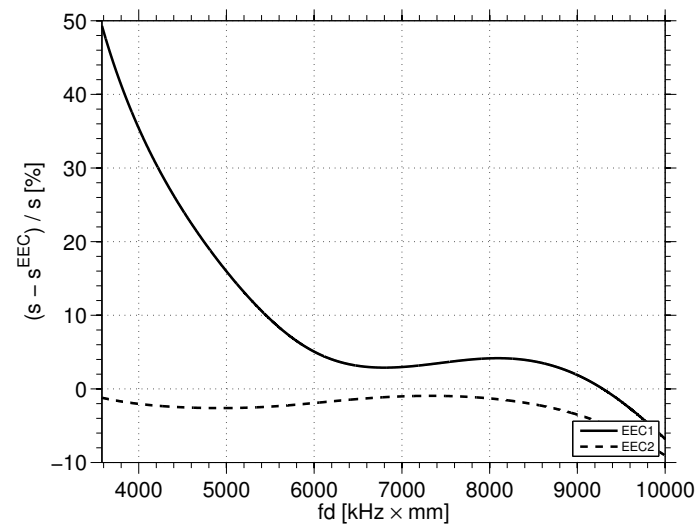

(f)

Figure 5.22: Sensitivity error for EEC1 and 2 at initial frame of reference. (a) A0 mode (b) S0 mode (c) A1 mode (d) S1 mode (e) A2 mode (f) S2 mode

\section{5}

\section{Review of literature results}

It is worth to confront the results presented above with other works found in the literature. Gandhi et al. [34] emphasized some aspects of $A 0$ and $S 0$ modes that are in perfect agreement with the ones brought here. This are the high differences at low frequencies, zero-crossing and low difference for high frequencies for the $A 0$ phase velocity; and the phase velocity difference 
behavior for the $S 0$ mode.

The fact that for low frequencies the phase velocity increases with tensile load but the group velocity decreases is presented by Loveday and Wilcox [99], despite they do not consider the complete accoustoelstic formulation, as they included finite strains but neglected the third order elastic constants (their work is based on the basic Mott's guided wave acoustoelastic theory $[120,121]$ ). The $A 0$ mode sensitivity at very low frequencies for phase and group velocities do agree with the work of Chen and Wilcox [33], which used the same acoustoelastic considerations as [99]. The most relevant aspects are the nonconstant sensitivity with stress (non-linear relationship of velocity variation with stress) for quasi-DC frequencies and the zero-crossing of the group velocity about $1 \mathrm{kHz} \times \mathrm{mm}$.

Interestingly in [33] it is stated that the only mode that presented nonnegligible velocity difference is the $A 0$ mode. As presented above, and on others works [34,107], the $S 0$, and higher modes, do present velocity difference, that depending on the frequency can be even greater than that for $A 0$. They did state that, because on their work only the very low frequency range is analyzed and, as seen above, at this short interval $A 0$ presents a huge velocity difference. However, the use of this frequency range may imply some hampering as this is an extremely dispersive regions (thus for conventional time difference measurement approach should imply in narrow band pulses which in turn imply in poor time localized signals). Moreover, the sensitivity is non-linear on stress.

Amjad et al. [107] experimentally investigated the $A 0$ and $S 0$ modes under tensile stress. They concluded the zero crossing in the $A 0$ mode about $200 \mathrm{kHz} \times \mathrm{mm}$ and the absence of zero-crossing in the velocity difference for $S 0$ mode.

It is not common that the papers precisely state in which frames the curves are calculated. However, following the development it is possible to infer it. Two very recent works on the literature calculated the dispersion curves at the initial frame of reference. Mohabuth et al. [102] used Ogden's acoustoelastic approach. Their work presented few qualitative results but the curve for the relative phase velocity difference at $S 0$ mode do agree with the one presented here revealing a $D C$ value of about $0.4 \%$ for $\sigma_{11}=60 M P a$. For the $A 0$ mode it is only possible to visualize the high change at very low frequencies and zerocrossing for phase velocity. Pau and Lanza di Scalea [101,122] also calculated the dispersion curves at initial frame of reference by deriving the equations of motion from the finite stress tensor. They highlighted the fact that the higher the tensile stress the lower the phase velocity, but do not commented 
on the different behavior of $A 0$ at low frequencies; they concluded also the linear relationship between velocity variation and stress; and that the group velocity difference may presents sign variation along the frequency range, as happens for the $S 0$ mode (see Fig.5.11.b). A numerical value reported on that work is about $-4 /(\mu \mathrm{m} / \mathrm{m})$ of sensitivity at $1000 \mathrm{kHz} \times \mathrm{mm}$ for, probably, the $S 0$ mode. This fits well with the sensitivity presented in Fig. 5.10.b.

There are very few works analyzing higher order modes acoustoelasticity. Pau and Scalea [101] calculated the acoustoelastic effect for many modes but in its absolute value instead of difference or sensitivity rendering interpretation not straightforward. A general characteristic is negative phase velocity sensitivity for all higher modes, sign inversion for group velocity sensitivity and it was commented the fact that where group velocity is maximum as it is its sensitivity. Gandhi et al. [34] calculated the absolute phase velocity difference for the first order modes but not the sensitivity; as far as it can be compared, the results match.

There is just one work [96] comparing the use of EEC with the exactly solution in the natural frame. However, this is not done in a systematic way as no velocity difference along the frequency range nor sensitivity were calculated. Results were shown by superposed curves, which hides quantitative conclusions. It was commented that EEC1 showed better results what agrees with the present results. 


\section{6}

\section{Lamb waves simulation}

Finite Element Method (FEM) is a useful tool for ultrasonic waves propagation analysis $[123,124]$, and specially for guided wave analysis [15, 28]. For simple models, such as a plates and also rods and cylinders, there are well-known theoretical equations that provide the necessary information. For instance, the dispersion curve. However, for more complicated geometries or non-homogenous media, solution may be only feasible by numerical methods, as FEM. Other situation where FEM is very convenient, and highly concerns NDE, is when geometries with defects, such as cracks, notches or delaminations, are modeled. FEM is also handy for simulations where there are several simultaneous modes; this is analyzed in chapter 8 ahead.

The acoustoelastic effect imply in a non-linear relationship between stress and strain, as states (3.5). Thus it is a priori impossible to simulate it by the linear relationship between stress and strain used by the linear elasticity theory adopted in the FEM analysis of commercial softwares. An alternative is to use an equivalent anisotropic medium by means of EEC [30]. There are few works simulating the acoustoelastic effect on Lamb waves. Chen and Wilcox [33] and Ferrari [31] used this but not on the full acoustoelastic framework, i.e., neglecting third order elastic constants. The other possibility is non-commercial FEM codes, as the Semi Analytical Finite Element (SAFE), $[32,99,100]$ where the element stiffness matrix is modified in order to include its dependence on the stress. Also the works that used SAFE for acoustoelasticity did not address the phenomenon in the complete framework.

In order to analyze the wave propagation in the time domain, dynamic transient analysis [125] is convenient. Temporal and spatial resolution are important concerns in FEM simulation. If resolutions are improperly chosen then the convergence of results may fail. The spatial resolution (i.e., the distance between nodes) must be chosen ensuring that waves can be spatially resolved. That is, a theoretical minimum node distance $(l e)$ is determined by Nyquist criterion, i.e., $l e=\frac{\lambda_{\min }}{2}$ [64]. It is, however, not recommended to use such a low resolution (long node distance) and it is often established rules of thumb for the maximum distance as 


$$
l_{e} \leq \frac{\lambda_{\min }}{N}
$$

where it is recommended that $N$ be at least 8 to 10 [124], 20 [28] up to 50 [15].

The time step of a transient simulation must be less than the ratio of the minimum distance between nodes and the fastest wave speed [15,124], thus the minimum sampling rate should be

$$
f_{s} \geq \frac{l e_{\min }}{c_{\max }}
$$

It has also been recommended in [28] that the sampling frequency should be greater than 20 times the highest frequency of interest.

It is used the ANSYS ${ }^{\circledR}$ Academic Research, Release $12.1[125,126]$ in this work. A two-dimensional $3 \mathrm{~mm}$ thick plate was modeled (in $x-y$ plane), as shown Fig. 6.1, using the plane element PLANE182 (Ref. [127] sec. 4, [128]) in plane-strain mode, because the plate on the transverse coordinate $(z)$ is infinite. It is worth noticing that the simulation in ANSYS ${ }^{\circledR}$ for two dimensional problems uses the $x-y$ plane. Despite the fact that all the development in the previous chapters has been done for waves propagating in the $x-z$ plane, in a plate infinite in $z$ direction, the coordinates $y$ and $z$ can be interchanged (on the input stiffness data and on the obtained results) without loss of generality.

It was used the Anisotropic Elastic Material Model (option ANEL in Ref. [127] sec. 2.5.7) where the material is specified by the whole stiffness matrix, in order to enable anisotropy when using EEC for a pre-stressed medium. The input for ANEL option are the 21 constants of the stiffness tensor. It is important to highlight that the order the stiffness matrix entries are input in ANSYS ${ }^{\circledR}$ is not the one used in Voigt notation. Data must be input according to Table 6.1 instead of Table 4.1. Note that index 4, 5 and 6 are permuted in Ansys notation related to the Voigt notation.

Table 6.1: Ansys notation for stiffness matrix.

\begin{tabular}{c|c}
$i j$ or $k l$ & $m$ or $n$ \\
\hline 11 & 1 \\
22 & 2 \\
33 & 3 \\
12 or 21 & 4 \\
23 or 32 & 5 \\
13 or 31 & 6
\end{tabular}

The material constants are the same given in Table 3.2. The stiffness tensor is calculated by (2.5) for a unloaded body or by (3.36) for stressed medium in the natural frame of reference and by (3.41) in the initial frame of reference. Then, the 21 constants are obtain consulting of Table 6.1. 


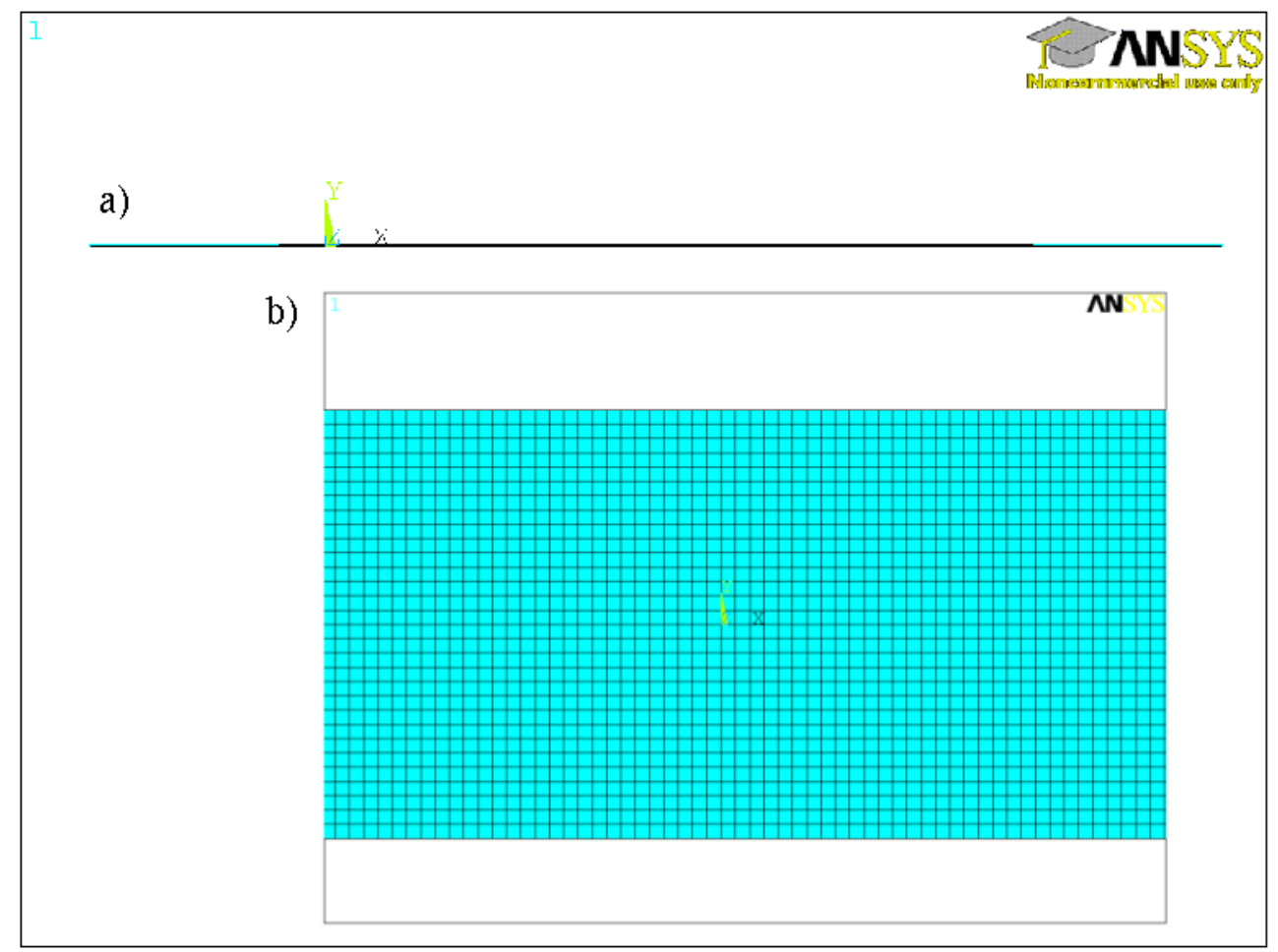

Figure 6.1: Example of modeled geometry. (a) Modeled plate plate, $3 \mathrm{~mm}$ thick $800 \mathrm{~mm}$ long $(x=[-50 ;+750] \mathrm{mm}$ and $y=[-1.5 ; 1.5] \mathrm{mm})$. The blue regions at the extremity of the plate are the absorbers $(200 \mathrm{~mm}$ long each, left end: $x=[-250 ;-50] \mathrm{mm}$, right end $x=[+750 ;+950] \mathrm{mm})$. (b) Mesh zoom.

Wave excitation was done by imposing a time-dependent out-of-plane displacement $\left(u_{y}\right)$ on the surface node at position $(x=0)$. Mode selection can be made in the excitation step by imposing simultaneously displacement in both surfaces in the same longitudinal position [15]. In other words, by applying the exactly same excitation at coordinates $(x=0, y=+h)$ and $(x=0, y=-h)$, where $h=d / 2$ is half of the plate thickness, then only antisymmmetrical modes are excited, for instance the A0 mode. On the contrary if it is applied the negative pattern on the opposite surfaces, $+u_{y}$ at $(x=0, y=+h)$ and $-u_{y}$ at $(x=0, y=-h)$, then the symmetrical modes are excited, for instance the S0 mode. If excitations is applied only at one surface, either top or bottom, then both types of modes propagate.

If a wave front hits the plate end, then reflection occurs. In order to avoid it, there are some subterfuges. The simplest is to model the medium long enough so that, for the propagating wave velocity, reflections are not sensed at the position of interest for the chosen time window. This approach may imply in big models and enormous computation effort. The other is to model absorbers at the ends. There are some ways to do this [129-139]. The 
methodology used herein is the one presented by Shen and Giurgiutiu in their very recent work [139], which is a modification of the well-known LysmerKuhlemeyer method from 1969 [129] for better absorption of Lamb waves.

The original Lysmer-Kuhlemeyer method uses spring-damper element (as COMBIN14 element see Ref. [127] sec. 4) at the end in which absorption is wanted, either in longitudinal and transverse direction. Spring constant is nullified and the damper constants is set proportional to the longitudinal and shear velocities respectively, in order to match the incident energy [135]. This implementation is commonly named Lysmer-Kuhlemeyer non-reflective boundary (LK-NRB).

LK-NRB is not effective for Lamb mode because these are formed by the interference and superposition of many partial longitudinal and transverse waves undergoing multiple reflections between the surfaces. Thus the simple LK-NRB implementation cannot totally absorb the incident Lamb wave modes. The new method proposed by Shen and Giurgiutiu, named as Modified Lysmer-Kuhlemeyer non-reflective boundary (MLK-NRB), implements dampers at the top and bottom surface along a certain distance at the plate's end, say $L_{a}$. In this absorber region the damper constant is increasing according to the function $f\left(x_{a}\right)$ where $x_{a}$ is the distance in the absorber. At the absorber end, $x=L_{a}$, dampers are included at the plate cross section, as the original technique. Fig. 6.2 schematizes the absorber.

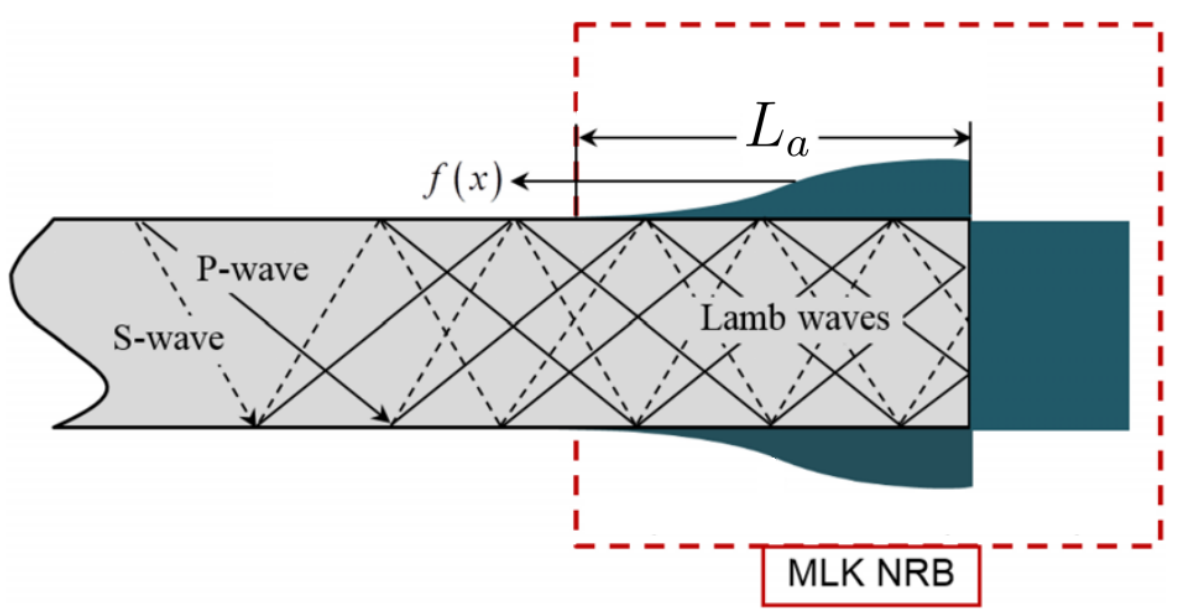

Figure 6.2: MLK-NRB absorber. Adapted from [129] 
The damping constant are then set to

$$
\begin{gathered}
C_{N}= \begin{cases}f\left(x_{a}\right) l_{e} \rho c_{L} & , \text { at the surfaces, } \\
l_{e} \rho c_{L} & , \text { at end section, }\end{cases} \\
C_{T}= \begin{cases}f\left(x_{a}\right) l_{e} \rho c_{T} & , \text { at the surfaces, } \\
l_{e} \rho c_{T} & , \text { at end section, }\end{cases}
\end{gathered}
$$

where $C_{N}$ and $C_{T}$ are the longitudinal and transverse damper constant, $l_{e}$ is the element size, $\rho$ the density, $c_{L}$ and $c_{T}$ the longitudinal and shear waves velocity, respectively, given by $c_{L}=\sqrt{C_{11} / \rho}, c_{T}=\sqrt{C_{44} / \rho}$, in which $C_{i j}$ is the material stiffness tensor.

The half Hanning window is used in this work as damping function, as recommended by Shen and Giurgiutiu. Its expression is

$$
f\left(x_{a}\right)=\frac{\delta}{2}\left[1-\cos \left(\pi \frac{x_{a}}{L_{a}}\right)\right], 0 \leq x_{a} \leq L_{a}
$$

where $\delta$ is a constant set to 0.3 . More details on the absorption implementation and performance are found in [139].

\section{1}

\section{Validation for unstressed medium}

Initially simulations for the unstressed medium were performed in order to validate the simulation procedure. Either the A0 and S0 mode were independently excited by the double imposed out-of-plane procedure exposed above. A broadband signal was used for excitation, such that the maximum frequency of the signal was set lower than the cut-off frequency of the first order modes, A1 and S1, respectively, in order to ensure propagation of just the fundamental modes. The response is the displacement and it is collected at every node on the surface.

\subsection{1}

\section{A0 mode}

For the A0 mode the excitation is a sinc-like signal where the DC component was filter-out. This was done because the low frequency region has low-velocity (see Fig. 5.2.a), thus a very long time window should be used in order allow observation of these components wave-fronts. This excitation signal is shown in Fig. 6.3, in both time and frequency (its Fourier transform) domains. It is a bandpass signal with $-6 d B$ bandwidth from $50 k H z$ up to 
$400 \mathrm{kHz}$. This frequency range excite the low-frequency and high dispersive region of this mode, up to $1200 \mathrm{kHz} \times \mathrm{mm}$ (see Fig. 5.1.a).
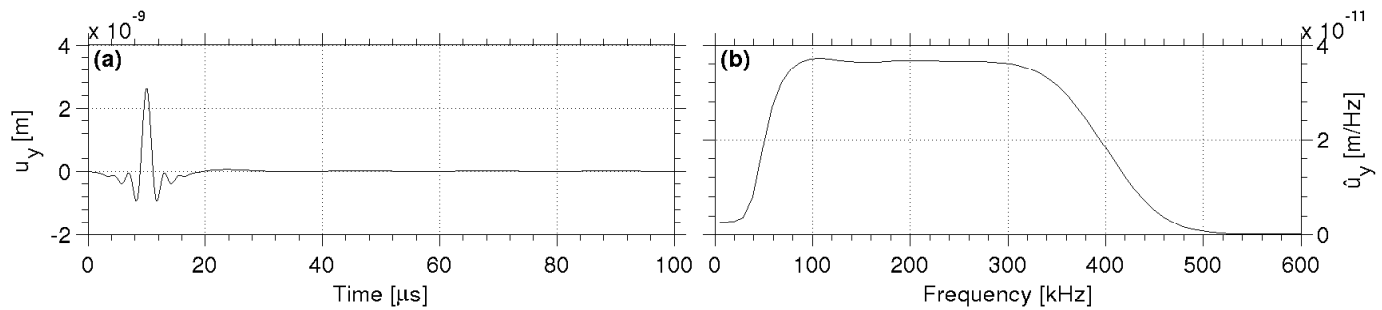

Figure 6.3: A0 excitation signal. (a) Signal in time domain. (b) Signal in frequency domain.

It is reported below the result of a simulation performed with sampling frequency of $40 \mathrm{MHz}$ and spatial resolution of $0.1 \mathrm{~mm}$, which is equivalent to about 50 times the minimum wavelength. The plate length in this simulation is $330 \mathrm{~mm}$. As the A0 modes has a predominant out-of-plane motion, it is convenient to observe the out-of-plane displacement $\left(u_{y}\right)$. Fig. 6.4 shows the propagated wave sensed as out-of-plane displacement at the top surface. In Fig. 6.4.a a grayscale map is used to plot the wave for every node at the surface, gray color means zero displacement, white means maximum positive displacement, whereas black means maximum negative displacement. One can see that the wavefront spread as it propagates, this happens due to dispersive behavior of the A0 mode. Dispersion is even more clear in Fig. 6.4.b, where time-traces for some surface positions are plotted versus time in a seismogramlike plot. It is clear that the sinc-like signal applied at the origin has it low frequency components spreads at greater time instants (low velocities) and high frequency at low time instants (high velocities), as is the behavior of the A0 mode dispersion curve (see Fig. 5.1).

From the sensed signal one can obtain the dispersion curve by the methods presented in section 2.3.1. The two dimension Fourier transform and the phase spectral analysis are tested here. As stated in section 2.3.1.1 the two-dimension Fourier transform of a wave signal represents the wavenumberfrequency pair of this wave. This is verified in Fig. 6.5.a, where it is shown the absolute value of the two-dimension Fourier transform of the A0 mode outof-plane displacement, in reverse grayscale map (maximum is black, minimum is white). The theoretical dispersion curve is superposed to the frequencywavenumber plane. As one can see, the theoretical curve seems to coincide with the maxima of the two-dimension Fourier transform. However, extracting the dispersion by taking the $(\kappa, f)$ points that are the maxima provides a curve 


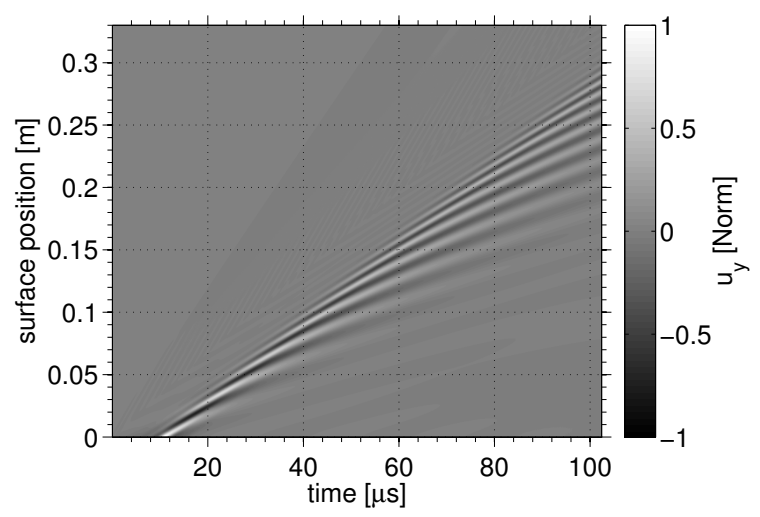

(a)

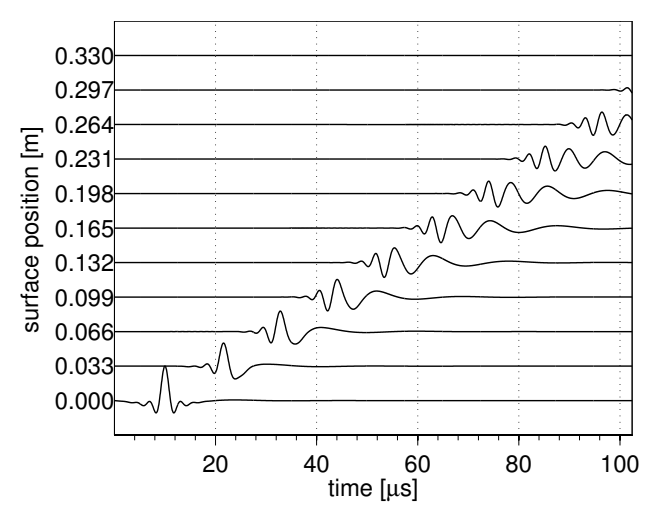

(b)

Figure 6.4: A0 propagation sensed as out-of-plane displacement at top surface. (a) Grayscale map. (b) Seismogram-like plot.

that is limited to the resolution of both, frequency and wavenumber. This can be verified in Fig. 6.5.b where the maxima were extracted.

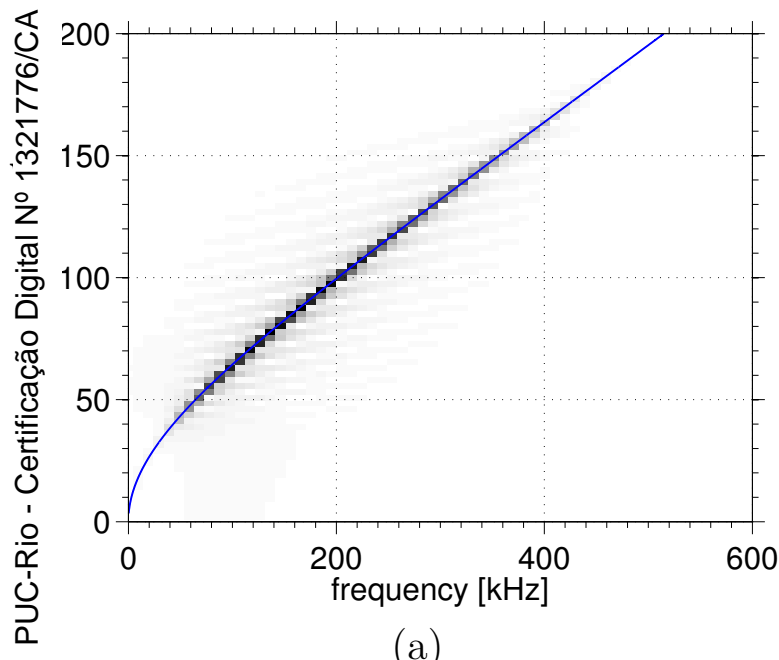

(a)

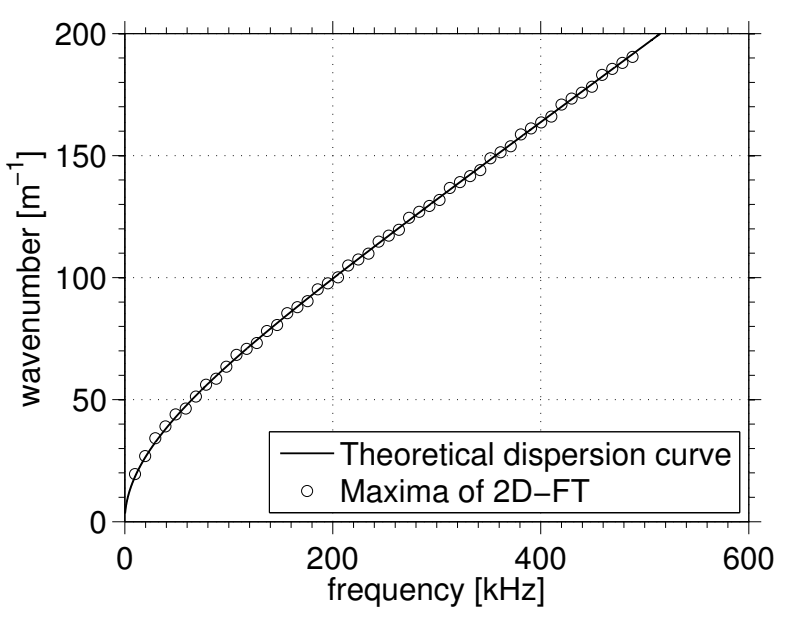

(b)

Figure 6.5: A0 dispersion extraction by two-dimension Fourier transform. (a) Two-dimension Fourier transform absolute value in reverse grayscale map and theoretical dispersion curve represented by the blue line. (b) Extracted dispersion curve.

Phase spectral analysis method explained in section 2.3.1 is performed. The wave form in two different position are necessary for extracting the phase velocity curve. In this case, it was used the signal at positions $x=0.0275 \mathrm{~m}$ and $x=0.0285 \mathrm{~m}$, as shown in Fig. 6.6.a for a time window from 10 up to $60 \mu s$. In this plot the signal time traces and their Fourier transform are reported. In the time-trace plot it is clear that the two waves are time shifted. The Fourier transform absolute value presents no difference because there is no attenuation in the simulation. Their phase show, however, considerable difference. The 
phase difference is used in (2.26) in order to obtain the phase velocity, which is plotted in Fig. 6.6.b. In this last figure the theoretical phase velocity curve, calculated by means of the theory explained in section 5 is confronted with the extracted curve. It is clear that they match. This procedure provide more precise values than the two-dimension Fourier transform.
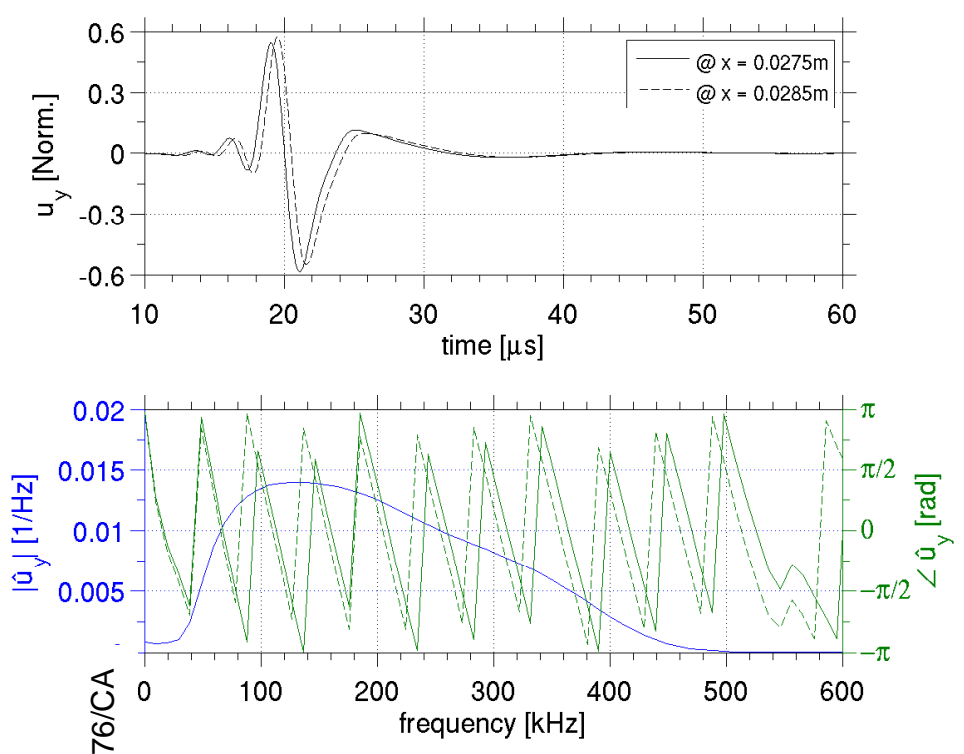

(a)

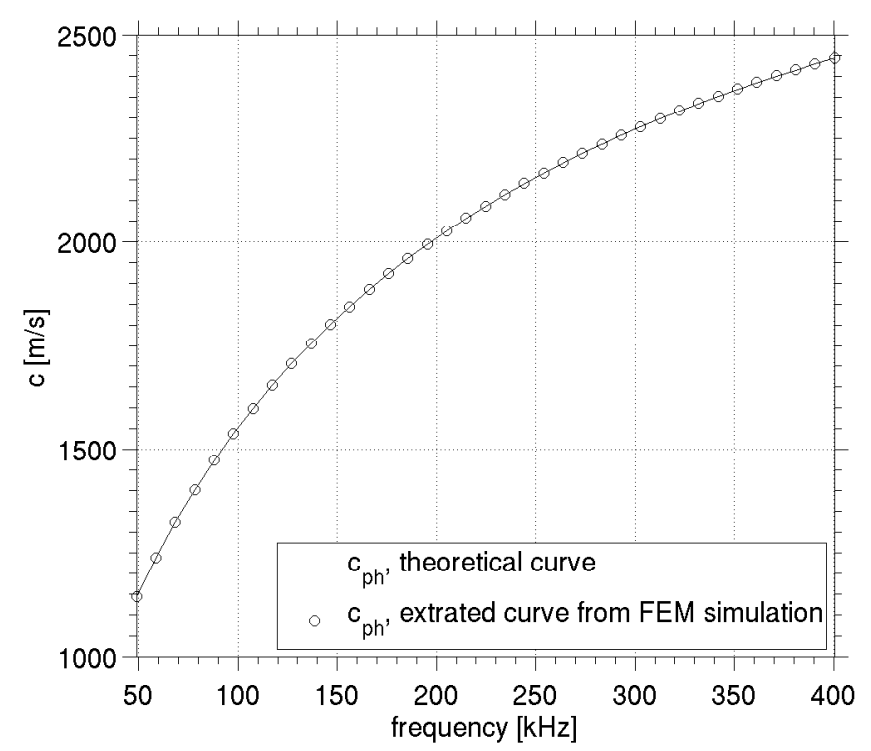

(b)

Figure 6.6: A0 dispersion extraction by phase spectral analysis. (a) Signal at two surface positions, in time domain, top, and their Fourier transform, bottom, in both amplitude, blue lines, and phase, green lines. (b) Extracted phase velocity dispersion curve.

In order to ensure that the FEM geometrical and temporal parameters provide accurate enough results, the simulation and dispersion curve extraction by the phase spectral analysis procedure was repeated for some resolutions. The absolute root-mean-square error (rmse) of the extracted phase velocity, related to the theoretical one, and the normalized root-mean-square error per mean velocity was used to judge the model goodness. These values are reported in Table 6.2. It is also reported in the table the sampling frequency to the minimum propagation time between nodes ratio $\left(f_{s} /\left(c_{\max } / l_{e}\right)\right.$, the sampling frequency to the maximum frequency ratio $\left(f_{s} / f_{\max }\right)$, thickness to nodes distance ratio $\left(d / l_{e}\right)$, and minimum wavelength to nodes distance ratio $\left(\lambda_{\min } / l_{e}\right)$. Recall that $f_{s}$ and $l_{e}$ must satisfy (6.2) and (6.1), respectively.

It was used conservative values for calculating the table parameters. The minimum wavelength $\left(\lambda_{\min }\right)$ considered is $5.075 \mathrm{~mm}$ which is the value at the maximum frequency $\left(f_{\max }=500 \mathrm{kHz}\right)$. The maximum frequency is taken as the frequency where the signal spectrum is surely vanished (see Fig. 6.3 and 6.6.a). The maximum velocity $\left(c_{\max }\right)$ is $3170 \mathrm{~m} / \mathrm{s}$, which is the maximum 
between the maximum phase and group velocity for the A0 mode (see Fig. 5.1 and 5.2 recalling that plate thickness is $d=2 h=3 \mathrm{~mm}$ ).

Table 6.2: FEM A0 results.

\begin{tabular}{cc|cccc|cc}
$l_{e}$ & $f_{s}$ & $\frac{f_{s}}{c_{\max } / l_{e}}$ & $\frac{f_{s}}{f_{\max }}$ & $\frac{d}{l_{e}}$ & $\frac{\lambda_{\min }}{l_{e}}$ & $\begin{array}{r}\text { rmse } \\
(\mathrm{m} / \mathrm{s})\end{array}$ & $\begin{array}{c}\frac{\mathrm{rmse}}{\text { mean }} \\
(\%)\end{array}$ \\
\hline 0.300 & 20 & 1.89 & 40 & 10 & 16.92 & 10.3835 & 0.5266 \\
0.300 & 40 & 3.79 & 80 & 10 & 16.92 & 11.0129 & 0.5585 \\
0.150 & 40 & 1.89 & 80 & 20 & 33.83 & 2.2381 & 0.1135 \\
0.100 & 40 & 1.26 & 80 & 30 & 50.75 & 0.6829 & 0.0346 \\
0.100 & 60 & 1.89 & 120 & 30 & 50.75 & 0.7840 & 0.0398 \\
0.075 & 60 & 1.42 & 120 & 40 & 67.67 & 0.5980 & 0.0303
\end{tabular}

These results reveal that thinner spatial resolutions provide more accurate results. It is then suggested that nodes distance lower than 20 times the minimum wavelength $(N \geq 20$ in (6.1)) is recommended, because when exceeding this value the error significantly diminishes. All the simulations respected condition (6.2) with $f_{s} /\left(c_{\max } / l_{e}\right) \geq 1$. Simply increasing the temporal resolution, without increasing the spatial resolution, when condition (6.2) is already satisfied brings no benefit. This is illustrated in experiments $l_{e}=0.300 \mathrm{~mm}$ at $f_{s}=20 \mathrm{MHz}$ and $f_{s}=40 \mathrm{MHz}$, which present practically equivalent results, actually the highest frequency provided a slightly worse result. The exactly same behavior happened for the experiments $l_{e}=0.100 \mathrm{~mm}$ at $f_{s}=40 \mathrm{MHz}$ and $f_{s}=60 \mathrm{MHz}$.

\subsection{2 \\ S0 mode}

For the S0 mode the excitation signal is a sinc with a $-6 d B$ bandwidth up to $800 \mathrm{kHz}$ in order to encompass the high dispersive region at about $2000 \mathrm{kHz} \times \mathrm{mm}$. This signal is exhibit in Fig. 6.7
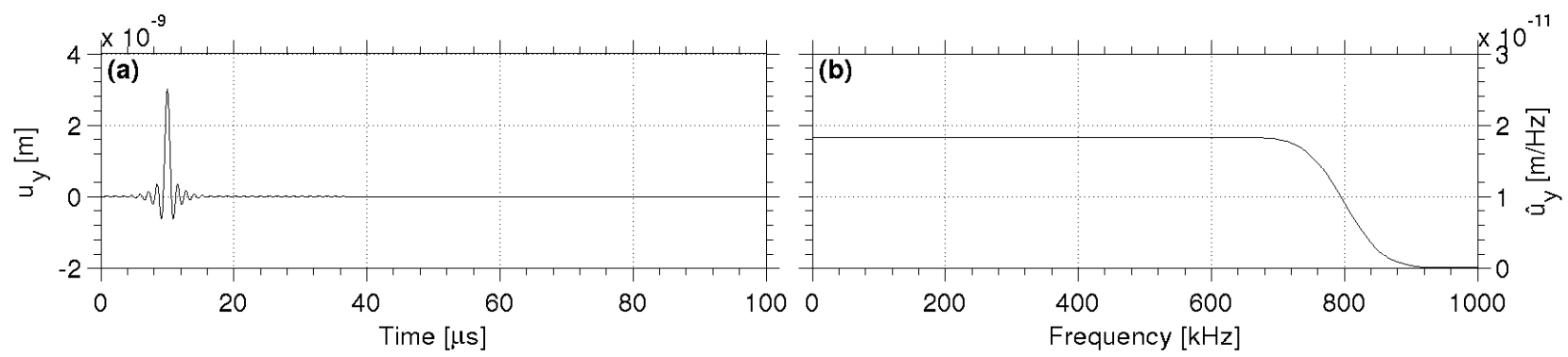

Figure 6.7: S0 excitation signal. (a) Signal in time domain. (b) Signal in frequency domain. 
Fig. 6.8 shows the in-plane displacement response, for a model with $l_{e}=0.100 \mathrm{~mm}$ and sampling frequency of $60 \mathrm{MHz}$. The modeled plate is $450 \mathrm{~mm}$ long. As this mode velocity is higher than the velocity of A0 mode, for the chosen time window $(102.4 \mu s)$, reflections may occur at the plate edge. Fig. 6.8.a and Fig. 6.8.b shows the in-plane $\left(u_{x}\right)$ response, in a grayscale map and seismogram-like plot respectively, for the model without absorbing boundary condition, i.e., where reflections are free the occur. It is possible to observe the reflected wave at the positive plate end propagating toward the origin, at the top right corner of Fig. 6.8.a, and the reflected wave at the negative end, at the bottom right corner of Fig. 6.8.a. The same model with absorbing boundary condition was simulated. Its response is shown in Fig. 6.8.c and Fig. 6.8.d. The absorbing capability introduced by MLK-NRB method is notable as no reflected waves are detectable. This methodology allows one to simulate an infinite medium.

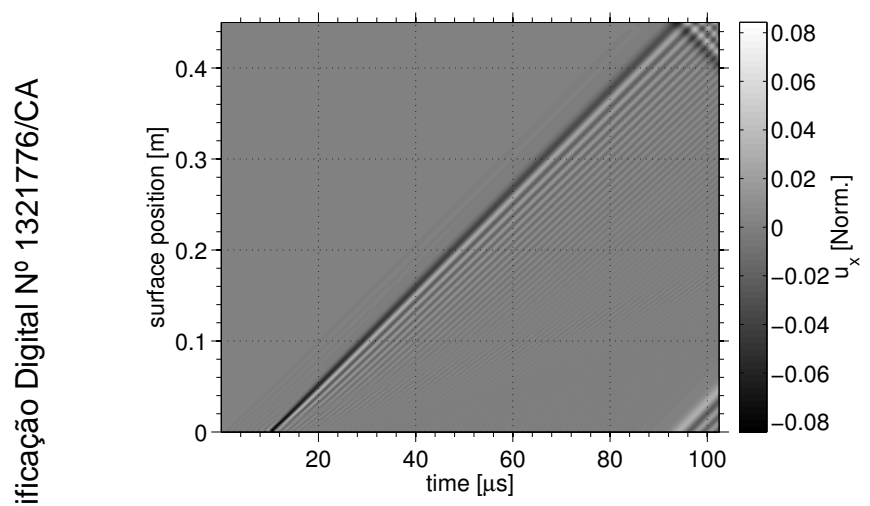

(a)

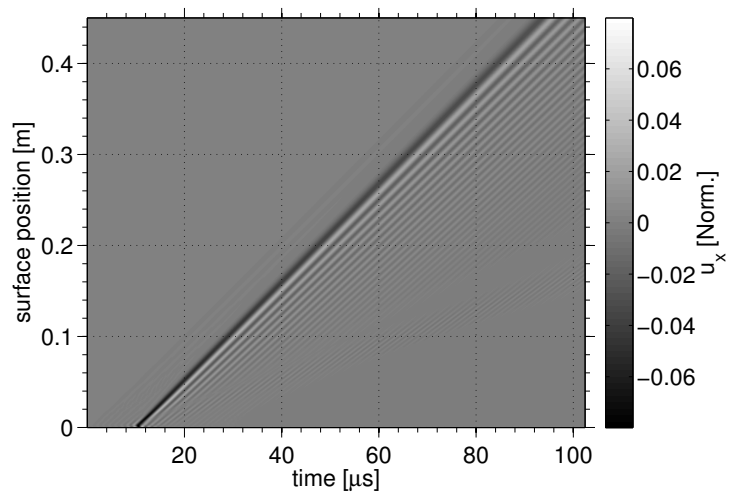

(c)

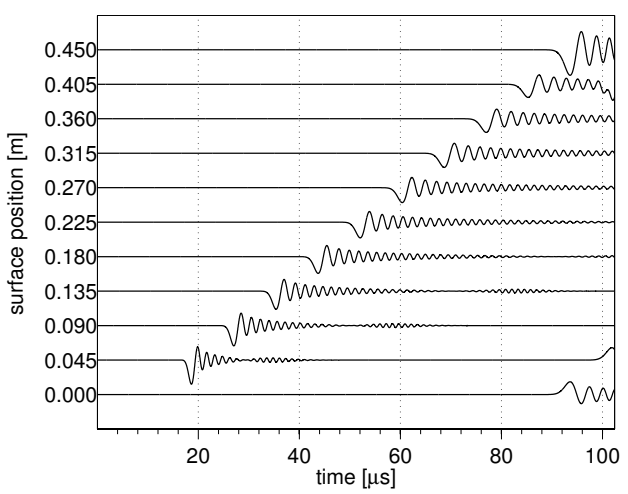

(b)

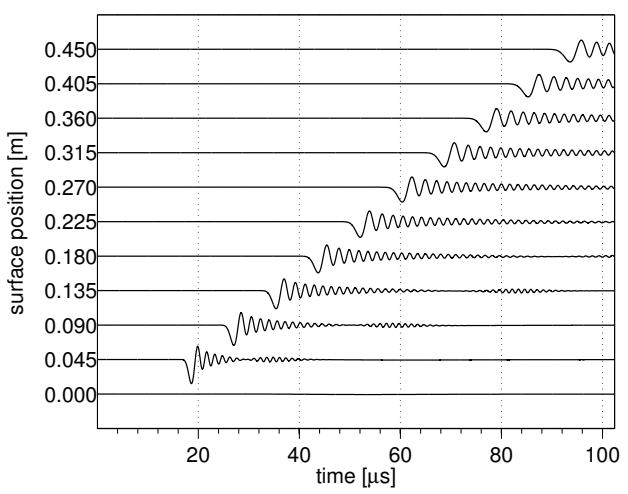

(d)

Figure 6.8: S0 propagation sensed as in-plane displacement at top surface. (a) and (b) without absorbing boundary condition, (c) and (d) With absorbing boundary condition. (a) and (c) Grayscale map. (b) and (d) Seismogram-like plot. 
As it was done for the A0 mode, the two-dimension Fourier transform and phase spectral analysis were performed. Fig. 6.10 shows the two-dimension Fourier transform and the respectively extracted curve.

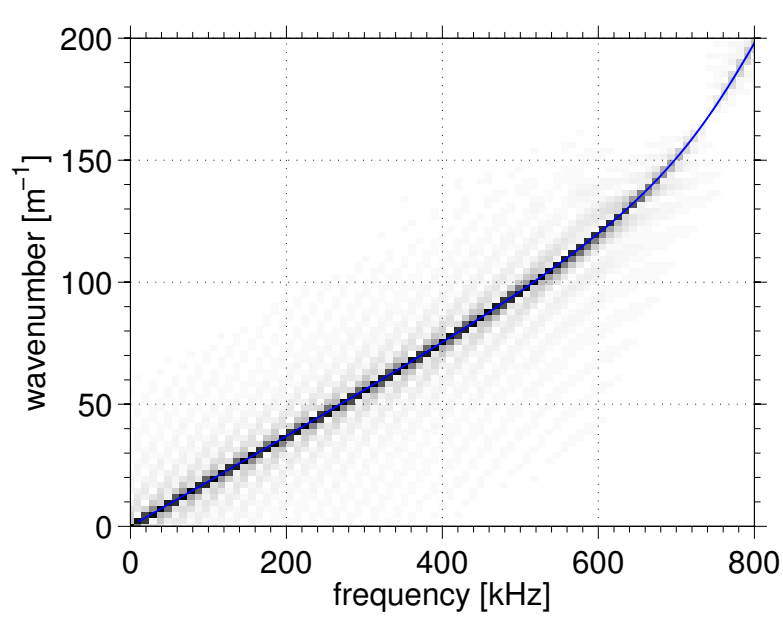

(a)

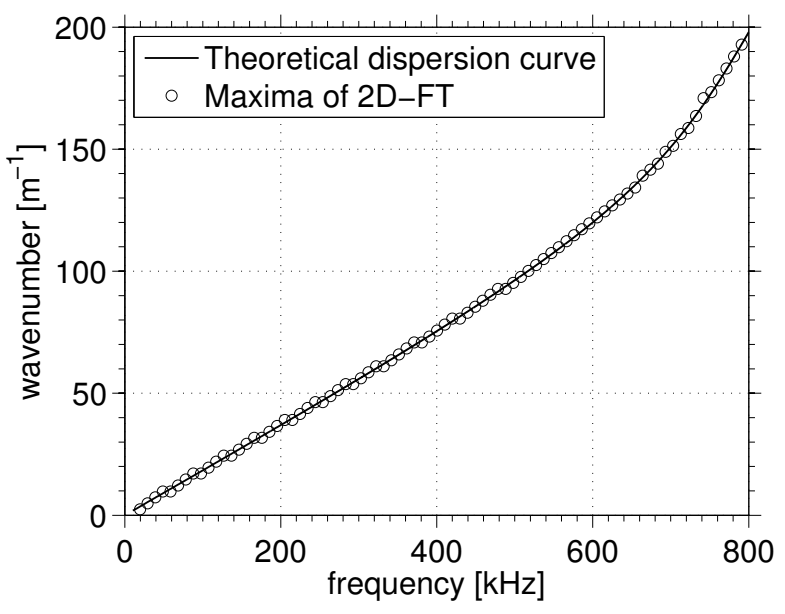

(b)

Figure 6.9: S0 dispersion extraction by two-dimension Fourier transform. (a) Two-dimension Fourier transform absolute value in reverse grayscale map and theoretical dispersion curve represented by the blue line. (b) Extracted dispersion curve.

The phase velocity is directly extracted from the time-trace responses for the S0 mode by the phase spectral method. Fig. 6.10.a shows the in-plane displacement for the positions $x=0.0561 \mathrm{~mm}$ and $x=0.0571 \mathrm{~mm}$ for a time window from 10 to $60 \mu \mathrm{s}$, and their Fourier transform amplitude and phase. The extracted phase velocity for the signal bandwidth is shown in Fig. 6.10.b together with the theoretical value. Again both results coincide, and phase spectral analysis gives better results than the two-dimension Fourier transform.

One can note that the signal shape for the S0 mode is far from the initial sinc pulse used for excitation. This is because this signal is the in-plane $\left(u_{x}\right)$ wave that is generated by the mechanical system, i.e., the plate, in response to the out-of-plane $\left(u_{y}\right)$ excitation. Nevertheless, if one is willing to analyze dispersion effects, this is irrelevant because both waves $\left(u_{x}\right)$ or $\left(u_{y}\right)$ suffer dispersion.

The same procedure to analyze the model accuracy was done for the S0 mode. The results for some resolutions are shown in Table 6.3. The minimum wavelength $\left(\lambda_{\min }\right)$ considered is $3.956 \mathrm{~mm}$ at $900 \mathrm{kHz}$, which is the maximum frequency $\left(f_{\max }\right)$, the maximum velocity $\left(c_{\max }\right)$ is $5442 \mathrm{~m} / \mathrm{s}$. Plate thickness (d) is still $3 \mathrm{~mm}$. 


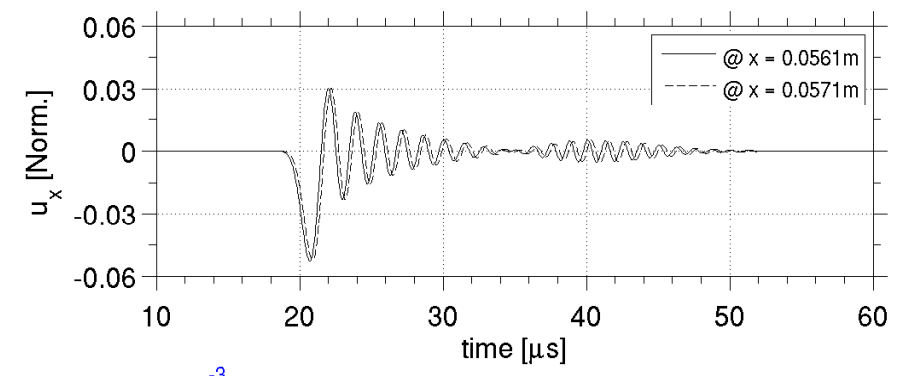

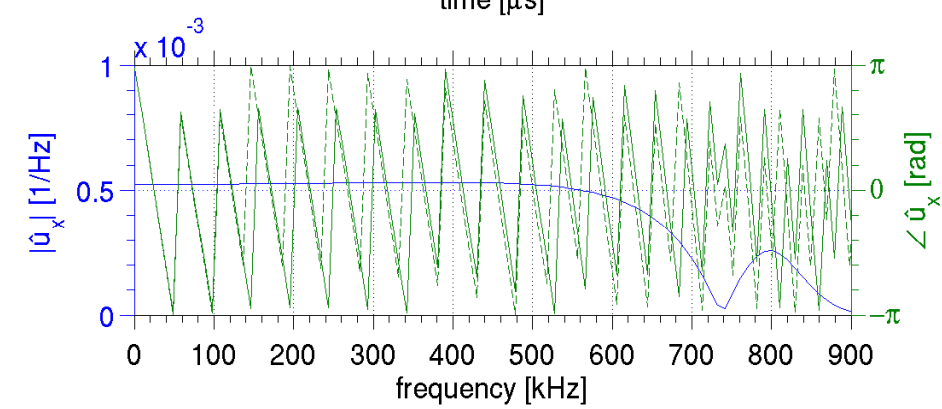

(a)

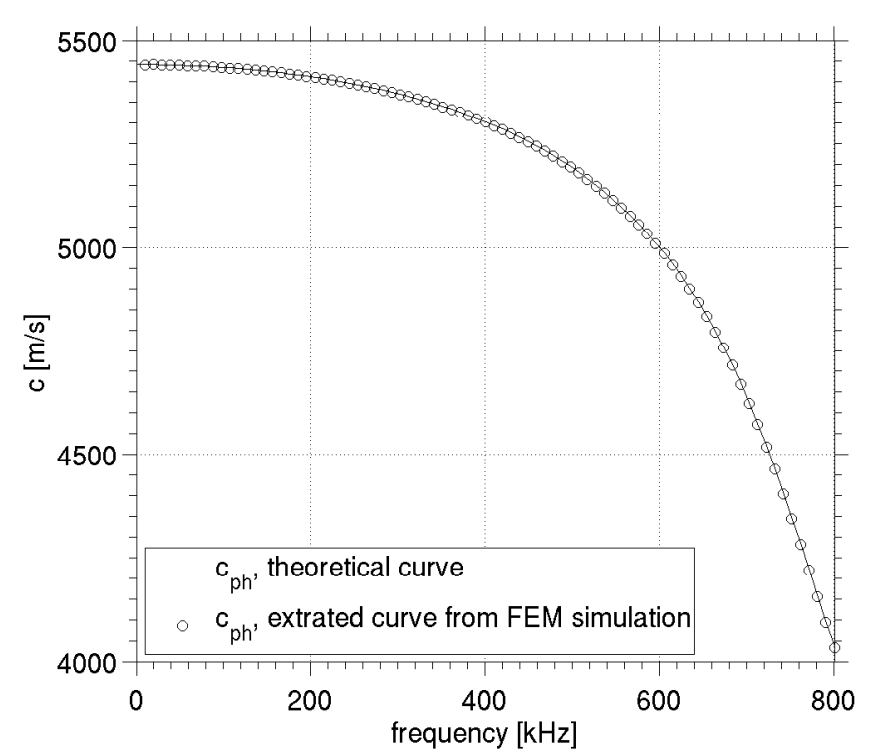

(b)

Figure 6.10: S0 dispersion extraction by phase spectral analysis. (a) Signal at two surface positions, in time domain, top, and their Fourier transform, bottom, in both amplitude, blue lines, and phase, green lines. (b) Extracted phase velocity dispersion curve.

Table 6.3: FEM S0 results.

\begin{tabular}{|c|c|c|c|c|c|c|c|}
\hline $\begin{array}{c}l_{e} \\
(\mathrm{~mm})\end{array}$ & $\begin{array}{c}f_{s} \\
(\mathrm{MHz})\end{array}$ & $\frac{f_{s}}{c_{\max } / l_{e}}$ & $\frac{f_{s}}{f_{\max }}$ & $\frac{d}{l_{e}}$ & $\frac{\lambda_{m i n}}{l_{e}}$ & $\begin{array}{l}\text { rmse } \\
(\mathrm{m} / \mathrm{s})\end{array}$ & $\frac{\text { rmse }}{\text { mean }}$ \\
\hline 0.300 & 20 & 1.10 & 22.22 & 10 & 13.19 & 6.5163 & 0.1239 \\
\hline 0.150 & 40 & 1.10 & 44.44 & 20 & 26.37 & 0.4317 & 0.0082 \\
\hline 0.100 & 40 & 0.74 & 44.44 & 30 & 39.56 & 1.5454 & 0.0293 \\
\hline 0.100 & 60 & 1.10 & 66.67 & 30 & 39.56 & 0.1951 & 0.0037 \\
\hline
\end{tabular}

\section{2}

\section{Simulation for natural reference frame}

In this section the simulation of a loaded plate is performed using the EEC approximation. Note that the accuracy limit for acoustoelastic FEM simulation is the same that was thoroughly examined in section 5.4, and the errors highlight in Figs. 5.19, 5.20 are inherent.

It was performed simulations for both the A0 and S0 mode under $120 \mathrm{MPa}$ of axial longitudinal stress $\left(\sigma_{11}\right)$ in the natural frame of reference, i.e., the stiffness matrix is $\mathbf{A}^{n}$ approximated by EEC. It is here used EEC1 as it was shown to be better suited for this loading case. Thus, for this case the input stiffness matrix is the one presented in (4.43) with the rows and columns 4, 5 and 6 permuted according to the rule set out in the Table 6.1. The excitation is the same as unstressed case, shown in Fig. 6.3 for A0 mode 
and 6.7 for S0.

Fig. 6.11 shows the out-of-plane displacement for A0 mode under unstressed and stressed medium at position $x=140 \mathrm{~mm}$ for a time window from 40 to $100 \mu s$.

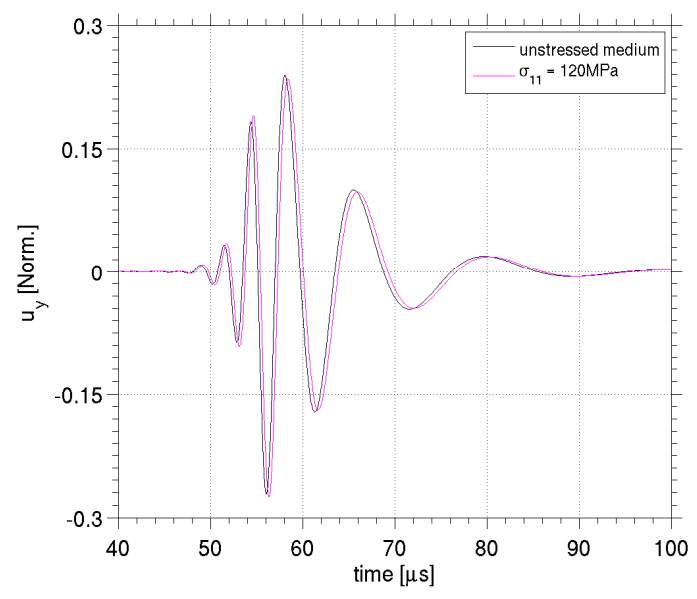

Figure 6.11: A0 FEM response at position $x=140 \mathrm{~mm}$ for a time window from 40 to $100 \mu \mathrm{s}$. Black line is unstressed medium and pink line is for longitudinal axial stress of $120 \mathrm{MPa}$ for EEC1 approximation case.

The dispersion curve is extracted in the same way as it was done for the unstressed medium. Fig. 6.12.a shows the dispersion curves extracted from the simulation for both unstressed and under $\sigma_{11}=120 M P a$ stress, together with the theoretical curves. For the stressed case it is shown both theoretical curves, exactly solution and EEC1 approximation. Observing the wide vision shown in Fig. 6.12.a the curves almost overlay. Figs 6.12.b and c show zoom for low and high frequencies respectively, where it is clear that the simulation for the stressed case match EEC1 approximation and not the exactly solution, as expected. In both zoom regions the curve for stresses medium obtained through FEM are lower than the curve for unstressed medium, as is the behavior of EEC approximation, shown in section 5.4 (Fig. 5.16.b and c). That is, it is not possible to obtain the non-zero velocity for DC components (see Fig. 5.1.b) and the crossing patterns (see Fig. 5.1.e), which are present in the exactly solution, by means of the FEM simulation. As already stated, this is because the FEM simulates an anisotropic medium with stiffness tensor given by EEC1, and thus cannot behave differently from the theoretical curves obtained by this approximation.

The extracted dispersion velocity curve for the S0 mode is shown in Fig. 6.13.a. As the difference from the EEC approximation and exactly solution are more accentuated for higher frequencies (see Fig. 5.20), a zoom at the higher frequencies is shown in Fig. 6.13.b. As for the A0 mode the FEM results match the EEC1 approximated theoretical curve. 


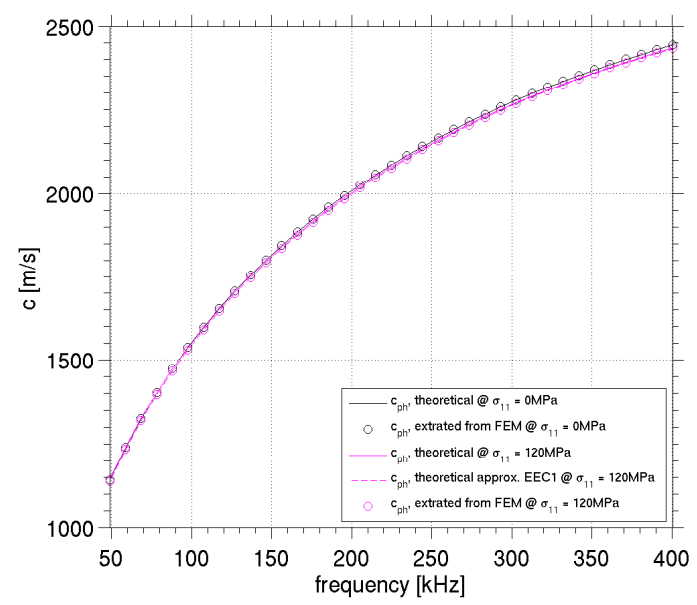

(a)

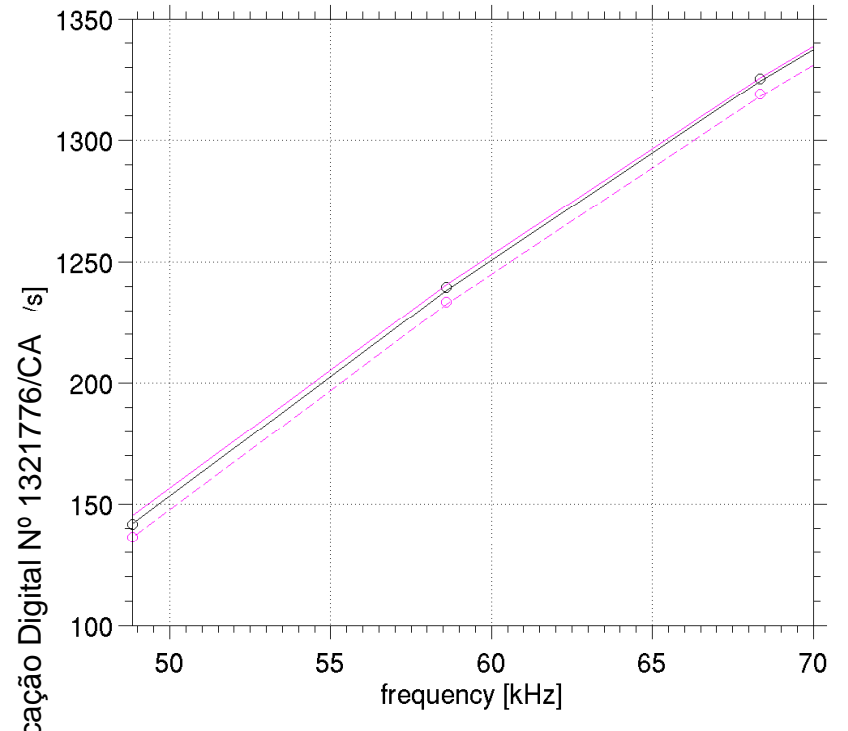

(b)

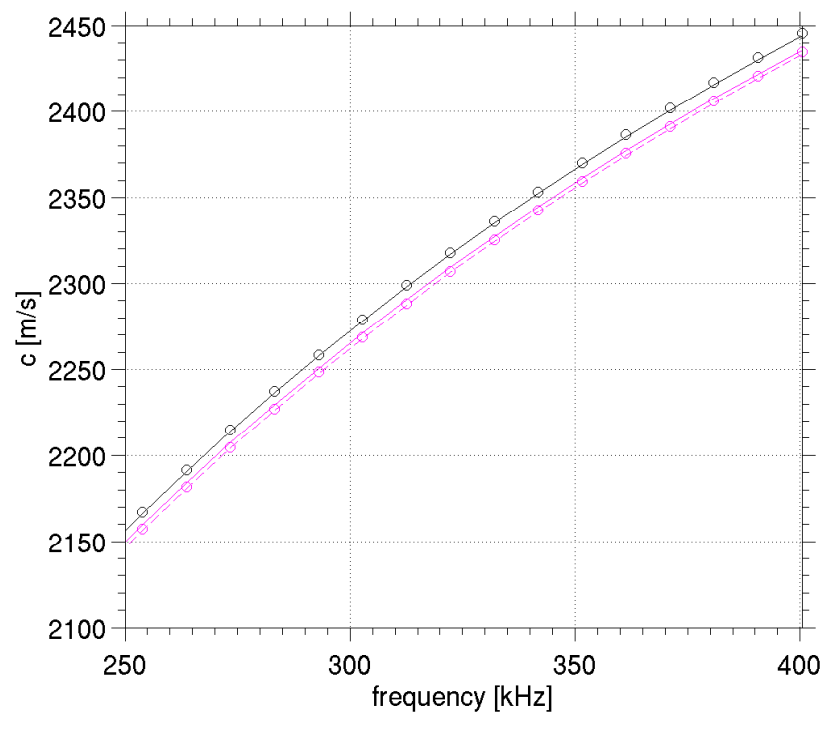

(c)

Figure 6.12: A0 dispersion extraction for stressed medium. Full lines are exactly theoretical curves, dashed lines EEC1 approximation, circles extracted from FEM simulation. Black color stand for unstressed medium, pink for $\sigma_{11}=120 M P a$. (a) Full signal bandwidth and (b) zoom at low frequencies and (c) zoom at high frequencies.

The resolutions that presented the best results for the unstressed medium (Tables 6.2 and 6.3) were tested for accuracy assessment also for the stressed medium. These are presented in Table 6.4 for A0 mode and in Table 6.5 for S0, where the error is related to the theoretical curve obtained for EEC1 approximated. As it can be seen, the error for each resolution are virtually identical than those for unstressed medium. 


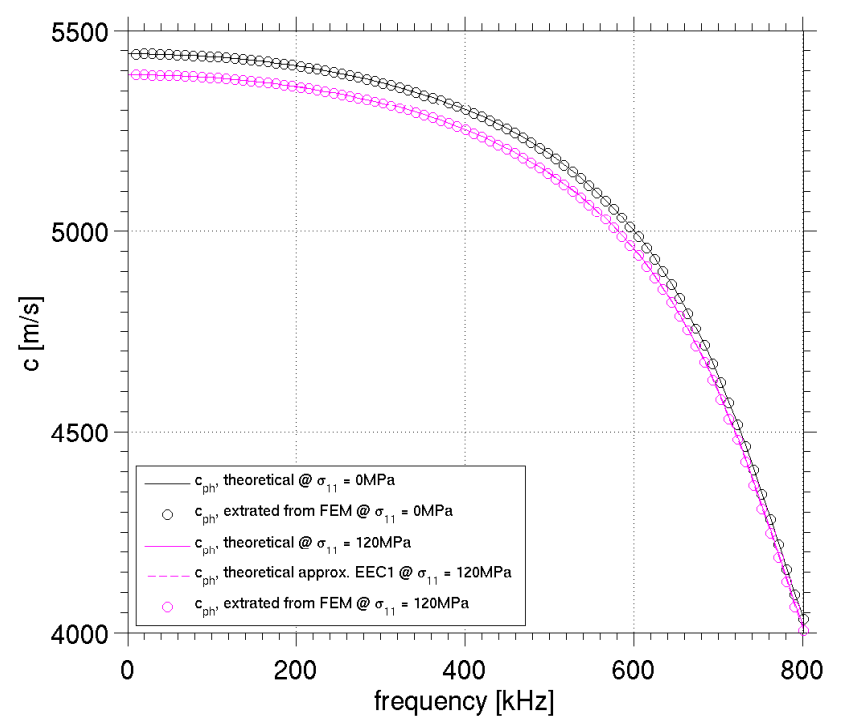

(a)

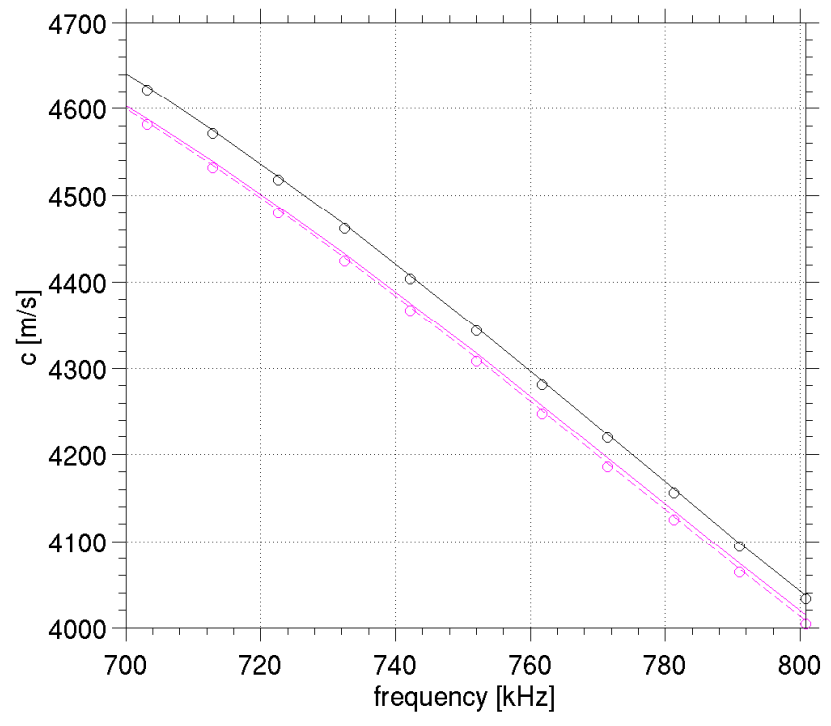

(b)

Figure 6.13: S0 dispersion extraction for stressed medium. Full lines are exactly theoretical curves, dashed lines EEC1 approximation, circles extracted from FEM simulation. Black color stand for unstressed medium, pink for $\sigma_{11}=120 M P a$. (a) Full signal bandwidth and (b) zoom at high frequencies.

Table 6.4: FEM A0 results for stressed medium.

\begin{tabular}{cc|cccc|cc}
$l_{e}$ & $f_{s}$ & $\frac{f_{s}}{c_{\max } / l_{e}}$ & $\frac{f_{s}}{f_{\max }}$ & $\frac{d}{l_{e}}$ & $\frac{\lambda_{\min }}{l_{e}}$ & $\begin{array}{c}\text { rmse } \\
(\mathrm{m} / \mathrm{s})\end{array}$ & $\begin{array}{c}\frac{\mathrm{rmse}}{\text { mean }} \\
(\%)\end{array}$ \\
\hline 0.150 & 40 & 1.89 & 80 & 20 & 33.83 & 2.2370 & 0.1135 \\
0.100 & 40 & 1.26 & 80 & 30 & 50.75 & 0.6813 & 0.0346 \\
0.100 & 60 & 1.89 & 120 & 30 & 50.75 & 0.7831 & 0.0397
\end{tabular}

Table 6.5: FEM S0 results for stressed medium.

\begin{tabular}{|c|c|c|c|c|c|c|c|}
\hline $\begin{array}{c}l_{e} \\
(\mathrm{~mm})\end{array}$ & $\begin{array}{c}f_{s} \\
(\mathrm{MHz})\end{array}$ & $\frac{f_{s}}{c_{\max } / l_{e}}$ & $\frac{f_{s}}{f_{\max }}$ & $\frac{d}{l_{e}}$ & $\frac{\lambda_{\text {min }}}{l_{e}}$ & $\begin{array}{l}\text { rmse } \\
(\mathrm{m} / \mathrm{s})\end{array}$ & $\begin{array}{c}\frac{\text { rmse }}{\text { mean }} \\
(\%)\end{array}$ \\
\hline 0.150 & 40 & 1.10 & 44.44 & 20 & 26.37 & 0.4138 & 0.0078 \\
\hline 0.100 & 40 & 0.74 & 44.44 & 30 & 39.56 & 1.5098 & 0.0287 \\
\hline 0.100 & 60 & 1.10 & 66.67 & 30 & 39.56 & 0.2083 & 0.0040 \\
\hline
\end{tabular}

\section{3}

\section{Simulation for initial reference frame}

One can simulate the effect of load in the initial frame of reference. For that, the tensor $\mathbf{A}^{i}$ from (3.41) must be used as the stiffness matrix using an EEC approximation. Extra attention should be drawn to the model geometry, because when addressing a problem in the initial state, the initially stressed body must be known. This mean that the geometry where the excitation is applied is supposed to represent the initially stressed body. If one does 
not know the deformed geometry, and still wants to address the problem in the initial frame, then the deformed geometry should be initially obtained. This can be done by calculating the initial dimensions, by means of the linear elasticity theory, or even by statical FEM analysis if the geometry is considerably complicated. Then the new (initial) model can be used for the wave propagating analysis. It is important to highlight that all results obtained are thus related to the initial geometry, i.e., if velocity is extracted, then this is the velocity in the initial frame.

The A 0 and S0 mode have been simulated. The model has been redefined due to the initial deformation. This means that the new length is elongated to $L_{i}=L_{0}\left(1+\varepsilon_{11}\right)$ and the new thickness to $d_{i}=d_{0}\left(1+\varepsilon_{22}\right)$, where $L_{0}$ and $d_{0}$ is the natural length and thickness respectively. In the present cases $d_{0}=3 \mathrm{~mm}$.

The same procedure as before was done. The extracted velocity from simulation match the EEC1 theoretical curves. The finest resolutions were examined for the accuracy, related to the theoretical EEC1. The error values are the same, as shown Tables 6.6 and 6.7. This concludes that this procedure allows to simulate the acoustoelastic wave propagation under the EEC approximation.

Table 6.6: FEM A0 results for stressed medium at initial frame of reference.

\begin{tabular}{cc|cccc|cc}
$l_{e}$ & $f_{s}$ & $\frac{f_{s}}{c_{\max } / l_{e}}$ & $\frac{f_{s}}{f_{\max }}$ & $\frac{d}{l_{e}}$ & $\frac{\lambda_{\min }}{l_{e}}$ & $\begin{array}{c}\text { rmse } \\
(\mathrm{m} / \mathrm{s})\end{array}$ & $\begin{array}{c}\frac{\mathrm{rmse}}{\operatorname{mean}} \\
(\%)\end{array}$ \\
\hline 0.100 & 40 & 1.26 & 80 & 30 & 50.75 & 0.6829 & 0.0346 \\
0.100 & 60 & 1.89 & 120 & 30 & 50.75 & 0.7857 & 0.0399
\end{tabular}

Table 6.7: FEM S0 results for stressed medium at initial frame of reference.

\begin{tabular}{|c|c|c|c|c|c|c|c|}
\hline & & $f_{s}$ & $f_{s}$ & $d$ & $\underline{\lambda_{\text {min }}}$ & & rmse \\
\hline $\begin{array}{c}{ }_{e} \\
(\mathrm{~mm})\end{array}$ & $\begin{array}{c}\int_{s} \\
(\mathrm{MHz})\end{array}$ & $\overline{c_{\max } / l_{e}}$ & $\overline{f_{\max }}$ & $\overline{l_{e}}$ & $\overline{l_{e}}$ & $(\mathrm{~m} / \mathrm{s})$ & $\begin{array}{c}\text { mean } \\
(\%)\end{array}$ \\
\hline 0.10 & 60 & 1.10 & 66.67 & 30 & 39.56 & 0.2048 & 0.0039 \\
\hline
\end{tabular}




\section{7}

\section{Time reversal of acoustic waves}

The time reversal principle claims that for each wave emitted from a source (which propagates in the medium, suffering possible reflections, refractions and spreading), there is a set of waves that back propagates in the exactly same path, but in opposite sense. These waves coherently arrive in the original source position, as if time was running backwards.

The time reversal of acoustic waves was originally proposed by Mathias Fink at the end of the eighties as a method to obtain focused acoustic waves [48]. In the case of guided Lamb waves, the use of time reversed signals compensates the dispersion of each propagation mode, recompressing the signal and producing a focused signal at the reception point as showed by Ing et al. [51], which was one of the first applications of the time reversal technique on Lamb waves. In that work, Lamb modes have been excited in a thin plate by a short pulse with large frequency spectrum. An array of elements was used as sensor for time reversing and retransmitting the set of signals. The initial short pulse was successfully re-compressed, showing the time focusing capability. It was also demonstrated that the finite plate width combine both guidance principles, by the width and thickness, and so enhances the focusing quality.

The time reversal technique applied to Lamb waves found a promising field in NDE area. When the time reversal is applied to Lamb waves excited by a narrow-band tone burst, the original signal is completely regenerated for a defect free plate, but the received signal differs from the original in the presence of a defect. Therefore, it provides a baseline-free procedure in NDE, where no information of an undamaged specimen is needed [140-144]. In Jeong et al. [145] it was shown that the multi-path caused by reflections at a defect generates side lobes at the time reversal focused signal. Positioning the defect was possible by correlating the lobe position with the phase and group velocities.

It is worth to point out that, due to the transfer function amplitude dependence on frequency for the Lamb waves, it is necessary to use a narrow band excitation if one is interested in recovering the original signal. Wang et al. [140] and Park et al. [49] analytically investigated this dependency by means 
of the Mindlin plate theory [146].

Wang et al. [140] used the time reversal principle in a sensor network for a continuous health monitoring system. Park et al. [142] also used the time reversal in plates, with two transducer and detailed described the process in the case of a narrow band excitation [49]. In Poddar et al. [144] a thorough selection for better frequency and mode values were performed aiming to enhance signal reconstruction; the effects on the time reversal signal for different types of defect was also observed. In Kerbrat et al. [147] it is used a phased array transducer with a variant of the time reversal technique in order to detect and focus in multiple defects in a plate. More recently, Xu et al. [82] proposed a wideband dispersion reversal technique in order to separate the dispersion effect of each mode.

\section{1}

\section{Time reversal in plates}

In this section, the time reversal process in transmission-reception mode is described. The emphasis is placed on the frequency domain.

Both emitter and receiver are fixed transducers, called $T_{e}$ and $T_{r}$, respectively. The transfer function that relates the signal emitted by $T_{e}$ and received by $T_{r}$ is named $H_{e r}$. Due to the reversibility of the piezoelectric effect and the reciprocity in the wave propagation [148], the transfer function $H_{e r}$ is assumed to be equal to $H_{r e}$ and henceforth named $H$.

For better understanding, the time reversal process can be split into four steps. In the first step, a short impulse signal $x_{0}(t)$ is emitted by $T_{e}$. The acoustic field produced by this first pulse propagates according to the medium characteristics. Such as, attenuation, phase and group velocity, dispersion, possible modes distribution and reflections.

Next, at step 2, the received signal at the opposite transducer $T_{r}$, say $y_{0}(t)$, is recorded. It is defined a practical acquisition window that starts at the instant $t_{0}$ and ends at $t_{0}+\Delta T$. Steps 1 and 2 are illustrated in Fig. 7.1.

At step 3, the last signal is time-reversed and retransmitted from $T_{e}$ to $T_{r}$. Note that it is exactly the same as transmitting from $T_{r}$ to $T_{e}$, due to reciprocity. The reversed signal can be expressed as

$$
\begin{aligned}
& x_{1}(t)=y_{0}\left(t_{0}+\Delta T-t\right), t_{0} \leq t \leq t_{0}+\Delta T, \quad \text { or simply, } \\
& x_{1}(t)=y_{0}(-t), \quad \text { where the shift delay is omitted. }
\end{aligned}
$$

Finally, at step 4 , the signal $y_{1}(t)$ is received at the transducer $T_{r}$ and recorded. Steps 3 and 4 are illustrated in Fig. 7.2. 

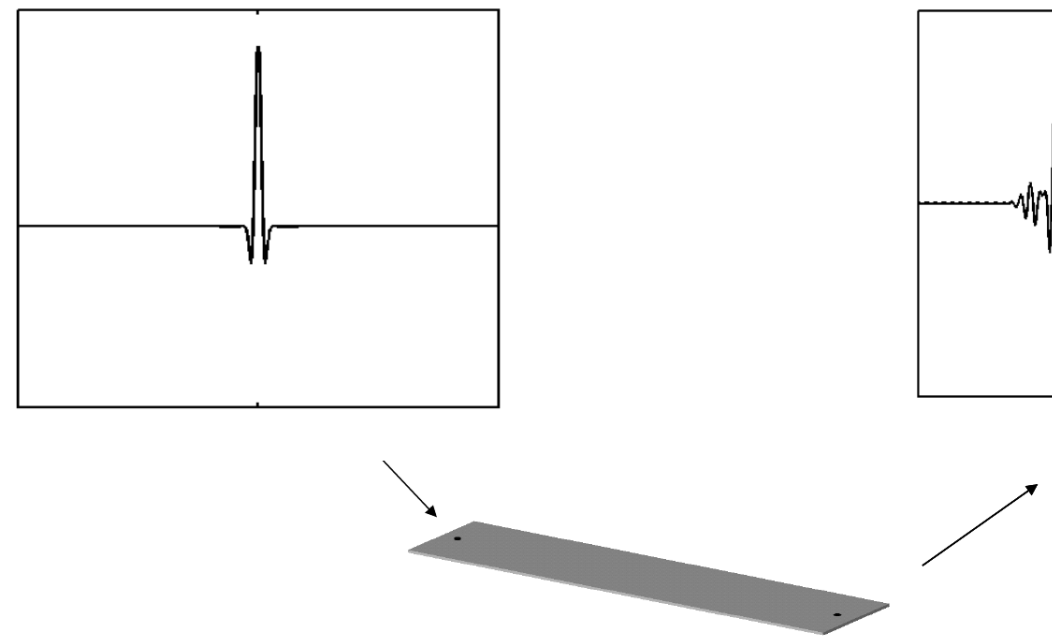

Figure 7.1: Time reversal process, steps 1 and 2. At the left, a sharp pulse is used for excitation (step 1). At the right, the forward propagated signal is received (step 2) due to medium propagation characteristic as dispersion and multi-modal behavior.

The signal received at step 2, i.e., the forward propagation signal, can be represented by the series link between the electric-acousto response of transmitting transducer $\left(a_{t x}\right)$, acoustic system response $(g)$, and acoustoelectric response of receiving transducer $\left(a_{r x}\right)$, i.e.,

$$
\begin{aligned}
y_{0}(t) & =x_{0}(t) * a_{t x}(t) * g(t) * a_{r x}(t), \text { in time domain, or } \\
\hat{y}_{0}(f) & =\hat{x}_{0}(f) \cdot \hat{a}_{t x}(f) \cdot \hat{g}(f) \cdot \hat{a}_{r x}(f), \text { in frequency domain, }
\end{aligned}
$$

where $*$ is the convolution operation. The last three terms in (7.4) is the system transfer function. In the frequency domain this is given by

$$
\widehat{H}(f)=\hat{a}_{t x}(f) \cdot \hat{g}(f) \cdot \hat{a}_{r x}(f)
$$

where it is clear that the transfer function depends on the acousto-electric responses of both transducers and the physics of wave propagation, represented by $\hat{g}(f)$. In the plate, this is formed by the contribution of the Lamb wave modes.

Thus, the received signal at the second step is written in the frequency domain as

$$
\widehat{y}_{0}(f)=\widehat{x}_{0}(f) \widehat{H}(f) \text {. }
$$

The negative sign in time domain, $-t$, is equivalent to the conjugate signal of its Fourier transform in the frequency domain. This is the timereversal property of the Fourier transform [76]. So, at the third step, the spectrum of the retransmitted signal is the complex conjugate of the received signal, times a global phase factor that arises because of the time shift. In the 

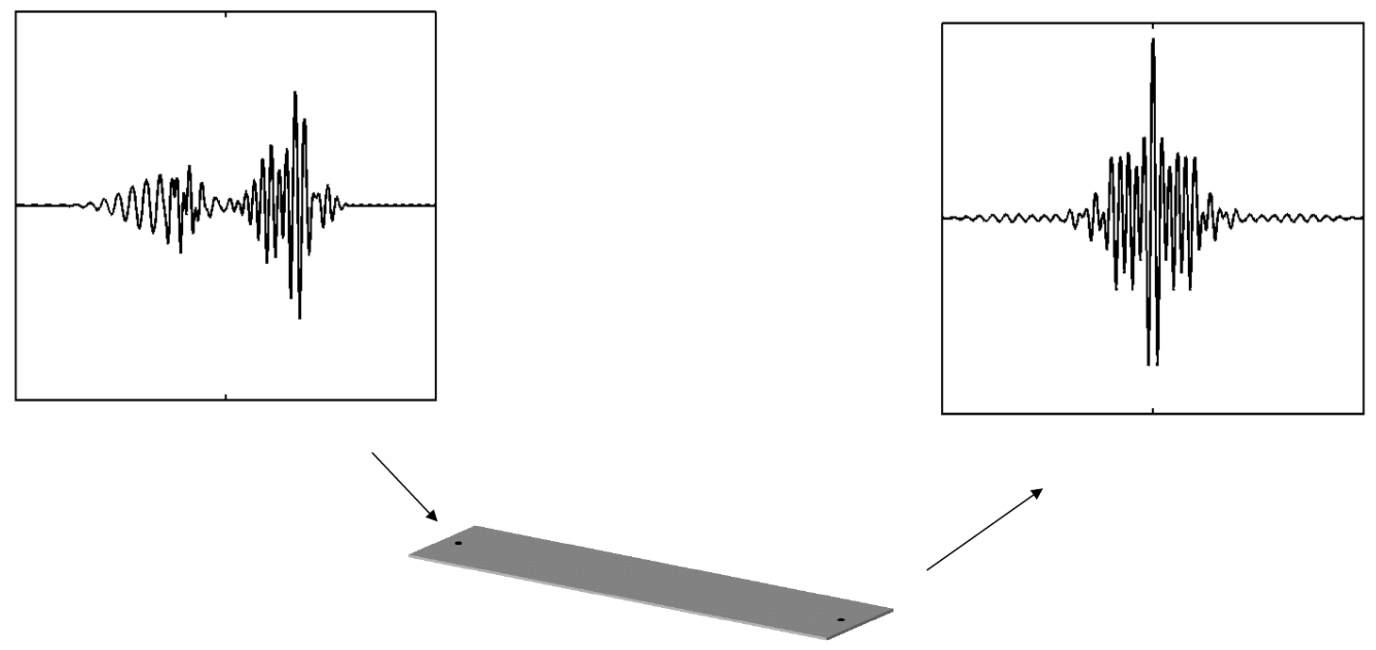

Figure 7.2: Time reversal process, steps 3 and 4 . At the left the time-reversed signal is used as excitation (step 3). At the right the focused signal is received (step 4).

same way as done for the time-domain expression, the global phase factor for the frequency-domain will be omitted. Considering this, (7.2) is written in the frequency domain as

$$
\widehat{x}_{1}(f)=\widehat{y}_{0}^{*}(f) \quad .
$$

The transmitted signal at step 3 passes again by the system transfer function as it propagates. Considering it, the received signal at step four is

$$
\widehat{y}_{1}(f)=\widehat{x}_{1}(f) \widehat{H}(f),
$$

and applying (7.6) and (7.7) into (7.8) results in the following expression

$$
\widehat{y}_{1}(f)=\widehat{x}_{0}^{*}(f) \widehat{H}^{*}(f) \widehat{H}(f) .
$$

One can observe that the time-reversal process can be computed in the frequency domain as the product of the transfer function, $\widehat{H}(f)$, by its complex conjugate, which is equal to the square of transfer function amplitude.

Analytically, the signal can be returned to the time domain by using inverse Fourier transform as,

$$
y_{1}(t)=\int_{-\infty}^{\infty} \widehat{x}_{0}^{*}(f)|\widehat{H}(f)|^{2} e^{i 2 \pi f t} d f
$$

In order to ensure flat frequency content inside a predefined bandwidth, one can use a sinc pulse as $x_{0}(t)$. In this case $\widehat{y}_{0}(f)$, in step 2 , is proportional to $\widehat{H}(f)$, inside the excitation bandwidth (see (7.6)). Considering this particular case, the spectrum of the initial pulse is assumed constant, say equal to $X_{0}$, if $f$ belongs to the bandwidth $B W$ and is zero otherwise, and then (7.10) simplifies to 


$$
y_{1}(t)=X_{0} \int_{B W}|\widehat{H}(f)|^{2} e^{i 2 \pi f t} d f
$$

It can be noticed that for the instant $t=0$ all frequency components have the same null phase. This implies that all frequency spectra are placed in phase at this instant, resulting in a focused signal. This justifies how the time reversal process can compensate the intrinsically dispersive behavior of the guided waves, as illustrated in Fig.7.2. At this time, the amplitude of the focused signal is maximum and proportional to the energy of the system, as can be directly observed from (7.11). Other property that can be observed directly from the equations is the symmetry related to the peak instant.

Actually, the focusing time is not really zero, it is equal to the final instant of the temporal acquisition window of received signal at step 2, i.e., $t 0+\Delta T$, which has been omitted in the above expressions.

The time reversal process can also be interpreted from the propagation point of view. When one uses the time-reversed signal as excitation, in step 3, the exact reversal dispersion and relative delay for each mode is imposed in order to compensate the dispersion and the modes separation at the receiver position resulting in a coherent sum at the proper time, composing the signal received in step 4 . In other words, all modes and all mode frequency components arrive coherently at the receiver position.

Expressions (7.9) and (7.11) are valid only for linear time invariant systems. However, external factors, such as temperature or mechanical strain, can introduce changes in the frequency response. In the forthcoming section 7.4, the effect of initial strain on the time reversal process is theoretically analyzed.

\section{2}

\section{Time reversal energy efficiency}

As can be expected from expression (7.11), the time reversal focusing technique concentrates more energy the wider is the original signal spectrum. One might use the spectrum bandwidth for comparing the spectrum content. However, in a practical case, the spectrum shape is far from regular; it may contain many peaks and valleys (some real experimental transfer functions are presented Chapter 9). Thus, a simple bandwidth comparison is not obvious because it may consider regions of no spectrum relevance, or even neglect some frequency parts that may yet present some importance. To quantify the timereversal temporal focusing quality, the energy focusing efficiency is proposed here. Time reversal energy focusing efficiency (TREF) is defined as the ratio of the energy in the central peak divided by the global signal energy. The 
central peak is here defined as the signal part between the two zeros crossing immediately before and after the main positive lobe, as illustrated by the filled areas in Fig. 7.3, where an artificial signal is shown. Its formal definition is stated in (7.12),

$$
T R E F\left\{y_{t r}(t)\right\}=\frac{E_{p k}}{E_{t o t}},
$$

where $E_{t o t}$ is the total signal energy and $E_{p k}$ is the energy in the focused interval defined, respectively, as

$$
\begin{aligned}
& E_{t o t}=\int_{-\infty}^{\infty} y_{t r}^{2}(t) d t \\
& E_{p k}=\int_{t_{1}}^{t_{2}} y_{t r}^{2}(t) d t
\end{aligned}
$$

where $t_{1}$ and $t_{2}$ are the two zeros crossing immediately before and after the main positive lobe; which define the peak interval.

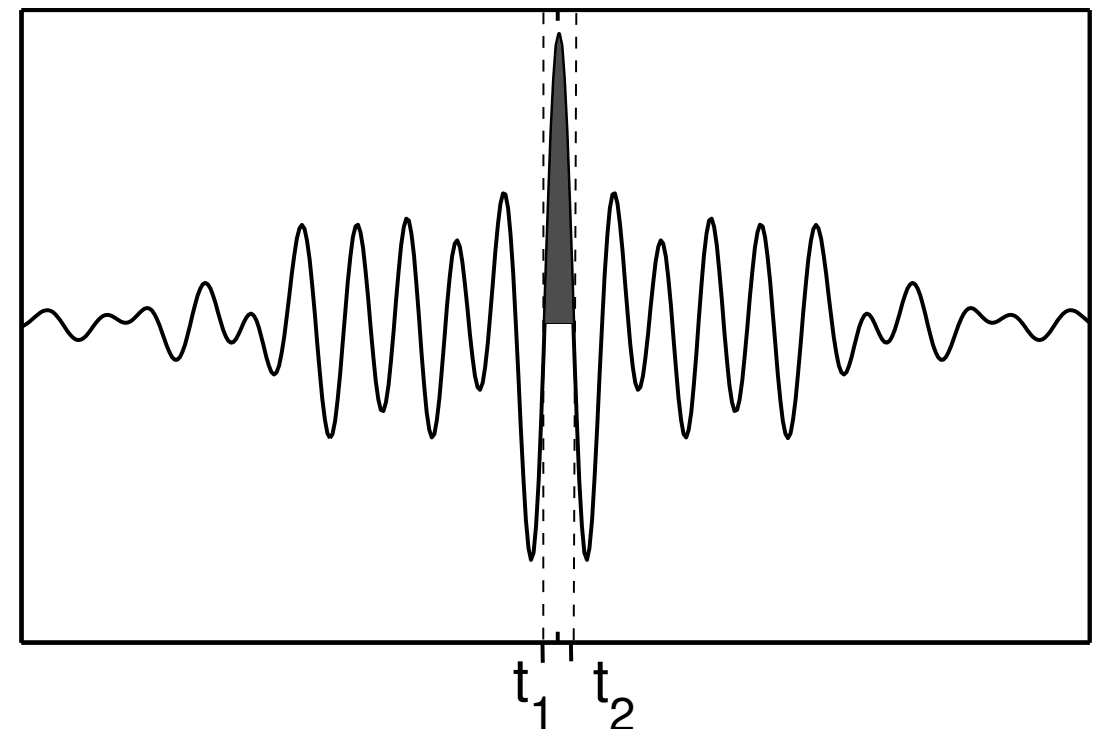

Figure 7.3: Example signal for time reversal energy efficiency. The instants $t_{1}$ and $t_{2}$ and the area used for energy calculating are shown.

A Dirac delta signal would represent a perfect time reversal and its ratio would be unitary, as the whole energy is confined in the central peak. On the other hand, for a totally unfocused signal, such as a sine wave where the bandwidth is null, no energy is focused and the ratio approaches zero. This definition is close to the signal to noise ratio. However, in this case, the energy out of the focus is not really noise, but rather a deterministic signal produced by the time reversal process. This measure represents a way of indirectly measuring the spectral equalization level, or even its richness, without either analyzing its frequency content or concerning its possible irregularities. 


\section{3}

\section{Time reversal spatial focusing}

The spatial focus of the time reversal process represent the specificity of focusing to the position. In other words, it represents how intense is the wave field around the proper focus position. It is normally measured as the peak, or the peak-to-peak, value versus the distance from the focus position, in some axis of interest. In order to measure it one has to sweep the sensing transducer around the focus position. It typically exhibits a pattern like the one shown in Fig. 7.4.

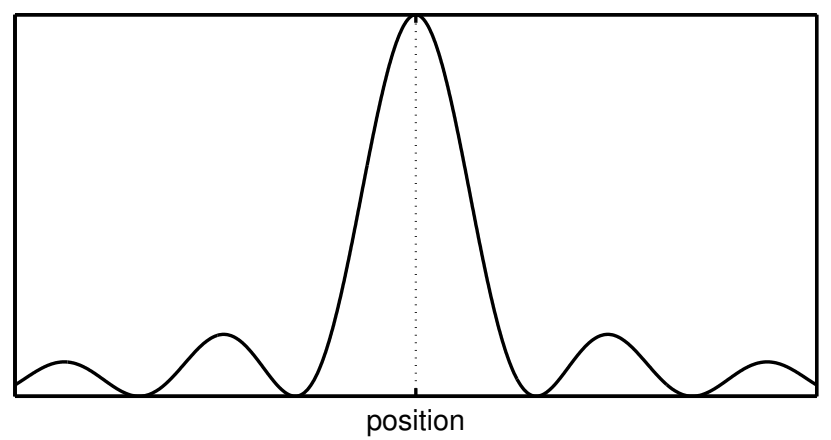

Figure 7.4: Example of typical spatial focus pattern. Abscissa is the distance from the focus position and ordinate is the peak value.

The thinner is the main focus lobe in the spatial focus, the more specific is the spatial focus (or the better the focus). It was experimentally demonstrated that for Lamb waves the more dispersive is the working setup, the higher is the spatial focusing quality [51].

\section{4}

\section{Effect of longitudinal strain on time reversal signal}

The theory exposed in the previous section assumes that the system is time-invariant. This means that the transfer function $H$, identified in the first step, is the same in the next instants, when the signal is retransmitted. In this section the explicit dependence of the time reversal signal on the strain level is introduced. Without external stress, the transfer function is designated as $\widehat{H}(0, f)$ whereas the transfer function at strain level $\varepsilon$ is $\widehat{H}(\varepsilon, f)$.

The focusing ability of the time-reversal process is based on the fact that the transfer function is the same in both halves of the process. Thus, the phase is nullified at the focusing time and the signal is maximized. If the transfer function is altered during steps 3 and 4, related to steps 1 and 2, then there is no more guarantee of the matching of the retransmitted signal with the original transfer function, which implies loss of focusing. This is illustrated in 
Fig. 7.5, in which a different stress condition for steps 3 and 4 is represented by the red arrows.

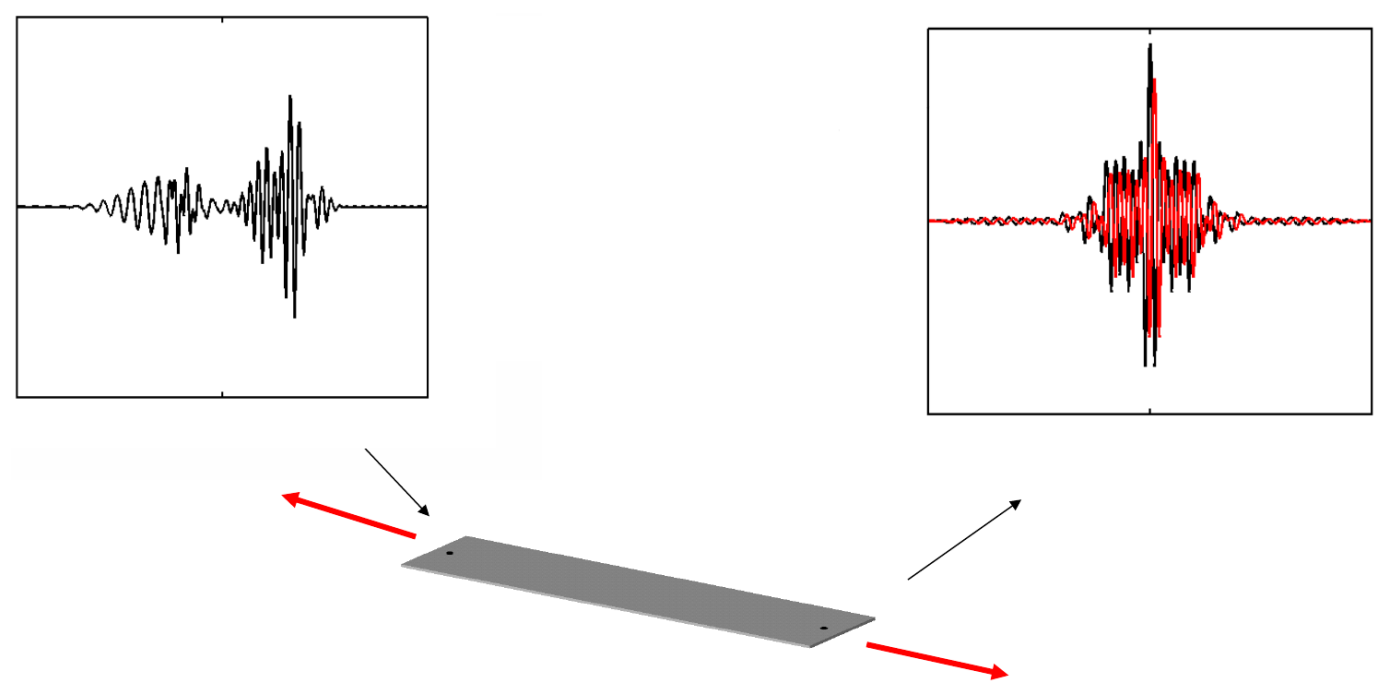

Figure 7.5: Time reversal process under longitudinal, steps 3 and 4. At the left the time-reversed signal is used as excitation referenced to the null strain condition (step 3). The plate is now strained, represented by the red longitudinal arrows. At the right the focused signal is received (step 4), the red signal represents the lose of focusing quality compared to the black signal, received for the same strain conditions as the reference.

The strain effect on the time reversal signal is explicit if the expression of the received signal at step 4, given by (7.9), is modified for accounting for this assumption, as follows

$$
\begin{aligned}
& \widehat{y}_{1}(\varepsilon, f)=X_{0} \widehat{H}^{*}(0, f) \widehat{H}(\varepsilon, f), \quad \text { or, } \\
& \widehat{y}_{1}(\varepsilon, f)=X_{0}|\widehat{H}(0, f)||\widehat{H}(\varepsilon, f)| e^{i[\phi(\varepsilon, f)-\phi(0, f)]},
\end{aligned}
$$

where $\phi(\varepsilon, f)$ is the phase of the transfer function at the $\varepsilon$ strain level. The phase is not canceled independently of the frequency and a phase error is present at the received time reversal signal, degrading the focus.

How the strain change the transfer function is understood by analyzing the changes of propagation condition. The main effect of the tensile load over the signal is a phase shift that depends on the frequency and on the propagation mode. The phase difference occurs due to the dimension variations and velocity changes induced by the stress state, as explains the acoustoelastic theory exposed in Chapter 4.

The transfer function can be expressed by the sum of the Lamb modes as

$$
\widehat{H}(\varepsilon, f)=\sum_{j} m_{j}(f) e^{-i \kappa_{j}(\varepsilon, f) \ell}=\sum_{j} m_{j}(f) e^{-\frac{i 2 \pi f \ell}{c_{j}(\varepsilon, f)}},
$$


where $m_{j}(f)$ is the amplitude of mode $j$ as a function of the frequency, $\kappa_{j}(\varepsilon, f)$ is the wavenumber of mode $j$ at the strain $\varepsilon$, and $c_{j}(\varepsilon, f)$ its phase velocity and $\ell$ the natural propagation distance. Here the acousto-electrical responses are neglected because it is assumed that they are not strain dependent, and so their phases are compensate by the time reversal process. The distance dependence on strain is not included because the velocity is considered at the natural frame of reference, and thus one should use the natural coordinates.

The precise value of the transfer function modulus, $H(\varepsilon, f)$, and phase, $\phi(\varepsilon, f)$, is to be found by evaluating the expression (7.15). The phase dependence on strain is known (derived in Chapter 5). The amplitude distribution of each mode is not a priori defined. If there is just one mode, then the transfer function modulus is the mode amplitude and the phase coincides with the mode phase.

One can introduce a further simplification on the (7.14b) by assuming that strain would introduce meaningful changes in the spectrum phase, holding constant the amplitude of each component. That is, the phase difference is predominant over the changes in the amplitude of the transfer function. By introducing this additional hypothesis, expression (7.14b) is modified as follows.

$$
\widehat{y}_{1}(\varepsilon, f)=X_{0}|\widehat{H}(0, f)|^{2} e^{i \Delta \phi(\varepsilon, f)},
$$

where $\Delta \phi(\varepsilon, f)=\phi(\varepsilon, f)-\phi(0, f)$, is the phase difference of the transfer function between the initial zero strain state and the deformed state at strain level $\varepsilon$. Expression (7.16) can be transformed into the time domain as in (7.11).

$$
y_{1}(\varepsilon, t)=X_{0} \int_{B W}|\widehat{H}(0, f)|^{2} e^{i \Delta \phi(\varepsilon, f)} e^{i 2 \pi f t} d f
$$

As the phase difference is not constant over the frequency range, expression (7.17) explains why there exists a significant peak amplitude decrease, in addition to a global time-shift, in the focused signal, which is achieved by the broadband time reversal break due to strain. Fig.7.6 shows the ideal behavior of the focus changes due to the applied traction.

As stated in section 7.2, the wider is the signal band, more modes are able to participate in the time reversal process. Also the wider is the signal band, more modes can introduce a mismatch on the focusing. Thus, it could be hypothesized that the strain sensitivity shares the same condition of the focusing quality. That is, the higher the TREF, the higher the focusing quality, and also the sensitivity to strain. This relationship is investigated in the next two chapters. 


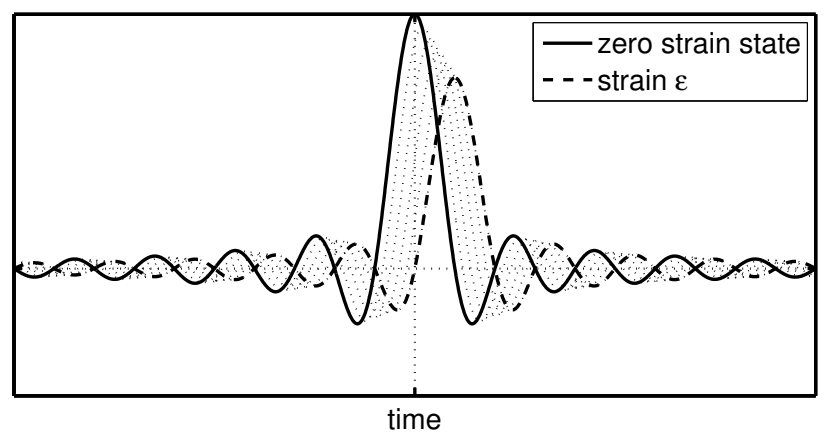

Figure 7.6: Ideal variations of the time reversal focus when an external strain $\varepsilon$ is applied. Observe the decrease of the maximum and the shift in the focalization time

\section{5}

\section{One-bit time reversal in plates}

The one-bit variation of time reversal sets the amplitude of the exciting signal to \pm 1 depending on the sign of the signal received. This version of the time reversal is technically easier to implement since just a voltage switch is required instead of a full analog synthesizer used in the normal technique [149-151].

This variation just uses the sign of signal $x_{1}(t)$, at step 3 , to be inverted and transmitted back [149]. This means that the signal to be retransmitted presents the values 1 or -1 only, i.e., no amplitude information is considered, and just instantaneous phase is transmitted. Actually, the admissible values are the excitation voltage used in the equipment, say $\pm V$. So, step three becomes

$$
x_{1 b i t}(t)=V \operatorname{sign}\left\{y_{0}(-t)\right\} \text {, }
$$

and thus the signal received at step four is

$$
y_{1 b i t}(t)=V \operatorname{sign}\left\{y_{0}(-t)\right\} * y_{0}(t)=\int V y_{0}(\tau) \operatorname{sign}\left\{y_{0}(t+\tau)\right\} d \tau .
$$

The major advantage, besides simplicity, is that the focus gain is maximized [150]. This characteristic can be concluded by analyzing the response to an arbitrary signal, say $x(t)$, and

$$
y(t)=x(t) * y_{0}(t)=\int x(\tau) y_{0}(t-\tau) d \tau,
$$

at the time of focus, becomes

$$
y(0)=\int x(\tau) y_{0}(-\tau) d \tau \leq \int|x(\tau)|\left|y_{0}(-\tau)\right| d \tau .
$$

Applying the constraint that the absolute maximum value of $x(t)$ be less than or equal to $\mathrm{V}$, 


$$
y(0) \leq \int V\left|y_{0}(-\tau)\right| d \tau=\int V \operatorname{sign}\left\{y_{0}(-\tau)\right\} y_{0}(-\tau) d \tau \quad,
$$

and the last equality is the response to the one-bit time-reversed excitation as in (7.18), concluding that the excitation signal that produces the maximum amplitude at the focus instant is the one-bit time-reversal. The above development concerns the amplitude at the focus $(t=0)$ and no other assumption regarding the overall signal shape, as its symmetry or, level outside the focus, or TREF, can be concluded from it. These parameters are dependent on the system. No relationship in the frequency domain is straightforwardly obtained as in (7.9).

Considering that the effect of strain is mainly present in the signal phase, then it affects both the conventional and one-bit versions of the method. In the one-bit implementation, the sign of the reference null strain signal is transmitted; the received signal at the last step for the strained medium is expressed by

$$
y_{1 b i t}(\varepsilon, t)=V \operatorname{sign}\left\{y_{0}(0,-t)\right\} * y_{0}(\varepsilon, t)=\int V y_{0}(\varepsilon, \tau) \operatorname{sign}\left\{y_{0}(0, t+\tau)\right\} d \tau
$$

In this case, an expression that relates the dependence on the time-reversal signal with the relative phase differences, introduced by the strain, is not available, as in (7.14b). However, the focusing properties were shown to be quite similar to those of the traditional time-reversal [149]. 


\section{8 \\ Simulation of wideband time reversal of Lamb waves under longitudinal stress}

The results in chapter 6 validate the simulation framework for the sequence here exposed. In this chapter the propagation of wideband multimodal Lamb waves is investigated through finite element analysis. Then the response at a predefined position is used for the time reversal process. The time reversal signal is obtained and its focusing characteristics are observed for different strain levels. The correlation implementation is confirmed to be equivalent to the physical phenomenon and so it will be treated from then on. The acousto-electrical effect of the transducer is simulated by applying band limited filters to the received signal. Two main situations are used: finite length plate, were reflections do happen, and infinite length plate, simulated by including the absorbers, as introduced in chapter 6 .

The modeled geometry is a $L=800 \mathrm{~mm}$ long, $d=3 \mathrm{~mm}$ thick plate shown in Fig. 6.1. The absorbers are included if an infinite length plate is to be simulated. The wideband exciting signal is a sinc signal with $-6 \mathrm{~dB}$ bandwidth up to $2.5 \mathrm{MHz}$ that is applied as out-of-plane displacement at top surface and origin of $x$ coordinate, i.e., at $x=0, y=+h$. This signal is shown in Fig. 8.1 in both time and frequency domains.
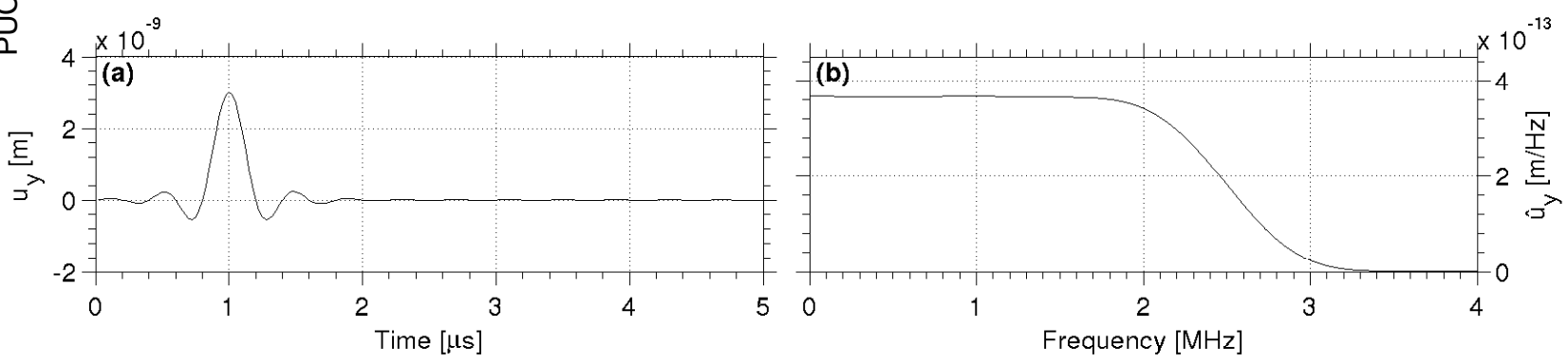

Figure 8.1: Wideband excitation signal. (a) Signal in time domain. (b) Signal in frequency domain.

Within this frequency band there are some possible Lamb modes able to propagate. These can be identified by observing the modes distribution in a dispersion curve chart, as the one shown in Fig. 2.3. For convenience, it is shown here the dispersion curves with the frequency-thickness scale tuned to 
a $3 \mathrm{~mm}$ thick plate in Fig. 8.2. The modes for which the cut-off frequency lies inside the excitation bandwidth consist up to the second order modes. The third order modes are almost outside the useful bandwidth, so very unlikely to be detect because their energy should be tiny and the group velocity of their frequency components very low.

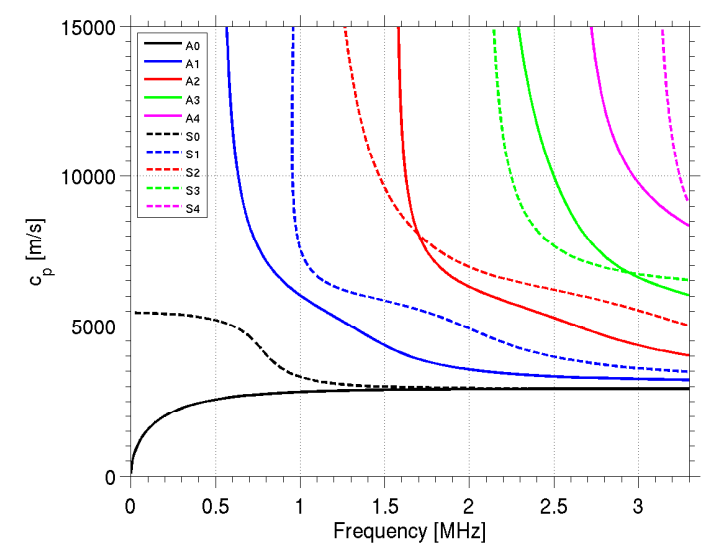

(a)

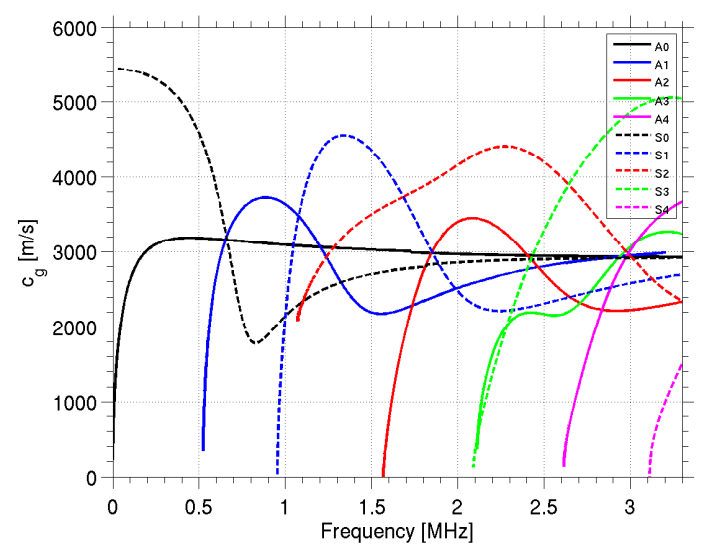

(b)

Figure 8.2: Phase (a) and Group (b) velocity dispersion curves for a $3 \mathrm{~mm}$ thick aluminum plate. Solid lines stands for antissymetrical modes and dashed lines for symmetrical. Black lines are fundamental modes, blue first order modes, red second order, green third order and pink forth order modes.

\section{1 \\ Infinitely long plate}

The propagated waves for the infinite plate (with absorbers) are seen in Fig. 8.3 as out-of-plane displacement at the top surface, in either grayscale map (a) and seismogram-like plot (b). The observable time window is up to $949.1 \mu \mathrm{s}$.

As it can be seen, the wave propagation is far more complex than presented in Fig. 6.4 and Fig. 6.8 due to the multi-mode propagation. In order to clearly identify the modes, the back propagation dispersion compensation technique proposed by Xu et al. [80] (explained in section 2.3.2) is used. The back propagation has been implemented for the signal sensed at $700 \mathrm{~mm}$ using the theoretical phase velocity curves. Back propagation was performed to all modes up to third order ones. The original received signal is shown in Fig. 8.4 and the dispersion compensated signals in Fig. 8.5.

The compensation is clear for the fundamental modes, where the peak at the origin $(t=0)$ is accentuated. The mode S1, A1 and S2 also compensate to an acute signal at the origin. The remainder modes do not compensate and their presence in the sensed signal is negligible. 


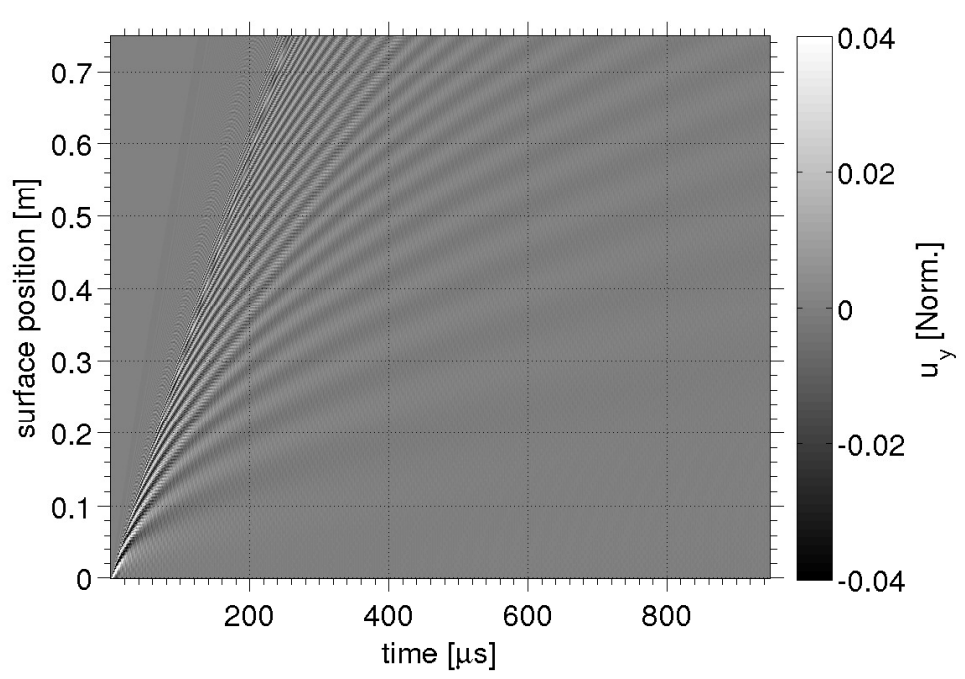

(a)

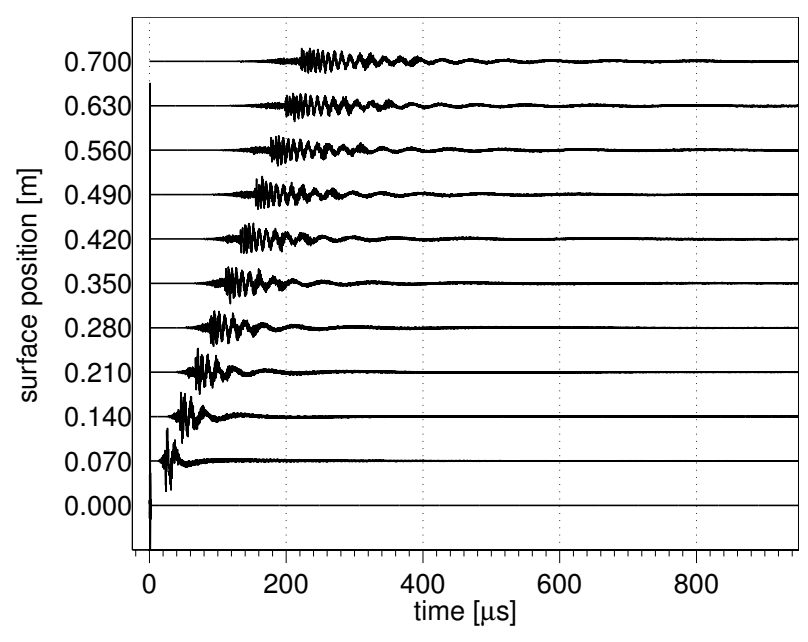

(b)

Figure 8.3: Wideband Lamb waves propagation sensed as out-of-plane displacement at top surface. (a) Grayscale map. (b) Seismogram-like plot.
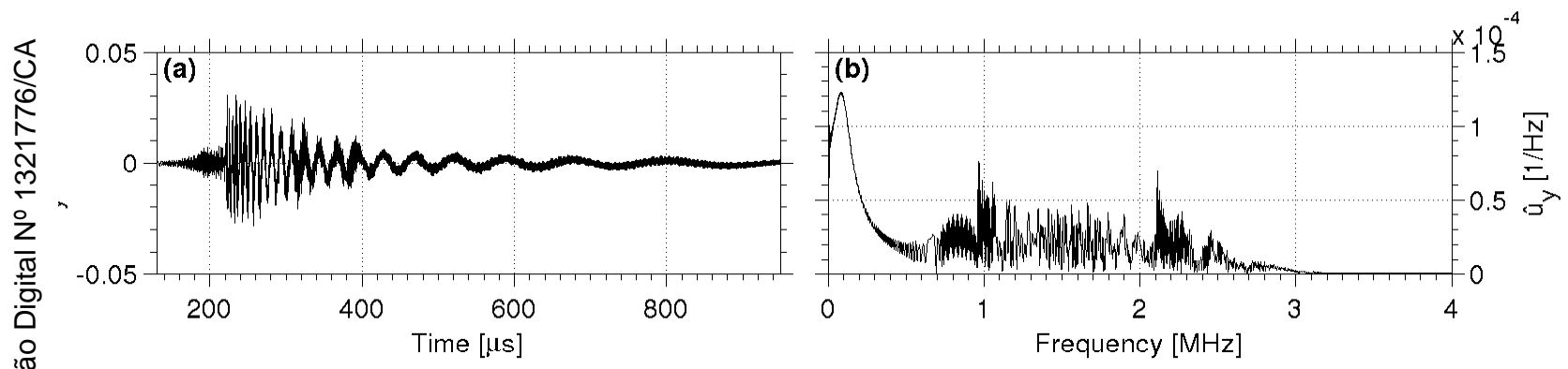

Figure 8.4: Wideband sensed signal at $x=700 \mathrm{~mm}$ in time domain (a) and frequency domain (b).

The signal sensed at $x=700 \mathrm{~mm}$ is used for a time-reversal simulation. For this purpose this signal (Fig. 8.4) is time reversed and set as the excitation signal for a new simulation. Only the time window from the first wavefront arrival ( $\mathrm{S} 0$ mode) is used for excitation, this correspond to the instant $t_{0}=$ $130 \mu \mathrm{s}$. The window length is $\Delta T=819.1 \mu \mathrm{s}$, resulting in a time window equal to the interval $t=[130 ; 949.1] \mu \mathrm{s}$. The resulting propagation is shown in Fig. 8.6, where it is observable the time-reversal focalization into a sharp pulse for exactly the position where the original signal has been collected at the instant equal to the temporal length of the original signal. That is, $x=70 \mathrm{~mm}$ and $t=949.1 \mu \mathrm{s}$, respectively. As predicted by the time-reversal theory exposed in section 7 . The signal at this position is detailed in Fig. 8.7, where three zoom levels around the focus are shown.

The correlation implementation of the time reversal technique is performed for the signal sensed at $x=700 \mathrm{~mm}$ (Fig. 8.4). This is done by convolving this signal with its time reversed version, as indicated in (7.9). This 
Chapter 8. Simulation of wideband time reversal of Lamb waves under longitudinal stress
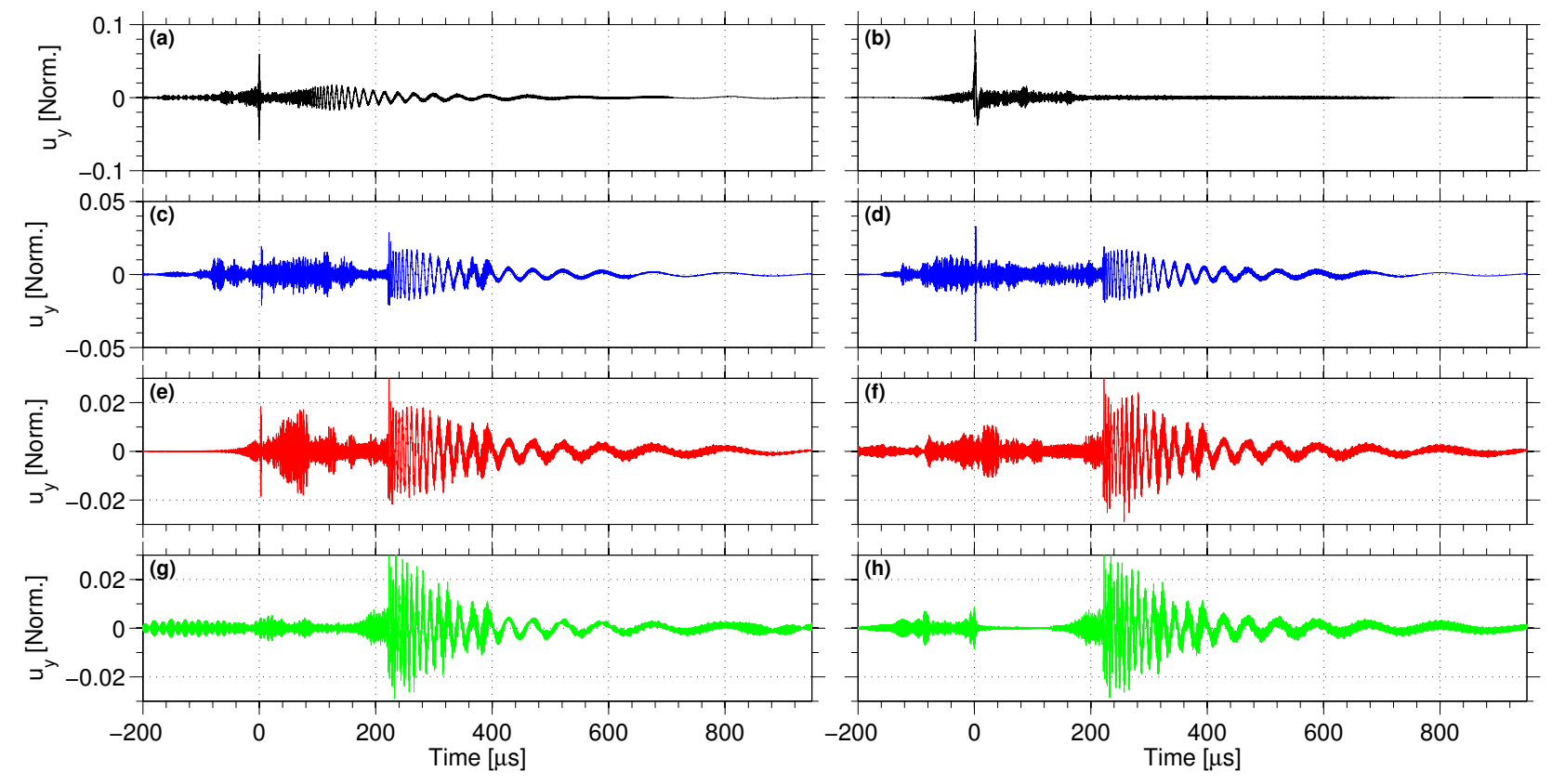

Figure 8.5: Dispersion compensation for fundamental to third order modes. (a)

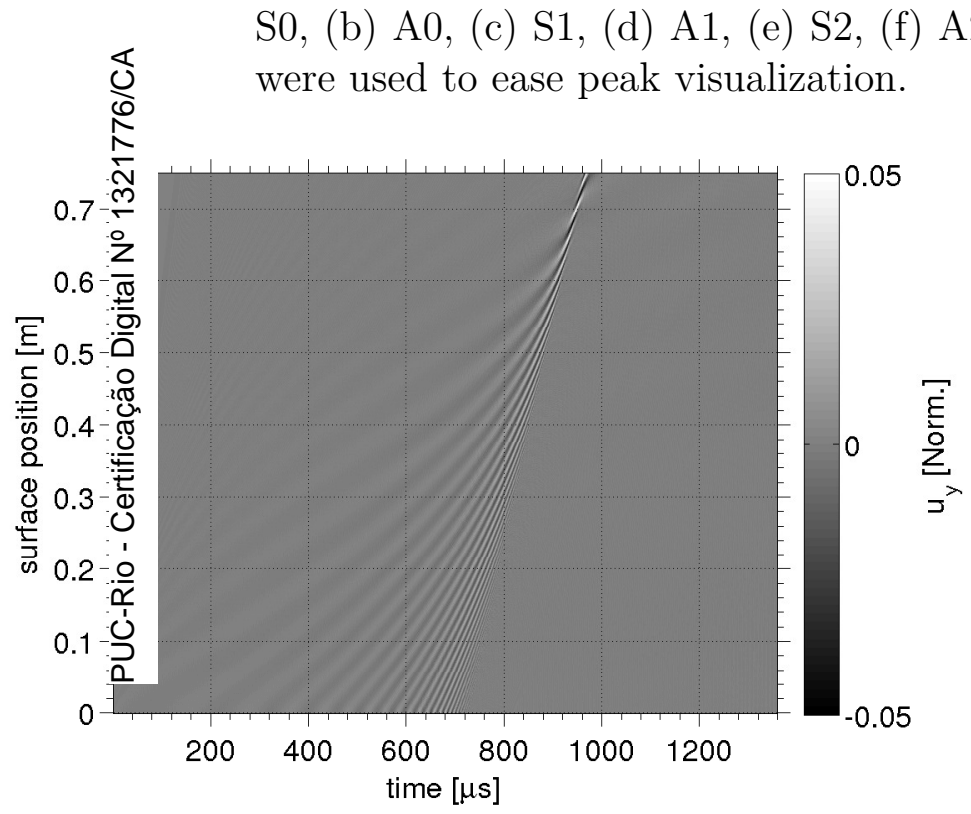

(a)

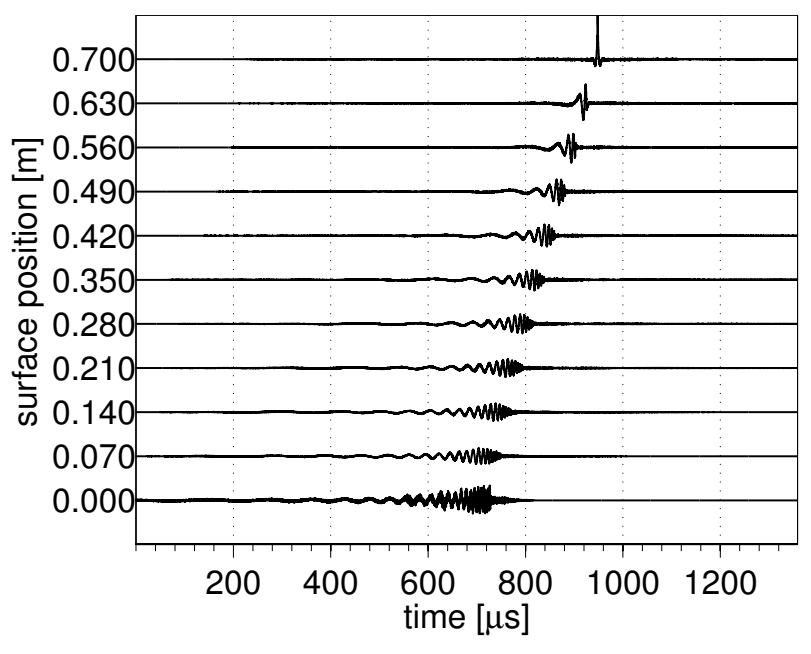

(b)

Figure 8.6: Wideband time reversal Lamb waves propagation sensed as outof-plane displacement at top surface. (a) Grayscale map. (b) Seismogram-like plot.

calculation was performed and shown in Fig. 8.7 together with the physical implementation, i.e., the simulation by means of time reversed excitation. As it can be seen, the overall behavior is the same despite existing some small differences, more clearly seen in the closest view (Fig. 8.7.c).

It is worth noticing the equivalence between both implementations by comparing some characteristics of the focus quality, for instance the peak and 
TREF values. These values are reported in Table 8.1 for both implementations, physical and correlation.

Table 8.1: Wideband time reversal focus characteristics.

\begin{tabular}{c|cc}
\hline Implementation & $\begin{array}{c}\text { Peak value } \\
(\text { Norm. })\end{array}$ & $\begin{array}{c}\text { TREF } \\
(\%)\end{array}$ \\
\hline Physical & 0.092 & 87.6 \\
Correlation & 0.085 & 87.8 \\
\hline
\end{tabular}
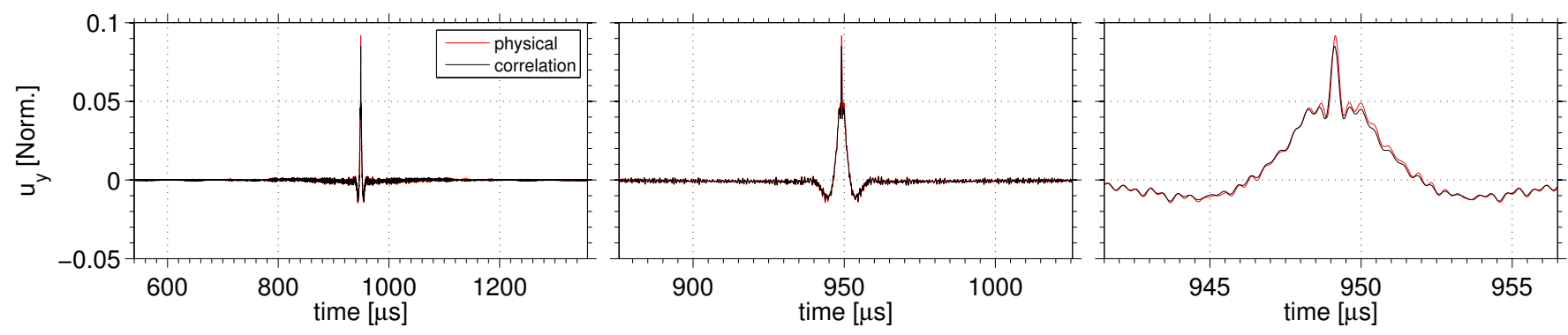

Figure 8.7: Wideband time reversal signal sensed at $x=700 \mathrm{~mm}$. Red line is the physical time reversal implementation and black line is correlation implementation, in three zoom levels.

\subsection{1}

\section{Effect of longitudinal stress on time reversal}

The effect of stress on the time reversal signal is simulated by modifying the medium stiffness according to EEC1 approximation of tensor $\mathbf{A}^{n}$. This implementation was validated in Chapter 6 for single mode Lamb waves. It is observed the time reversal signal at the focused position and instant. As in the examples above, the chosen position is $x=700 \mathrm{~mm}$. Fig. 8.8 shows the focused instant for some stress levels. Both physical and correlation implementations were performed for low stress values (up to 10MPa); for higher stress, only the correlation implementation was done. Both implementations use the signal received under null stress as reference. In the physical implementation this signal is time-reversed and then set as excitation into the modified medium, whereas in the correlation implementation this reference is post-processed with the received forward propagated signal from the modified medium.

As for the unstressed plate, the physical and correlation implementations present almost the same signals, both are sensible to stress. The two more evident characteristics are peak amplitude decrease and a peak time shift, as predicted in the theoretical section 7 . These characteristics are traced versus the stress level in Fig. 8.9. The peak decrease is reported related to the no-stress reference peak value for each implementation, respectively. For those stress 

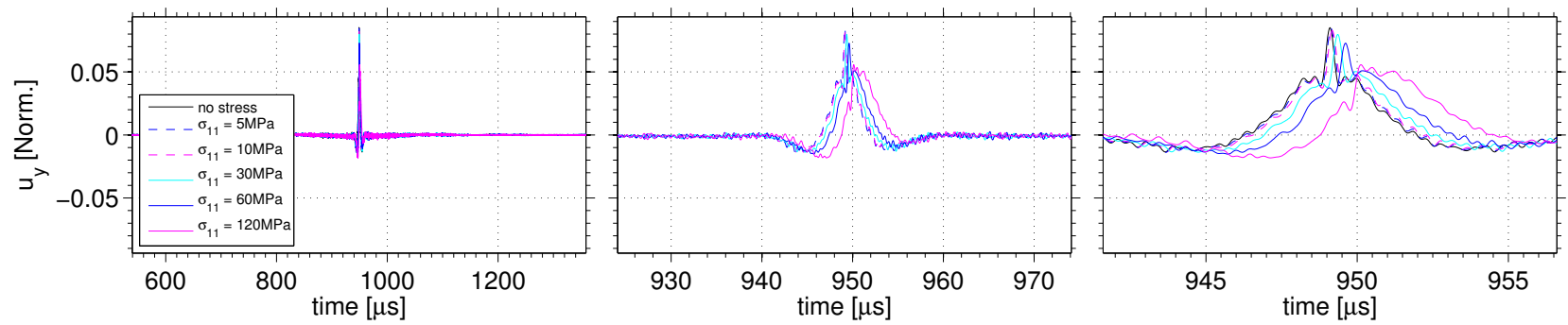

Figure 8.8: Wideband sensed focus at $x=700 \mathrm{~mm}$ for several longitudinal stress levels. Color stands for stress level.

levels where both implementations were simulated the difference between them are very small. The linear rate for the peak decrease is $-0.020 \% / \mu m / m$ and for the time-of-flight shift it is $0.60 \mathrm{~ns} / \mu \mathrm{m} / \mathrm{m}$ for the correlation implementation. The strain sensitive is reported in Table 8.3.

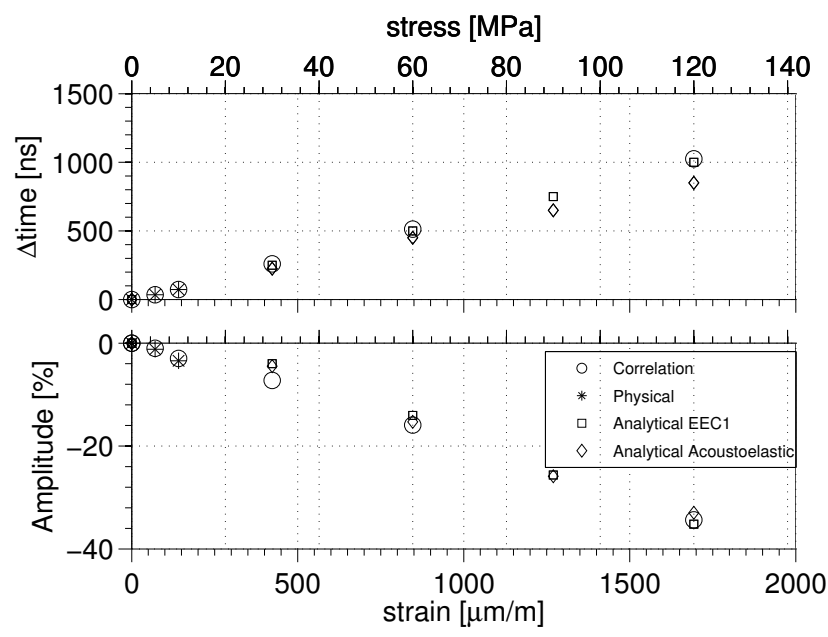

Figure 8.9: Time reversal focus behavior under stress at $x=700 \mathrm{~mm}$. Time shift (top) and amplitude relative decrease (bottom). The abscissa is either longitudinal strain, in $\mu \mathrm{m} / \mathrm{m}$ unit, or longitudinal stress, in $M P a$ unit.

The behavior for low and high stresses is about the same. Both present the same linear trend for peak time and value. However, one can easily conclude that a linear trend on peak decrease is not possible regardless the stress value, because it would imply in less than $100 \%$ of decrease, what makes no sense. There is, thus, a stress limit in which this measure can be used. In addition, if the peak value is far too low, then the time shift cannot be detected. Fig. 8.8 suggest that this limit is close to the highest stress imposed, since the peak of the highest stress response is almost mistaken by the side peaks presented in this signal. In this case, stress is so high that the focusing characteristic is almost lost. 


\subsubsection{1}

\section{Analytical model}

The expression (7.15) can be used to calculate the system transfer function as the sum of the modes. However, the amplitude of the modes as a function of the frequency need to be known. Here an approximation is introduced in order to investigate this model. The mode amplitudes are supposed to be constant along the frequency range but with different weights for each mode. These weights are estimated from the back propagated signals in Fig. 8.5. The amplitude of the peak around the origin of each mode in Fig. 8.5 is used as each respective weight.

The numerical propagation is performed for all modes. For this purpose, the original sinc signal (see Fig.8.1) is multiplied by the respective chosen weights, and then propagated to a distance of $700 \mathrm{~mm}$ using expression (7.15). Then all modes responses are summed. This is assumed as the system transfer function. The propagation is performed using the theoretical dispersion curves calculated in Chapter 5 for the unstressed medium and for longitudinal stress. Then, the response for the unstressed medium is time reversed and convolved with the stressed response, as indicates (7.14a). The resulted focused signals are shown in Fig. 8.10
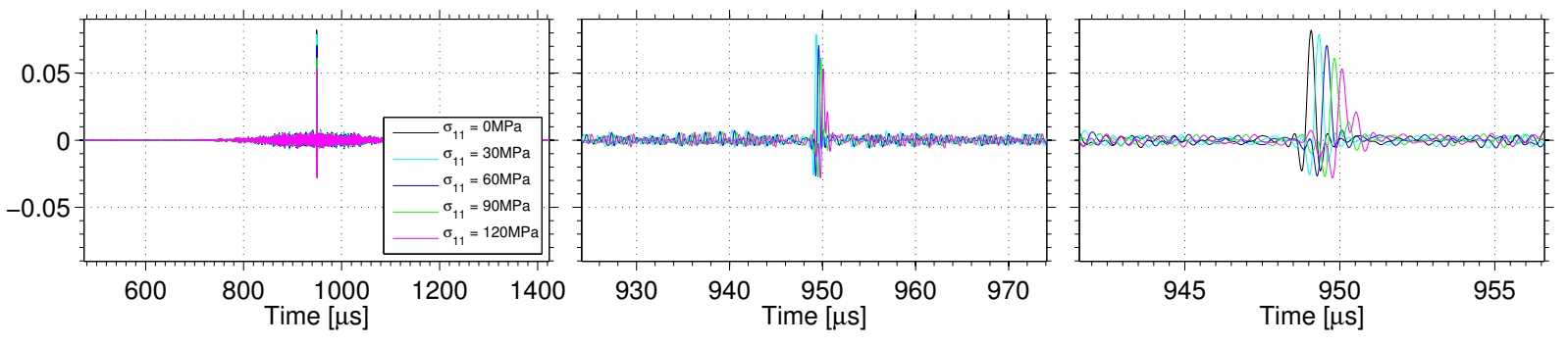

Figure 8.10: Wideband focus at $x=700 \mathrm{~mm}$ for several longitudinal stress levels calculated from analytical model. Color stands for stress level.

The main difference from the simulated waves relies in the assumption of flat spectrum for the modes. The simulated response by finite element distributes the initial pulse energy for all modes properly, according to the mechanical characteristics of the medium. In other words, the plate really acts as mechanical filter, implying the correct amplitude along the frequency spectrum. This is noticeable by comparing the focused signals in Fig.8.8 and Fig. 8.10. In the former, the system possesses high energy for the lower frequencies (see spectrum in Fig.8.3.b), and thus the focused signal present a larger focus spot compared to the latter.

Nevertheless, the stress effect is about the same because we tried to mimic the modes intensity. The peak value and its shift are reported in Fig. 8.9 with 
squares symbols. One can see that both measures coincide with the simulation. To be consistent with the simulation the model calculation was performed using the the dispersion curves obtained by means of EEC1 approximation.

In order to evaluate the effect of the EEC approximation on the timereversal process, the model was carried out with the exact dispersion curves. This is reported in Fig. 8.9 with diamond symbols. It is evident that the peak decrease is the same, whereas the time-shift is lower when using the exact dispersion curves. Recall that the theoretical errors in the phase velocity, reported in Fig. 5.21, are negative, and thus the velocity differences obtained with EEC1 are higher than the exact ones. Then, the global delay obtained by EEC1 should be greater than the one obtained by the exact dispersion curves. The time-of-flight shift rate for the EEC1 approximation is $0.59 \mathrm{~ns} / \mu \mathrm{m} / \mathrm{m}$; with exact dispersion it is $0.51 \mathrm{~ns} / \mu \mathrm{m} / \mathrm{m}$. The difference is $14 \%$, which is about the mean error on the sensitivity reported in Fig. 5.21. The peak decrease, on the other hand, present no difference. This is explained by the fact that the decrease on the amplitude is caused by the different phase sensitivities along the frequency range of each mode and between the modes, as predicted in section 7.4. A global difference on the sensitivity, as is the case for the EEC approximations, is relevant for the time-shift but introduces minor effects on the amplitude decrease.

\subsubsection{2 \\ Influence of sensing position}

The influence of the sensing position on the stress sensitivity is analyzed. The positions on the plate surface shown in the seismogram-like plot in Fig. 8.3.b are used for performing the time reversal experiment. For this, just the correlation implementation was performed. It is used a time window for reversion and excitation with the same length as the one used for the original experiment, i.e., $\Delta T=819.1 \mu \mathrm{s}$ for all positions. The initial time instant, $t_{0}$, is however set in order to assure detection of the first wave front, according to the following linear rule: $t_{0}=185.714 \times x \mu \mathrm{s} / \mathrm{m}$, where $x$ is the sensing position. The time reversal focused signal for some positions is shown in Fig. 8.11.

The focus overall shape seems to show no big difference. The focus characteristics reported in Table 8.1 are about the same, showing no trend with the sensing position. The focus sensitivity present however less preeminent changes for closer positions. This behavior is clearly shown in Fig. 8.12, where the sensitivities of peak decrease and shift versus the sensing positions are shown. It is observable a conclusive trend; the more distant is the sensing position, the higher is the sensitivity for both time-of-flight shift and amplitude. 

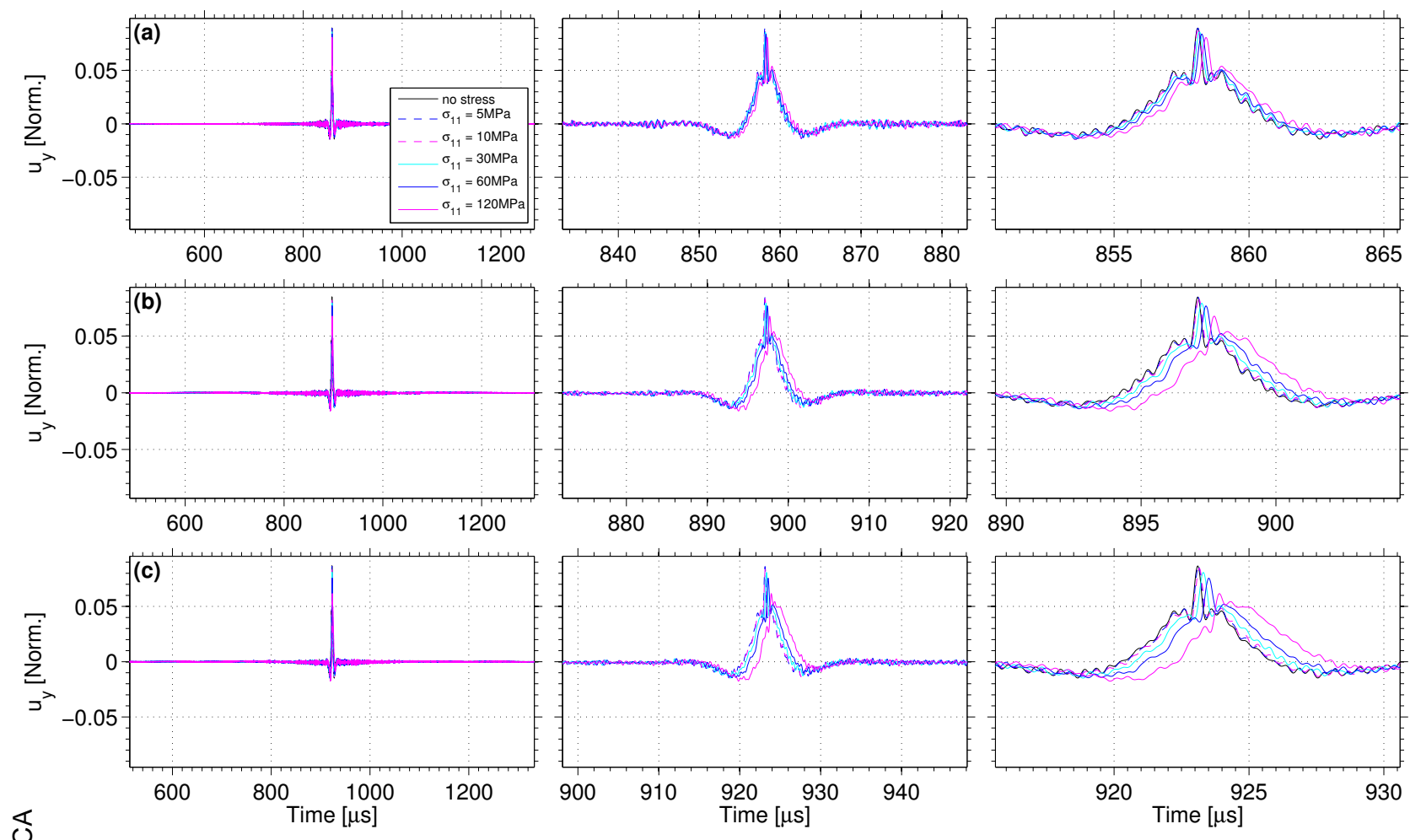

Figure 8.11: Wideband Lamb waves time reversal for several sensed position propagation in different zoom levels. (a) $x=210 \mathrm{~mm}$, (b) $x=420 \mathrm{~mm}$ and (c) $x=560 \mathrm{~mm}$.

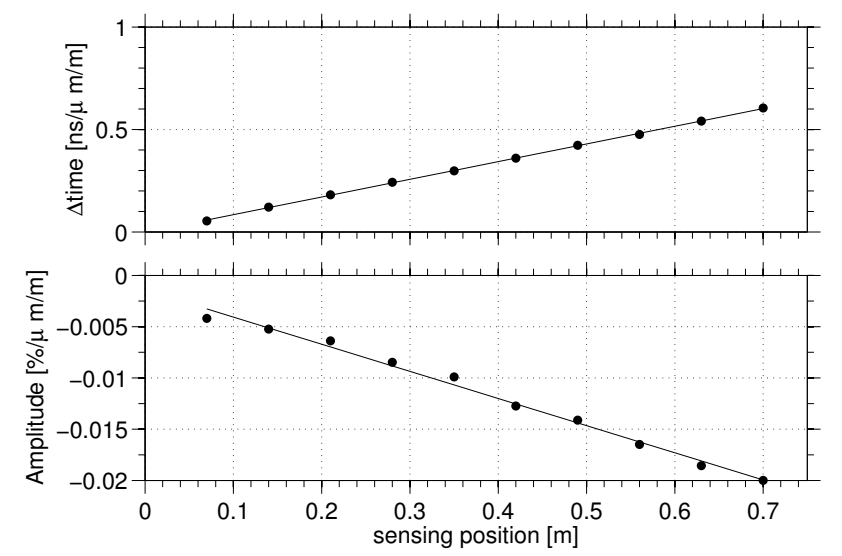

Figure 8.12: Wideband time reversal sensitivity of Lamb waves for several sensed position. Time-of-flight sensitivity (top) and Peak sensitivity (bottom).

The linear fit is superimposed with calculated sensitivities in Fig. 8.12. Their coefficients are $0.86 \mathrm{~ns} / \mathrm{m} / \mu \mathrm{m} / \mathrm{m}$ and $-0.0265 \% / \mathrm{m} / \mu \mathrm{m} / \mathrm{m}$ for time-offlight shift and peak decrease, respectively.

This time-of-flight shift rate can be compared with a group velocity sensitivity. For that a global group velocity is assumed as the sensed position divided by the peak time instant minus the transmitted signal length, i.e., 
$c_{g}^{T R}=x /\left(t_{\text {peak }}-\Delta T\right)$. The $\Delta T$ subtraction is necessary for discounting the time needed for transmitting the time-reversed excitation. Using this value into equation (5.10) one obtains the value of $-4.65 \times 10^{-} 6 / \mu \mathrm{m} / \mathrm{m}=-4.65 / \mathrm{m} / \mathrm{m}$. This value can be compared with the group velocity sensitivity chart in Fig. 5.7.b. For convenience the sensitivities are shown in Fig. 8.13 with the frequency axis tuned to $3 \mathrm{~mm}$ thick plate. As it can be seen this value is higher, in absolute value, than the sensitivity for low dispersive regions and lower than the high sensitivity regions, commonly at high dispersive regions (except for the $S 0$ mode at low frequency). This suggests that the sensitivity for the wideband time-reversal is composed by weighted contribution of each mode as indicated in (7.17).

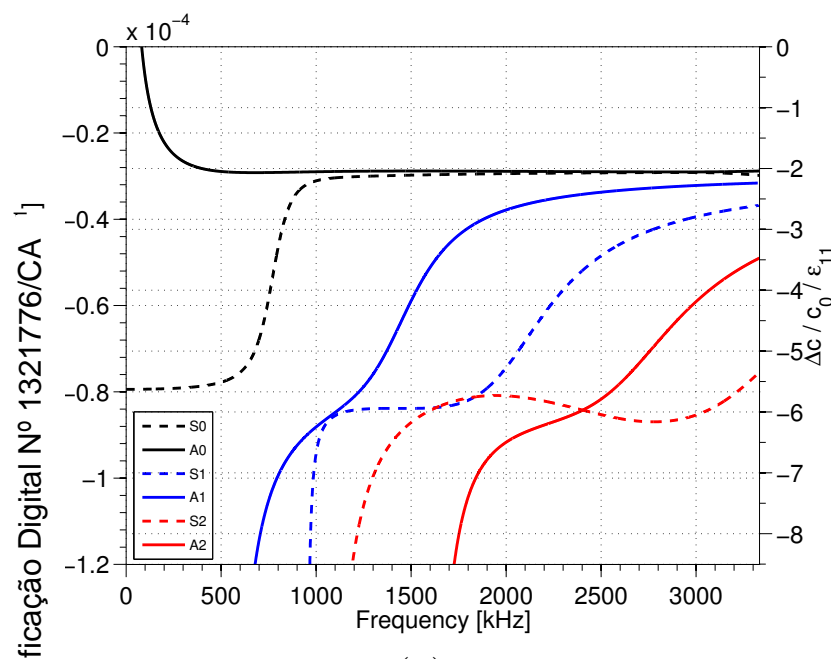

(a)

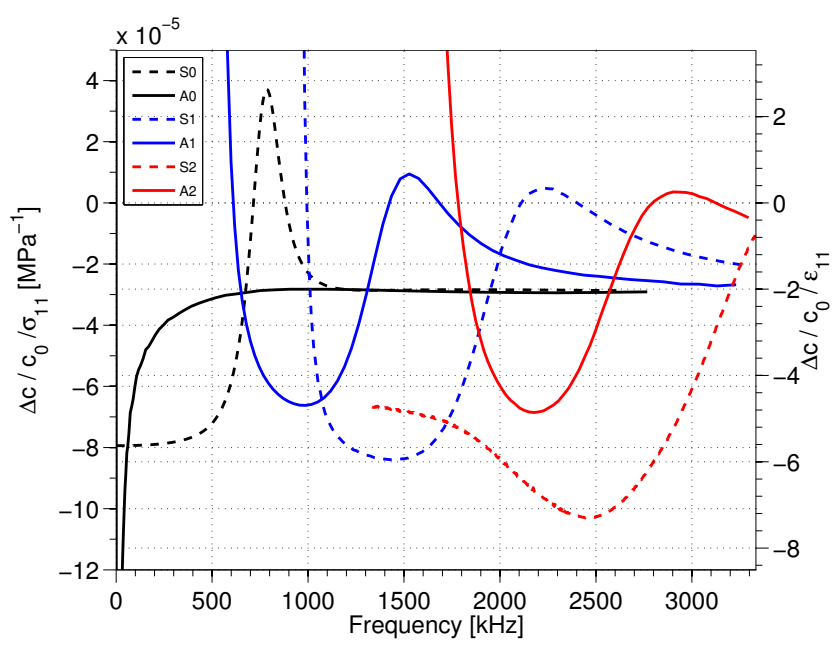

(b)

Figure 8.13: Acoustoelastic phase (a) and group (b) velocity sensitivity for up to second order modes at natural frame of reference for a $3 \mathrm{~mm}$ thick plate.

\subsubsection{3}

\section{Band limited propagation}

The focus characteristics and sensitivity are highly dependent on the signal bandwidth. In this section this fact is illustrated by passing the original sensed signal through some bandpass filter. Three different filter were used. The filter central frequency and bandwidth are reported in Table 8.2.

Table 8.2: Filter parameters.

\begin{tabular}{c|cc}
\hline $\begin{array}{c}\text { Filter } \\
\text { label }\end{array}$ & $\begin{array}{c}\text { Central Frequency } \\
(\mathrm{kHz})\end{array}$ & $\begin{array}{c}-6 \mathrm{~dB} \text { Bandwidth } \\
(\mathrm{kHz})\end{array}$ \\
\hline (a) & 438 & 326 \\
$(\mathrm{~b})$ & 690 & 187 \\
(c) & 883 & 371 \\
\hline
\end{tabular}


The filtered signals are shown in Fig. 8.14. It is possible to infer the modes contribution in each of these signals by observing their time-traces and confronting their spectra with the modes dispersion curves in Fig. 8.2.

For the signal filtered by filter (a), only the modes $A 0, S 0$ and $A 1$ can propagate because their cut-off frequency are inside the signal nonzero amplitude spectrum. The latter is only possible in a very low group velocity. However, observing the signal in the time domain, it is clear that the $A 1$ contribution is less preeminent because the oscillation for greater times (low group velocity) present very low amplitude. The $A 0$ mode contribution is evident as it is responsible for high amplitude just after $200 \mu s$, and the $S 0$ is the lower amplitude just before $200 \mu s$.

The filtered signal by filter (b) enhances the participation of mode $A 1$ by reducing the amount of energy in the low frequency region.

The last signal, due filter (c), has also mode $S 1$. This mode cut-off frequency is about $1000 \mathrm{kHz}$, where there is a high amplitude in the signal amplitude spectrum. At the time domain one can see the strong wave front at about $400 \mu s$ followed by long tail due to this mode low group velocity around the cut-off frequency.
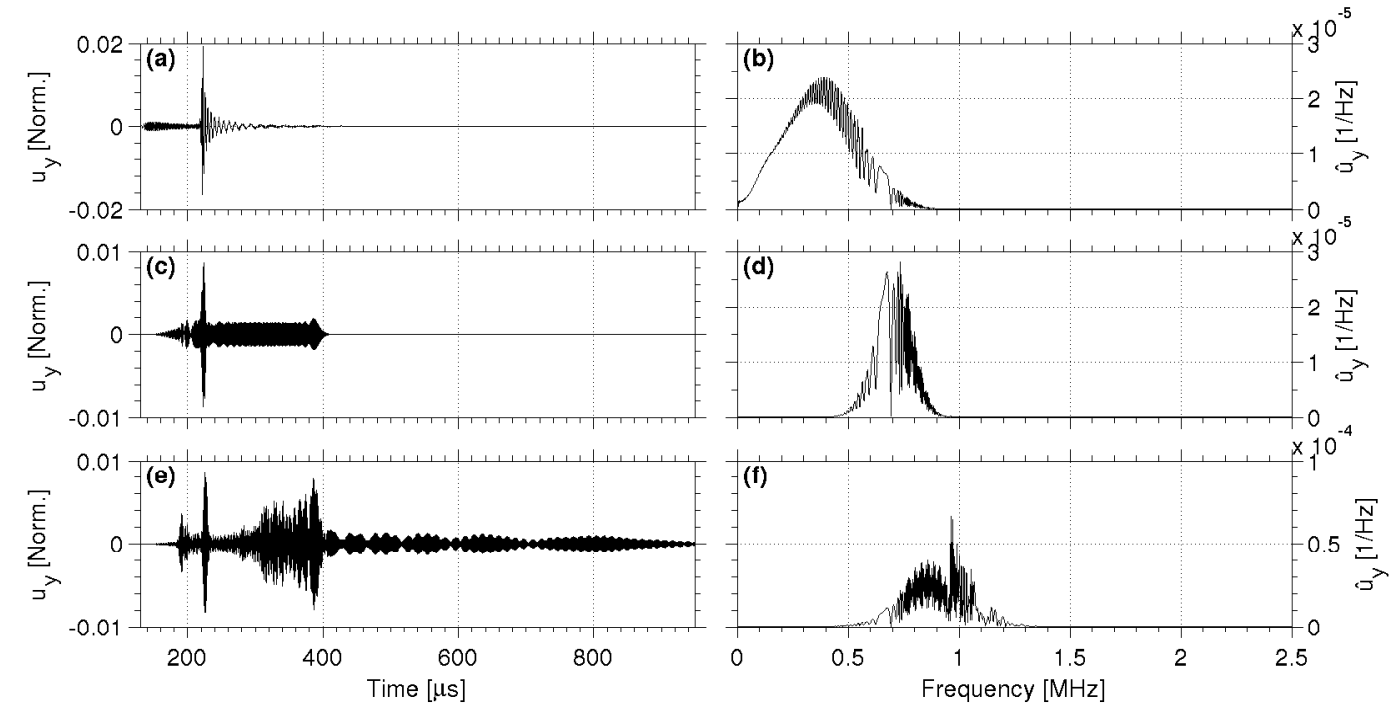

Figure 8.14: Filtered signals in time domain (left) and their Fourier transform (right) by filter (a), figures (a) and (b); filter (b), figures (c) and (d); and filter (c), figures (e) and (f).

The time reversal process is performed with the filtered signals, either by the correlation process or by the physical implementation. The correlation implementation was done by filtering the reference signal for null stress condition and the signal related to the stressed medium. Then the reference signal is time reversed and both are correlated. The physical implementation is done by filtering the signal twice. This is necessary because the focused signal 
is the result of the reference signal passed through the system. Thus if one wishes to reproduce the effect of using a band limited signal, due to filtering, one must filter the physically obtained focused signal twice. The time reversal signals are shown in Fig. 8.15.
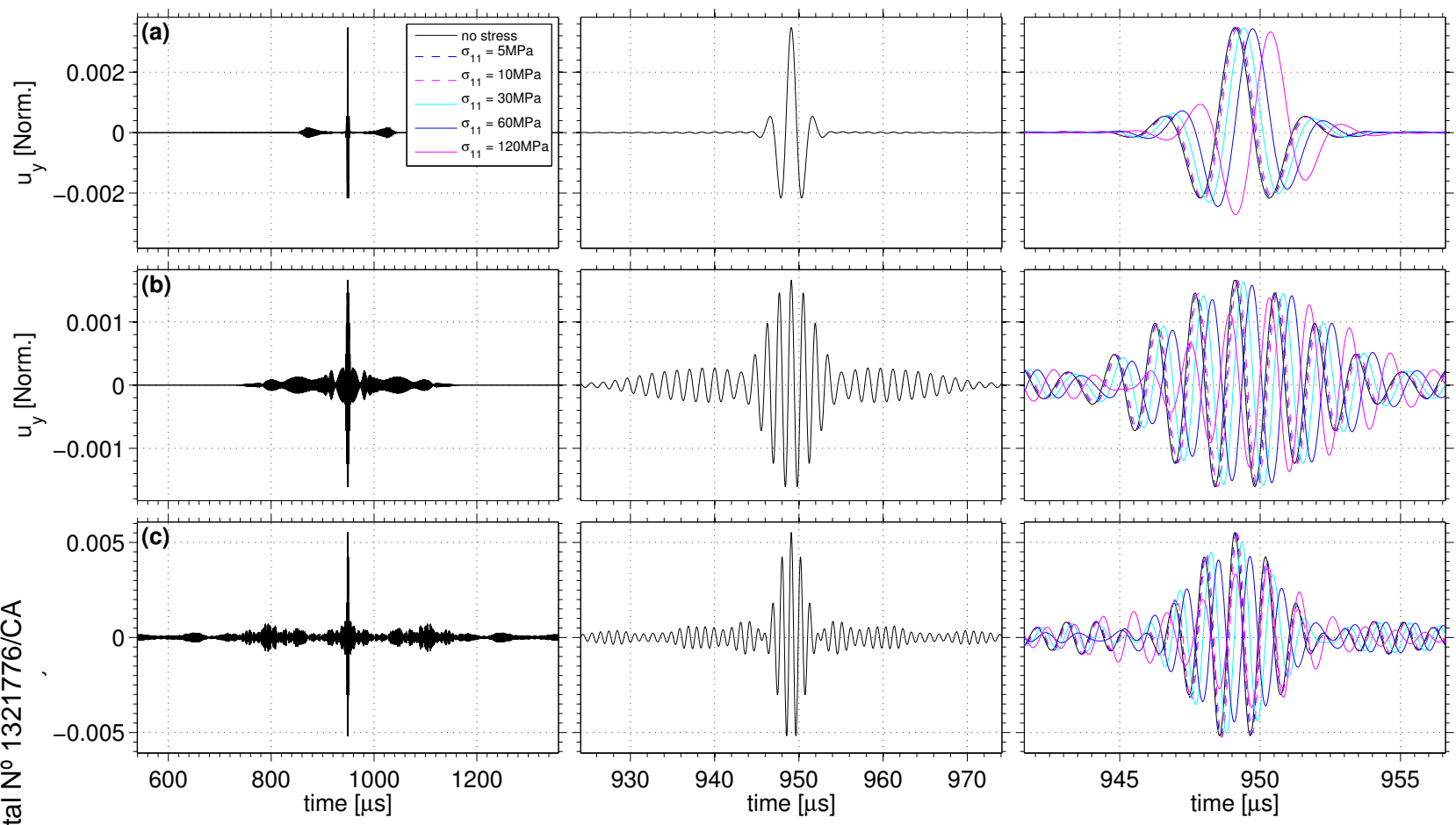

Figure 8.15: Filtered time reversal signals by filters (a), (b) and (c), respectively, in three zoom levels.

The focus shape and its sensitivity differ for each filter. In order to ease interpretation of the peak amplitude and of the time of arrival behaviors, these are shown in Fig. 8.16.a for low stress range, and in in Fig. 8.16.b for high stress range.

Observing the low stress behavior, one may conclude that there is a slight trend in the time-of-flight delay. The greater the amount of modes, the lower is the delay. The peak decrease behaves in the opposite direction: the greater the amount of modes, the higher is the peak decrease. This is as predicted in Chapter 7 .

This last figure also highlights the fact that the behavior of the peak decrease is different for low and high stresses. This is specially clear for the filtered signals where the value for high stresses is visibly not in a straight line (observe how signal (c) evolves in Fig. 8.16.b compared to the raw signal).

For the low stress range, the angular coefficient of a linear fit for peak decrease and time-shift were used to compare the results as a mean sensitivity. These rates are reported in Table 8.3. 


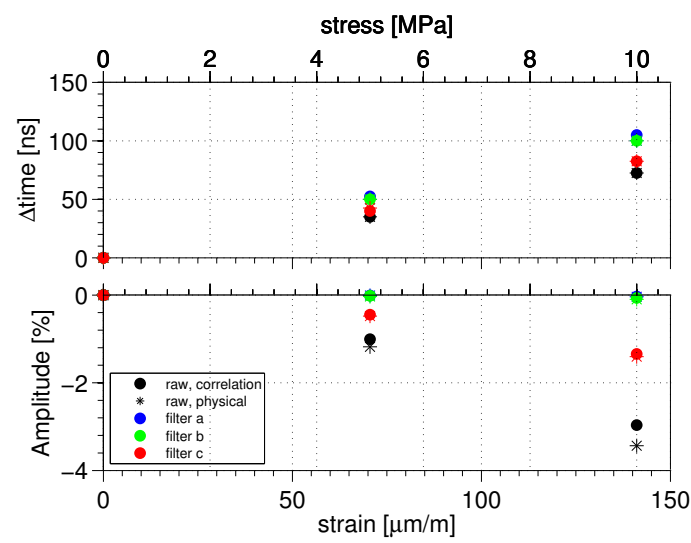

(a)

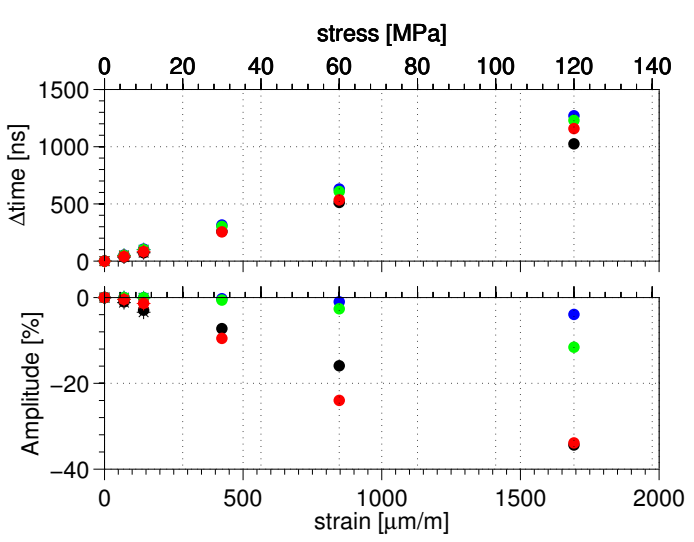

(b)

Figure 8.16: Time reversal focus behavior for filtered signals. Time shift (top) and amplitude relative decrease (bottom). The abscissa is either longitudinal strain, in $\mu \mathrm{m} / \mathrm{m}$ unit, or longitudinal stress, in $M P a$ unit. Black points stand for raw signal. (a) Low stress range. (b) High stress range.

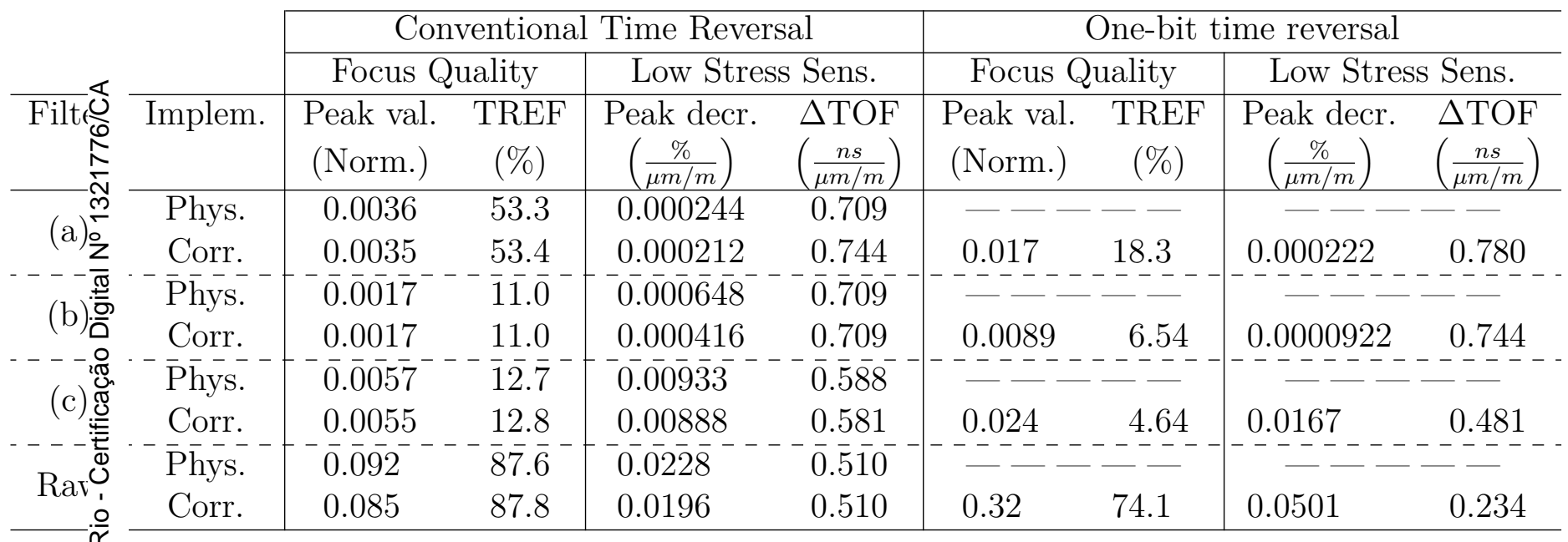

Table 8.3: Filtered time reversal focus and sensitivity characteristics, conventional and one-bit.

\subsubsection{4}

\section{One-Bit Time Reversal}

The one-bit time reversal, explained in 7.5, as a possible simplification for the technique is analyzed here. This was done only in the correlation implementation by taking the sign of the time-reversed reference signal before the correlation, as indicated by (7.18). The amplitude of the one-bit signal is set to the maximum of the signals received, i.e., the amplitude $V$ in (7.18), is set as $V=\max \left\{\left|y_{0}(t)\right|\right\}$. Fig. 8.17 shown the one-bit time-reversed version of the raw signal, which is shown in Fig. 8.4 to illustrated the process.

The resulting time reversal signal obtained by using the the one-bit excitation is shown in Fig. 8.18 for the raw signal and in Fig. 8.19 for the filtered signals, presented in the previous section. 

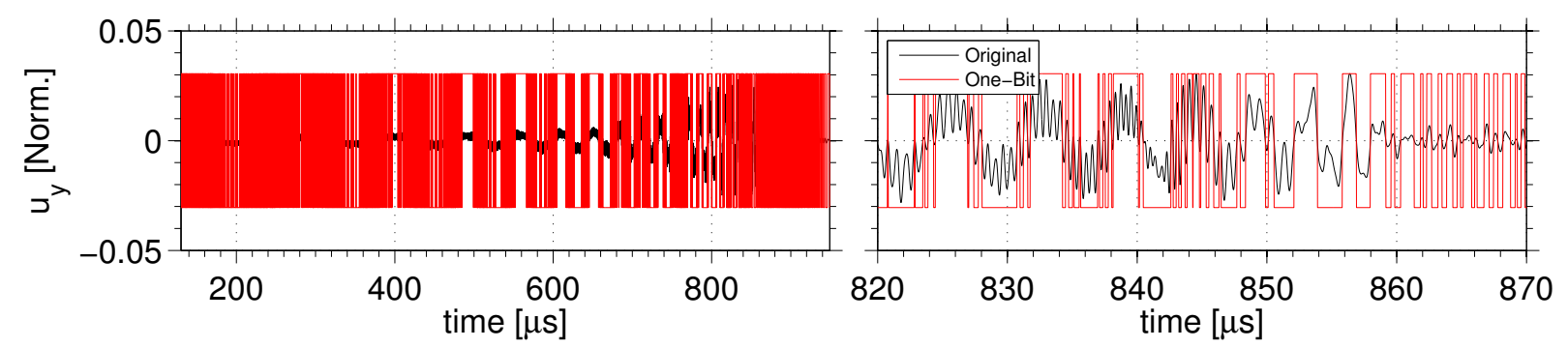

Figure 8.17: Wideband one-bit time-reversed signal. Full length reversed signal (left) and zoom for a $50 \mu \mathrm{s}$ time window (right).
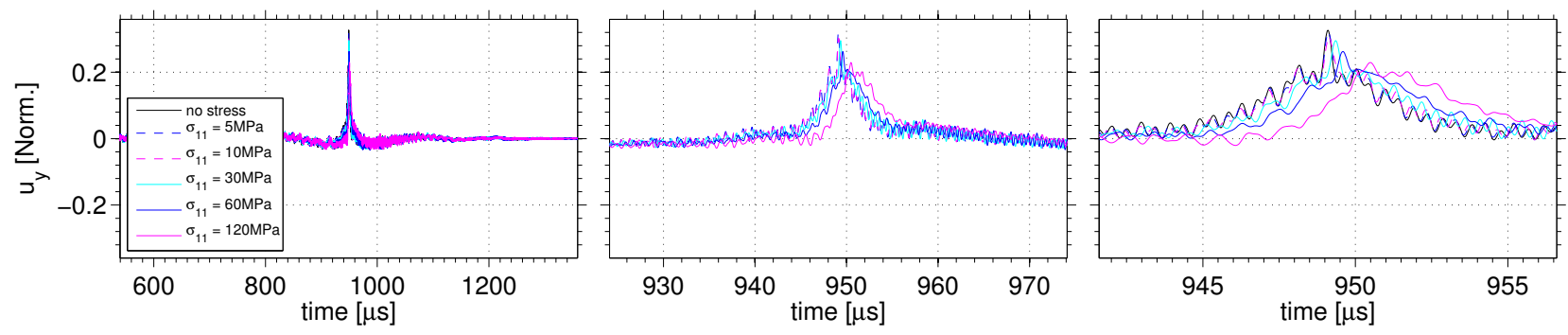

Figure 8.18: Wideband One-Bit Time Reversal signal, in three zoom levels.

The effect of the one-bit excitation is noticeable by comparing Fig. 8.8 with Fig. 8.18. The most evident effects are lack of symmetry, higher energy outside the focus, and a considerable amplitude gain on the focus, 3.48 times, as predicted in the theory of section 7.5. The degradation on the focus can be measured by the TREF value, which, due to the loss of information in the one-bit procedure, is lower than in the conventional time reversal. The focusing characteristics are reported in Table 8.3.

The effect of the one-bit time reversal on band-limited signals are more severe. One may see that the signal shapes in Fig. 8.19 are far from the ones in Fig. 8.15. The TREFs are less than half of the conventional values, see Table 8.3; the peak values is always higher.

The stress effect does not show an homogenous trend for the one-bit variation. Fig. 8.20 shows the peak and time of flight shift. Filter (a) and (b) present a very low amplitude reduction, almost constant behavior, whereas in the filter (c) and raw signal amplitude decrease increases. Table 8.3 reports the linear rates for the low stress range.

The effect of high stress is so intense that signals may not match at all, depending on the spectral content, as is the case of filter (c), shown in Fig. 8.19.c. For this, the peak instant for high stress is before the peak instant for no stress, i.e., it presented negative time-shift, and its point is not visible in Fig. 8.20.b. This illustrates the fact that if the peak decrease is too intense then determination of its instant may be compromised. 

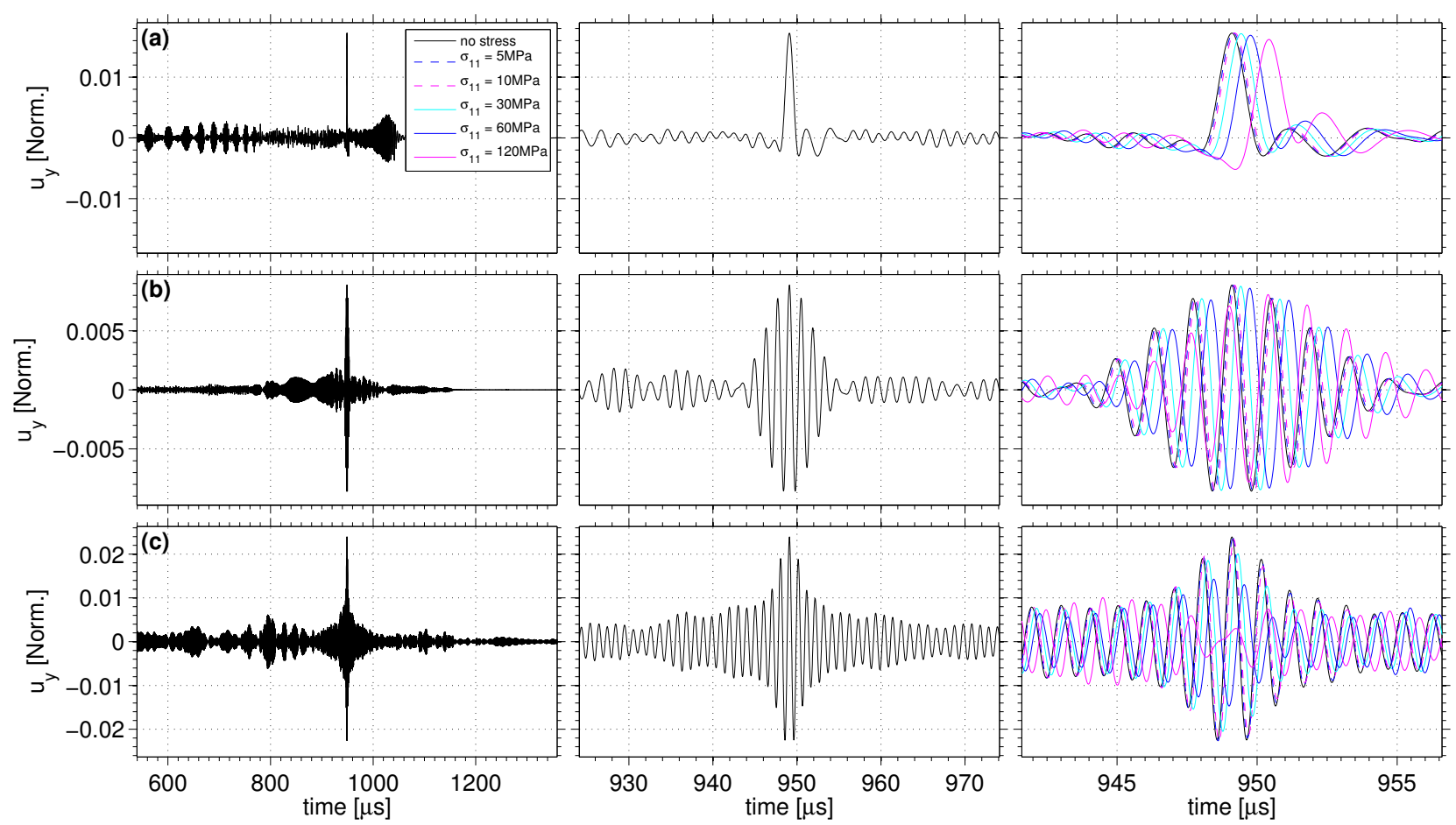

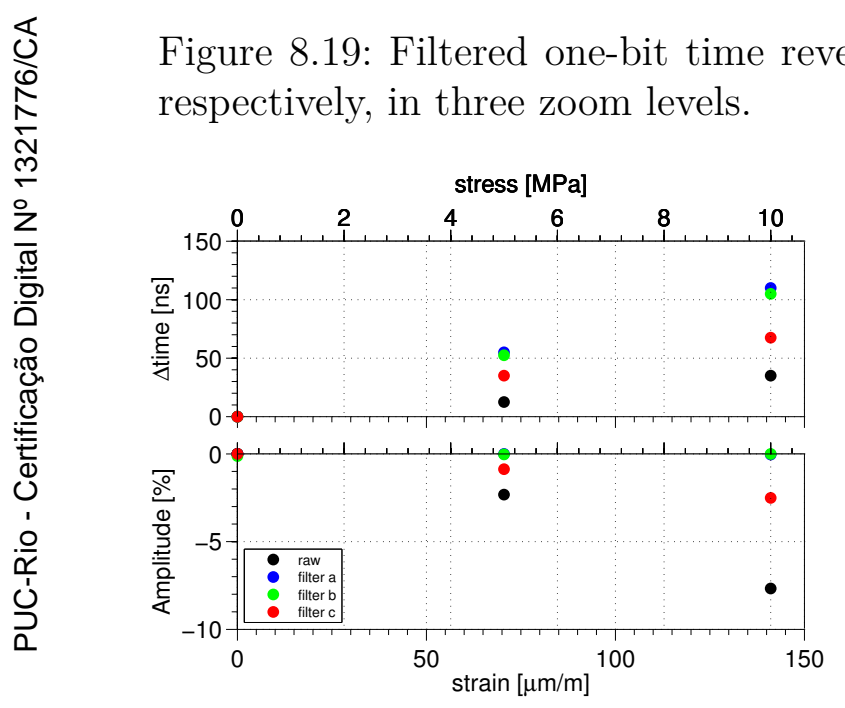

(a)

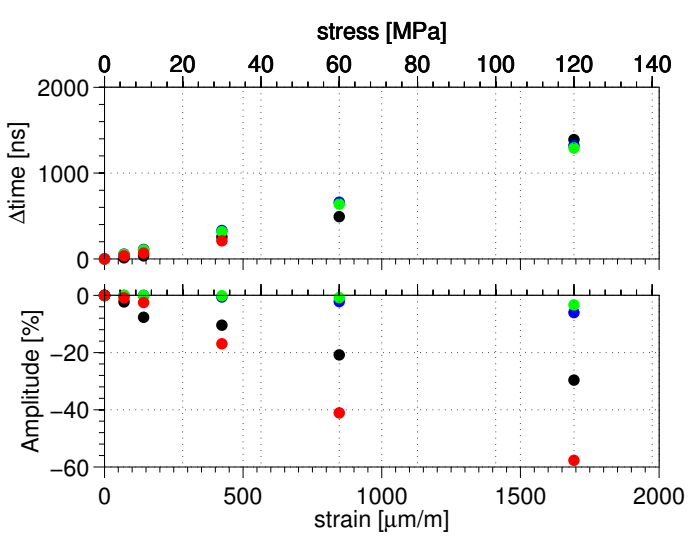

(b)

Figure 8.20: One-bit time reversal focus behavior for filtered signals. Time shift (top) and amplitude relative decrease (bottom). The abscissa is either longitudinal strain, in $\mu \mathrm{m} / \mathrm{m}$ unit, or longitudinal stress, in $M P a$ unit. Black points stand for raw signal. (a) Low stress range. (b) High stress range.

\section{2}

\section{Finite length plate}

In this section the propagation in a finite length plate is analyzed. Its geometry is the one presented in Fig. 6.1 without the absorber, thus from $x=-50 \mathrm{~mm}$ to $x=750 \mathrm{~mm}$. Excitation is still applied at the origin. The propagated waves are shown in Fig. 8.21. The reflections are evidently observable. As no damping has been included in the simulation, the reflected 
Chapter 8. Simulation of wideband time reversal of Lamb waves under longitudinal stress

echoes seem to present about the same intensity, differently from what should be expected in a real experiment, where the wavefronts that travel longer distance are weaker, due to attenuation.

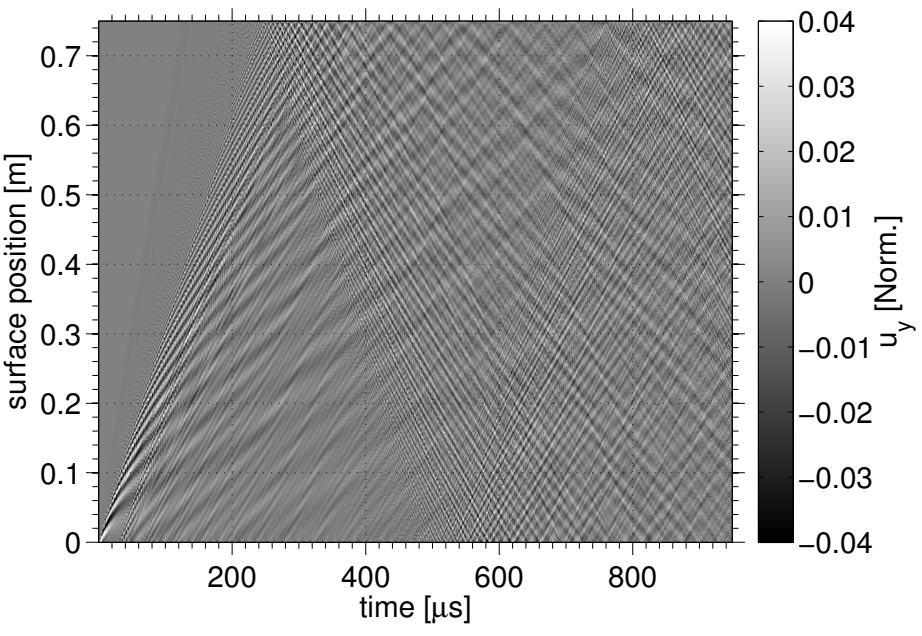

(a)

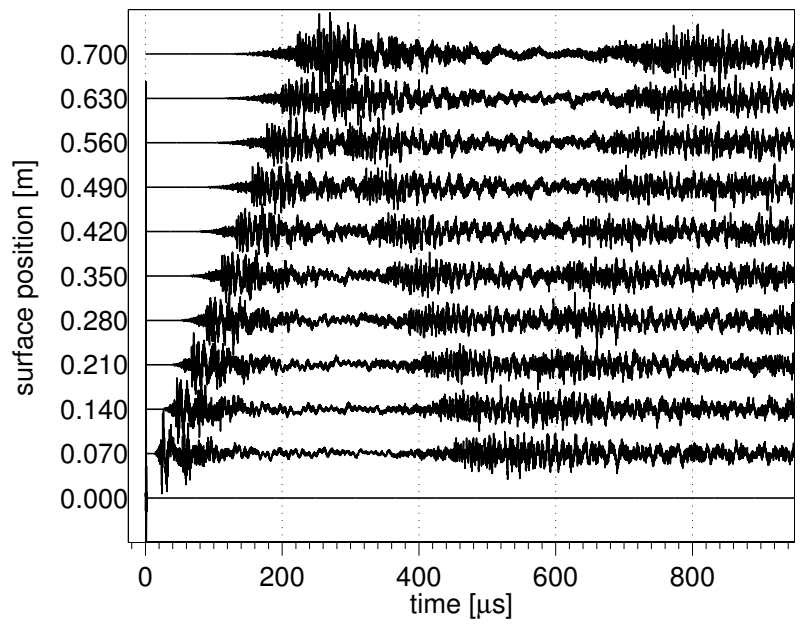

(b)

Figure 8.21: Wideband Lamb waves propagation in finite plate sensed as outof-plane displacement at top surface. (a) Grayscale map. (b) Seismogram-like plot.

The sensed signal at $x=700 \mathrm{~mm}$ is shown in Fig. 8.22. The propagation response to the time reversed excitation is shown in Fig. 8.23.
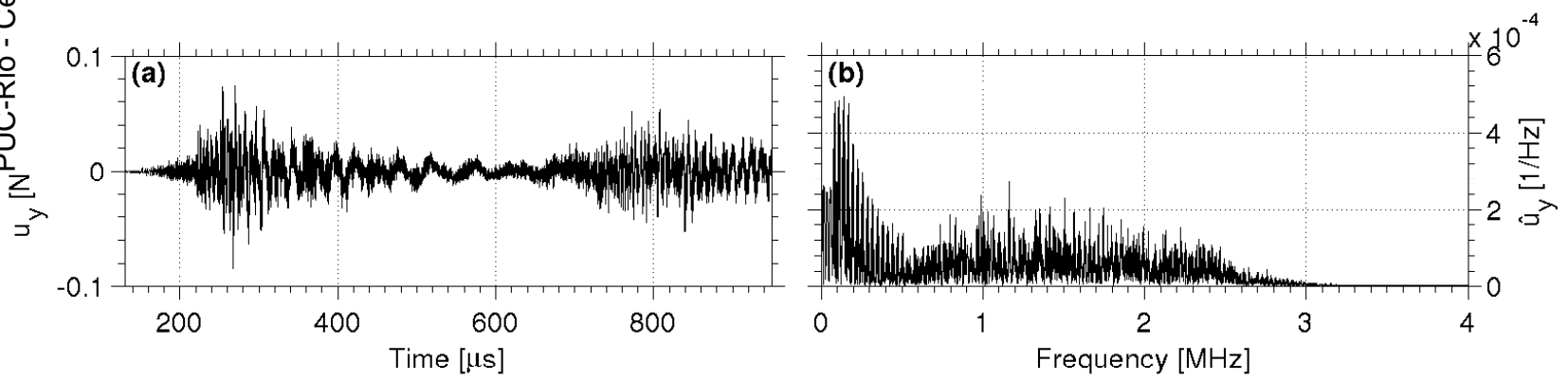

Figure 8.22: Wideband sensed signal at $x=700 \mathrm{~mm}$ finite length plate in time domain (a) and frequency domain (b).

The signal detailed at the focus position $(x=700 \mathrm{~mm})$, in both physical and correlation implementations, is shown in Fig. 8.24. The focus characteristics are reported in Table 8.4. Again both implementations are practically equal.

Comparing this focused signal with the one obtained for the infinite length plate (Fig. 8.7), two main characteristics are evident. First, for the finite length medium there are considerable side lobes in focused signal, which makes 


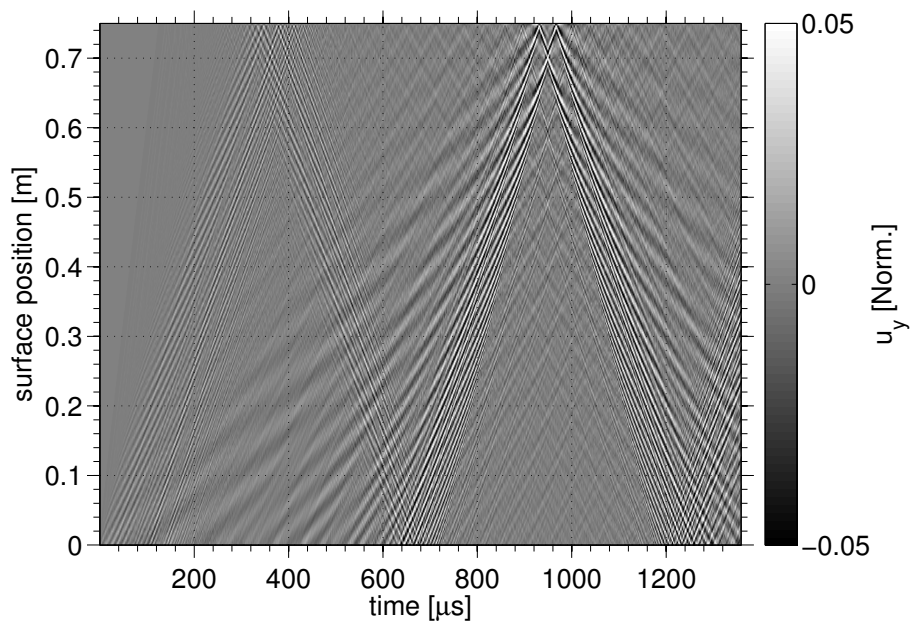

(a)

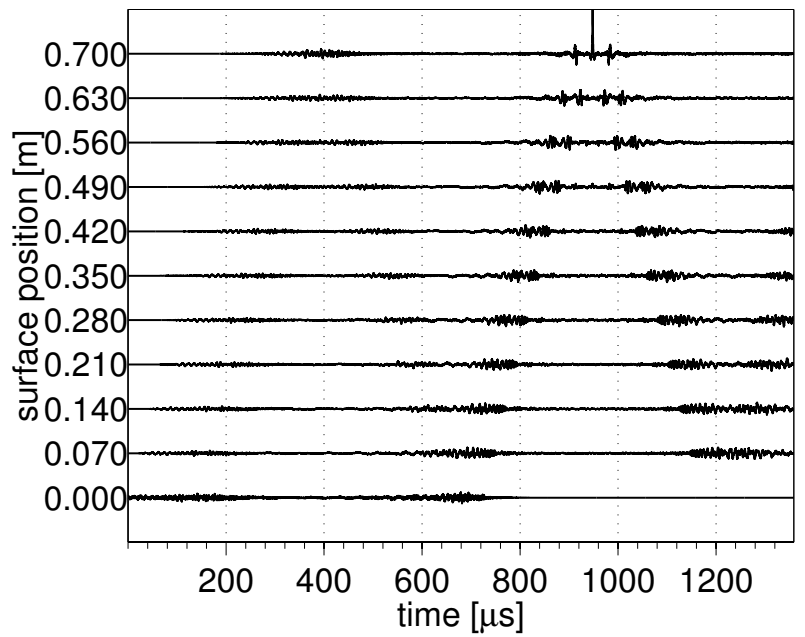

(b)

Figure 8.23: Wideband time reversal Lamb waves propagation for finite length plate sensed as out-of-plane displacement at top surface. (a) Grayscale map. (b) Seismogram-like plot.
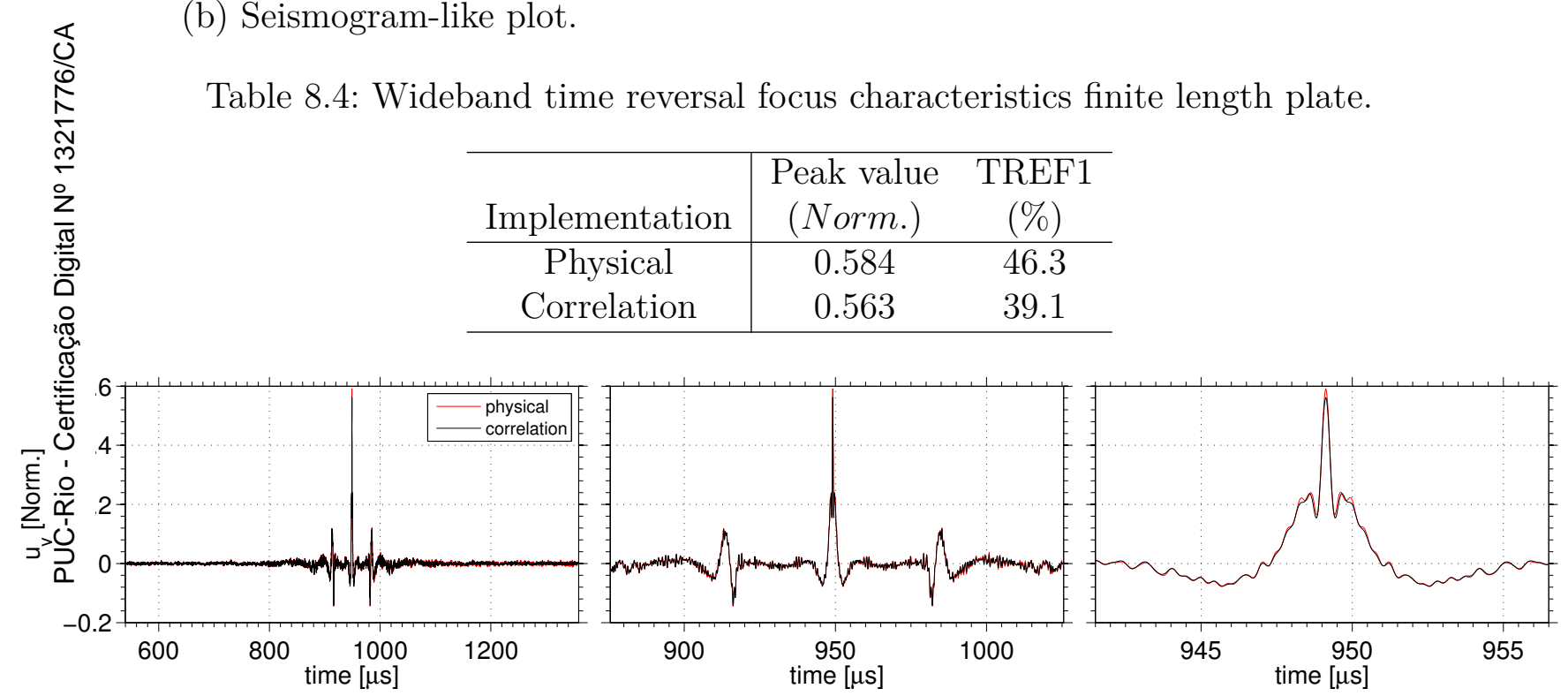

Figure 8.24: Wideband time reversal signal sensed at $x=700 \mathrm{~mm}$ for finite length plate. Red line is the physical time reversal implementation and black line is correlation implementation in three zoom levels.

TREF to be considerable lower. Second, the focused peak for the finite length plate, where there are reflections, is higher than for the infinite length plate (6.42 times in the physical implementation and 6.60 times in the correlation one). In the infinite medium the wavefront passes the sensing position and continues indefinitely (actually, the present implementation absorbs it). On the contrary, for the closed medium (finite length plate) each propagated wave is confined in the plate ad infinitum, as no damping has been simulated. Thus 
all the initial energy is kept inside the system and must be found at the focused signal.

\subsection{1}

\section{Effect of longitudinal stress on time reversal}

As done for the infinite plate, the stress effect on the focusing capability of the time reversal process is investigated for the finite length plate. Fig. 8.25 shows the focused instant for some stress levels at position at $x=700 \mathrm{~mm}$.
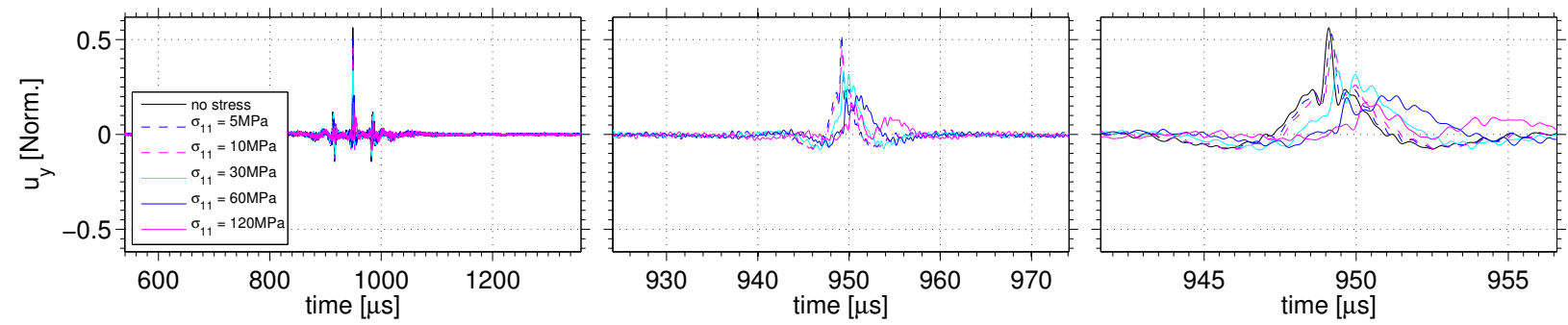

Figure 8.25: Wideband sensed focus at $x=700 \mathrm{~mm}$ for several longitudinal stress levels in a finite plate. Dashed lines are physical time reversal implementation, straight lines correlation implementation. Color stands for stress level.

Comparing this behavior with the focusing degeneration due to stress in the infinite medium (Fig. 8.8), it is noticeable that the effects are more severe in this latter scenario. This is even more visible in Fig. 8.26, where peak decrease and time-of-flight shift are shown as a function of strain. For higher loads the signal is already degenerated and peak measurement is compromised. The linear rates for low sensitivity are $-0.130 \% / \mu \mathrm{m} / \mathrm{m}$ for the peak decrease and for the time-of-flight shift it is $1.11 \mathrm{~ns} / \mu \mathrm{m} / \mathrm{m}$ in the correlation implementations. Much higher than in the infinite plate.

The higher sensitive obtained for the finite length plate is due to the higher complexity of the medium. In the first case the only sources of mismatches are the several propagating modes that are differently affected by stress, due to the different sensitivity of each of them, as theoretically demonstrated in Chapter 4. While for the closed medium the time reversal process must match not only the direct propagated modes but also all the reflected echoes from both ends. Thus it become more sensitive to the external perturbations, in this case, the stress. 


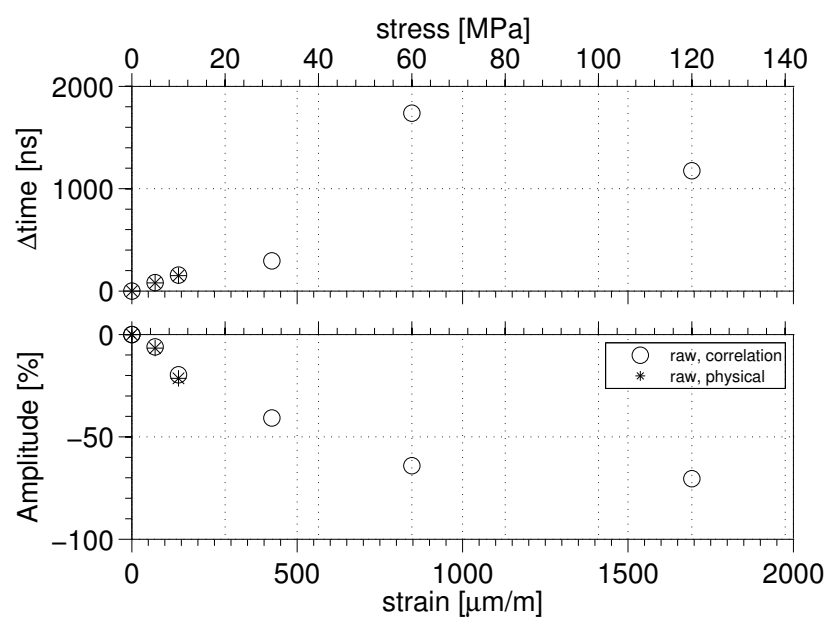

Figure 8.26: Time reversal focus behavior under stress at $x=700 \mathrm{~mm}$ in a finite plate. Time shift (top) and amplitude relative decrease (bottom). The abscissa is either longitudinal strain, in $\mu \mathrm{m} / \mathrm{m}$ unit, or longitudinal stress, in $M P a$ unit.

\subsubsection{1}

\section{Influence of sensing position}

The influence of sensing position for the time reversal sensitivity to stress in the finite length plate is analyzed by means of the correlation implementation. The focused signal for some positions is shown in Fig. 8.27.

The time-of-flight and peak decrease sensitivity is shown in Fig. 8.28 for the low stress range. The behavior for the finite plate is completely different from the infinite plate (Fig. 8.12). Initially, one can see that for any position both sensitivities (time-of-flight shift and amplitude decrease) are more intense in the finite plate than in the infinite one, at least $84.3 \%$ for time-of-flight and $400 \%$ for amplitude. Differently from the infinite medium, the time-of-flight increases a just little with the position, but not linearly, and the peak decrease is almost constant for all positions.

This indicates that the sensing position is not relevant for a finite plate as, generally speaking, for all positions the same amount of information due to direct and reflected wave is received. If a position is closer to the emitter, and should be less sensitive to the direct received waves, then it presents a longer path for the reflected waves, and consequently higher sensitivity for reflected waves. In other words, the overall sensitivity remains nearly constant, regardless the distance between emitter and receiver.

In this closed medium, the time length of the signal used for the timereversed excitation would be a possible source of influence for the focusing quality and its sensitivity. The longer the time window, more echoes are detected and can participate in the focusing process. This effect in the focusing 

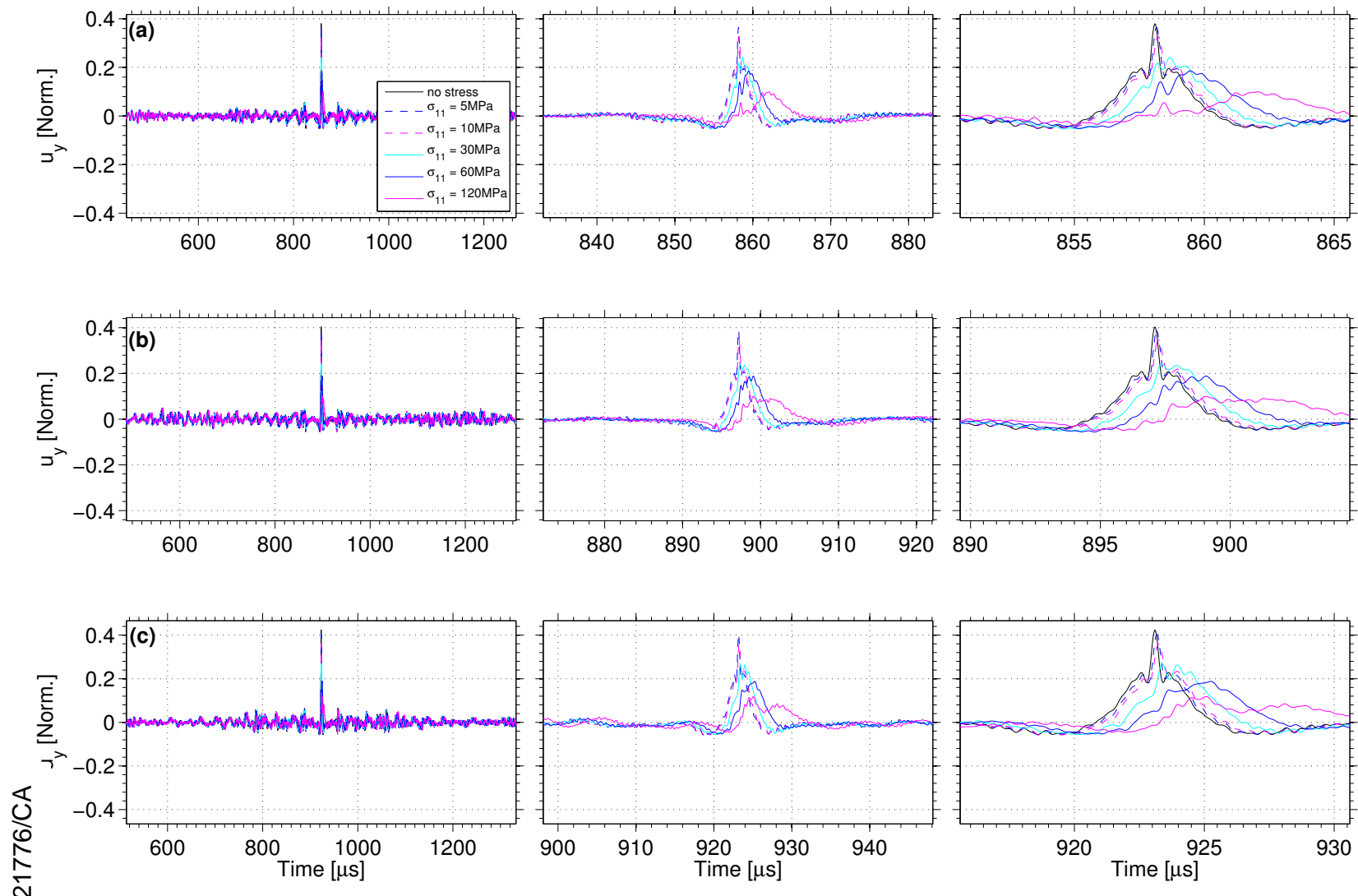

Figure 8.27: Wideband Lamb waves time reversal for several sensed position propagation in finite plate in different zoom levels. (a) $x=210 \mathrm{~mm}$, (b) $x=420 \mathrm{~mm}$ and (c) $x=560 \mathrm{~mm}$

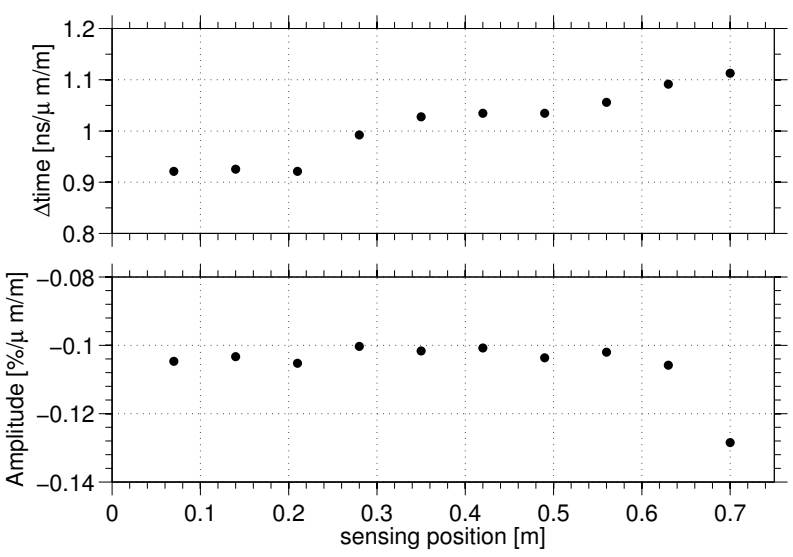

Figure 8.28: Wideband Lamb waves time reversal sensitivity for several sensed position propagation in finite plate

quality of a closed cavity was studied by Pérez et al. [152]. 


\subsubsection{2}

\section{Band limited propagation}

The same filters used for the infinite medium (Table 8.2) were used for the finite length plate. The filtered signals are shown in Fig. 8.29 and the focused signals are shown in Fig. 8.30.
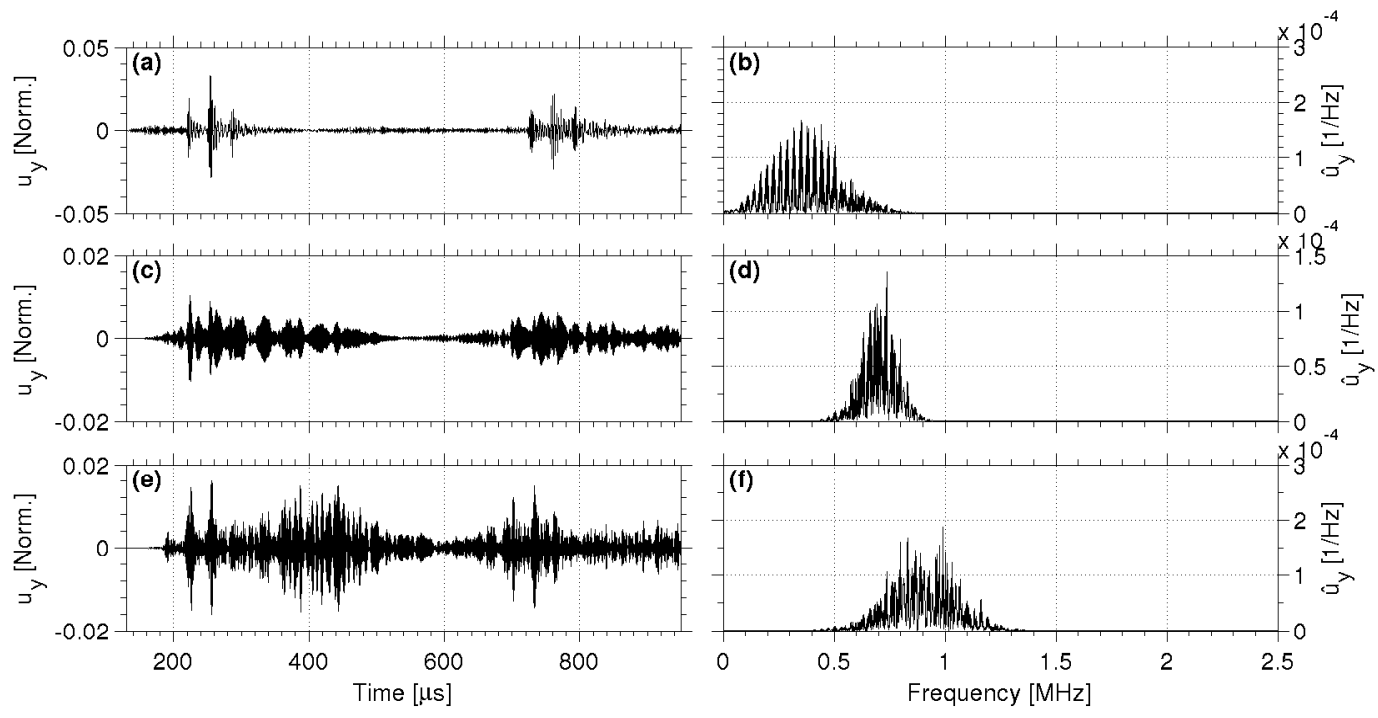

Figure 8.29: Filtered signals for finite plate in time domain (left) and their Fourier transform (right) by filter (a), figures (a) and (b); filter (b), figures (c) and (d); and filter (c), figures (e) and (f).

As for the raw signal the focus center region presents the same shape, but the main peak is significantly higher and there are more energetic side echos.

The peak value and instant against stress are shown in Fig. 8.31. The complex behavior for higher stresses is even more clear. Observing the high stress region in 8.31.b, one can see that the peak decrease is not monotone any more. This is very like the spatial focusing pattern of a acoustic cavity [153-156] or even of Lamb waves in plate [51], which was illustrated in Fig. 7.4. One can then treat this phenomenon as new kind of time reversal focus.

Analogue behavior between this focus and the spatial focus makes sense if one thinks that the stress in the medium induces strain, and then positioning change. This is the geometric effect. However, in addition to this effect, velocity changes, as stated by the acoutoelastic effect, explained in detail in the previous chapters. Thus, this focus does not entirely correspond to the spatial focus, but is rather more complex.

The former cases, in the infinite long plate, do not present this pattern because this new focus widths are very large in those case (they present a low decay rate, see the linear decay rate for low stress in Table 8.3). Thus, 

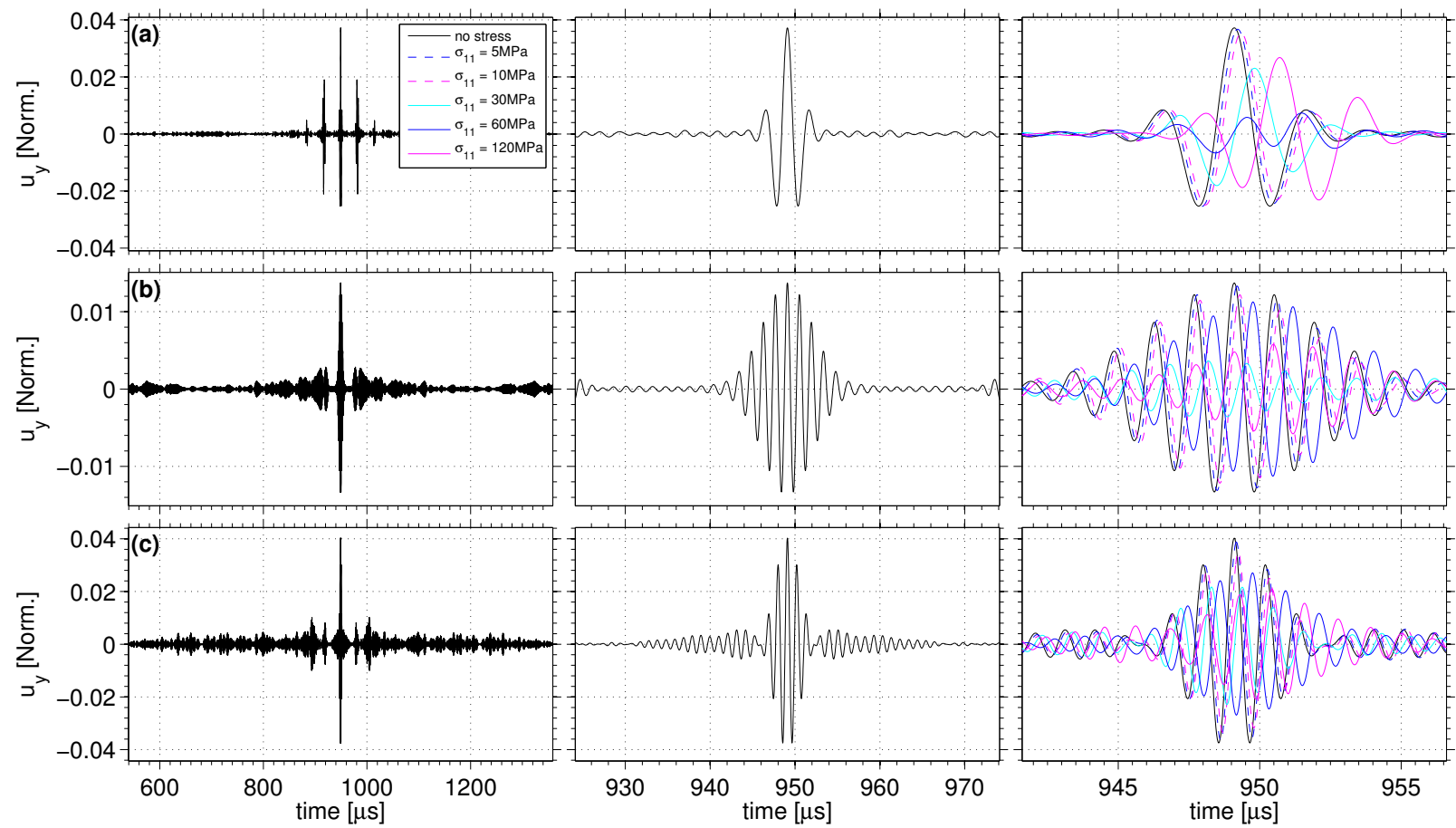

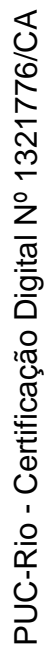

Figure 8.30: Filtered time reversal signals for finite length plate. (a) filter a (b) filter b (c) filter c.

the characteristic pattern does not appear. It is predicted that, if larger stress values were applied one could then visualize the same behavior.

The linear rates for low stress and time focus characteristics are summarized in Table 8.5. The same overall behavior as the one observed for the infinite length plate is present in this finite medium case, the same ordination regarding the filtered signals are found. However, the sensitivities are all higher.

\begin{tabular}{|c|c|c|c|c|c|c|c|}
\hline & \multicolumn{4}{|c|}{ Conventional Time Reversal } & \multicolumn{2}{|c|}{ One-bit time reversal } \\
\hline & & \multicolumn{2}{|c|}{ Focus Quality } & \multicolumn{2}{|c|}{ Low Stress Sens. } & Focus Quality & Low Stress Sens. \\
\hline Filter & Implem. & $\begin{array}{l}\text { Peak val. } \\
\text { (Norm.) }\end{array}$ & $\begin{array}{c}\text { TREF } \\
(\%)\end{array}$ & $\begin{array}{l}\text { Peak decr. } \\
\left(\frac{\%}{\mu m / m}\right)\end{array}$ & $\begin{array}{l}\Delta \mathrm{TOF} \\
\left(\frac{n s}{\mu m / m}\right)\end{array}$ & $\begin{array}{cc}\text { Peak val. } & \text { TREF } \\
\text { (Norm.) } & (\%)\end{array}$ & $\begin{array}{cl}\text { Peak decr. } & \Delta \text { TOF } \\
\left(\frac{\%}{\mu m / m}\right) & \left(\frac{n s}{\mu m / m}\right)\end{array}$ \\
\hline \multirow{2}{*}{ (a) } & Phys. & 0.038 & 20.8 & 0.0314 & 1.76 & ----- & ----- \\
\hline & Corr. & 0.037 & 18.6 & 0.0310 & 1.72 & $0.21 \quad 15.3$ & $0.0339 \quad 1.94$ \\
\hline \multirow{2}{*}{ (b) } & $\overline{\mathrm{P}} \overline{\mathrm{h}} \overline{\mathrm{sis}}$. & $\overline{0} \overline{0} 0 \overline{1} \overline{4}$ & $-\overline{7} . \overline{6} \overline{3}$ & $\overline{0} \overline{0.0 \overline{7}} \overline{2} \overline{3}$ & $\overline{1.4 \overline{2}}$ & & \multirow[b]{2}{*}{$0.0767 \quad 1.52$} \\
\hline & Corr. & 0.014 & 6.94 & 0.0722 & 1.41 & 0.054 & \\
\hline \multirow{2}{*}{ (c) } & $\overline{\text { Phys. }}$ & $\overline{0.0 \overline{4}} \overline{0}$ & $\overline{9} . \overline{6} \overline{2}$ & $\overline{0.0} \overline{8} \overline{4} 3^{-}$ & $1.0 \overline{2}$ & & $---\overline{--}-$ \\
\hline & Corr. & 0.040 & 9.87 & 0.0851 & 1.01 & 0.14 & $0.106 \quad 1.06$ \\
\hline \multirow{2}{*}{ Raw } & $\overline{\mathrm{Ph}} \overline{\mathrm{T}}-\overline{\mathrm{s}}$. & $\overline{0.58^{-}}$ & $4 \overline{6} . \overline{3}-$ & $\overline{0 .} \overline{1} \overline{4} \overline{0}^{-}$ & $1.0 \overline{7}$ & --- & \multirow{2}{*}{$\frac{-1}{0.138}-\frac{-}{1.20}$} \\
\hline & Corr. & 0.56 & 39.1 & 0.128 & 1.11 & 37.8 & \\
\hline
\end{tabular}

Table 8.5: Filtered time reversal focus and sensitivity characteristics, conventional and one-bit, for finite length plate. 


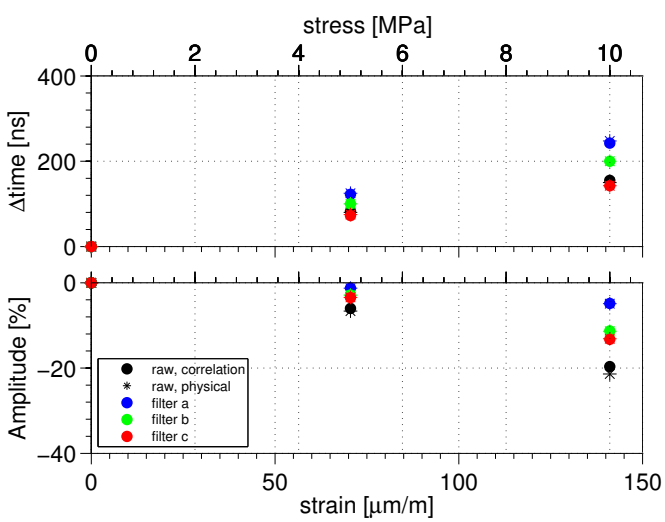

(a)

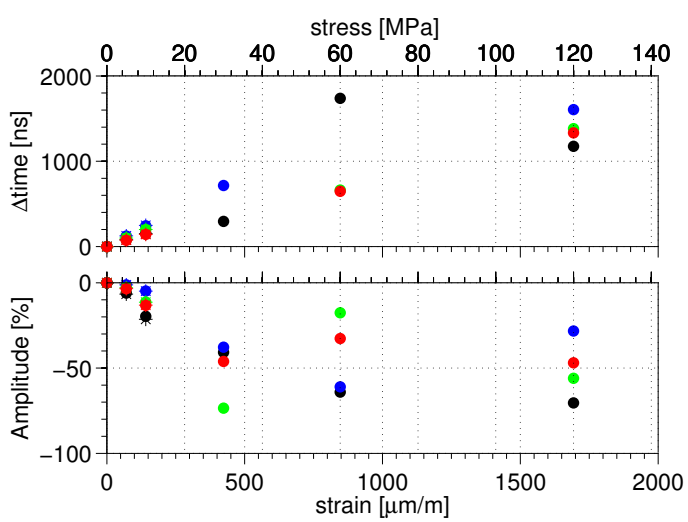

(b)

Figure 8.31: Time reversal focus behavior for filtered signals in finite length plate. Time shift (top) and amplitude relative decrease (bottom). The abscissa is either longitudinal strain, in $\mu \mathrm{m} / \mathrm{m}$ unit, or longitudinal stress, in $M P a$ unit. Black points stand for raw signal. (a) Low stress range. (b) High stress range.

\subsubsection{3}

\section{One-bit time reversal}

The one-bit variation has been tested for the finite length plate as well. The one-bit time reversal signal is shown in Fig. 8.32 for the raw signal and in Fig. 8.33 for the filtered signals.
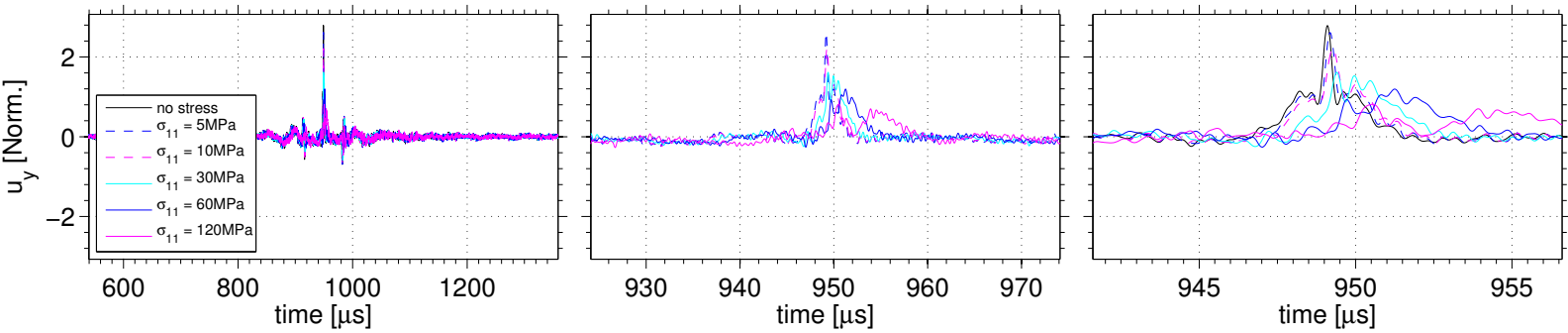

Figure 8.32: Wideband One-Bit Time Reversal signal for finite length plate, in three zoom levels.

The main effects of one-bit implementation are still found in the finite length medium (amplitude gain, loss of symmetry and lower TREF). However, the one-bit time reversal signal is closer to the conventional time reversal in the finite length plate than in the infinite plate (See TREF in Table 8.5). The reason for this behavior, is because the system response is less sparse in the time domain in the finite plate, due to the reflections, and thus there are less intervals where the the maximum excitation, $\pm V$, correlates with region of negligible amplitude; which happens for the infinite medium. This confirm the work of Derode et al. [149] where one-bit time reversal were used in a complex medium (a random set of steel rods) and the focalization properties were shown to be quite similar to those of the traditional time reversal. 

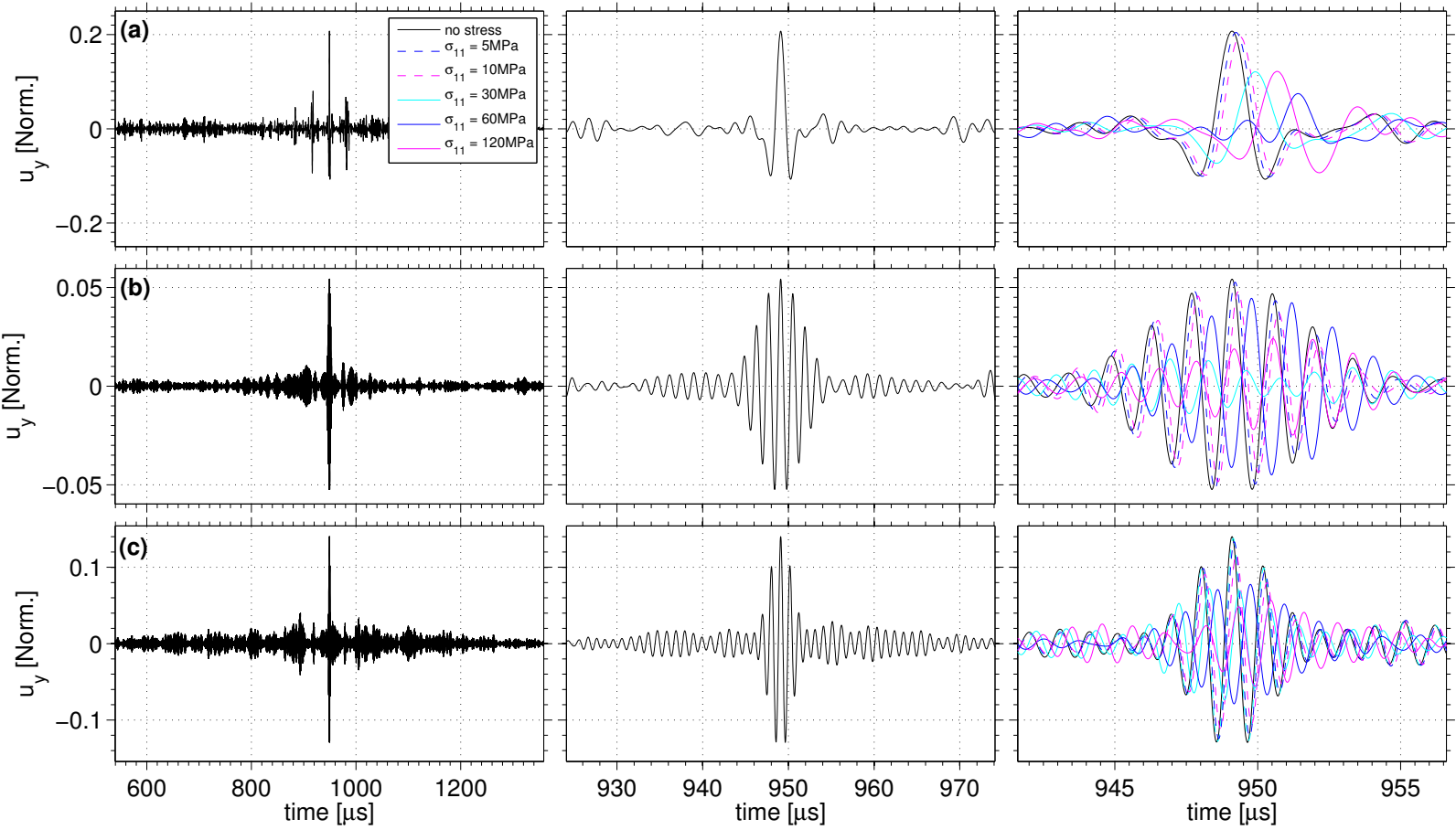

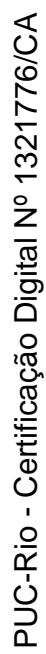

Figure 8.33: Filtered One-Bit Time Reversal signals for finite length plate by filters (a), (b) and (c) respectively.

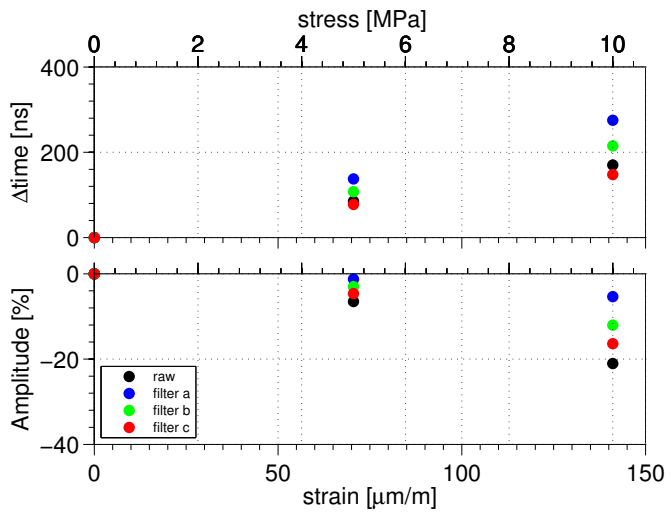

(a)

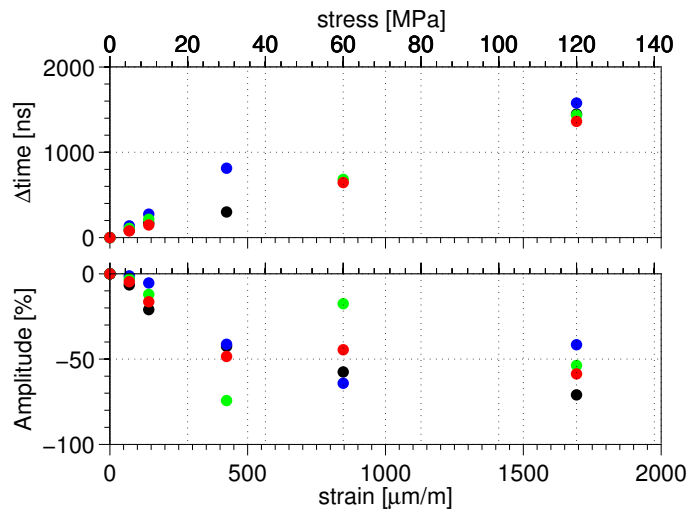

(b)

Figure 8.34: One-bit time reversal focus behavior for filtered signals in finite length plate. Time shift (top) and amplitude relative decrease (bottom). The abscissa is either longitudinal strain, in $\mu \mathrm{m} / \mathrm{m}$ unit, or longitudinal stress, in $M P a$ unit. Black points stand for raw signal.

Regarding the load effects, the peak behavior is shown in Fig. 8.34. The sensitivity for low stress is shown in Table 8.5. There is a consistent slight increase in both peak decrease and time-of-flight delay sensitivity for all cases. The one-bit simplification shows to be feasible for this case, i.e., finite plate under low stress. 


\section{9}

\section{Experiments}

Experiments were performed in an 3-mm thick, 800-mm long and 100$\mathrm{mm}$ wide aluminum plate. The plate is mounted over a bridge structure where a variable traction condition is applied. Fig. 9.1.a sketches the experimental setup, the aluminum plate is mounted over the bridge and fixed at its two ends; at the left end, it is screwed to the main structure (letter F in Fig. 9.1.a) while the right end is fastened to a moving support $(\mathrm{H})$. This support is linked to a threaded rod $(\mathrm{C})$. Tensile stress is imposed onto the plate by threading the hex nut (D). The mounting brackets (E) are used as guidance, at the structure top and bottom, preventing the plate from twisting. As a reference, the actual strain value is measured by a resistive strain-gauge $(G)$ placed in the center of the plate. This setup permits imposing a low value strain range up to 150 $\mu \mathrm{m} / \mathrm{m}$ in the used specimen.

Fig. 9.1.b shows one end of the plate, to which three transducers are glued. The other element of each pair is glued to the opposite extremity of the plate, spaced by $L=700 \mathrm{~mm}$,(points A and B in Fig 4).

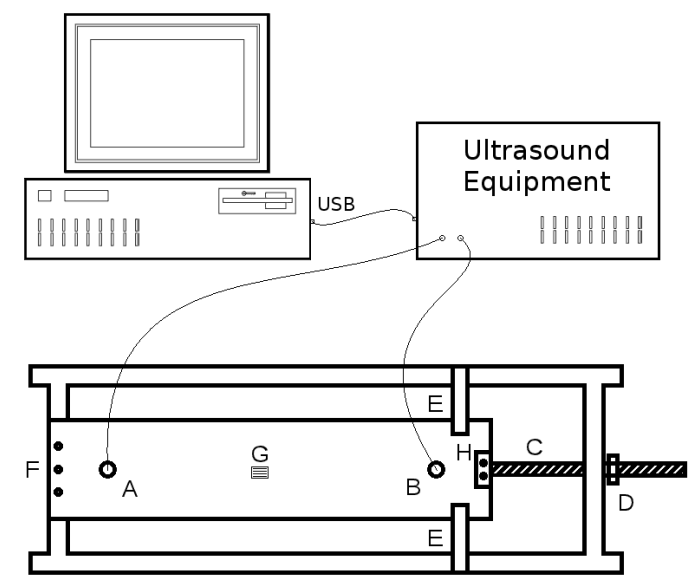

(a)

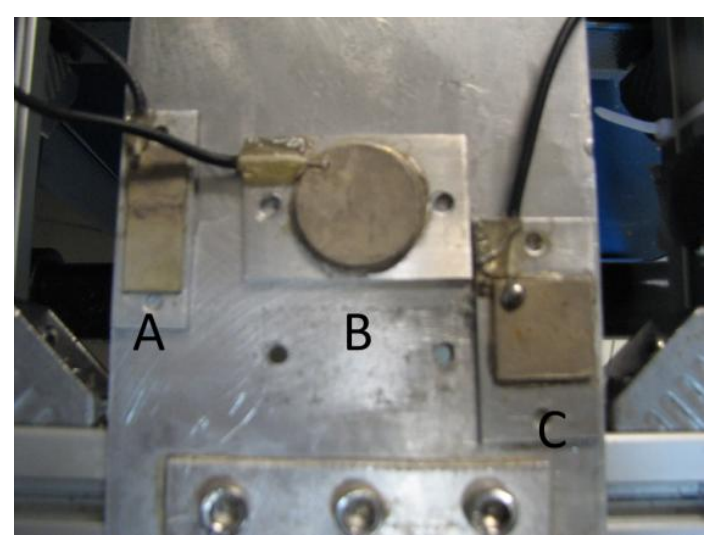

(b)

Figure 9.1: Experimental setup. (a) Tractioning bridge. (b) Transducers bonded at plate.

To generate and to receive arbitrary ultrasonic signals, a commercial ultrasound equipment is used (Open System, LecoeurElectronique, Chuelles, France). This equipment is able to transmit arbitrary waveforms; presents 
a 12-bit analog to digital converter receiver coupled to an ultra-low noise preamplifier with programmable gain up to $80 \mathrm{~dB}$ in a frequency band up to $25 \mathrm{MHz}$ and receiving buffer of 8192 samples [157]. A USB interface allow controlling the equipment from a PC by MATLAB ${ }^{\circledR}[158]$ codes written by the user, as illustrated in Fig. 9.1.a. This ease the bench setup by incorporating the functionalites of an arbitrary waveform generator, power amplifier, preamplifier and oscilloscope, necessary for the experiment.

\section{1}

\section{Piezocomposite transducers}

Three pairs of 2-2 piezocomposite transducers were constructed in order to test the influence of the transfer function on the strain sensitivity. They were made at the Ultrasound Laboratory of Polytechnic School of the University of São Paulo (EPUSP). The fabrication processes is the standard dice and fill procedure, commonly used in piezocomposite manufacturing. The piezoelectric ceramic chosen is PZ37 from Ferroperm [159]. This material was developed for NDE applications. It has very low acoustic impedance and a high thickness coupling coefficient. 2-2 piezocomposite transducers are constructed by cutting the ceramics in one direction, making parallel bars and filling the space between the bars with polymer [160]. This transducer type has lower impedance and a wider frequency band than single ceramic transducers. To make the final assemblage more robust and easy to handle, each sample is glued into a small aluminum base as shown in Fig. 9.1.b. Table 9.1 summarizes the constructive characteristics of the transducers.

\begin{tabular}{c|cccc}
\hline $\begin{array}{c}\text { Nominal } \\
\text { Frequency }\end{array}$ & $\begin{array}{c}\text { Ceramic } \\
\text { thickness }(\mathrm{mm})\end{array}$ & $\begin{array}{c}\text { Element } \\
\text { width }(\mathrm{mm})\end{array}$ & $\begin{array}{c}\text { Polymer } \\
\text { width }(\mathrm{mm})\end{array}$ & $\begin{array}{c}\text { Pitch } \\
(\mathrm{mm})\end{array}$ \\
\hline $500 \mathrm{kHz}$ & 1.75 & 1.0 & 0.2 & 1.2 \\
$1 \mathrm{MHz}$ & 1.43 & 0.2 & 0.06 & 0.26 \\
$2.25 \mathrm{MHz}$ & 0.65 & 0.1 & 0.04 & 0.14
\end{tabular}

Table 9.1: Piezocomposites constructive characteristics.

In a piezocomposite material, the final resonant frequency is lower than the one corresponding to a single ceramic of the same thickness. This is explained because the material presents intermediate properties between the ceramic and the polymer. As the propagation speed in the polymer is lower than that of the ceramic, the final frequency in the piezocomposites is lower than in the original ceramics used for their construction, depending on the volume fraction of ceramic in the composite [161]. The most important fact in the use of composite is a higher bandwidth to excite the different modes in the plate. The transfer functions are exhibited in Fig. 9.2, where the frequency 
content of the systems is visible. The frequencies of the ceramics are still used to tag the piezocomposites in the remainder of the text.

During the loading process practical issues arose related to the transducers bonding on the plate. It has been observed that the signal received was varying considerably between repetitions of the same experiments. After some time spent investigating this issue, it was discovered that the transducers were not properly bonded on the surface. The loading and unloading process made them to progressively become loose. The bonding process needs to be done properly to resist to the loading.

To overcome this, a better bonding process was achieved by proper cleaning and sanding the specimen surface before bonding the transducers. Then, an overnight curing time under pressure was adopted. After that the transducers seemed to be tighten to the surface. Some repetitions were performed and the results showed to be coherent, confirming the quality of adhesiveness. The former experimental results were disregarded and a complete new set of experiments were acquired.

\section{2 \\ Time reversal in plates}

Initially, a sinc pulse was used to excite the transmitting element, Te. This pulse is the same used in the simulations, see Fig. 8.1, presenting a flat bandwidth in the working frequency range, up to $2.5 \mathrm{MHz}$. Then the signal received at the opposite element $\operatorname{Tr}$ is equivalent to the impulse response, and its Fourier transform is the system transfer function. The observable time window is the same used in simulation, i.e., $\Delta T=819.1 \mu \mathrm{s}$, from $t_{0}=130.0 \mu \mathrm{s}$, when the wave front from the fastest mode arrive, up to $t_{0}+\Delta T=949.1 \mu \mathrm{s}$.

Fig. 9.2 shows the signal received using different transducers for null strain condition, corresponding to the impulse response and the transfer function for each pair.

The signals at zero strain state, shown in Fig. 9.2, are then time-reversed and used as a reference to be retransmitted from $T e$. A new signal $y_{1}(t)$ is received at $\operatorname{Tr}$ as a response to the reversed reference signal, as calculated in (7.11) or (7.9). The resulting focused signals for null strain can be observed in Fig. 9.3.a, c and d; a zoom on the focus region are shown in Fig. 9.3.b, d and e, respectively. Time reversal characteristics can be observed, such as time compression, high focus amplitude and signal symmetry.

Fig. 9.3 allows us to observe the different focus quality achieved with each transducer pair. The TREF values of each setup are summarized in Table 9.2 , as well as the signal-to-noise ratio (SNR). The following definition was 

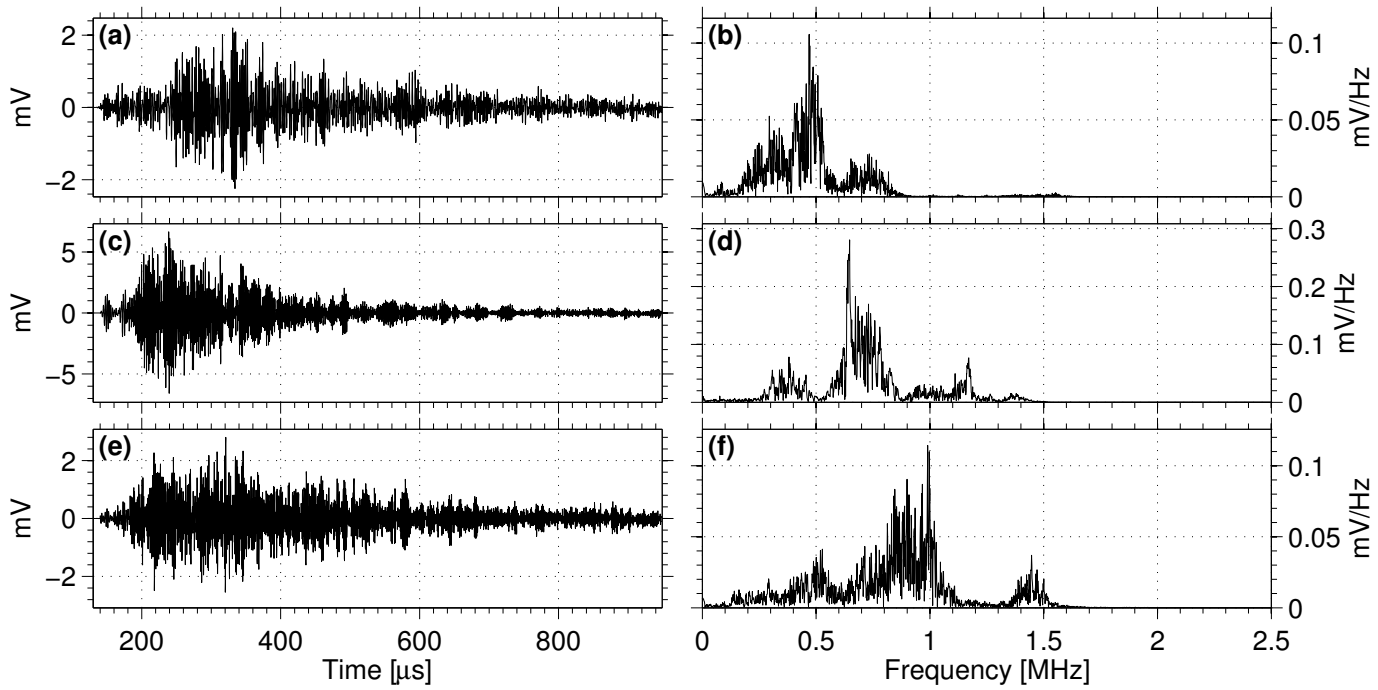

Figure 9.2: Received forward signals and their Fourier spectra for $0.5 \mathrm{MHz}$, (a) and (b); $1 \mathrm{MHz},(\mathrm{c})$ and (d); and $2.25 \mathrm{MHz},(\mathrm{e})$ and (f), transducers
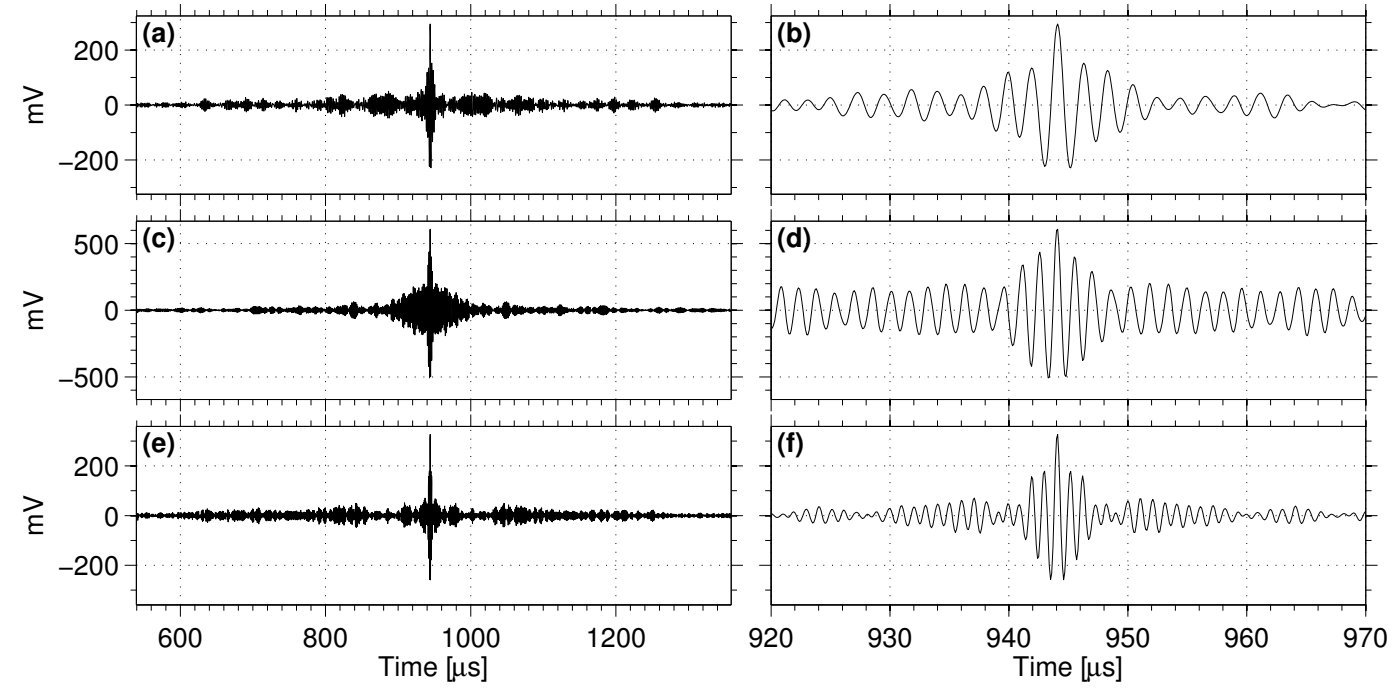

Figure 9.3: Received time reversal signal. The $0.5 \mathrm{MHz}, 1 \mathrm{MHz}$ and $2.25 \mathrm{MHz}$ transducer responses are shown in (a), (c) and (e), respectively. Zooms on focused time are shown in (b), (d) and (f), for their respective transducer pairs.

adopted

$$
S N R=10 \log _{10}\left(\frac{E_{s}}{E_{n}}\right),
$$

where $S N R$ is expressed in $d B, E_{n}$ is the mean noise energy of the equipment and transducer, and $E_{s}$ is the energy of the signal. It is considered here the energy of the peak as for the signal, hence $E_{s}=E_{p k}$ as defined in (7.13b). 


\subsection{1}

\section{Strain sensing}

To evaluate the changes in the time reversal process, a set of signals are acquired at $\operatorname{Tr}$ for the whole traction range, always transmitting the original time-reversed reference signal by $T e$. The tensile load is applied in steps from zero up to about $150 \mu \mathrm{m} / \mathrm{m}$. For each traction level, a time reversal response is acquired and recorded.

When traction is applied, the time reversal signal is altered due to the changes imposed on the plate. The behavior of the focus amplitude and the focalization time-shift, as a function of the principal strain value, are shown in Fig. 9.4 for the whole experiment of each transducer pair. The experiment is performed by increasing and decreasing the mechanical load, recovering the initial condition at the end of the cycle. Fig. 9.4 allows observing that both time-of-flight and amplitude were recovered when the plate returns to the initial zero strain level. This behavior indicates that for the experiment duration, the strain dependence is more relevant than any other varying condition, such as temperature variation.

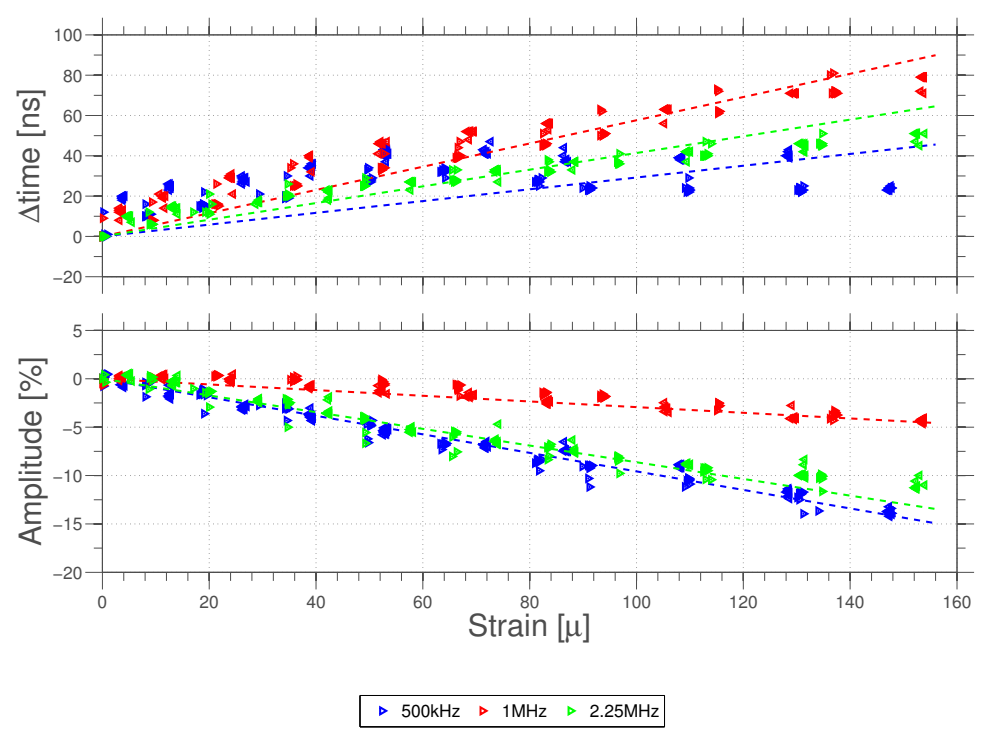

Figure 9.4: Time reversal strain sensitivity. Peak reduction (bottom) and time of flight shift (top) for conventional time reversal for the whole traction range applied. The right arrow indicates the load is going up and the left one that the load is going down. The dashed lines represent the linear fit for each setup.

For each experiment, a linear coefficient for the amplitude reduction and focalization time delay is calculated. These values are also reported in Table 9.2. The SNR and TREF for null strain state are presented in the 
first and second columns, respectively; in the peak column, the estimated (mean) sensitivity for the amplitude decreases and its standard deviation (std.) in percent per imposed strain; in the $\triangle \mathrm{TOF}$ column, the estimated (mean) sensitivity for the time-of-flight delay and its standard deviation (std.), in $n s$ per imposed strain.

The experimental results were also analyzed for linearity assessment. The Coefficient of Determination $\left(R^{2}\right)$ for the linear fit of the experimental data was calculated and reported in Table 9.2, in the $R^{2}$ column. The Coefficient of Determination is defined in (9.2) and is frequently used to judge the model goodness $[162,163]$,

$$
R^{2}=1-\frac{\sum_{i}\left(D_{i}-M_{i}\right)^{2}}{\sum_{i} D_{i}^{2}}
$$

where D represents the data; here, either the amplitude decrease or the timeof-flight shift, and $\mathrm{M}$, the fitted values by the linear model. $R^{2}$ varies from 0 to 1 ; the better the model fits the data, the closer the value of $R^{2}$ is to 1 . The definition in (9.2) is more suited for a fit trough origin [162], which has been used here, since there is no physical reason to present a non-zero constant term.

\begin{tabular}{ccc|cccccc}
\hline \multirow{2}{*}{ Experiments } & SNR & TREF & \multicolumn{3}{|c}{ Peak decrease } & \multicolumn{3}{c}{$\Delta$ TOF } \\
& $(d B)$ & $(\%)$ & \multicolumn{2}{c}{$\left(\frac{\%}{\mu m / m}\right)$} & & $\left(\frac{n s}{\mu m / m}\right)$ & $R^{2}$ \\
\hline $0.5 \mathrm{MHz}$ & 64 & 14.7 & 0.096 & 0.00055 & 0.980 & 0.29 & 0.0076 & 0.701 \\
$1 \mathrm{MHz}$ & 71 & 8.73 & 0.029 & 0.00024 & 0.963 & 0.58 & 0.0044 & 0.969 \\
$2 \mathrm{MHz}$ & 66 & 12.3 & 0.086 & 0.00060 & 0.976 & 0.41 & 0.0038 & 0.960 \\
$0.5 \mathrm{MHz} \& 2 \mathrm{MHz}$ & 66 & 23.3 & 0.103 & 0.00054 & 0.982 & 0.33 & 0.0032 & 0.942
\end{tabular}

Table 9.2: Sensitivity Results.

Regarding linearity and goodness of fit, it is clear that no linearity is present in the time delay observation for the $0.5 \mathrm{MHz}$ experience, whose $R^{2}$ value are about $70 \%$, indicating that it does not behave linearly with strain. This case was the only experimental measure that presented a value below $90 \%$. Lower linearity for low frequency at low strain has been reported elsewhere [164], where it was hypothesized that specimen fixation accommodation due to stress may influence the echoes; this effect is also possible in the present setup as reflected echoes from the plate end, where it is fixed to the bridge, are free to occur and can be detected by the receiver transducer. This measure presented the greatest uncertainty, highest standard deviation; all the others have standard deviation of about $1 \%$ of the estimated value. The remainder $\Delta \mathrm{TOF}$ are more linear, but tend to present a lower $R^{2}$ than the corresponding peak decrease for the same experiment. The peak observation showed to be 
quite linear, especially for the $0.5 \mathrm{MHz}$ and $2.25 \mathrm{MHz}$ transducers experiment, in which TREF was higher.

Note that the resolution in time-shift presented in Fig. 9.4 and Table 9.2 is better than the interval introduced by the period of the $10 \mathrm{MHz}$ sampling frequency. These more precise values were obtained by performing a low-pass interpolation in the raw data by a factor of 100 , preceded by a low-pass filtering. Low-pass interpolation is possible due to the band limited spectrum in the transfer function, see Fig. 9.2. Thus, the time resolution is equivalent to a sampling rate of $1 \mathrm{GHz}$ and a time resolution of $1 \mathrm{~ns}$ is reached.

\section{2 .2}

\section{Transducer combination}

Aiming to enhance the received signal spectrum content, and its amplitude sensitivity, a combination of transducers was used. When a $1 \mathrm{MHz}$ transducer pair was used simultaneously with any other pair, or even all three together, no significant improvement was achieved. This is because the $1 \mathrm{MHz}$ transducer has higher impulse response gain, about twice as high as the others. In this way, the spectrum signature of the latter predominates and no benefit of the combination arises. However, the $0.5 \mathrm{MHz}$ and $2.25 \mathrm{MHz}$ transducer pairs present similar amplitude in the transfer function. Thus, when used together, they produce a richer spectrum. Fig. 9.5 shows its impulse response, both in time and in frequency domain. One can clearly see that the two main spectrum regions have almost the same intensity. As the amplitude gains of both are about the same, there is not any response standing out over the other and both equally collaborate to the impulse response.
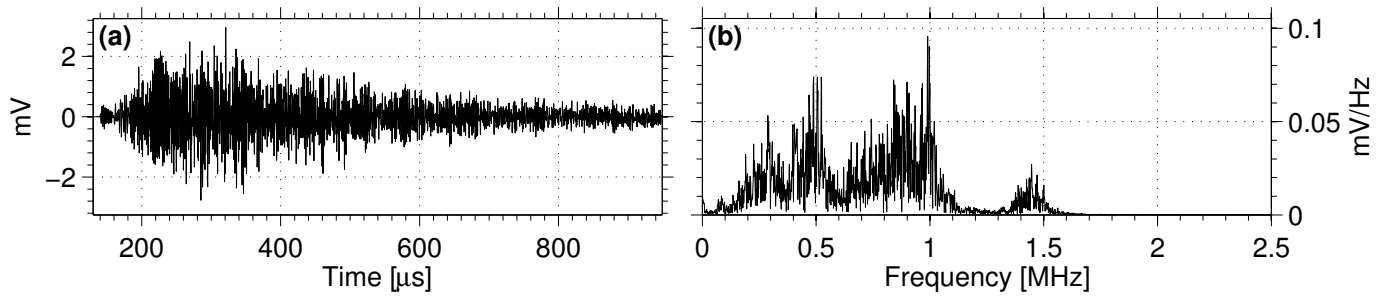

Figure 9.5: Received forward signals (a) and their Fourier spectrum (b) for combination of $0.5 \mathrm{MHz}$ and $2.25 \mathrm{MHz}$

The time reversal signal is shown in Fig. 9.6. Is is clear the sharpest focus peak when compared to the former experiments; i.e., higher time reversal efficiency than all others experiments. The TREF values and the sensitivity are shown in the last row of Table 9.2. This configuration presented the highest amplitude sensitivity, about $0.1 \% / \mu \varepsilon$. 

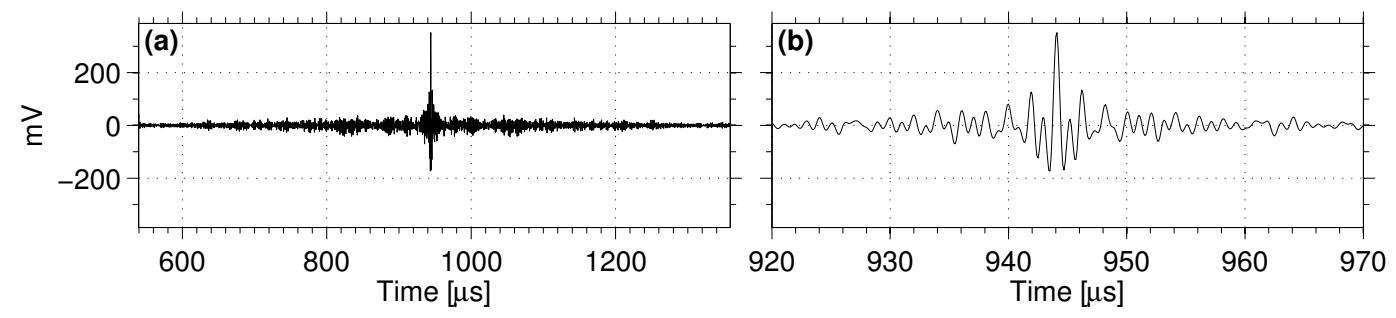

Figure 9.6: Received time reversal signal for combination of $0.5 \mathrm{MHz}$ and $2.25 \mathrm{MHz}$ transducers, response are shown in (a), zoom on focused time are shown in (b).

\section{3}

\section{Strain sensing by one-bit time reversal in plates}

The same procedure was used for the one-bit time reversal as explained in section 7.5. Fig. 9.7 presents the focused signals obtained using one-bit time reversal for each transducer pair. Comparing to Fig. 9.3, one can observe an amplitude gain of 3.5 times (increasing the SNR in about $11 \mathrm{~dB}$, see Table 9.3) compared to the conventional time reversal experiments and loss of symmetry, as expected. However, the focusing capability is verified to be maintained in the neighborhood of the focus; see the zoomed signals in Fig. 9.7. The energy efficiency indicates how the one-bit procedure degenerates the focus. As expected, the TREF values calculated for the conventional time reversal are always greater than the corresponding value for the one-bit time reversal version. For example, in the $0.5 \mathrm{MHz}$ setup, the TREF value for conventional time reversal is $14.7 \%$, whereas for the one-bit variation, it decreases to $13.7 \%$. Table 9.3 summarizes the TREF.

For the one-bit procedure, the plate structure is subjected to longitudinal strain. The sensitivity is observed in the time reversal signal by means of the peak amplitude decrease and the delay in the focalization time, exactly in the same way performed for the conventional procedure. The trend for the amplitude and time-of-flight versus strain is quite similar to the conventional technique as observed in Fig. 9.8. The sensitivities are also presented in Table 9.3 .

Despite the lower TREF, the amplitude sensitivities revealed to be slightly higher than their normal time reversal version, at least 10\% depending on the transducer, and the one-bit time reversal showed to be a suitable improvement for strain monitoring. This is possibly explained by the fact that on the one-bit variation, the time reversal must match the sign of the reference signal, highlighting phase differences. Hence, a phase-shift has a more severe effect on the correlation integration, see (7.23), causing the overall mismatch to be higher. The $R^{2}$ value for the one-bit version is a little lower than its 

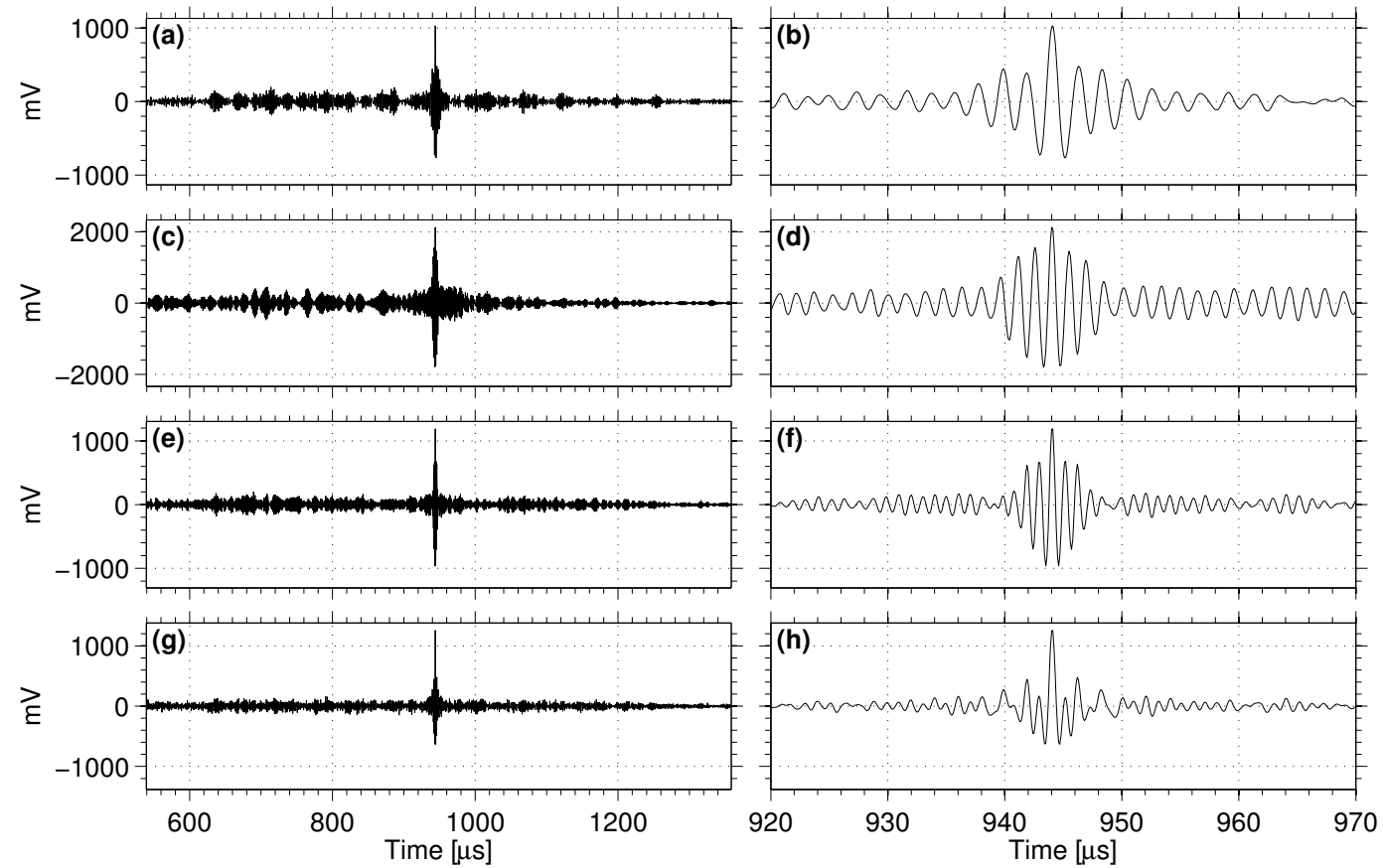

Figure 9.7: Received one-bit time reversal signal. The $0.5 \mathrm{MHz}, 1 \mathrm{MHz}$ and $2.25 \mathrm{MHz}$ transducer and $0.5 \mathrm{MHz}$ and $2.25 \mathrm{MHz}$ combination responses are shown in (a), (c), (e) and (g) respectively. Zooms on focused time are shown in (b), (d), (f) and (g), for their respective transducer pairs.

counterpart in the conventional time reversal, also probably due to more severe phase effect on the signals focus highlighted by the one-bit excitation.

\begin{tabular}{ccc|cccccc}
\hline \multirow{2}{*}{ Experiments } & SNR & TREF & \multicolumn{3}{|c}{ Peak decrease } & \multicolumn{3}{c}{$\Delta$ TOF } \\
& $(d B)$ & $(\%)$ & \multicolumn{2}{c}{$\left(\frac{\%}{\mu m / m}\right)$} & & $\left(\frac{n s}{\mu m / m}\right)$ & $R^{2}$ \\
\hline $0.5 \mathrm{MHz}$ & 75 & 13.7 & 0.121 & 0.00085 & 0.971 & 0.35 & 0.0088 & 0.701 \\
$1 \mathrm{MHz}$ & 82 & 7.97 & 0.063 & 0.00048 & 0.969 & 0.58 & 0.0059 & 0.945 \\
$2 \mathrm{MHz}$ & 77 & 11.4 & 0.094 & 0.00079 & 0.966 & 0.44 & 0.0046 & 0.951 \\
$0.5 \mathrm{MHz} \& 2 \mathrm{MHz}$ & 76 & 22.4 & 0.127 & 0.00074 & 0.978 & 0.36 & 0.0039 & 0.931
\end{tabular}

Table 9.3: Sensitivity Results for One-bit time reversal. 


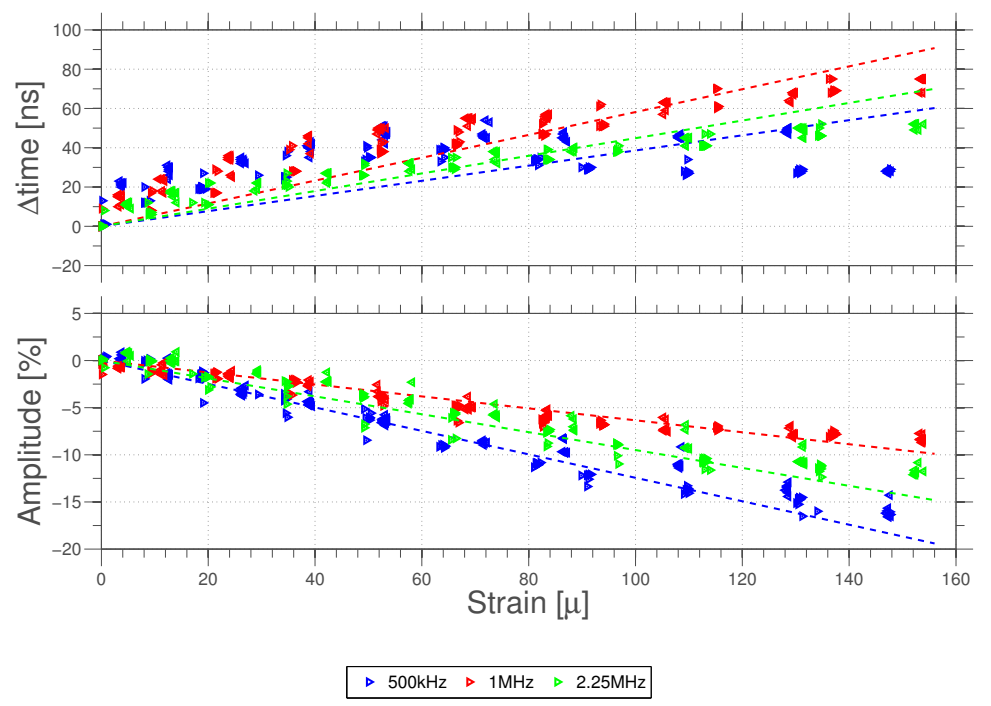

Figure 9.8: One-bit Time reversal strain sensitivity. Peak reduction (bottom) and time of flight shift (top) for one-bit time reversal experiment. The right arrow indicates the load is going up and the left one that the load is going down. The dashed lines represent the linear fit for each setup

\section{4}

Strain sensing by correlation implementation of time reversal in plates

As indicated in section 7 , the time reversal process is a physical implementation for the cross-correlation operation. For the case of a single emitter and receiver, it is straightforward to compute a numerical equivalent for the time reversal process, i.e., there is no need to perform an analogical emission to obtain the equivalent time reversed signal. The impulse response for the null strain and the impulse response at each strain state can be acquired and then these two signals are used to perform the cross correlation as exposed in section 7 . In practice, the main difference is between the cost and complexity of the evolved electronics. In the traditional time reversal, an analog emitter is needed. But in the numerical post-processing, more computational resources are used for cross correlation implementation, implying more time and power consumption. Also, the numerical implementation does not enjoy the benefit of high SNR in the focused signal intrinsically obtained by the physical implementation.

The numerical implementation was experimentally investigated. The focused signal for either conventional and one-bit time reversal are shown in Fig. 9.9 and Fig. 9.10, respectively. As for the simulations, the correlation and physical implementation showed to be equivalent also for the real experiments. 

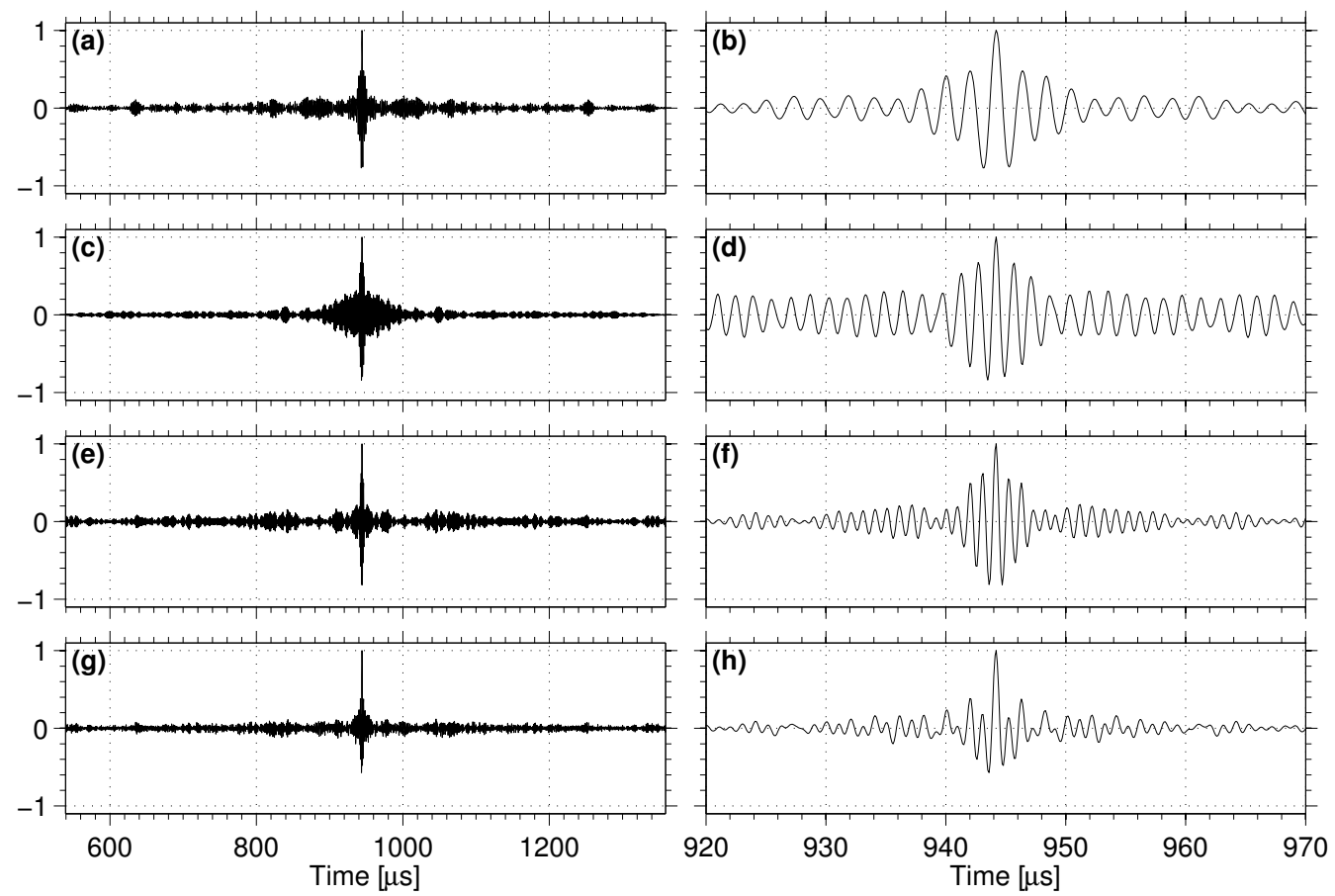

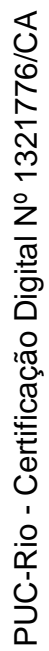

Figure 9.9: Correlation implementation of time reversal signal. The $0.5 \mathrm{MHz}$, $1 \mathrm{MHz}$ and $2.25 \mathrm{MHz}$ transducer and $0.5 \mathrm{MHz}$ and $2.25 \mathrm{MHz}$ combination responses are shown in (a), (c), (e) and (g) respectively. Zooms on focused time are shown in (b), (d), (f) and (g), for their respective transducer pairs.

The sensitivity results are almost the same for the physical time reversal and for the correlation implementation. Table 9.4 summarizes the results of the numerical implementation. For the sensing procedure presented here, it was confirmed that the choice is between the use of an analog hardware in the emission to reverse and to retransmit the signal or more processing power to post-process the signals. Also regarding the sensitivity uncertainty and the deviation from linearity, the same overall conclusions could be drawn, guaranteeing no additional issues for the numerical usage.

\begin{tabular}{|c|c|c|c|c|c|c|c|c|c|c|c|c|c|}
\hline \multirow[b]{3}{*}{$\begin{array}{l}\text { Exp. } \\
(M H z)\end{array}$} & \multicolumn{6}{|c|}{ Conventional Time Reversal } & \multicolumn{7}{|c|}{ One-Bit Time Reversal } \\
\hline & & Peak decrea & & & $\Delta \mathrm{TOF}$ & & & & ak decre & & & & \\
\hline & $\begin{array}{c}\text { TREF } \\
(\%)\end{array}$ & $\begin{array}{l}\text { mean } \\
\left(\frac{\%}{\mu m / m}\right)\end{array}$ & $R^{2}$ & $\begin{array}{l}\text { mean } \\
\qquad\left(\frac{\imath}{\mu n}\right)\end{array}$ & $\begin{array}{l}\text { std. } \\
\left.\frac{s}{m}\right)\end{array}$ & $R^{2}$ & $\begin{array}{c}\text { TREF } \\
(\%)\end{array}$ & mean & $\frac{\text { std. }}{\left(\frac{6}{m}\right)}$ & $R^{2}$ & mean & std. & $R^{2}$ \\
\hline 0.5 & 15.2 & $0.094 \quad 0.00027$ & 0.995 & 0.28 & 0.0070 & & 13.6 & 0.118 & 0.00057 & 0.98 & 0.31 & 0.0081 & 0.700 \\
\hline 1 & 8.62 & 0.00022 & 0.980 & 0.59 & 0.0042 & 9.971 & 5.68 & 0.074 & 0.00054 & 0.970 & 0.59 & 0.0058 & 0.950 \\
\hline 2.25 & 11.1 & 0.00030 & 0.994 & 0.44 & 0.0038 & 0.964 & 9.64 & 0.113 & 0.00047 & 0.992 & 0.47 & 0.0046 & 0.955 \\
\hline $0.5 \& 2.25$ & 20.6 & 0.00036 & 0.992 & 0.35 & 0.0032 & 0.951 & 17.6 & 0.135 & 0.00051 & 0.991 & 0.37 & 0.0037 & 0.940 \\
\hline
\end{tabular}

Table 9.4: Sensitivity results for correlation implementation. 

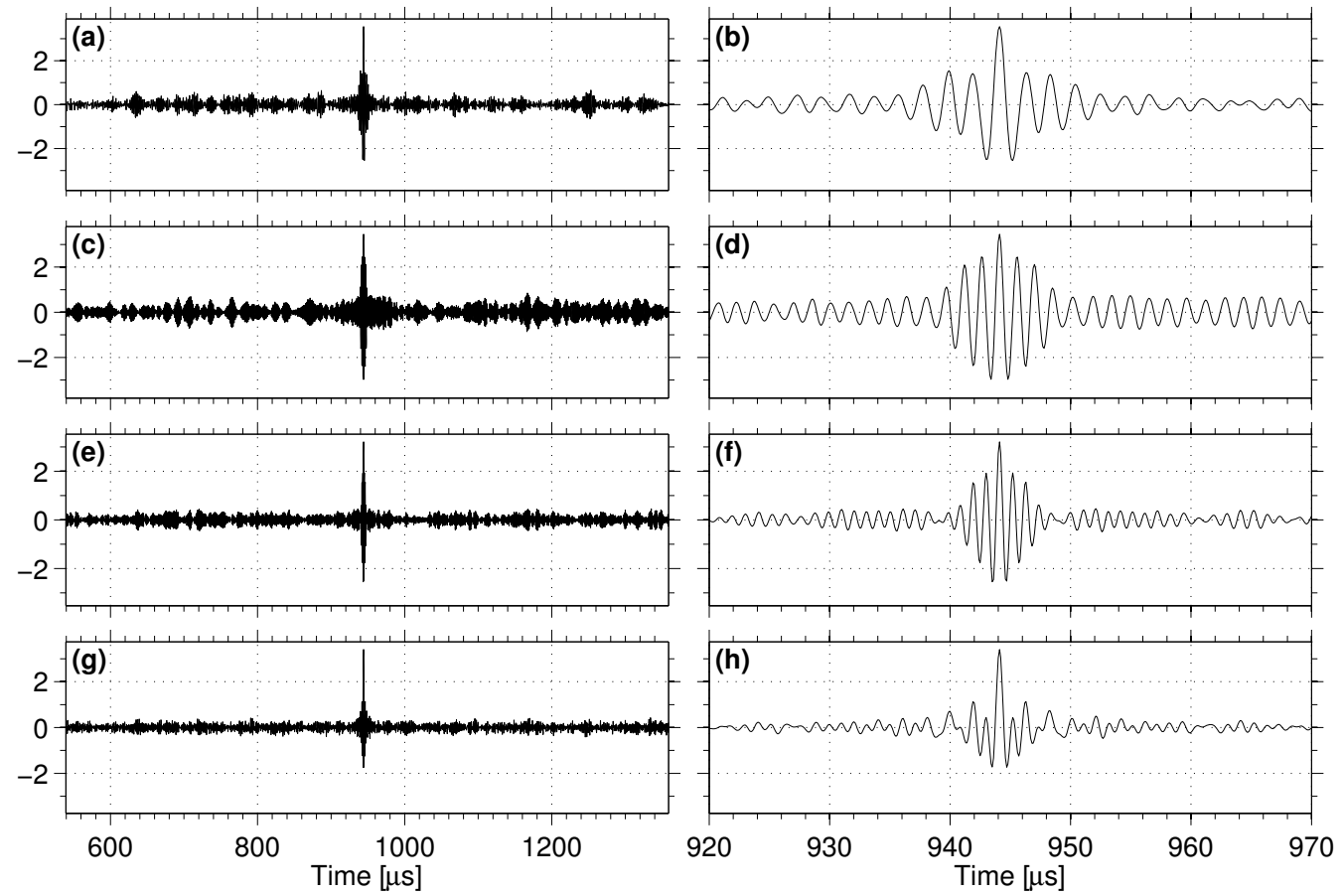

Figure 9.10: Correlation implementation of one-bit time reversal signal. The $0.5 \mathrm{MHz}, 1 \mathrm{MHz}$ and $2.25 \mathrm{MHz}$ transducer and $0.5 \mathrm{MHz}$ and $2.25 \mathrm{MHz}$ combination responses are shown in (a), (c), (e) and (g) respectively. Respective zooms on focused time are shown in (b), (d), (f) and (g).

\section{5}

\section{Comparison between experiments and simulation}

In this section the experimental signals and results are compared to the FEM simulation in Chapter 8, specially for the finite length plate, in section 8.2. The filters designed in that chapter, (a), (b) and (c) (see Fig. 8.29), have about the same spectral content of the spectrum main regions of the experimental signals, transducers $0.5 \mathrm{MHz}, 1 \mathrm{MHz}$ and $2.25 \mathrm{MHz}$, respectively (see Fig. 9.2). However, the signals obtained in the simulation are far from the experimental ones. There are several reasons for this.

The most evident is that the reflected echoes in the real signals are not so intense as in the simulation. This happens due to damping, that makes the more distant echoes to attenuate; also energy escapes from the plate to the mounting structure, and, in minor effect, to the surrounding atmosphere. This implies that reflected waves are less intense than the direct ones.

Other factor is the nature of reflections near the end. In the real system there may be several reflections in each ending, due to the mounting of the plate on the structure by screws and nuts. The presence of the transducers, bonded on the plate, reflect waves as well. Thus the reflection at the real plate should be far more complex than the flat end that has been modeled in the 
simulation.

The finite width of the plate leads to a difference between simulation and real experiment, which is an additional possible source of discrepancy. In the experimental setup wave fronts can be reflected by the finite width. Thus there are two guidance principles, by the thickness, that imply in the Lamb modes, and by the width.

The vibration principle of the transducers is different. In the simulations, a single out-of-plane excitations has been applied in just one point. In the experiment, the piezoelectric actuator has finite dimensions; it may introduces strain into the medium in all the directions and orientations depending on its electro-mechanical constitutive equations and geometry $[15,165,166]$. The same is valid for the receiver, i.e., the electric signal that is sensed is the result of the mechano-electrical coupling, and not of a simple out-of-plane displacement as used in the simulations. Thus, not only the excitation but also the filtering used in the simulation are rough approximations for the effect of the transducer on actuating and reading the waves. Nevertheless, they do illustrate the effect of wider/narrower bandwidth in the propagation of the Lamb waves. Even so, the filtered signal bands do not present the spectral content outside their main lobe. As for the real signals, it is observable meaningful amplitude on Fourier transform in regions outside the center lobe, as at $700 k \mathrm{~Hz}$ for Fig. 9.2.b; $400 \mathrm{kHz}$ and $1200 \mathrm{kHz}$ for Fig. 9.2.d and $500 \mathrm{kHz}$ and $1500 \mathrm{kHz}$ for Fig. 9.2.f. These are not presented in the simulated filtered signals.

The elastic constants used in simulations, either second and third order ones, were taken from tabulated values and not measured of the specimen sample. As it was said before, there is a great divergence in the values of third order elastic constants. Thus a precision comparison, specially in the time-offlight shift, is not possible without the exact set of constants.

Despite these differences some general results can be compared. The peak sensitivity for the experiments (Table 9.2) and finite length simulation (Table 8.5) show values about the same order. Additionally, if one considers the transducer combination as possibly corresponding to the raw signal in experiments, these values also show about the same order.

The time of flight shift, on the other hand, shows much higher sensitivity in the simulation than experiments and seems to be more consistent with the infinite length plate (see Table 8.3). This suggests that in the experiments the reflection at the plate end are not so intense as in the ideal finite length plate that has been simulated. The energy that escapes to the mounting structure and due to damping is relevant.

Other source of difference is the fact that the simulations were carried 
out by EEC approximation. By the sensitivity error curves in Fig. 5.21 it is clear that EEC sensitivities are more negative than the exact ones. That is, the phase velocity is more sensitivity, in absolute value, to stress in the EEC approximation. This can lead to more sensitive peak shift in the simulations and may not necessary influence the peak decrease. This is due to the fact that for the peak to decrease, what is relevant is the different sensitivities on each component of the signal. This is exactly as noticed in section 8.1.1.1 where the analytical model for the time reversal obtained by EEC approximation was compared to the one obtained by means of the exact dispersion curves.

One important characteristics is that in both, finite length simulation and experiments, the peak decrease and time-of-flight shift behave on the opposite direction. That is, where peak decrease is more accentuated, the time shift is less preeminent.

Comparing the one-bit implementation the same overall trend is present in both the simulation and experiment. That is, amplitude gain and a slightly higher sensitivity for peak decrease and shift. This happens for all the transducer pair, or filters in the case of simulation.

A relevant difference from experiment and simulation is the high peak decrease for the $0.5 \mathrm{MHz}$ transducer pair, which is not reproduced by simulation. This is the only case where the sensitivity ordination is different between simulations and experiments. One can speculate that for the low frequency the predominant $A 0$ mode present huge error when addressed by the EEC (see Fig.5.19), where the non-zero velocity for DC component is not reproducible by EEC. The considerable difference occurs for frequencies up to about $200 \mathrm{kHz}$ in a $3 \mathrm{~mm}$ thick plate (equivalent to $600 \mathrm{kHz} \times \mathrm{mm}$ where in Fig. 5.19.b and 5.21.a the errors predominate). Thus, this effect on the simulated signal filtered by (a) (see signals spectrum in Fig.8.29) or, equivalently, by the 0.5 MHz experiment (see Fig.9.2.a), can be relevant.

The experimental results exhibit a clear trend. The higher the TREF, the higher is the amplitude decrease. This corroborates the assumption that the sensitivity shares the same condition as the focusing quality, stated in section 7.4; suggesting TREF as a figure of merit for the sensitivity. However, the simulations do not confirm it. The simulation results indicate that the sensitivity is more complex. It is ruled by the sensitivity of each participating mode, as theoretically examined in Chapter 5 . The presence of a high sensitivity mode can enhance the amplitude decrease without, necessarily, enlarging TREF. However, a final conclusion could not be drawn because the sensitivity of the $A 0$ mode is compromised in the simulations, as observed in the paragraph above. Further investigation on this relationship is needed. 


\section{0}

\section{Conclusion}

By means of the acoustoelastic theory and through the partial wave technique, dispersion curves can be calculated for Lamb wave under stress. This can be done either at natural or initial frame of reference, if the precise derivation is done considering the asymmetries in the stiffness tensor induced by the load. The choice of the frame of reference relies on the treated problem. Velocities are dependent on the initial stress. The most expressive way of reporting this dependence is by the sensitivity chart, as reported in the Fig. 5.7 at the natural frame of reference and in Fig. 5.10 at the initial one. The velocity variation at natural and initial frame cannot be related by the same expression used for bulk waves, however this expression is valid for low dispersive regions.

The use of an equivalent anisotropic stiffness tensor by effective elastic constants, introduces errors when compared with the exact solution. These errors depend on frequency and mode as clearly pointed out in Fig. 5.21 and 5.22. This approximation is however useful, as for numerical simulation, for instance. By adopting effective elastic constants it is possible to simulate loaded medium in commercial programs simply by treating them as an anisotropic medium. Finite element analysis in commercial software for Lamb waves modes has been validated confronting the theoretical curves for A0 and S0 modes in a longitudinally loaded plate. It has been demonstrated that the FEM results correspond to the theoretical dispersion curves obtained for the respective EEC.

This approach allow investigating the load effect in ultrasonic waves in complex media, such as complicated geometries, non-homogeneity and bodies with defects, for instance, where closed form analytical models are not available. It also allows to numerically investigate the load effect on the time reversal process of wideband multi-mode Lamb waves, specially on its focus. This was done here.

Two main effects are observable in the time reversal focus, peak amplitude decrease and time-of-flight shift. There is an intrinsic trade off between them. If peak decrease is accentuated, then it may not be possible to detect its precise instant and thus time-of-flight measurement becomes unfeasible. Results show that the sensitivity is very depended on the complexity of the medium and 
the nature of propagation. This is due to the fact that the focus changes are ruled by the mismatch, between forward and backward propagation, of each component present in the signals. If all components have the same phase sensitivity thus only time-of-flight shift occurs. Otherwise, if each component present different phase sensitivities, then the mismatch is manifested also as peak decrease. The peak amplitude behavior as a function of load showed to be similar to the spatial focus pattern. When end reflections are detectable the sensitivity is much higher because the reflections act as a mode multiplier increasing the complexity of the medium.

The effect of using the EEC approximation is more serious in the timeshift than in peak decrease. Amplitude decrease of wideband propagation shows the same behavior with EEC or with exactly dispersion curves, because about the same relative mismatch between components are present in both version. This was verified by using the analytical model with estimated modes intensity.

Experiments were performed with three different 2-2 piezocomposite transducers. Despite of the intrinsic differences between experiments and simulations pointed out in section 9.5, the main behavior does agree. This suggest that the methodology proposed for simulations is able to reproduce the effects of load in guided wave propagation, specially its influence on the time reversal process.

The one-bit simplification is possible in more complex cases, as finite length plate. Where almost the same results were obtained; actually a little increase in sensitivity is present in this implementation. For simpler cases, as infinite medium with few modes, the sparsity in the signal makes the one-bit correlation to poorly match the medium response and thus the obtained time reversal signal may not focus. For practical applications in the complex media the one-bit variation reveals an important enhancement for the methodology. Besides requiring simpler hardware, it presented higher SNR (about $11 \mathrm{~dB}$ ) and sensitivity. The correlation approach was also verified to be equivalent to the physical implementation of the time reversal, either in simulations as in experiments.

The experimental results show improvements in the feasibility of the method when compared with previous work using a single commercial transducer [53]. The monoelement transducer provided a sensitivity of $0.041 \% / \mu \mathrm{m} / \mathrm{m}$. A greater sensitivity was achieved here; up to about $0.120 \% / \mu \mathrm{m} / \mathrm{m}$ for the highest sensitivity obtained, and the amplitude level of the focus is up to tenfold higher, increasing $19 \mathrm{~dB}$ in SNR, when using the conventional time reversal setup. For the case of one-bit tim reversal, the SNR 
is increased by $30 \mathrm{~dB}$.

For low strains both time-of-flight and amplitude reduction of the time reversal signal are sensitive to strain. However, time-of-flight shift observation requires high sampling frequency due to the order of magnitude of the sensitivity. Sampling rates as high as $200 \mathrm{MHz}$ [164] up to $1 \mathrm{GHz}$ [167] are used in the literature. Here a low pass interpolation was used to obtain a resolution of about $1 \mathrm{~ns}$ using a sampling frequency of $10 \mathrm{M} \mathrm{Hz}$. Moreover, the amplitude sensitivity is technically simpler to be implemented, as a voltage resolution of the order of a few hundredths of percent is easier to obtain than a $\mathrm{GHz}$ sampling frequency. Therefore, for practical applications, amplitude observation at a low sampling rate is a desired feature for the method, and higher amplitude sensitivity is an important property.

For high strains, peak measurement is not possible as the peak totally vanishes. In this case it is recommended measuring by time-shift observation by single mode propagation, as done in former works [24, 103-107, 164, 167]. Nevertheless, peak value observation for high stress has a practical importance to detect sudden changes in the strain level in order to predict mechanical breaks in systems subjected to high loads. As in this case, the peak of the focused signal would totally vanish, as it was shown by the simulations. A simple threshold detector can be used to trigger an alarm, for example. Thus, it is feasible to use it for structural failure detection applications, for instance, breaking of riser wires.

\section{1}

\section{Future works}

Several works can be suggested as continuation of this study. The most relevant are summarized below.

- Investigate a non-reflective experimental setup in order to confirm the simulated results. Special attentions should be drawn to the time delay, in order to analyze if its order of magnitude will be closer to simulation.

- Perform a more realistic simulation by including the transducer into the model. This can be done by including a piezoelectric element in the software (option PIEZ in Ref. [127] sec. 2.5.7). In addition, to provide a more realistic simulation, with the proper transducer characteristics as its bandwidth, for instance, this would allow to investigate the possible reflections on the bonded transducer. A bonded mass on the plate surface reflect incident waves and causes mode conversion [17,60,168-170]. This is more relevant for the finite length plate were multiple reflections between the plate end and the transducer can be detected. 
- As a variation of the above item, one can simulate the effect of an EMAT transducer [171] in the FEM [31]. In this case acoustoelasticity should be addressed at the initial frame of reference as the distance between the transducers would not depend on the initial strain [105].

- Validate the acoustoelastic simulation also for finite-difference programs as Simsonic [172]. Simsonic is a free software specially designed for timedomain ultrasonic simulations in either two or three dimension.

- The influence of the geometric complexity of the medium on the time reversal focus characteristics and sensitivity can be studied by nonregular bodies as plates with holes or different thickness along its length. This can be done either in simulation or by experiments.

- A more representative simulation can be performed by three-dimensional geometry where the finite plate width can be modeled. This also allows to simulate the guidance by the width.

- Using simulation software one can numerically study the stress effect on the polarization of Lamb waves. This can be done as a function of the propagating angle if three dimensional geometry is modeled. Something like a stress induced slowness diagram for Lamb modes can be then created.

- In this work just the influence of the stress state on the Lamb wave velocity was studied. However, it also depends on the temperature in a very similar fashion than the stress dependence. That is, different velocity sensitivity for each mode and frequency $[173,174]$. However, differently from the stress dependence, the temperature effect is isotropic $[175,176]$. One can then investigate the applicability of using two pairs of transducer on the plate surface positioned in such a way that their propagation paths have the same distance but are perpendicular. This would be a setup like the one used by Shi et al. [24]. As the temperature dependence is isotropic the time-of-flight in both paths should be the same regardless any temperature variation. The difference between the time-of-flights would be a function of the stress state only.

- A theoretical framework for the temperature influence was done by Dodson and Inman $[173,174]$. One could then combine Dodson-Inman's development with the development exposed here in order to assemble an equivalent stiffness tensor that is a function of temperature and stress. This would allow numerical simulations as the ones performed here. 
A

Verification of stiffness and acoustoelastic tensors symmetries

This appendix analyzes the possible symmetries on the second and third order stiffness tensors and on accoustoelastic tensors.

\section{A.1}

\section{Stiffness tensors}

The symmetries on the second and third order stiffness tensors are verified here for a naturally isotropic material. However, the symmetry relationship states are valid for a general anisotropic medium.

\section{A.1.1}

\section{Second order stiffness tensor symmetries}

As can be straightly observed the Kroenecker delta tensor (2.6) is symmetric, i.e.,

$$
\delta_{i j}=\delta_{j i}
$$

The I (2.7) tensor possesses the following symmetries

$$
I_{i j k l}=I_{j i k l}=I_{i j l k}=I_{k l i j},
$$

this can be verified computing the above $\mathbf{I}$ tensor permutations.

$$
\begin{aligned}
& I_{j i k l}=\frac{\delta_{j k} \delta_{i l}+\delta_{j l} \delta_{i k}}{2}=I_{i j k l}, \\
& I_{i j l k}=\frac{\delta_{i l} \delta_{j k}+\delta_{i k} \delta_{j l}}{2}=I_{i j k l}, \\
& I_{k l i j}=\frac{\delta_{k i} \delta_{l j}+\delta_{k j} \delta_{l i}}{2}=\frac{\delta_{i k} \delta_{j l}+\delta_{j k} \delta_{i l}}{2}=I_{i j k l}
\end{aligned}
$$

It is also evident that the $\delta$ product presented on the first term of equation (2.5) present the full symmetries as the $\mathbf{I}$ tensor. This implies that second order stiffness tensor possesses the well-known full symmetry [14],

$$
C_{i j k l}=C_{j i k l}=C_{i j l k}=C_{k l i j}
$$




\section{A.1.2}

\section{Third order stiffness tensor symmetries}

In order to analyze the symmetry on the third order stiffness tensor (3.3) it is convenient to separate the analysis on its terms. Let $\mathcal{I}$ be the third term tensor of (3.3), i.e.,

$$
\mathcal{I}_{i j k l m n}=\delta_{i k} I_{j l m n}+\delta_{i l} I_{j k m n}+\delta_{j k} I_{i l m n}+\delta_{j l} I_{i k m n},
$$

its symmetries are

$$
\begin{aligned}
& \mathcal{I}_{i j k l m n}=\mathcal{I}_{j l k l m n}=\mathcal{I}_{i j l k m n}=\mathcal{I}_{i j k l n m}=\mathcal{I}_{\text {klijmn }} \quad \text { and } \\
& \mathcal{I}_{i j k l m n}=\mathcal{I}_{\text {ijmnkl }}=\mathcal{I}_{\text {mnklij }} .
\end{aligned}
$$

This can be verified computing the above permutations as,

$$
\begin{aligned}
\mathcal{I}_{j i k l m n} & =\delta_{j k} I_{i l m n}+\delta_{j l} I_{i k m n}+\delta_{i k} I_{j l m n}+\delta_{i l} I_{j k m n}=\mathcal{I}_{i j k l m n}, \\
\mathcal{I}_{i j l k m n} & =\delta_{i l} I_{j k m n}+\delta_{i k} I_{j l m n}+\delta_{j l} I_{i k m n}+\delta_{j k} I_{i l m n}=\mathcal{I}_{i j k l m n}, \\
\mathcal{I}_{i j k l n m} & =\delta_{i k} I_{j l n m}+\delta_{i l} I_{j k n m}+\delta_{j k} I_{i l n m}+\delta_{j l} I_{i k n m} \\
& =\delta_{i k} I_{j l m n}+\delta_{i l} I_{j k m n}+\delta_{j k} I_{i l m n}+\delta_{j l} I_{i k m n}=\mathcal{I}_{i j k l m n} \\
\mathcal{I}_{k l i j m n} & =\delta_{k i} I_{l j m n}+\delta_{k j} I_{l i m n}+\delta_{l i} I_{k j m n}+\delta_{l j} I_{k i m n} \\
& =\delta_{i k} I_{j l m n}+\delta_{j k} I_{i l m n}+\delta_{i l} I_{j k m n}+\delta_{j l} I_{i k m n}=\mathcal{I}_{i j k l m n}
\end{aligned}
$$

To see the remainder symmetries one needs to unpack the tensor $\mathbf{I}$ inside $\mathcal{I}$ as

$$
\begin{aligned}
\mathcal{I}_{i j k l m n}= & \frac{1}{2}\left[\delta_{i k}\left(\delta_{j m} \delta_{l n}+\delta_{j n} \delta_{l m}\right)+\delta_{i l}\left(\delta_{j m} \delta_{k n}+\delta_{j n} \delta_{k m}\right)\right. \\
& \left.+\delta_{j k}\left(\delta_{i m} \delta_{l n}+\delta_{i n} \delta_{l m}\right)+\delta_{j l}\left(\delta_{i m} \delta_{k n}+\delta_{i n} \delta_{k m}\right)\right],
\end{aligned}
$$

thus the symmetries on the second line of (A.6) can be verified:

$$
\begin{aligned}
\mathcal{I}_{i j m n k l}= & \frac{1}{2}\left[\delta_{i m}\left(\delta_{j k} \delta_{n l}+\delta_{j l} \delta_{n k}\right)+\delta_{i n}\left(\delta_{j k} \delta_{m l}+\delta_{j l} \delta_{m k}\right)\right. \\
& \left.+\delta_{j m}\left(\delta_{i k} \delta_{n l}+\delta_{i l} \delta_{n k}\right)+\delta_{j n}\left(\delta_{i k} \delta_{m l}+\delta_{i l} \delta_{m k}\right)\right] \\
= & \frac{1}{2}\left[\delta_{i k}\left(\delta_{j m} \delta_{n l}+\delta_{j n} \delta_{m l}\right)+\delta_{i l}\left(\delta_{j m} \delta_{n k}+\delta_{j n} \delta_{m k}\right)\right. \\
& \left.+\delta_{j k}\left(\delta_{i m} \delta_{n l}+\delta_{i n} \delta_{m l}\right)+\delta_{j l}\left(\delta_{i m} \delta_{n k}+\delta_{i n} \delta_{m k}\right)\right] \\
= & \frac{1}{2}\left[\delta_{i k}\left(\delta_{j m} \delta_{l n}+\delta_{j n} \delta_{l m}\right)+\delta_{i l}\left(\delta_{j m} \delta_{k n}+\delta_{j n} \delta_{k m}\right)\right. \\
& \left.+\delta_{j k}\left(\delta_{i m} \delta_{l n}+\delta_{i n} \delta_{l m}\right)+\delta_{j l}\left(\delta_{i m} \delta_{k n}+\delta_{i n} \delta_{k m}\right)\right]=\mathcal{I}_{i j k l m n}
\end{aligned}
$$




$$
\begin{aligned}
\mathcal{I}_{m n k l i j}= & \frac{1}{2}\left[\delta_{m k}\left(\delta_{n i} \delta_{l j}+\delta_{n j} \delta_{l i}\right)+\delta_{m l}\left(\delta_{n i} \delta_{k j}+\delta_{n j} \delta_{k i}\right)\right. \\
& \left.+\delta_{n k}\left(\delta_{m i} \delta_{l j}+\delta_{m j} \delta_{l i}\right)+\delta_{n l}\left(\delta_{m i} \delta_{k j}+\delta_{m j} \delta_{k i}\right)\right] \\
= & \frac{1}{2}\left[\delta_{k i}\left(\delta_{m j} \delta_{n l}+\delta_{n j} \delta_{m l}\right)+\delta_{l i}\left(\delta_{m j} \delta_{n k}+\delta_{n j} \delta_{m k}\right)\right. \\
& \left.+\delta_{k j}\left(\delta_{m i} \delta_{n l}+\delta_{n i} \delta_{m l}\right)+\delta_{l j}\left(\delta_{m i} \delta_{n k}+\delta_{n i} \delta_{m k}\right)\right] \\
= & \frac{1}{2}\left[\delta_{i k}\left(\delta_{j m} \delta_{l n}+\delta_{j n} \delta_{l m}\right)+\delta_{i l}\left(\delta_{j m} \delta_{k n}+\delta_{j n} \delta_{k m}\right)\right. \\
& \left.+\delta_{j k}\left(\delta_{i m} \delta_{l n}+\delta_{i n} \delta_{l m}\right)+\delta_{j l}\left(\delta_{i m} \delta_{k n}+\delta_{i n} \delta_{k m}\right)\right]=\mathcal{I}_{i j k l m n}
\end{aligned}
$$

Let $\mathbb{I}$ be the second term tensorof (3.3), i.e.,

$$
\mathbb{I}_{i j k l m n}=\delta_{i j} I_{k l m n}+\delta_{k l} I_{m n i j}+\delta_{m n} I_{i j k l}
$$

It presents the same symmetries as the precedent tensor, i.e.,

$$
\begin{aligned}
& \mathbb{I}_{i j k l m n}=\mathbb{I}_{j i k l m n}=\mathbb{I}_{i j l k m n}=\mathbb{I}_{i j k l n m}=\mathbb{I}_{k l i j m n} \quad \text { and } \\
& \mathbb{I}_{i j k l m n}=\mathbb{I}_{i j m n k l}=\mathbb{I}_{m n k l i j}
\end{aligned}
$$

This can be verified as,

$$
\begin{aligned}
\mathbb{I}_{j i k l m n} & =\delta_{j i} I_{k l m n}+\delta_{k l} I_{m n j i}+\delta_{m n} I_{j i k l} \\
& =\delta_{i j} I_{k l m n}+\delta_{k l} I_{m n i j}+\delta_{m n} I_{i j k l}=\mathbb{I}_{i j k l m n}, \\
\mathbb{I}_{i j l k m n} & =\delta_{i j} I_{l k m n}+\delta_{l k} I_{m n i j}+\delta_{m n} I_{i j l k} \\
& =\delta_{i j} I_{k l m n}+\delta_{k l} I_{m n i j}+\delta_{m n} I_{i j k l}=\mathbb{I}_{i j k l m n}, \\
\mathbb{I}_{i j k l n m} & =\delta_{i j} I_{k l n m}+\delta_{k l} I_{n m i j}+\delta_{n m} I_{i j k l} \\
& =\delta_{i j} I_{k l m n}+\delta_{k l} I_{m n i j}+\delta_{m n} I_{i j k l}=\mathbb{I}_{i j k l m n}, \\
\mathbb{I}_{k l i j n m} & =\delta_{k l} I_{i j m n}+\delta_{i j} I_{m n k l}+\delta_{m n} I_{k l i j} \\
& =\delta_{k l} I_{m n i j}+\delta_{i j} I_{k l m n}+\delta_{m n} I_{i j k l}=\mathbb{I}_{i j k l m n}, \\
\mathbb{I}_{i j m n k l} & =\delta_{i j} I_{m n k l}+\delta_{m n} I_{k l i j}+\delta_{k l} I_{i j m n} \\
& =\delta_{i j} I_{k l m n}+\delta_{m n} I_{i j k l}+\delta_{k l} I_{m n i j}=\mathbb{I}_{i j k l m n}, \\
\mathbb{I}_{m n k l i j} & =\delta_{m n} I_{k l i j}+\delta_{k l} I_{i j m n}+\delta_{i j} I_{m n k l} \\
& =\delta_{m n} I_{i j k l}+\delta_{k l} I_{m n i j}+\delta_{i j} I_{k l m n}=\mathbb{I}_{i j k l m n},
\end{aligned}
$$

At the first term of (3.3), the triple $\delta$ product, present the same symmetries as can be straightforward observed. Concluding that the third order stiffness tensor possesses the symmetries

$$
\begin{aligned}
& C_{i j k l m n}=C_{j i k l m n}=C_{i j l k m n}=C_{i j k l n m}=C_{k l i j m n} \quad, \text { and } \\
& C_{i j k l m n}=C_{i j m n k l}=C_{m n k l i j} .
\end{aligned}
$$




\section{A. 2}

\section{Symmetries on acoustoelastic tensors}

The symmetries on the acoustoelastic tensors are carried out for a general anisotropic medium. This is done from the symmetries on the stiffness tensors.

In order to analyze the symmetry on the tensors $\mathbf{A}^{n}, \mathbf{B}^{n}, \boldsymbol{\Gamma}, \mathbf{A}^{i}$ and $\mathbf{B}^{i}$, it is convenient to observe that, if a tensor presents some part that is known to exhibit the full symmetry condition (let it be called the full symmetrical part), then it is sufficient to analyze the symmetry of the remainder part. The symmetry of whole tensor is equal to the symmetry of the remainder part, because the full symmetrical part does not break any symmetry nor compensate any lack of it on the remainder part.

The tensor $\mathbf{B}^{i}$ (3.39) possesses the full symmetry. To conclude this one can observe that the first and last two terms on (3.39) possesses the full symmetry, i.e., they are the symmetrical part of $\mathbf{B}^{i}$. The remainder terms should be analyzed together. Let them be $\overline{\mathbf{B}}^{i}$

$$
\bar{B}_{I J K L}^{i}=C_{M J K L} \frac{\partial u_{I}^{i}}{\partial X_{M}}+C_{I M K L} \frac{\partial u_{J}^{i}}{\partial X_{M}}+C_{I J M L} \frac{\partial u_{K}^{i}}{\partial X_{M}}+C_{I J K M} \frac{\partial u_{L}^{i}}{\partial X_{M}} .
$$

The symmetries are verified considering the known symmetries on $C_{i j k l}$ from (A.4):

$$
\begin{aligned}
\bar{B}_{J I K L}^{i} & =C_{M I K L} \frac{\partial u_{J}^{i}}{\partial X_{M}}+C_{J M K L} \frac{\partial u_{I}^{i}}{\partial X_{M}}+C_{J I M L} \frac{\partial u_{K}^{i}}{\partial X_{M}}+C_{J I K M} \frac{\partial u_{L}^{i}}{\partial X_{M}} \\
& =C_{I M K L} \frac{\partial u_{J}^{i}}{\partial X_{M}}+C_{M J K L} \frac{\partial u_{I}^{i}}{\partial X_{M}}+C_{I J M L} \frac{\partial u_{K}^{i}}{\partial X_{M}}+C_{I J K M} \frac{\partial u_{L}^{i}}{\partial X_{M}}=\bar{B}_{I J K L}^{i}, \\
\bar{B}_{I J L K}^{i} & =C_{M J L K} \frac{\partial u_{I}^{i}}{\partial X_{M}}+C_{I M L K} \frac{\partial u_{J}^{i}}{\partial X_{M}}+C_{I J M K} \frac{\partial u_{L}^{i}}{\partial X_{M}}+C_{I J L M} \frac{\partial u_{K}^{i}}{\partial X_{M}} \\
& =C_{M J K L} \frac{\partial u_{I}^{i}}{\partial X_{M}}+C_{I M K L} \frac{\partial u_{J}^{i}}{\partial X_{M}}+C_{I J K M} \frac{\partial u_{L}^{i}}{\partial X_{M}}+C_{I J M L} \frac{\partial u_{K}^{i}}{\partial X_{M}}=\bar{B}_{I J K L}^{i}, \\
\bar{B}_{K L I J}^{i} & =C_{M L I J} \frac{\partial u_{K}^{i}}{\partial X_{M}}+C_{K M I J} \frac{\partial u_{L}^{i}}{\partial X_{M}}+C_{K L M J} \frac{\partial u_{I}^{i}}{\partial X_{M}}+C_{K L I M} \frac{\partial u_{J}^{i}}{\partial X_{M}} \\
& =C_{I J M L} \frac{\partial u_{K}^{i}}{\partial X_{M}}+C_{I J K M} \frac{\partial u_{L}^{i}}{\partial X_{M}}+C_{M J K L} \frac{\partial u_{I}^{i}}{\partial X_{M}}+C_{I M K L} \frac{\partial u_{J}^{i}}{\partial X_{M}}=\bar{B}_{I J K L}^{i},
\end{aligned},
$$

Thus, both $\overline{\mathbf{B}}^{i}$ and $\mathbf{B}^{i}$ possess the full symmetry, i.e.,

$$
B_{I J K L}^{i}=B_{J K L}^{i}=B_{I J L K}^{i}=B_{K L I J}^{i}
$$

In the tensor $\mathbf{A}^{i}$ (3.41) the second term is just the stiffness times a constant and thus present the full symmetry. Let $\overline{\mathbf{A}}^{i}$ be the first term, it clearly does not present the symmetries on $I J$ nor $K L$. However, the third symmetry holds, as can be verified.

$$
\bar{A}_{K L I J}^{i}=C_{L J M N} \varepsilon_{M N} \delta_{K I}=C_{J L M N} \varepsilon_{M N} \delta_{I K}=\bar{A}_{I J K L}^{i}
$$


Concluding that the tensor $\mathbf{A}^{i}$ presents just the symmetry

$$
\begin{aligned}
& A_{I J K L}^{i}=A_{K L I J}^{i} \quad, \text { and in general } \\
& A_{I J K L}^{i} \neq A_{J I K L}^{i} \neq A_{I J L K}^{i} .
\end{aligned}
$$

Differently from the tensor $\mathbf{B}^{i}$, the tensor $\boldsymbol{\Gamma}$ (3.31) presents just the following symmetry

$$
\begin{aligned}
& \Gamma_{\alpha \beta \gamma \delta}=\Gamma_{\gamma \delta \alpha \beta} \quad, \text { and in general } \\
& \Gamma_{\alpha \beta \gamma \delta} \neq \Gamma_{\beta \alpha \gamma \delta} \neq \Gamma_{\alpha \beta \delta \gamma} .
\end{aligned}
$$

To conclude this, one can observe that the first and last terms on (3.31) possesses the full symmetry. The remaining terms should be analyzed together. Let them be $\overline{\boldsymbol{\Gamma}}$

$$
\bar{\Gamma}_{\alpha \beta \gamma \delta}=C_{\alpha \beta \lambda \delta} \frac{\partial u_{\gamma}^{i}}{\partial \xi_{\lambda}}+C_{\lambda \beta \gamma \delta} \frac{\partial u_{\alpha}^{i}}{\partial \xi_{\lambda}}
$$

The symmetry is verified as

$$
\begin{aligned}
\bar{\Gamma}_{\gamma \delta \alpha \beta} & =C_{\gamma \delta \lambda \beta} \frac{\partial u_{\alpha}^{i}}{\partial \xi_{\lambda}}+C_{\lambda \delta \alpha \beta} \frac{\partial u_{\gamma}^{i}}{\partial \xi_{\lambda}} \\
& =C_{\lambda \beta \gamma \delta} \frac{\partial u_{\alpha}^{i}}{\partial \xi_{\lambda}}+C_{\alpha \beta \lambda \delta} \frac{\partial u_{\gamma}^{i}}{\partial \xi_{\lambda}}=\bar{\Gamma}_{\alpha \beta \gamma \delta}
\end{aligned}
$$

The other symmetries are not satisfied due to the lack of the other terms, that are present at $\mathbf{B}^{i}$, necessary for equalizing the permutations, concluding thus (A.20).

The first term in $\mathbf{A}^{n}$ (3.36) is identical to that at $\mathbf{A}^{i}$ (3.41), confirming the symmetry condition of $\boldsymbol{\Gamma}^{n}$ (3.31) on $\mathbf{A}^{n}$ (3.31), concluding that

$$
\begin{aligned}
& A_{\alpha \beta \gamma \delta}^{n}=A_{\gamma \delta \alpha \beta}^{n} \quad, \text { and in general } \\
& A_{\alpha \beta \gamma \delta}^{n} \neq A_{\beta \alpha \gamma \delta}^{n} \neq A_{\alpha \beta \delta \gamma}^{n} .
\end{aligned}
$$

However, the non-symmetrical part of $\mathbf{A}^{n}$ may combine to $\boldsymbol{\Gamma}^{n}$ and provide some more symmetry to $\mathbf{A}^{n}$. In sections A.2.1 a particular case where this happens is presented.

Observing $\mathbf{B}^{n}$ at (3.28) one can notice that the first and last term compose the full symmetrical part, and the middle term only presents symmetry on $\alpha \beta$, restricting it tensor to present

$$
\begin{aligned}
& B_{\alpha \beta \gamma \delta}^{n}=B_{\beta \alpha \gamma \delta}^{n} \quad, \text { and in general } \\
& B_{\alpha \beta \gamma \delta}^{n} \neq B_{\alpha \beta \delta \gamma}^{n} \neq B_{\gamma \delta \alpha \beta}^{n} .
\end{aligned}
$$




\section{A.2.1}

\section{Acoustoelastic tensors for naturally isotropic medium}

Considering that the medium is isotropic in it natural unstressed state and that the null rotation condition on strain holds (3.43), the symmetries on the tensors can be extended. It is demonstrated here that, in this case, the tensor $\mathbf{A}^{n}$ possesses the following symmetries

$$
\begin{aligned}
& A_{\alpha \beta \gamma \delta}^{n}=A_{\gamma \delta \alpha \beta}^{n} \quad, \\
& A_{\alpha \beta \gamma \delta}^{n}=A_{\beta \alpha \delta \gamma}^{n} \quad, \text { but in general } \\
& A_{\alpha \beta \gamma \delta}^{n} \neq A_{\beta \alpha \gamma \delta}^{n} \neq A_{\alpha \beta \delta \gamma}^{n} .
\end{aligned}
$$

The other tensor symmetries are the same.

To conclude that it is convenient to write $\mathbf{A}^{n}$ as

$$
A_{\alpha \beta \gamma \delta}^{n}=\tilde{A}_{\alpha \beta \gamma \delta}^{n}+\tilde{\tilde{A}}_{\alpha \beta \gamma \delta}^{n},
$$

where

$$
\tilde{A}_{\alpha \beta \gamma \delta}^{n}=C_{\beta \delta \zeta \eta} \varepsilon_{\zeta \eta} \delta_{\alpha \gamma}+C_{\alpha \beta \lambda \delta} \varepsilon_{\gamma \lambda}+C_{\lambda \beta \gamma \delta} \varepsilon_{\alpha \lambda}
$$

and

$$
\tilde{\tilde{A}}_{\alpha \beta \gamma \delta}^{n}=C_{\alpha \beta \gamma \delta}+C_{\alpha \beta \lambda \delta \zeta \eta} \varepsilon_{\zeta \eta}
$$

The $\tilde{\tilde{\mathbf{A}}}^{n}$ is easily identified as the full symmetrical part, i.e., possessing the full symmetries. $\tilde{\mathbf{A}}^{n}$ is analyzed considering the material as naturally isotropic, i.e., replacing the fourth order stiffness tensor by (2.5). Developing it, leads to

$$
\begin{aligned}
\tilde{A}_{\alpha \beta \gamma \delta}^{n} & =\left[\lambda \delta_{\beta \delta} \delta_{\zeta \eta}+\mu\left(\delta_{\beta \zeta} \delta_{\delta \eta}+\delta_{\beta \eta} \delta_{\delta \zeta}\right)\right] \varepsilon_{\zeta \eta} \delta_{\alpha \gamma} \\
& +\left[\lambda \delta_{\alpha \beta} \delta_{\lambda \delta}+\mu\left(\delta_{\alpha \lambda} \delta_{\beta \delta}+\delta_{\alpha \delta} \delta_{\beta \lambda}\right)\right] \varepsilon_{\gamma \lambda} \\
& +\left[\lambda \delta_{\lambda \beta} \delta_{\gamma \delta}+\mu\left(\delta_{\lambda \gamma} \delta_{\beta \delta}+\delta_{\lambda \delta} \delta_{\beta \gamma}\right)\right] \varepsilon_{\alpha \lambda} \\
& =\lambda \delta_{\beta \delta} \delta_{\alpha \gamma} \varepsilon_{\zeta \zeta}+\mu\left(\delta_{\alpha \gamma} \varepsilon_{\beta \delta}+\delta_{\alpha \gamma} \varepsilon_{\delta \beta}\right) \\
& +\lambda \delta_{\alpha \beta} \varepsilon_{\gamma \delta}+\mu\left(\delta_{\beta \delta} \varepsilon_{\gamma \alpha}+\delta_{\alpha \delta} \varepsilon_{\gamma \beta}\right) \\
& +\lambda \delta_{\gamma \delta} \varepsilon_{\alpha \beta}+\mu\left(\delta_{\beta \delta} \varepsilon_{\alpha \gamma}+\delta_{\beta \gamma} \varepsilon_{\alpha \delta}\right) \\
& =\lambda \varepsilon_{\zeta \zeta} \delta_{\beta \delta} \delta_{\alpha \gamma}+\lambda\left(\delta_{\alpha \beta} \varepsilon_{\gamma \delta}+\delta_{\gamma \delta} \varepsilon_{\alpha \beta}\right) \\
& \left.+\mu\left[\delta_{\alpha \gamma}\left(\varepsilon_{\beta \delta}+\varepsilon_{\delta \beta}\right)+\delta_{\beta \delta}\left(\varepsilon_{\gamma \alpha}+\varepsilon_{\alpha \gamma}\right)+\delta_{\alpha \delta} \varepsilon_{\gamma \beta}+\delta_{\beta \gamma} \varepsilon_{\alpha \delta}\right)\right]
\end{aligned}
$$

The symmetry is verified

$$
\begin{aligned}
\tilde{A}_{\beta \alpha \delta \gamma}^{n} & =\lambda \varepsilon_{\zeta \zeta} \delta_{\alpha \gamma} \delta_{\beta \delta}+\lambda\left(\delta_{\beta \alpha} \varepsilon_{\delta \gamma}+\delta_{\delta \gamma} \varepsilon_{\beta \alpha}\right) \\
& \left.+\mu\left[\delta_{\beta \delta}\left(\varepsilon_{\alpha \gamma}+\varepsilon_{\gamma \alpha}\right)+\delta_{\alpha \gamma}\left(\varepsilon_{\delta \beta}+\varepsilon_{\beta \delta}\right)+\delta_{\beta \gamma} \varepsilon_{\delta \alpha}+\delta_{\alpha \delta} \varepsilon_{\beta \gamma}\right)\right]=\tilde{A}_{\alpha \beta \gamma \delta}^{n},
\end{aligned}
$$

where the symmetry on the strain tensor $\varepsilon$ is used on the last two terms. The other symmetries $\left(A_{\alpha \beta \gamma \delta}^{n} \neq A_{\beta \alpha \gamma \delta}^{n} \neq A_{\alpha \beta \delta \gamma}^{n}\right)$ still not holding because at least at the first term $\left(\delta_{\alpha \gamma} \delta_{\beta \delta}\right)$ they fail. Concluding (3.54).

The tensors $\mathbf{B}^{n}$ keeps its symmetry. To check it one can analyze its middle 
term (3.28), that is the unsymmetrical part of it, say,

$$
\tilde{B}_{\alpha \beta \gamma \delta}^{n}=C_{\alpha \beta \lambda \delta} \frac{\partial u_{\gamma}^{i}}{\partial \xi_{\lambda}}=C_{\alpha \beta \lambda \delta} \varepsilon_{\gamma \lambda}=\left[\lambda \delta_{\alpha \beta} \delta_{\lambda \delta}+\mu\left(\delta_{\alpha \lambda} \delta_{\beta \delta}+\delta_{\alpha \delta} \delta_{\beta \lambda}\right)\right] \varepsilon_{\gamma \lambda}
$$

that still presenting no other symmetries than (A.24).

The same is done to $\mathbf{A}^{i}$. Its symmetrical part is the tensor $\mathbf{B}^{i}$, that already present the full symmetry, and thus its unsymmetrical part is $\overline{\mathbf{A}}^{i}$, defined a (A.18). For the particular case it is written as

$$
\begin{aligned}
\bar{A}_{I J K L}^{i} & =C_{J L M N} \varepsilon_{M N} \delta_{I K} \\
& =\left[\lambda \delta_{J L} \delta_{M N}+\mu\left(\delta_{J M} \delta_{L N}+\delta_{J N} \delta_{L M}\right)\right] \varepsilon_{M N} \delta_{K I} \\
& =\lambda \delta_{J L} \delta_{K I} \varepsilon_{M M}+\mu \delta_{K I}\left(\varepsilon_{J L}+\varepsilon_{L J}\right)
\end{aligned}
$$

again only the symmetries on (A.19) are valid.

Concluding that when the medium is naturally isotropic there is an additional symmetry on $\mathbf{A}^{n}$, given by (A.25), that is not present on $\mathbf{A}^{i}$. 


\section{B \\ Publications}

In this appendix the published papers related to this work are listed.

One paper was published in Conference Proceedings in 2015. This is: Mechanical Strain Monitoring in Plates Using Wavelet Coherence Based Filter of Wideband Ultrasonic Guided Waves [57], which was presented by poster at the the 2015 ICU International Congress on Ultrasonics, Metz, France.

Two papers were published in Conference Proceedings in 2014. These are: Development of a mechanical strain sensor based on time reversal of ultrasonic guided waves [54] orally presented at 2014 IEEE International Instrumentation and Measurement Technology Conference, Montevideo, Uruguay, and Application of one-bit time reversal technique to mechanical strain monitoring in plates [55], presented by poster at the 2014 IEEE Ultrasonics Symposium, Chicago, USA.

The paper Mechanical strain sensing by broadband time reversal in plates [56] was published in the IEEE Transactions on Ultrasonics, Ferroelectrics, and Frequency Control. 


\section{Bibliography}

[1] BAI, Y.; BAI, Q. Subsea Pipelines and Risers. Elsevier, 2005.

[2] O'BRIEN, P.; PICKSLEY, J. State-of-the-Art Flexible Riser Integrity Issues. 2001. MCS International.

[3] SMITH, F. J.; O'BRIEN, P. ; PICKSLEY, J. Monitoring Methods and Integrity assurance for Unbonded Flexible Pipe (UKOOA Guidance Note). 2002. MCS International.

[4] MORIKAWA, S.; CAMERINI, C.; PIPA, D.; SANTOS, J.; PIRES, G.; BRAGA, A.; LLERENA, R. ; RIBEIRO, A. Monitoring of flexible oil lines using FBG sensors. In: PROCEEDINGS OF SPIE, volume 7004, p. $70046 \mathrm{~F}, 2008$.

[5] MORIKAWA, S.; BRAGA, A.M.B. CAMERINI, C.; KATO, C.; LlERENA, R. ; CAMERINI, M. New advances in flexible riser monitoring techniques using optical fiber sensors. In: ASME 2012 31ST INTERNATIONAL CONFERENCE ON OCEAN, OFFSHORE AND ARCTIC ENGINEERING, volume 3, p. 793-798, 2012.

[6] ANDERSEN, G. Well-integrity management and profitability. Journal of Petroleum Technology, p. 40-42, July 2006.

[7] Pinto, H. L. C.; BRAGA, A. M. B. ; GOUvÊA, P. M. P. Well integrity monitoring: Challenges and perspectives. In: OFFSHORE TECHNOLOGY CONFERENCE BRAZIL, p. 29-31, Oct 2013.

[8] HULL, J.; GOSSELIN, L. ; BORZEL, K. Well integrity monitoring $\&$ analysis using distributed acoustic fiber optic sensors. In: SPE DRILLING CONFERENCE AND EXHIBITION, NEW ORLEANS, LA, USA, p. 29-31, Feb 2010.

[9] LEE, J.-R.; RYU, C.-Y.; KOO, B.-Y.; KANG, S.-G.; HONG, C.-S. ; KIM, C.-G. In-flight health monitoring of a subscale wing using a fiber bragg grating sensor system. Smart Materials and Structures, 12(1):147, 2003. 
[10] MICHAELS, J. E.; MICHAELS, T. E. ; MARTIN, R. S. Analysis of global ultrasonic sensor data from a full scale wing panel test. In: AIP CONFERENCE PROCEEDINGS, 2009, p. 950-957, 2009.

[11] DI SANTE, R. Fibre optic sensors for structural health monitoring of aircraft composite structures: Recent advances and applications. Sensors, 15(8):18666-18713, Jul 2015.

[12] DE FREITAS, S. T.; KOLSTEIN, H. ; BIJLAARD, F. Structural monitoring of a strengthened orthotropic steel bridge deck using strain data. Struct. Health Monit., 11:558-576, 2012.

[13] MENG, W.; HONG, T. Design of stress monitoring and safety assessment system for crane metal structure based on virtual instrument. Appl. Mech. Mater., 152:1492-1497, 2012.

[14] ROSE, J. L. Ultrasonic waves in solid media. Cambridge University Press, 1999.

[15] SU, Z.; YE, L. Identification of Damage Using Lamb Waves. Lecture Notes in Applied and Computational Mechanics. 2009.

[16] RAGHAVAN, A.; CESNIK, C. E. S. Review of guided-waves structural health monitoring. The Shock and Vibration Digest, 39(2):91-114, 2007.

[17] IHN, J.; CHANG, F. Pitch-catch active sensing methods in structural health monitoring for aircraft structures. Struct. Health Monit., $7(1): 5-15,2008$.

[18] RATHOD, V.; MAHAPATRA, D. R. Ultrasonic lamb wave based monitoring of corrosion type of damage in plate using a circular array of piezoelectric transducers. NDT \& E International, 44(7):628 $-636,2011$.

[19] AMJAD, U.; TARAR, K. S.; SHELKE, A.; KUNDU, T.; PLUTA, M. ; GRILL, W. Generalized representations and universal aspects of lamb wave dispersion relations. volume 7650 , p. $76502 \mathrm{~F}-76502 \mathrm{~F}-8$, 2010 .

[20] VIKTOROV, L. Rayleigh and Lamb Waves. Plenum-Press, N.Y, 1970.

[21] HUGHES, D.; KELLY, J. Second-order elastic deformation of solids. J. Appl. Phys Phys Rev, 92:1145, 1953. 
[22] MISHAKIN, V. V.; DIXON, S. ; POTTER, M. D. G. The use of wide band ultrasonic signals to estimate the stress condition of materials. Journal of Physics D: Applied Physics, 39(21):4681, 2006.

[23] VANGI, D. Stress evaluation by pulse-echo ultrasonic longitudinal wave. Experimental Mechanics, 41(3):277-281, 2001.

[24] SHI, F.; MICHAELS, J. E. ; LEE, S. J. In situ estimation of applied biaxial loads with lamb waves. The Journal of the Acoustical Society of America, 133(2):677-687, 2013.

[25] BOWleR, A. I.; DRINKWATER, B. W. ; WILCOX, P. D. An investigation into the feasibility of internal strain measurement in solids by correlation of ultrasonic images. Proceedings of the Royal Society of London A: Mathematical, Physical and Engineering Sciences, 2011.

[26] MI, B.; MICHAELS, J. E. ; MICHAELS, T. E. An ultrasonic method for dynamic monitoring of fatigue crack initiation and growth. The Journal of the Acoustical Society of America, 119(1):74-85, 2006.

[27] DuquennoY, M.; OUAFTOUH, M.; OURAK, M. ; XU, W.-J. Influence of natural and initial acoustoelastic coefficients on residual stress evaluation: Theory and experiment. Journal of Applied Physics, 86(5):2490-2498, 1999.

[28] MOSER, F.; JACOBS, L. J. ; QU, J. Modeling elastic wave propagation in waveguides with the finite element method. NDT and E International, 32(4):225 - 234, 1999.

[29] Duquennoy, M.; OUAFtouh, M.; Devos, D.; JenOt, F. ; OURAK, M. Effective elastic constants in acoustoelasticity. Applied Physics Letters, 92(24):-, 2008.

[30] PEI, C.; DEMACHI., K. Numerical simulation of residual stress measurement with acoustic wave. E-journal of Advanced Maintenance, 2:160-167, 2011.

[31] FERRARI, R. M. G. The acoustoelastic effect: Emat excitation and reception of lamb waves in pre-stressed metal sheets. In: PROCEEDINGS OF 2009 COMSOL CONFERENCE, STUTTGART, 2009.

[32] LOVEDAY, P. W. Semi-analytical finite element analysis of elastic waveguides subjected to axial loads. Ultrasonics, 49(3):298 - 300, 2009. 
[33] CHEN, F.; WILCOX, P. D. The effect of load on guided wave propagation. Ultrasonics, 47(1-4):111 - 122, 2007.

[34] GANDHI, N.; MICHAELS, J. E. ; LEE, S. J. Acoustoelastic lamb wave propagation in biaxially stressed plates. The Journal of the Acoustical Society of America, 132(3):1284-1293, 2012.

[35] MURNAGHAN, F. D. Finite Deformation of an Elastic Solid. John Wiley \& Sons Inc., 1951.

[36] SMITH, R. T.; STERN, R. ; STEPHENS, R. W. B. Third-order elastic moduli of polycrystalline metals from ultrasonic velocity measurements. The Journal of the Acoustical Society of America, 40(5):1002-1008, 1966.

[37] ASAY, J.; GUENTHER, A. Ultrasonic studies of 1060 and 6061-t6 aluminum. Journal of Applied Physics, 38:4086-4088, Sep 1967.

[38] THOMAS, J. F. Third-order elastic constants of aluminum. Phys. Rev., 175:955-962, Nov 1968.

[39] Dubuget, M.; EL GUERJOUMA, R.; DubOIS, S.; BABOUX, J. C. ; VINCENT, A. Characterization of the Non-Linear Elastic Properties of Aluminium Alloys Using Ultrasonic Evaluation under Load. Materials Science Forum, 217-222:951-956, 1996.

[40] STOBBE, D. M. Acoustoelasticity in 7075-t651 aluminum and dependence of third order elastic constants on fatigue damage. Master thesis, School of Mechanical Engineering, Georgia Institute of Technology, 2005 .

[41] MUIR, D. D.; MICHAELS, J. E.; MICHAELS, T. E.; THOMPSON, D. O. ; CHIMENTI, D. E. An ultrasonic angle beam method for determining third order elastic constants via acoustoelasticity measurements. In: AIP CONFERENCE PROCEEDINGS, volume 975, p. 1207, 2008.

[42] MUIR, D. D. One-Sided Ultrasonic Determination of Third Order Elastic Constants using Angle-Beam Acoustoelasticity Measurements. PhD dissertation, School of Electrical and Computer Engineering, Apr. 2009.

[43] KNAPP, C.; CARTER, G. The generalized correlation method for estimation of time delay. Acoustics, Speech and Signal Processing, IEEE Transactions on, 24(4):320-327, Aug 1976. 
[44] ASHRAF, M.; QAYYUM, H. High accuracy time of flight measurement using digital signal processing techniques for subsea applications. Journal of Signal and Information Processing, 2(4):330-335, 2011.

[45] L.SVILAINIS; DUMBRAVA, V. The time-of-flight estimation accuracy versus digitization parameters. Ultragarsas Journal, 63(1):12-17, 2008 .

[46] BERTAIX, V.; GARSON, J.; QUIEFFIN, N.; CATHELINE, S.; DEROSNY, J. ; FINK, M. Time-reversal breaking of acoustic waves in a cavity. American Journal of Physics, 72(10):1308-1311, 2004.

[47] JIN, Y.; O'DOnOUGHUE, N. ; MOURA, J. M. F. Position location by time reversal in communication networks. In: IEEE INTERNATIONAL CONFERENCE ON ACOUSTICS, SPEECH, AND SIGNAL PROCESSING, April 2008.

[48] FINK, M. Time reversal of ultrasonic fields-part I: Basic principles. IEEE Transactions on Ultrasonics, Ferroelectrics, and Frequency Control, 39(5):555-566, Sept. 1992.

[49] PARK, H. W.; KIM, S. B. ; SOHN, H. Understanding a time reversal process in lamb wave propagation. Wave Motion, 46:451-497, 2009.

[50] WATKINS, R.; JHA, R. A modified time reversal method for lamb wave based diagnostics of composite structures. Mechanical Systems and Signal Processing, 31(0):345 - 354, 2012.

[51] ING, R. K.; FINK, M. Time-reversed lamb waves. IEEE Transactions on Ultrasonics, Ferroelectrics, and Frequency Control, 45(4):1032-1043, July 1998.

[52] KuBrusly, A. C.; PÉREZ, N. A.; ADAMOWSKI, J. C. ; VON DER WEID, J. P. Strain monitoring in metallic plates using the time reversal focusing technique. In: ULTRASONICS SYMPOSIUM (IUS), 2012 IEEE INTERNATIONAL, p. 1-4, Oct 2012.

[53] KUBRUsly, A. C.; PÉREZ, N.; ADAMOWSKI, J. C. ; VON DER WEID, J. P. Strain sensitivity model for guided waves in plates using the time-reversal technique. Ultrasonics, Ferroelectrics and Frequency Control, IEEE Transactions on, 60(12):2566-2574, Dec 2013. 
[54] KUBRUSlY, A. C.; PÉREZ, N. A.; ADAMOWSKI, J. C.; OLIVEIRA, T. F.; BRAGA, A. M. B. ; VON DER WEID, J. P. Development of a mechanical strain sensor based on time reversal of ultrasonic guided waves. In: INSTRUMENTATION AND MEASUREMENT TECHNOLOGY CONFERENCE (I2MTC), 2014 IEEE INTERNATIONAL, p. 309-314, May 2014.

[55] KUBrusly, A. C.; BRAGA, A. M.; VON DER WEID, J. P.; PÉREZ, N.; ADAMOWSKI, J. C. ; OLIVEIRA, T. F. D. Application of one-bit time reversal technique to mechanical strain monitoring in plates. In: ULTRASONICS SYMPOSIUM (IUS), 2014 IEEE INTERNATIONAL, p. 1408-1411, Sept 2014.

[56] Kubrusly, A. C.; PÉREZ, N.; DE OliveirA, T. F.; ADAMOWSKI, J. C.; BRAGA, A. M. B. ; VON DER WEID, J. P. Mechanical strain sensing by broadband time reversal in plates. IEEE Transactions on Ultrasonics, Ferroelectrics, and Frequency Control, 63(5):746-756, May 2016.

[57] KUBRUSlY, A.; BRAGA, A.; PÉREZ, N.; ADAMOWSKI, J.; DE OLIVEIRA, T. ; VON DER WEID, J. Mechanical strain monitoring in plates using wavelet coherence based filter of wideband ultrasonic guided waves. Physics Procedia, 70:393 - 397, 2015. Proceedings of the 2015 IICU\} International Congress on Ultrasonics, Metz, France.

[58] ROSE, J. L. Ultrasonic Guided waves in solid media. Cambridge University Press, 2014.

[59] ACHENBACH, J. D. Wave propagation in elastic solids. NorthHolland. Amsterdam, 1975.

[60] CHO, Y.; PARK, J.-C. ; ROSE, J. L. A study on the guided wave mode conversion using self-calibrating technique. In: 15TH WORLD CONFERENCE ON NONDESTRUCTIVE TESTING ROMA (ITALY) 1521 OCTOBER 2000, Oct 2000.

[61] BRAGA, A. M. B.; HERMANN, G. Floquet waves in anisotropic periodically layered composites. J. Acoust. Soc. Am., 91(3):1211-1227, Mar. 1992.

[62] PAVlakovic, B.; LOWE, M.; Alleyne, D. ; CAWley, P. Disperse: A general purpose program for creating dispersion curves. In: Thompson, D.; Chimenti, D., editors, REVIEW OF PROGRESS IN 
QUANTITATIVE NONDESTRUCTIVE EVALUATION, volume 16 de Review of Progress in Quantitative Nondestructive Evaluation, p. 185-192. Springer US, 1997.

[63] HORA, P.; CERVENA, P. Determination of lamb wave dispersion curves by means of fourier transform. Applied and Computational Mechanics, 6:5-16, 2012.

[64] GÓMEZ, P.; FERNÁNDEZ, J. P. ; GARCÍA, P. D. Lamb waves and dispersion curves in plates and its applications in nde experiences using comsol multiphysics. In: PROCEEDINGS OF 2011 COMSOL CONFERENCE, STUTTGART, 2011.

[65] CASTRO-COLIN, M.; LOPEZ, J. ; OSEGUEDA, R. Identification of laser-induced lamb waves. Revista Mexicana de Fisica, 553(3):12-15, 2007.

[66] SACHSE, W.; PAO, Y. On the determination of phase and group velocities of dispersive waves in solids. Journal of Applied Physics, 49(8):4320-4327, 1978.

[67] NIETHAMMER, M.; JACOBS, L. J.; QU, J. ; JARZYNSKI, J. Timefrequency representation of lamb waves using the reassigned spectrogram. The Journal of the Acoustical Society of America, 107(5):L19 L24, 2000.

[68] WALTISBERG, D.; RAISUTIS, R. Group velocity estimation of lamb waves based on the wavelet transform. Ultragarsas Journal, 63(4):35-40, 2008.

[69] KUlESH, M.; HOLSCHNEIDER, M.; DIALlO, M. S.; XIE, Q. ; SCHERBAUM, F. Modeling of wave dispersion using continuous wavelet transforms. Pure and Applied Geophysics, 162(5):843-855, 2005.

[70] KULESH, M.; HOLSCHNEIDER, M.; OHRNBERGER, M. ; LÜCK, E. Modeling of wave dispersion using continuous wavelet transforms II: wavelet based frequency-velocity analysis. Pure and Applied Geophysics, 165(2):255-270, 2008.

[71] HOLSCHNEIDER, M.; DIALLO, M. S.; KULESH, M.; OHRNBERGER, M.; LÜCK, E. ; SCHERBAUM, F. Characterization of dispersive surface waves using continuous wavelet transforms. Geophysical Journal International, 163(2):463-478, 2005. 
[72] VEROY, K. P. L. Time-frequency analysis of lamb waves using the morlet wavelet transform. Master thesis, Massachusetts Institute of Technology. Department of Civil and Environmental Engineering., Feb 2000 .

[73] ASKARI, R.; FERGUSON, R. J. Estimation of dispersive parameters of surface wave using the generalized s transform. In: GEOCONVENTION 2013: INTEGRATION, p. 1-7, 2013.

[74] ASKARI, R.; FERGUSON, R. Dispersion and the dissipative characteristics of surface waves in the generalized s-transform domain. GEOPHYSICS, 77(1):V11-V20, 2012.

[75] PROSSER, W. H.; SEALE, M. D. ; SMITH, B. T. Time-frequency analysis of the dispersion of lamb modes. The Journal of the Acoustical Society of America, 105(5):2669-2676, 1999.

[76] OPPENHEIM, A. V.; WILlSKY, A. S. ; HAMID, S. Signals and Systems. Pretice Haal, 1996.

[77] Mallat, S. A Wavelet Tour of Signal Processing, Third Edition: The Sparse Way. Academic Press, 3 edition, Dec. 2008.

[78] SICARD, R.; GOYETTE, J. ; ZELLOUF, D. A numerical dispersion compensation technique for time recompression of lamb wave signals. Ultrasonics, 40(1-8):727 - 732, 2002.

[79] WILCOX, P. A rapid signal processing technique to remove the effect of dispersion from guided wave signals. Ultrasonics, Ferroelectrics, and Frequency Control, IEEE Transactions on, 50(4):419427, April 2003.

[80] XU, K.; TA, D.; MOILANEN, P. ; WANG, W. Mode separation of lamb waves based on dispersion compensation method. The Journal of the Acoustical Society of America, 131(4):2714-2722, 2012.

[81] YAMASAKI, T.; TAMAI, S. ; HIRAO, M. Optimum excitation signal for long-range inspection of steel wires by longitudinal waves. NDT \& E International, 34(3):207 - 212, 2001.

[82] XU, K.; TA, D.; HU, B.; LAUGIER, P. ; WANG, W. Wideband dispersion reversal of lamb waves. IEEE Trans Ultrason Ferroelectr Freq Control, 61(6):997-1005, 2014. 
[83] KIM, K. Y.; SACHSE, W. Acoustoelasticity of elastic solids in: Handbook of elastic properties of solids, liquids, and gases. Academic Press, 1:441-468, 2001.

[84] FU, Y. B.; OGDEN, R. W. Nonlinear Elasticity: Theory and Applications. Cambridge University Press, 2001.

[85] OGDEN, R. W. Non-Linear Elastic Deformations. Dover Civil and Mechanical Engineering, 1997.

[86] SHAMS, M.; DESTRADE, M. ; OGDEN, R. Initial stresses in elastic solids: Constitutive laws and acoustoelasticity. Wave Motion, 48(7):552 - 567, 2011.

[87] DE PASCALIS, R. The Semi-Inverse Method in solid mechanics: Theoretical underpinnings and novel applications. PhD dissertation, Université Pierre et Marie Curie - Università del Salento, 2010.

[88] ABIZA, Z.; DESTRADE, M. ; OGDEN, R. Large acoustoelastic effect. Wave Motion, 49(2):364 - 374, 2012.

[89] DeSTRADE, M.; GILCHRIST, M. D. ; SACCOMAnDI, G. Thirdand fourth-order constants of incompressible soft solids and the acousto-elastic effect. The Journal of the Acoustical Society of America, 127(5):2759-2763, 2010.

[90] TATSUO, T.; YUKIO, I. Acoustical birefringence of ultrasonic waves in deformed isotropic elastic materials. International Journal of Solids and Structures, 4(3):383 - 389, 1968.

[91] PAO, Y.-H.; GAMER, U. Acoustoelastic waves in orthotropic media. Acoustical Society of America Journal, 77:806-812, Mar. 1985.

[92] PAO, Y.; SACHSE, W. ; FUKUOKA, H. Acoustoelasticity and ultrasonic measurements of residual stresses. Physical Acoustics, 17:61-143, 1984.

[93] HUSSON, D.; KINO, G. S. A perturbation theory for acoustoelastic effects. Journal of Applied Physics, 53(11):7250-7258, 1982.

[94] HUSSON, D. A perturbation theory for the acoustoelastic effect of surface waves. Journal of Applied Physics, 57(5):1562-1568, 1985.

[95] LITTLE, R. W. Elasticity. Prentice-Hall, Inc., 1973. 
[96] GANDHI, N. Determination of dispersion curves for acoustoelastic lamb wave propagation. Master thesis, School of Electrical and Computer Engineering, Georgia Institute of Technology, 2010.

[97] FUCK, R. F.; TSVANKIN, I. Analysis of the symmetry of a stressed medium using nonlinear elasticity. GEOPHYSICS, 74(5):WB79WB87, 2009.

[98] DUQUENNOY, M.; OUAFTOUCH, M.; OURAK, M.; JENOT, F. ; XU, W.-J. Theoretical and experimental determination of the natural and initial acoustoelastic coefficients. AIP Conference Proceedings, 615(1):1696-1702, 2002.

[99] LOVEDAY, P. W.; WILCOX, P. D. Guided wave propagation as a measure of axial loads in rails. Proc. SPIE, 7650:765023-765023-8, 2010 .

[100] LOVEDAY, P. W.; LONG, C. S. ; WILCOX, P. D. Semi-analytical finite element analysis of the influence of axial loads on elastic waveguides. In: Moratal, D., editor, FINITE ELEMENT ANALYSIS FROM BIOMEDICAL APPLICATIONS TO INDUSTRIAL DEVELOPMENTS. InTech, 2012.

[101] PAU, A.; LANZA DI SCALEA, F. Nonlinear guided wave propagation in prestressed plates. The Journal of the Acoustical Society of America, 137(3):1529-1540, 2015.

[102] MOHABUTH, M.; KOTOUSOV, A. ; NG, C.-T. Lamb waves in plates subjected to uniaxial stresses. ICAMME 2015: International Conference on Applied Mechanics and Mechanical Engineering, Stockholm, Sweden, (Jul 13-14, 2015), 2(7):313, 2015.

[103] MURAYAMA, R.; TOKUnAGA, S. ; HIRATA, K. Development of a non-contacting stress measurement system during tensile testing using the electromagnetic acoustic transducer for a lamb wave. In: PROCEEDINGS OF THE 16TH WCNDT 2004 - WORLD CONFERENCE ON NDT, AUG 30 - SEP 3, 2004 - MONTREAL, CANADA, número 642, Sep. 2004.

[104] MURAYAM, R.; MISUMI, K. Development of a non-contact stress measurement system during tensile testing using the electromagnetic acoustic transducer for a lamb wave. NDT \& E International, 39(4):299 - 303, 2006. 
[105] MURAYAMA, R. Non-contact stress measurement during tensile testing using an emat for SH0-plate wave and lamb wave. Journal of Sensor Technology, 1(3):65 - 70, 2011.

[106] ZAGRAI, A.; GIGINEISHVILI, V.; KRUSE, W. A.; MURRAY, A.; DOYLE, D.; REYNOLDS, W.; ARRITT, B. ; GARDENIER, H. Acoustoelastic measurements and baseline-free assessment of bolted joints using guided waves in space structures. volume 7650, p. $765017-$ 765017-12, 2010.

[107] AMJAD, U.; JHA, D.; TARAR, K. S.; KLINGHAMMER, H. ; GRILL, W. Determination of the stress dependence of the velocity of lamb waves in aluminum plates. volume 7984, p. 798410-798410-9, 2011.

[108] KAYESTHA, P.; WIJEYEWICKREMA, A. C. ; KISHIMOTO, K. Wave propagation along a non-principal direction in a compressible pre-stressed elastic layer. International Journal of Solids and Structures, 48(14-15):2141 - 2153, 2011.

[109] WILLSON, A. J. Surface and plate waves in biaxially-stressed elastic media. Pure and Applied Geophysics, 102:182-192, Dec. 1973.

[110] NOLDE, E.; PRIKAZCHIKOVA, L. ; ROGERSON, G. Dispersion of small amplitude waves in a pre-stressed, compressible elastic plate. Journal of Elasticity, 75(1):1-29, 2004.

[111] OGDEN, R.; ROXBURGH, D. The effect of pre-stress on the vibration and stability of elastic plates. International Journal of Engineering Science, 31(12):1611 - 1639, 1993.

[112] ROGERSON, G. A.; FU, Y. B. An asymptotic analysis of the dispersion relation of a pre-stressed incompressible elastic plate. Acta Mechanica, 111(1):59-74, 1995.

[113] JONES, R. M. Mechanics of composite materials. Taylor \& Francis, 1999.

[114] GANDHI, N.; MICHAELS, J. E. ; LEE, S. J. Acoustoelastic lamb wave propagation in a homogeneous, isotropic aluminum plate. AIP Conference Proceedings, 1335(1):161-168, 2011.

[115] PRESS, W. H.; TEUKOLSKY, S. A.; VETTERLING, W. T. ; FLANNERY, B. P. Numerical Recipes in Fortran 77 The Art of Scientific Computing. Cambridge University Press, 1992. 
[116] OGDEN, R. Incremental statics and dynamics of pre-stressed elastic materials. In: Destrade, M.; Saccomandi, G., editors, WAVES IN NONLINEAR PRE-STRESSED MATERIALS, volume 495 de CISM Courses and Lectures, p. 1-26. Springer Vienna, 2007.

[117] NAYFEH, A. H.; CHIMENTI, D. E. Free Wave Propagation in Plates of General Anisotropic Media. Journal of Applied Mechanics, 56(4):881-886, 1989.

[118] VERMA, K. L. Wave propagation in plates of anisotropic media on the basis exact theory. Annals of the Faculty of Engineering Hunedoara - International J, 9(3):287-294, Sep 2011.

[119] THOMSON, W. T. Theory of Vibration with Applications. Prentice Hall, 1973.

[120] MOTT, G. Equations of elastic motion of an isotropic medium in the presence of body forces and static stresses. The Journal of the Acoustical Society of America, 50(3B):859-868, 1971.

[121] MOTT, G. Elastic waveguide propagation in an infinite isotropic solid cylinder that is subjected to a static axial stress and strain. The Journal of the Acoustical Society of America, 53(4):1129-1133, 1973.

[122] PAU, A.; LANZA DI SCALEA, F. Non-destructive evaluation of prestress in plates by nonlinear guided waves. In: PROCEEDINGS OF THE 9TH INTERNATIONAL CONFERENCE ON STRUCTURAL DYNAMICS, EURODYN 2014, p. 3887-38889, Jun. 2014.

[123] LIU, Y. Wave propagation study using finite element analysis. Master thesis, School of Electrical Engineering, 2005.

[124] NANDY, A.; MULLICK, S.; DE, S. ; DATTA, D. Numerical simulation of ultrasonic wave propagation in flawed domain. Indian National Seminar and Exhibition on Non-Destructive Evaluation NDE, p. $160-163,2009$.

[125] ANSYS® INC. Ansys ${ }^{\circledR}$ parametric design language guide, Nov 2011.

[126] MOAVENI, S. Finite Element Analysis Theory and Application with ANSYS. Prentice Hall, 1999.

[127] ANSYS® INC. Ansys® element reference, Nov 2009. 
[128] YAZDIZADEH, B. Comparison of Different Plane Models in Finite Element Software in Structural Mechanics. Proceedings of The Yerevan State University, Physical and Mathematical Sciences, 3:4450, 2010.

[129] LYSMER, J.; KUHLEMEYER, R. Finite dynamic model for infinite media. ournal of the Engineering Mechanics Division Proceedings of the American Society of Civil Engineers, EM4:859-877, 1969.

[130] COHEN, M. Silent Boundary Methods for Transient Wave Analysis. PhD dissertation, Eartquake engineering Research Laboratory, California Institute of Technology, 1981.

[131] ROSS, M. Modeling methods for silent boundaries in infinite media. Fluid-Structure Interaction, Aerospace Engineering Sciences-University of Colorado at Boulder, 5519-006(February):1-10, 2004.

[132] DROZDZ, M.; MOREAU, L.; CASTAINGS, M.; LOWE, M. J. S. ; CAWLEY, P. Efficient numerical modelling of absorbing regions for boundaries of guided waves problems. AIP Conference Proceedings, 820(1):126-133, 2006.

[133] PENG, D.; YU, T. ; YU, F. An improved 2-d ansys simulation model for saw.

[134] FATTAH, M. Y. A new mapped infinite element for dynamic analysis of soil-structure interaction problems. Diyala Journal of Engineering Sciences, p. 22-23, 2010.

[135] KOUROUSSIS, G.; VERLINDEN, O. ; CONTI, C. Finite-Dynamic Model for Infinite Media: Corrected Solution of Viscous Boundary Efficiency. Journal of Engineering Mechanics, 137(7):509-511, 2011.

[136] FRANÇOIS SEMBLAT, J.; LENTI, L. ; GANDOMZADEH, A. A simple multi-directional absorbing layer method to simulate elastic wave propagation in unbounded domains. International Journal for Numerical Methods in Engineering, 2011.

[137] NADELLA, K. S.; CESNIK, C. E. S. Numerical Simulation of Wave Propagation in Composite Plates. Proc. of SPIE, 8348, 2012.

[138] KIM, H. S. Finite Element Analysis with Paraxial \& Viscous Boundary Conditions for Elastic Wave Propagation. Engineering, 4(December):843-849, 2012. 
[139] SHEN, Y.; GIURGIUTIU, V. Effective non-reflective boundary for lamb waves: Theory, finite element implementation, and applications. Wave Motion, 58:22 - 41, 2015.

[140] WANG, C. H.; ROSE, J. T. ; CHANG, F.-K. A computerized timereversal method for structural health monitoring. In: PROCEEDINGS OF SPIE CONFERENCE ON SMART STRUCTURES AND NDE, SAN DIEGO, CA, USA, 2003.

[141] BUTENAS, G.; KAZYS, R. Investigation of time-reversal approach for detection and characterization of ultrasonic defects in dispersive plates. Ultragarsas Journal, 64(4):34-39, 2006.

[142] PARK, H. W.; SOHN, H.; LAW, K. H. ; FARRARD, C. R. Time reversal active sensing for health monitoring of a composite plate. Journal of Sound and Vibration, 302:50-66, 2007.

[143] GANGADHARAN, R.; MURTHY, C.; GOPALAKRISHNAN, S. ; BHAT, M. Time reversal technique for health monitoring of metallic structure using lamb waves. Ultrasonics, 49:696-705, 2009.

[144] PODDAR, B.; KUMAR, A.; MITRAL, M. ; MUJUMDAR, P. Time reversibility of a lamb wave for damage detection in a metallic plate. Smart Mater. Struct., 20(2), 2011.

[145] JEONG, H.; CHO, S. ; WEI, W. A baseline-free defect imaging technique in plates using time reversal of lamb waves. Chinese Phys. Lett., 28(6), 2011.

[146] ROSE, L. R. F.; WANG, C. H. Mindlin plate theory for damage detection: Source solutions. Journal of the Acoustical Society of America, 116:154-171, 2004.

[147] KERBRAT, E.; ING, R. K.; PRADA, C.; CASSEREAU, D. ; FINK, M. The D.O.R.T. method applied to detection and imaging in plates using lamb waves. In: AIP CONFERENCE PROCEEDINGS,REVIEW OF PROGRESS IN QUANTITATIVE NONDESTRUCTIVE EVALUATION: VOLUME 20., volume 557, p. 934-940, 2001.

[148] ACHENBACH, J. Reciprocity and related topics in elastodynamics. Appl. Mech. Rev, 59(1):13-32, 2006.

[149] DERODE, A.; TOURIN, A. ; FINK, M. Ultrasonic pulse compression with one-bit time reversal through multiple scattering. Journal of Applied Physics, 85(9):6343-3652, 1999. 
[150] MONTAldO, G.; ROUX, P.; DERODE, A.; NEGRERA, C. ; FINK, M. Generation of very high pressure pulses with 1-bit time reversal in a solid waveguide. Journal of Acoustic Society of America, 110(6):28492857, 2001.

[151] MONTAldO, G.; ROUX, P.; DERODE, A.; NEGRERA, C. ; FINK, M. Ultrasound shock wave generator with one-bit time reversal in a dispersive mediun, application to lithostrpisy. Applied Physics Letters, 80(5):897-899, 2002.

[152] PÉREZ, N.; MATUdA, M. Y.; NEGREIRA, C. ; ADAMOWSKI, J. C. Determination of the minimum length impulse response for time reversal focalization in acoustic cavities. AIP Conference Proceedings, 1433(1):173-176, 2012.

[153] MONTALDO, G.; PÉREZ, N.; NEGREIRA, C. ; FINK, M. The spatial focusing of a leaky time reversal chaotic cavity. Waves in Random and Complex Media, 17(1):67-83, 2007.

[154] DRAEGER, C.; AIME, J.-C. ; FINK, M. One-channel time-reversal in chaotic cavities: Experimental results. Journal of the Acoustical Society of America, 105(2):618-625, 1999.

[155] DE ROSNY, J.; TOURIN, A. ; FINK, M. Coherent backscattering of an elastic wave in a chaotic cavity. Phys. Rev. Lett., 84:1693-1695, Feb 2000.

[156] PÉREZ, N.; MELlO, P.; ADAMOWSKI; CEZAR, J. ; NEGREIRA, C. Frequency domain identification of time reversal process in acoustic cavities. In: INTERNATIONAL CONGRESS ON ULTRASONICS ICU 2009, SANTIAGO DE CHILE. PROCEEDINGS OF INTERNATIONAL CONGRESS ON ULTRASONICS ICU, 2009.

[157] LECOEUR ELECTRONIQUE. Open system - user guide for matlab interface, July 2007. Available at http://www. lecoeur-electronique.net/cariboost_files/user_27s_20guide_ 20for_20matlab_20interface_20r4.pdf. Downloaded in Dec. 2015.

[158] MATHWORKS. Mathworks r2012a documentation - matlab, 2012. Available at http://www.mathworks.com/help/techdoc/. Downloaded in Jun. 2012.

[159] FERROPERM PIEZOCERAMICS LTD., D. High quality components and materials for the electronic industry, 2003. 
[160] BOWEN, L.; GENTILMAN, R.; PHAM, H.; SERWATKA, W. ; FIORE, D. Development of 1-3 and 2-2 piezocomposite transducers. The Journal of the Acoustical Society of America, 96(5):3299-3299, 1994.

[161] SMITH, W.; AULD, B. Modeling 1-3 composite piezoelectrics: thickness-mode oscillations. Ultrasonics, Ferroelectrics, and Frequency Control, IEEE Transactions on, 38(1):40-47, Jan 1991.

[162] EISENHAURM, J. G. Regression through the origin. Teaching Statistics, 25(3), 2003.

[163] MONTGOMERY, D. C.; PECK, E. A. ; VINING, G. G. Introduction to Linear Regression Analysis. John Wiley \& Sons, 2012.

[164] LANZA DI SCALEA, F.; RIZZO, F. ; SEIBE, F. Stress measurement and defect detection in steel strands by guided stress waves. Journal of Materials in Civil Engineering, 15(3):219-227, 2003.

[165] LIN, B.; GIURGIUTIU, V. Modeling and testing of pzt and pvdf piezoelectric wafer active sensors. Smart Materials and Structures, 15(4):1085, 2006.

[166] LIN, X.; YUAN, F. G. Diagnostic lamb waves in an integrated piezoelectric sensor/actuator plate: analytical and experimental studies. Smart Materials and Structures, 10(5):907, 2001.

[167] CHAKI, S.; BOURSE, G. Guided ultrasonic waves for nondestructive monitoring of the stress levels in prestressed steel strands. Ultrasonics, 49(2):162 - 171, 2009.

[168] CHO, Y. Estimation of ultrasonic guided wave mode conversion in a plate with thickness variation. Ultrasonics, Ferroelectrics, and Frequency Control, IEEE Transactions on, 47(3):591-603, May 2000.

[169] NG, C.-T.; VEIDT, M. Scattering of the fundamental antisymmetric lamb wave at delaminations in composite laminates. The Journal of the Acoustical Society of America, 129(3):1288-1296, 2011.

[170] SOHN, H.; PARK, G.; WAIT, J. R.; LIMBACK, N. P. ; FARRAR, C. R. Wavelet-based active sensing for delamination detection in composite structures. Smart Mater. Struct., 13:153-160, 2004.

[171] HIRAO, M.; OGI, H. EMATs for science and industry: noncontacting ultrasonic measurements. Springer Netherlands, 2003. 
[172] BOSSY, E.; TALMANT, M. ; LAUGIER, P. Three-dimensional simulations of ultrasonic axial transmission velocity measurement on cortical bone models. The Journal of the Acoustical Society of America, 115(5):2314-2324, 2004.

[173] DODSON, J.; INMAN, D. Thermal sensitivity of lamb waves for structural health monitoring applications. Ultrasonics, 53(3):677 685, 2013.

[174] DODSON, J. C.; INMAN, D. J. Investigating the thermally induced acoustoelastic effect in isotropic media with lamb waves. The Journal of the Acoustical Society of America, 136(5):2532-2543, 2014.

[175] LEE, S. J.; GANDHI, N.; MICHAELS, J. E. ; MICHAELS, T. E. Comparison of the effects of applied loads and temperature variations on guided wave propagation. AIP Conference Proceedings, 1335(1):175$182,2011$.

[176] CROXFORD, A.; MOLL, J.; WILCOX, P. ; MICHAELS, J. Efficient temperature compensation strategies for guided wave structural health monitoring. Ultrasonics, 50(4-5):517 - 528, 2010. 